\title{
Design and synthesis of Fsp ${ }^{3}$-rich, bis-spirocyclic-
}

\section{based compound libraries for biological screening}

Silvia Stotani, Christoph Lorenz, Matthias Winkler, Federico Medda, Edwige Picazo, Raquel Ortega Martinez, Anna Karawajczyk, Jorge Sanchez-Quesada and Fabrizio Giordanetto*

Medicinal Chemistry, Taros Chemicals GmbH \& Co. KG, Emil-Figge-Str. 76a, 44227 Dortmund, Germany

*Corresponding Author:

Tel: +49 (0)2319742 7224; e-mail: fgiordanetto@tarosdiscovery.com

\section{Supplementary data}

\section{Contents}

General informations

Page

\author{
General informations
}

Experimental section for the bis-spirocyclic-imidazolinone library

S-3

Experimental section for the bis-spirocyclic-oxazolidine library

Stability Tests

${ }^{1} \mathrm{H},{ }^{13} \mathrm{C}$ NMR, HPLC-MS (ESI) chromatograms and mass spectra for some S-30 representative compounds from the two libraries

References 


\section{General Informations}

Chemicals and solvents were obtained from commercial suppliers and were used without further purification. All dry reactions were performed under nitrogen atmosphere using commercial dry solvents. Flash column chromatography was performed on a silica column using 230400 mesh silica gel or Grace Reveleris X2 flash chromatography system using silica gel packed Macherey Nagel Chromabond Flash BT cartridges (60,, $45 \mu \mathrm{m})$ and Grace Reveleris flash Cartridges (60 $\AA, 40 \mu \mathrm{m})$. Thin layer chromatography was performed on Macherey Nagel precoated TLC aluminum sheets with silica gel 60 UV254 (5 - $17 \mu \mathrm{m})$. TLC visualization was accomplished by irradiation with a UV lamp (254 nm) and/or staining with $\mathrm{KMnO}_{4}$ solutions. ${ }^{1} \mathrm{H}$ NMR spectra were recorded at room temperature on a Bruker Avance spectrometer operating at $300 \mathrm{MHz}$. Chemical shifts are given in ppm $(\delta)$ from tetramethylsilane as an internal standard or residual solvent peak. Significant ${ }^{1} \mathrm{H}$ NMR data are tabulated in the following order: multiplicity (s, singlet; $d$, doublet; $t$, triplet; q, quartet; $\mathrm{m}$, multiplet; dd, doublet of doublets; dt, doublet of triplets; td, triplet of doublets; br, broad), number of protons, coupling constant(s) in hertz. Proton decoupled ${ }^{13} \mathrm{C}$ NMR data were acquired at $100 \mathrm{MHz} .{ }^{13} \mathrm{C}$ chemical shifts are reported in parts per million $(\delta, \mathrm{ppm})$. All NMR data were collected at room temperature $\left(25^{\circ} \mathrm{C}\right)$. Analytical, preparative HPLC and Electron Spray Ionization condition (ESI) mass spectra were performed on an Agilent uHPLC (1290 Infinity) and an Agilent Prep-HPLC (1260 Infinity) both equipped with a Diode Array Detector and a Quadrupole MS Dusing mixture gradients of Formic acid/water/acetonitrile as system solvent. High-resolution electrospray ionization mass spectra (ESI-FTMS) were recorded on a Thermo LTQ Orbitrap (high-resolution mass spectrometer from Thermo Electron) coupled to an 'Accela' HPLC system supplied with a 'Hypersil GOLD' column (Thermo Electron). 


\section{Experimental section for the polyspiro-imidazolinone library}<smiles>N#CC1(N)CCN(Cc2ccccc2)CC1</smiles>

4-amino-1-benzylpiperidine-4-carbonitrile (2): To a stirred solution of ammonium chloride (83.4 g, $1.60 \mathrm{~mol}, 1.3 \mathrm{eq})$ in $640 \mathrm{~mL}$ of ammonium hydroxide solution $\left(25 \% \mathrm{NH}_{3}\right.$ in water, $\left.20.0 \mathrm{eq}\right)$ was added carefully sodium cyanide (73.5 g, $\left.1.50 \mathrm{~mol}, 1.25 \mathrm{eq}\right)$ and, dropwise, (over $30 \mathrm{~min}$ ) a solution of N-Benzyl-4-piperidone (230.0 g, $1.20 \mathrm{~mol}, 1.0 \mathrm{eq}$ ) in isopropyl alcohol $(230 \mathrm{~mL})$. The mixture was stirred at room temperature overnight. The solvent was removed under reduced pressure and the crude diluted with water $(\mathrm{pH}>10)$ and extracted with chloroform $(3 \times 300 \mathrm{~mL})$. The combined organic layers were washed with brine $(1 \times 300 \mathrm{~mL})$, dried over magnesium sulphate, filtered and concentrated in vacuo to yield 2 as a light yellow solid (256.0 g, 99\%). ${ }^{1} \mathrm{H}$ NMR $\left(300 \mathrm{MHz}, \mathrm{CDCl}_{3}\right) \delta 7.27-7.21(\mathrm{~m}$, $5 \mathrm{H}), 3.50(\mathrm{~s}, 2 \mathrm{H}), 2.79(\mathrm{dt}, 2 \mathrm{H}, J=3.3 \mathrm{~Hz}, J=12.2 \mathrm{~Hz}), 2.29$ (t, $2 \mathrm{H}, J=12.7 \mathrm{~Hz}), 1.94$ (d, $2 \mathrm{H}, J=13.1 \mathrm{~Hz}), 1.74(\mathrm{dd}, 3 \mathrm{H}, J=3.4 \mathrm{~Hz}, J=10.6 \mathrm{~Hz}) \mathrm{ppm}^{1}$<smiles>NC(=O)C1(N)CCN(Cc2ccccc2)CC1</smiles>

4-amino-1-benzylpiperidine-4-carboxamide (3): To a stirred solution of 2 $(78.0 \mathrm{~g}, 3.60 \mathrm{~mol})$ in dry dichloromethane $(500 \mathrm{~mL})$ was added dropwise (over 2 hours) at 0 ${ }^{\circ} \mathrm{C}$ concentrated (95 - 98\%) sulphuric acid (20.0 eq). After the addition was completed, the reaction was allowed to reach room temperature and stirred overnight. The reaction mixture was then poured slowly onto crushed ice $(3000 \mathrm{~mL})$, basified $(\mathrm{pH}=14)$ with sodium hydroxide $10 \mathrm{M}(1500 \mathrm{~mL})$ and extracted with ethyl acetate $(3 \times 1000 \mathrm{~mL})$. The combined organic layers were washed with brine $(1 \mathrm{x} 1000 \mathrm{~mL})$, dried over magnesium sulphate, filtered and concentrated in vacuo to yield 3 as a yellow solid (69.5 g, 97\%). ${ }^{1} \mathrm{H}$ NMR (300 MHz, DMSO-d $\left.d_{6}\right) \delta .39$ (br s, 1H), $7.30-7.21(\mathrm{~m}, 5 \mathrm{H}), 6.90$ (br s, 1H), $3.43(\mathrm{~s}, 2 \mathrm{H}), 2.28$ (t, $2 \mathrm{H}, J=12.5 \mathrm{~Hz}), 1.92(\mathrm{td}, 2 \mathrm{H}, J=4.2 \mathrm{~Hz}, J=12.3 \mathrm{~Hz}, J=12.6 \mathrm{~Hz}), 1.73$ (s, 2H), 1.27 (d, $2 \mathrm{H}, J=12.8 \mathrm{~Hz}) \mathrm{ppm} ;{ }^{13} \mathrm{C}\left(100 \mathrm{MHZ}, \mathrm{DMSO}-d_{6}\right) \delta 179.8,138.7,128.6,128.0,126.6,62.2$, $54.5,48.4,34.3$ ppm. $^{2}$ 
General procedure for the condensation reaction: To a stirred solution of $\mathbf{3}$ (1.0 eq) in dry methanol were added $p$-toluen sulfonic acid monohydrate $(0.2 \mathrm{eq})$ and the corresponding ketone $(1.2-3.0 \mathrm{eq})$. The mixture was heated at reflux under nitrogen for $1-6$ day(s). Once the mixture was cooled down to room temperature, the solvent was removed under reduced pressure, the residue re-dissolved in dichloromethane, washed twice with a saturated solution of sodium hydrogen carbonate and once with brine. The organic layer was dried over magnesium sulphate, filtered and concentrated in vacuo to yield the desired product. (In case of precipitate formation after methanol evaporation, the solid was filtered off, washed with a saturated solution of sodium hydrogen carbonate and dried in vacuo). See Table 1 in the paper for the structures of used ketones.

Analytical data of compounds $4\{1-7\}$ prepared according to the general condensation procedure<smiles>CC1(C)NC(=O)C2(CCN(Cc3ccccc3)CC2)N1</smiles>

8-benzyl-2,2-dimethyl-1,3,8-triazaspiro[4.5]decan-4-one $4\{1\}$ : White solid, 74\% yield. ${ }^{1} \mathrm{H}$ NMR (300 MHz, DMSO- $\left.d_{6}\right) \delta 8.20$ (br s, $\left.1 \mathrm{H}\right), 7.31-7.23(\mathrm{~m}, 5 \mathrm{H}), 3.44$ (s, 2H), $2.63(\mathrm{~d}, 2 \mathrm{H}, J=11.3 \mathrm{~Hz}), 2.53(\mathrm{~s}, 1 \mathrm{H}), 2.16(\mathrm{t}, 2 \mathrm{H}, J=11.5 \mathrm{~Hz}), 1.75(\mathrm{td}, 2 \mathrm{H}, J=4.1$ $\mathrm{Hz}, J=12.5 \mathrm{~Hz}, J=12.6 \mathrm{~Hz}), 1.37$ (d, $2 \mathrm{H}, J=12.1 \mathrm{~Hz}), 1.26(\mathrm{~s}, 6 \mathrm{H}) \mathrm{ppm} ;{ }^{13} \mathrm{C}(100 \mathrm{MHZ}$, DMSO- $\left.d_{6}\right) \delta 177.2,138.5,128.6,128.0,126.7,70.2,62.3,59.4,48.7,34.8,31.5$ ppm. HRMS (ESI-MS) calcd. for $\mathrm{C}_{16} \mathrm{H}_{23} \mathrm{~N}_{3} \mathrm{O}[\mathrm{M}+\mathrm{H}]^{+}=274.1841$. Found: 274.1835 .<smiles>O=C1NC2(CCC2)NC12CCN(Cc1ccccc1)CC2</smiles>

9-benzyl-5,9,13-triazadispiro[3.1.5 $\left.{ }^{6} \cdot 2^{4}\right]$ tridecan-12-one $4\{2\}$ : White solid, 61\%. ${ }^{1} \mathrm{H}$ NMR $\left(300 \mathrm{MHz}, \mathrm{CDCl}_{3}\right) \delta 7.26-7.19$ (m, 5H), 6.64 (br s, 1H), 3.48 (s, 2H), 2.72 
$(\mathrm{d}, 2 \mathrm{H}, J=11.1 \mathrm{~Hz}), 2.29-2.16(\mathrm{~m}, 6 \mathrm{H}), 2.01-1.91(\mathrm{~m}, 2 \mathrm{H}), 1.81-1.66(\mathrm{~m}, 4 \mathrm{H}), 1.39(\mathrm{~d}$, $2 \mathrm{H}, J=12.1 \mathrm{~Hz}$ ) ppm; HPLC-MS (ESI) $\mathrm{m} / \mathrm{z}$ calcd. For $\mathrm{C}_{17} \mathrm{H}_{23} \mathrm{~N}_{3} \mathrm{O}[\mathrm{M}+1]=286.18$. Found: 286.20 .<smiles>O=C1NC2(CCCC2)NC12CCN(Cc1ccccc1)CC2</smiles>

10-benzyl-6,10,14-triazadispiro[4.1.5 $\left.{ }^{7} .2^{5}\right]$ tetradecan-13-one $4\{3\}$ : Whitish solid, 74\% yield. ${ }^{1} \mathrm{H}$ NMR (300 MHz, $\left.\mathrm{CDCl}_{3}\right) \delta 7.95$ (br s, $\left.1 \mathrm{H}\right), 7.26-7.19(\mathrm{~m}, 5 \mathrm{H}), 3.47$ (s, 2H), $2.73(\mathrm{~d}, 2 \mathrm{H}, J=11.2 \mathrm{~Hz}), 2.01(\mathrm{t}, 2 \mathrm{H} J=14.2 \mathrm{~Hz}), 1.77-1.68(\mathrm{~m}, 8 \mathrm{H}), 1.44(\mathrm{~d}, 2 \mathrm{H}, J=$ $12.7 \mathrm{~Hz}) \mathrm{ppm} ;{ }^{13} \mathrm{C}\left(100 \mathrm{MHZ}, \mathrm{CDCl}_{3}\right) \delta 179.5,138.6,128.9,128.1,126.8,81.2,62.9,60.1$, 49.1, 41.5, 34.5, 23.1 ppm; HPLC-MS (ESI) m/z calcd. For $\mathrm{C}_{18} \mathrm{H}_{25} \mathrm{~N}_{3} \mathrm{O}[\mathrm{M}+1]=300.20$. Found: 300.20 .

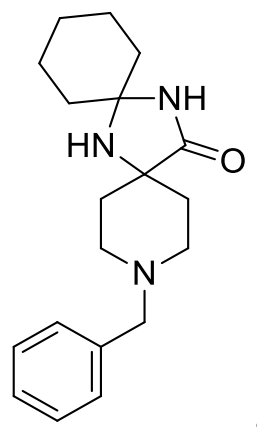

3-benzyl-3,7,14-triazadispiro[5.1.5 $\left.{ }^{8} .2^{6}\right]$ pentadecan-15-one $\quad 4\{4\}$ : Orange solid, $72 \%$ yield. ${ }^{1} \mathrm{H}$ NMR $\left(300 \mathrm{MHz}, \mathrm{CDCl}_{3}\right) \delta 7.70(\mathrm{br} \mathrm{s}, 1 \mathrm{H}), 7.26-7.17(\mathrm{~m}, 5 \mathrm{H}), 4.66(\mathrm{~s}$, 2H), $3.46(\mathrm{~s}, 2 \mathrm{H}), 2.73(\mathrm{~d}, 2 \mathrm{H}, J=11.3 \mathrm{~Hz}), 2.31(\mathrm{~s}, 1 \mathrm{H}), 2.20(\mathrm{t}, 2 \mathrm{H}, J=10.8 \mathrm{~Hz}), 2.02$ (td, $2 \mathrm{H}, J=3.6 \mathrm{~Hz}, J=12.6 \mathrm{~Hz}, J=12.8 \mathrm{~Hz}), 1.57(\mathrm{t}, 6 \mathrm{H}, J=9.9), 1.40(\mathrm{~d}, 3 \mathrm{H}, J=13.9 \mathrm{~Hz})$ ppm; ${ }^{13} \mathrm{C}\left(100 \mathrm{MHZ} \mathrm{CDCl}_{3}\right) \delta 179.2,138.6,129.0,128.1,126.9,73.0,63.1,59.6,49.2,41.0$, 35.8, 24.7, 23.0 ppm. HRMS (ESI-MS) calcd. for $\mathrm{C}_{19} \mathrm{H}_{27} \mathrm{~N}_{3} \mathrm{O}[\mathrm{M}+\mathrm{H}]^{+}=314.2154$. Found: 314.2152 . 
<smiles>O=C1NC2(CCOCC2)NC12CCN(Cc1ccccc1)CC2</smiles>

3-benzyl-11-oxa-3,7,14-triazadispiro[5.1.5 $\left.{ }^{8} 2^{6}\right]$ pentadecan-15-one $\quad 4\{5\}$ : White solid, 74\% yield. ${ }^{1} \mathrm{H}$ NMR (300 MHz, DMSO- $d_{6}$ ) $\delta 8.58$ (br s, $1 \mathrm{H}$ ), $7.39-7.28$ (m, $5 \mathrm{H}), 3.68(\mathrm{t}, 3 \mathrm{H}, J=5.1 \mathrm{~Hz}), 3.50(\mathrm{~s}, 2 \mathrm{H}), 2.72-2.66(\mathrm{~m}, 2 \mathrm{H}), 2.27(\mathrm{t}, 2 \mathrm{H}, J=10.6 \mathrm{~Hz})$, $1.80(\mathrm{td}, 2 \mathrm{H}, J=3.8 \mathrm{~Hz}, J=12.3 \mathrm{~Hz}, J=12.5 \mathrm{~Hz}), 1.63(\mathrm{~d}, 3 \mathrm{H}, J=5.4 \mathrm{~Hz}), 1.42(\mathrm{~d}, 2 \mathrm{H}, J=$ $12.5 \mathrm{~Hz}$ ) ppm. HRMS (ESI-MS) calcd. for $\mathrm{C}_{18} \mathrm{H}_{25} \mathrm{~N}_{3} \mathrm{O}_{2}[\mathrm{M}+\mathrm{H}]^{+}=316.1947$. Found: 316.1942 .<smiles>CN1CCC2(CC1)NC(=O)C1(CCN(Cc3ccccc3)CC1)N2</smiles>

3-benzyl-11-methyl-3,7,11,14-tetraazadispiro[5.1.5 ${ }^{8} .^{6}$ pentadecan-15-one 4\{6\}: White solid, 87\% yield. ${ }^{1} \mathrm{H}$ NMR (300 MHz, DMSO- $\left.d_{6}\right) \delta 8.38$ (br s, $1 \mathrm{H}$ ), $7.34-7.25$ (m, 5H), $3.45(\mathrm{~s}, 2 \mathrm{H}), 2.63(\mathrm{~d}, 2 \mathrm{H}, J=11.2 \mathrm{~Hz}), 2.45(\mathrm{~s}, 1 \mathrm{H}), 2.38-2.33(\mathrm{~m}, 4 \mathrm{H}), 2.22(\mathrm{~d}$, $1 \mathrm{H}, J=11.4 \mathrm{~Hz}), 2.15(\mathrm{~s}, 3 \mathrm{H}), 1.74(\mathrm{td}, 2 \mathrm{H}, J=3.6 \mathrm{~Hz}, J=12.3 \mathrm{~Hz}, J=12.4 \mathrm{~Hz}), 1.57$ (s, 4H), 1.35 (d, 2H, $J=12.5 \mathrm{~Hz}) \mathrm{ppm} ;{ }^{13} \mathrm{C}$ (100 MHZ, DMSO-d $) \delta$ 177.6, 138.6, 128.6, 128.0, 126.7, 70.0, 62.3, 58.7, 51.6, 48.7, 45.6, 35.0 ppm. HRMS (ESI-MS) calcd. for $\mathrm{C}_{19} \mathrm{H}_{28} \mathrm{~N}_{4} \mathrm{O}$ $[\mathrm{M}+\mathrm{H}]^{+}=329.2263$. Found: 329.2258 .<smiles>O=C1NC2(CCCCCC2)NC12CCN(Cc1ccccc1)CC2</smiles>

3-benzyl-3,7,15-triazadispiro[5.1.6 $\left.{ }^{8} .2^{6}\right]$ hexadecane-16-one $\quad 4\{7\}$ : White solid, $68 \%$ yield. ${ }^{1} \mathrm{H}$ NMR $\left(300 \mathrm{MHz}, \mathrm{CDCl}_{3}\right) \delta 7.33-7.26(\mathrm{~m}, 5 \mathrm{H}), 6.12$ (br s, $\left.1 \mathrm{H}\right), 3.53$ (s, 
2H), $2.82-2.77(\mathrm{~m}, 2 \mathrm{H}), 2.34-2.24(\mathrm{~m}, 2 \mathrm{H}), 2.12-2.03(\mathrm{~m}, 2 \mathrm{H}), 1.87-1.73(\mathrm{~m}, 5 \mathrm{H}), 1.59$ $(\mathrm{d}, 9 \mathrm{H}, J=2.6 \mathrm{~Hz})$ ppm. HRMS (ESI-MS) calcd. for $\mathrm{C}_{20} \mathrm{H}_{29} \mathrm{~N}_{3} \mathrm{O}[\mathrm{M}+\mathrm{H}]^{+}=328.2311$. Found: 328.2305.

General procedure for the acetylation reaction: To a stirred solution of the corresponding cyclized product $4\{1-7\}$ (1.0 eq) in dry dichloromethane was added $N, N$ diisopropyethylamine (DIPEA) (4.0 eq). The mixture was cooled to $0{ }^{\circ} \mathrm{C}$ and acetyl chloride (2.5 eq) was added dropwise. After the addition was completed, the mixture was allowed to warm to room temperature and then refluxed overnight. The DIPEA* $\mathrm{HCl}$ salt was filtered off and the residue was washed twice with a saturated solution of sodium hydrogen carbonate and once with brine. The organic layer was dried over magnesium sulphate, filtered and concentrated in vacuo to yield the desired acetylated product.

\section{Examples of acetylated products}<smiles>CC(=O)N1C(C)(C)NC(=O)C12CCN(Cc1ccccc1)CC2</smiles>

1-acetyl-8-benzyl-2,2-dimethyl-1,3,8-triazaspiro[4.5]decan-4-one $5\{1\}$ : Colorless oil, 67\% yield. ${ }^{1} \mathrm{H}$ NMR (300 MHz, DMSO- $\left.d_{6}\right) \delta 8.78(\mathrm{~s}, 1 \mathrm{H}), 7.37-7.30(\mathrm{~m}, 5 \mathrm{H})$, $3.54(\mathrm{~s}, 2 \mathrm{H}), 3.39$ (s, 3H), $2.84-2.76(\mathrm{~m}, 4 \mathrm{H}), 2.33-2.13(\mathrm{~m}, 4 \mathrm{H}), 1.60(\mathrm{~s}, 6 \mathrm{H}) \mathrm{ppm}$; HPLC-MS (ESI) m/z calcd. For $\mathrm{C}_{18} \mathrm{H}_{25} \mathrm{~N}_{3} \mathrm{O}_{2}[\mathrm{M}+1]=316.19$. Found: 316.25 .<smiles>CC(=O)N1C2(CCCC2)NC(=O)C12CCN(Cc1ccccc1)CC2</smiles>

6-acetyl-10-benzyl-6,10,14-triazadispiro[4.1.5.$^{7} \cdot 2^{5}$ tetradecan-13-one $5\{3\}$ : Colorless oil, 51\% yield. ${ }^{1} \mathrm{H}$ NMR $\left(300 \mathrm{MHz}, \mathrm{CDCl}_{3}\right) \delta 8.33(\mathrm{~s}, 1 \mathrm{H}), 7.25-7.19(\mathrm{~m}, 5 \mathrm{H})$, $3.50(\mathrm{~s}, 2 \mathrm{H}), 2.82-2.67(\mathrm{~m}, 4 \mathrm{H}), 2.27(\mathrm{~s}, 4 \mathrm{H}), 1.97(\mathrm{~s}, 3 \mathrm{H}), 1.86-1.75(\mathrm{~m}, 4 \mathrm{H}), 1.61(\mathrm{~s}$, 
2H), $1.43(\mathrm{~s}, 2 \mathrm{H})$ ppm. HRMS (ESI-MS) calcd. for $\mathrm{C}_{20} \mathrm{H}_{27} \mathrm{~N}_{3} \mathrm{O}_{2}[\mathrm{M}+\mathrm{H}]^{+}=342.2103$. Found: 342.2101.<smiles>CC(=O)N1C2(CCCCC2)NC(=O)C12CCN(Cc1ccccc1)CC2</smiles>

7-acetyl-3-benzyl-3,7,14-triazadispiro[5.1.5 $\left.{ }^{8} .2^{6}\right]$ pentadecan-15-one $5\{4\}$ : Whitish solid, 59\% yield. ${ }^{1} \mathrm{H}$ NMR (300 MHz, $\left.\mathrm{CDCl}_{3}\right) \delta 8.47$ (br s, $\left.1 \mathrm{H}\right), 7.33-7.24(\mathrm{~m}, 5 \mathrm{H})$, $3.55(\mathrm{~s}, 2 \mathrm{H}), 3.06-2.68(\mathrm{~m}, 6 \mathrm{H}), 2.55-2.43(\mathrm{~m}, 2 \mathrm{H}), 2.36(\mathrm{~s}, 3 \mathrm{H}), 1.86-1.66(\mathrm{~m}, 5 \mathrm{H}), 1.44$ $-1.25(\mathrm{~m}, 5 \mathrm{H})$ ppm; HPLC-MS (ESI) $\mathrm{m} / \mathrm{z}$ calcd. For $\mathrm{C}_{21} \mathrm{H}_{29} \mathrm{~N}_{3} \mathrm{O}_{2}[\mathrm{M}+1]=356.23$. Found: 356.30 .<smiles>CC(=O)N1C2(CCOCC2)NC(=O)C12CCN(Cc1ccccc1)CC2</smiles>

7-acetyl-3-benzyl-11-oxa-3,7,14-triazadispiro[5.1.5 ${ }^{8} .^{6}$ ]pentadecan-15-one 5\{5\}: Yellowish oil, 57\% yield. ${ }^{1} \mathrm{H}$ NMR (300 MHz, DMSO-d $\left.d_{6}\right) \delta 9.52$ (s, 1H), 7.32 - 7.20 $(\mathrm{m}, 5 \mathrm{H}), 3.78-3.73(\mathrm{~m}, 2 \mathrm{H}), 3.63-3.55(\mathrm{~m}, 2 \mathrm{H}), 3.50(\mathrm{~s}, 2 \mathrm{H}), 3.15-2.93(\mathrm{~m}, 2 \mathrm{H}), 2.78-$ $2.68(\mathrm{~m}, 4 \mathrm{H}), 2.24(\mathrm{~s}, 3 \mathrm{H}), 2.07(\mathrm{~s}, 2 \mathrm{H}), 1.78-1.74(\mathrm{~m}, 2 \mathrm{H}), 1.33-1.13(\mathrm{~m}, 2 \mathrm{H}) \mathrm{ppm}$; HPLC-MS (ESI) m/z calcd. For $\mathrm{C}_{20} \mathrm{H}_{27} \mathrm{~N}_{3} \mathrm{O}_{3}[\mathrm{M}+1]=358.21$. Found: 358.25 .<smiles>CC(=O)N1C2(CCN(C)CC2)NC(=O)C12CCN(Cc1ccccc1)CC2</smiles>

7-acetyl-3-benzyl-11-methyl-3,7,11,14-tetraazadispiro[5.1.5 $\left.{ }^{8} .2^{6}\right]$

pentadecan-15-one 5\{6\}: Whitish solid, 44\% yield. ${ }^{1} \mathrm{H}$ NMR (300 MHz, DMSO- $\left.d_{6}\right) \delta 9.26$ (br s, 1H), $7.32-7.25(\mathrm{~m}, 5 \mathrm{H}), 3.50(\mathrm{~s}, 2 \mathrm{H}), 3.15-2.97(\mathrm{~m}, 2 \mathrm{H}), 2.75-2.61(\mathrm{~m}, 7 \mathrm{H}), 2.31$ 
$(\mathrm{d}, 3 \mathrm{H}, J=12.0 \mathrm{~Hz}), 2.23(\mathrm{~s}, 3 \mathrm{H}), 2.17(\mathrm{~s}, 3 \mathrm{H}), 1.81-1.65(\mathrm{~m}, 2 \mathrm{H}), 1.28-1.13(\mathrm{~m}, 3 \mathrm{H})$ ppm. HRMS (ESI-MS) calcd. for $\mathrm{C}_{21} \mathrm{H}_{30} \mathrm{~N}_{4} \mathrm{O}_{2}[\mathrm{M}+\mathrm{H}]^{+}=371.2369$. Found: 371.2362.

1-benzoyl-8-benzyl-2,2-dimethyl-1,3,8-triazaspiro[4.5]decan-4-one 5\{9\} was prepared as followed:<smiles>CC1(C)NC(=O)C2(CCN(Cc3ccccc3)CC2)N1C(=O)c1ccccc1</smiles>

To a stirred solution of $\mathbf{4}\{\mathbf{1}\}$ (1.0 eq) in dry dichloromethane was added $N$, $N$ - diisopropyethylamine (DIPEA) $(6.0 \mathrm{eq})$. The mixture was cooled to $0{ }^{\circ} \mathrm{C}$ and benzoyl chloride (3.0 eq) was added dropwise. After the addition was completed, the mixture was allowed to warm to room temperature and then refluxed for three days. The DIPEA*HCl salt was filtered off and the residue was washed twice with a saturated solution of sodium hydrogen carbonate and once with brine. The organic layer was dried over magnesium sulphate, filtered and concentrated in vacuo to yield $\mathbf{5}\{9\}$ as a brown oil, 42\% yield. ${ }^{1} \mathrm{H}$ NMR $\left(300 \mathrm{MHz}, \mathrm{CDCl}_{3}\right) \delta 7.43-7.39(\mathrm{~m}, 5 \mathrm{H}), 7.35$ - $7.29(\mathrm{~m}, 5 \mathrm{H}), 4.15(\mathrm{~s}, 2 \mathrm{H}), 3.63-3.55(\mathrm{~m}$, 2H), $3.24-3.14(\mathrm{~m}, 4 \mathrm{H}), 1.82(\mathrm{~d}, 2 \mathrm{H}, J=14.3 \mathrm{~Hz}), 1.50(\mathrm{~s}, 6 \mathrm{H}) \mathrm{ppm}$. HRMS (ESI-MS) calcd. for $\mathrm{C}_{23} \mathrm{H}_{27} \mathrm{~N}_{3} \mathrm{O}_{2}[\mathrm{M}+\mathrm{H}]^{+}=378.2103$. Found: 378.2100 .

3-benzyl-7-propanoyl-3,7,14-triazadispiro[5.1.5 ${ }^{8} .^{6}$ ]pentadecan-15-one $\quad 5\{10\} \quad$ was prepared as followed:<smiles>CCC(=O)N1C2(CCCCC2)NC(=O)C12CCN(Cc1ccccc1)CC2</smiles>

To a stirred solution of $\mathbf{4}\{\mathbf{4}\}(1.0 \mathrm{eq})$ in dry dichloromethane was added $N, N$ - diisopropyethylamine (DIPEA) $(5.0 \mathrm{eq})$. The mixture was cooled to $0{ }^{\circ} \mathrm{C}$ and propionyl chloride (2.5 eq) was added dropwise. After the addition was completed, the mixture was allowed to warm to room temperature and then refluxed for two days. The DIPEA*HCl salt 
was filtered off and the residue was washed twice with a saturated solution of sodium hydrogen carbonate and once with brine. The organic layer was dried over magnesium sulphate, filtered and concentrated in vacuo to yield $\mathbf{5}\{\mathbf{1 0}\}$ as an orange oil, $18 \%$ yield. ${ }^{1} \mathrm{H}$ NMR (300 MHz, $\left.\mathrm{CDCl}_{3}\right) \delta 8.40(\mathrm{~s}, 1 \mathrm{H}), 7.35$ - $7.29(\mathrm{~m}, 5 \mathrm{H}), 3.94(\mathrm{~s}, 2 \mathrm{H}), 3.42-3.31(\mathrm{~m}$, 2H), $3.15-3.11(\mathrm{~m}, 2 \mathrm{H}), 2.95-2.69(\mathrm{~m}, 4 \mathrm{H}), 2.49(\mathrm{q}, 2 \mathrm{H}, J=7.3 \mathrm{~Hz}), 1.78-1.74(\mathrm{~m}, 4 \mathrm{H})$, $1.63-1.61(\mathrm{~m}, 1 \mathrm{H}), 1.40-1.24(\mathrm{~m}, 5 \mathrm{H}), 1.12(\mathrm{t}, 3 \mathrm{H}, J=7.3 \mathrm{~Hz})$ ppm. HRMS (ESI-MS) calcd. for $\mathrm{C}_{22} \mathrm{H}_{31} \mathrm{~N}_{3} \mathrm{O}_{2}[\mathrm{M}+\mathrm{H}]^{+}=370.2416$. Found: 370.2412 .

General procedure for the de-benzylation reaction: To a stirred solution of the N-benzyl protected compound 5\{1-10\} (1.0 eq) in dry methanol under nitrogen atmosphere, was added palladium hydroxide $20 \% \mathrm{wt}$ on carbon $(0.05 \mathrm{eq})$. The atmosphere was removed and replace by hydrogen (three times). The reaction was stirred at room temperature for $2-6$ hours. The mixture was filtered over celite and washed three times with methanol. The filtrate was concentrated till dryness to yield the desired product without further purification.

\section{Examples of de-benzylated products:}<smiles>CC(=O)N1C2(CCCC2)NC(=O)C12CCNCC2</smiles>

6-acetyl-6,10,14-triazadispiro[4.1.5 $\left.5^{7} \cdot 2^{5}\right]$ tetradecan-13-one $6\{3\}$ : White solid, 94\% yield. ${ }^{1} \mathrm{H}$ NMR (300 MHz, DMSO- $\left.d_{6}\right) \delta 9.00($ br s, $1 \mathrm{H}), 3.16(\mathrm{td}, 2 \mathrm{H}, J=2.5 \mathrm{~Hz}$, $J=12.1 \mathrm{~Hz}, J=12.2 \mathrm{~Hz}), 2.79(\mathrm{~d}, 2 \mathrm{H}, J=10.0 \mathrm{~Hz}), 2.45-2.35(\mathrm{~m}, 2 \mathrm{H}), 2.19(\mathrm{~d}, 2 \mathrm{H}, J=7.1$ Hz), 2.08 (s, 3H), 1.75 - $1.61(\mathrm{~m}, 6 \mathrm{H}), 1.48$ - 1.36 (m, 2H) ppm. HRMS (ESI-MS) calcd. for $\mathrm{C}_{13} \mathrm{H}_{21} \mathrm{~N}_{3} \mathrm{O}_{2}[\mathrm{M}+\mathrm{H}]^{+}=252.1634$. Found: 252.1631 .<smiles>CC(=O)N1C2(CCCCC2)NC(=O)C12CCNCC2</smiles>

7-acetyl-3,7,14-triazadispiro[5.1.5 $\left.{ }^{8} .2^{6}\right]$ pentadecan-15-one $6\{4\}$ : Whitish solid, 88\% yield. ${ }^{1} \mathrm{H}$ NMR (300 MHz, DMSO- $d_{6}$ ) $\delta 9.18$ (br s, $\left.1 \mathrm{H}\right), 3.32$ (s, 4H), 3.19 (td, 3H, $J=1.9 \mathrm{~Hz}, J=12.4 \mathrm{~Hz}, J=12.5 \mathrm{~Hz}), 2.81(\mathrm{dd}, 3 \mathrm{H}, J=4.7 \mathrm{~Hz}, J=11.9 \mathrm{~Hz}), 2.20(\mathrm{~s}, 3 \mathrm{H})$, 
$1.56(\mathrm{~s}, 6 \mathrm{H}), 1.33-1.22(\mathrm{~m}, 1 \mathrm{H}), 1.15-1.04(\mathrm{~m}, 1 \mathrm{H}) \mathrm{ppm}$; HPLC-MS (ESI) m/z calcd. For $\mathrm{C}_{14} \mathrm{H}_{23} \mathrm{~N}_{3} \mathrm{O}_{2}[\mathrm{M}+1]=266.18$. Found: 266.30 .<smiles>CC(=O)N1C2(CCN(C)CC2)NC(=O)C12CCNCC2</smiles>

\section{7-acetyl-11-methyl-3,7,11,14-tetraazadispiro[5.1.5 $\left.{ }^{8} 2^{6}\right]$ pentadecan-15-one}

6\{6\}: White solid, 98\% yield. ${ }^{1} \mathrm{H}$ NMR (300 MHz, DMSO- $\left.d_{6}\right) \delta 9.35$ (s, $\left.1 \mathrm{H}\right), 3.30-3.22$ (m, 2H), $3.07-3.05(\mathrm{~m}, 1 \mathrm{H}), 2.91(\mathrm{~d}, 2 \mathrm{H}, J=4.1 \mathrm{~Hz}, J=12.0 \mathrm{~Hz}), 2.61(\mathrm{~d}, 2 \mathrm{H}, J=9.0 \mathrm{~Hz}), 2.28$ $(\mathrm{t}, 3 \mathrm{H}, J=11.8 \mathrm{~Hz}), 2.18(\mathrm{~d}, 6 \mathrm{H}, J=12.9 \mathrm{~Hz}), 1.78$ (s, 3H) ppm. HRMS (ESI-MS) calcd. for $\mathrm{C}_{14} \mathrm{H}_{24} \mathrm{~N}_{4} \mathrm{O}_{2}[\mathrm{M}+\mathrm{H}]^{+}=281.1899$. Found: 281.1892.<smiles>CC1(C)NC(=O)C2(CCNCC2)N1C(=O)c1ccccc1</smiles>

1-benzoyl-2,2-dimethyl-1,3,8-triazaspiro[4.5]decan-4-one 6\{9\}: White solid, $92 \%$ yield. ${ }^{1} \mathrm{H}$ NMR (300 MHz, DMSO- $\left.d_{6}\right) \delta 7.42-7.40(\mathrm{~m}, 3 \mathrm{H}), 7.31-7.28(\mathrm{~m}, 2 \mathrm{H})$, $3.44-3.29$ (m, 2H), $3.22(\mathrm{td}, 2 \mathrm{H}, J=3.1 \mathrm{~Hz}, J=12.5 \mathrm{~Hz}, J=12.7 \mathrm{~Hz}), 2.72(\mathrm{dd}, 2 \mathrm{H}, J=4.6$ $\mathrm{Hz}, J=11.8 \mathrm{~Hz}$ ), 1.64 (d, 2H, $J=12.6 \mathrm{~Hz}), 1.39$ (s, 6H) ppm. HRMS (ESI-MS) calcd. for $\mathrm{C}_{16} \mathrm{H}_{21} \mathrm{~N}_{3} \mathrm{O}_{2}[\mathrm{M}+\mathrm{H}]^{+}=288.1634$. Found: 288.1631 . 


\section{General procedures for final diversification steps for the two libraries}

General procedure for the amide coupling (f): Reactions were performed in parallel in 15 $\mathrm{ml}$ reaction tubes in a 24 position Mettler-Toledo Miniblock ${ }^{\circledR}$ equipped with a heat transfer block and inert gas manifold. Each reaction tube was loaded with TEA (5.0 eq) and a previously prepared solution of $30 \mathrm{mg}$ of 6 (or 18) in $1 \mathrm{~mL}$ of dry THF. Then the corresponding acyl chlorides (1.5 eq) were added. The reactions were stirred at room temperature overnight. The mixtures were evaporated until dryness. The crudes were rediluted in $1.0 \mathrm{~mL}$ of $\mathrm{ACN}$, filtered and purified with preparative HPLC.

General procedure for the amide coupling (f'): Reactions were performed in parallel in 15 $\mathrm{ml}$ reaction tubes in a 24 position Mettler-Toledo Miniblock ${ }^{\circledR}$ equipped with a heat transfer block and inert gas manifold. Each reaction tube was loaded with $\mathrm{EDC} * \mathrm{HCl}(2.5 \mathrm{eq}), \mathrm{HOBt}$ (2.0 eq), DIPEA (5.0 eq) and a previously prepared solution of $30 \mathrm{mg}$ of 6 (or 18) in $1.0 \mathrm{~mL}$ of DCM. Then the corresponding acids $(2.0 \mathrm{eq})$ were added. The reactions were stirred at 50 ${ }^{\circ} \mathrm{C}$ overnight. The mixtures were evaporated until dryness. The crudes were rediluted in 1.0 $\mathrm{mL}$ of acetonitrile, filtered and purified with preparative HPLC.

General procedure for reductive amination (g): Reactions were performed in parallel in 15 $\mathrm{ml}$ reaction tubes in a 24 position Mettler-Toledo Miniblock ${ }^{\circledR}$ equipped with a heat transfer block and inert gas manifold. Each reaction tube was loaded with the corresponding aldehyde (2.0 eq), $\mathrm{NaBH}(\mathrm{OAc})_{3}(2.5$ eq.), acetic acid (2.0 eq) and $1.0 \mathrm{~mL}$ of 1,2-dichloroethene (DCE). The reaction mixture was stirred for $30 \mathrm{~min}$, then a previously prepared solution of 30 $\mathrm{mg}$ of 6 (or 18) in $1.0 \mathrm{~mL}$ DCE was added. The reactions were stirred at room temperature overnight. The mixtures were diluted with dichloromethane $(2.0 \mathrm{~mL})$ and washed with water $(1.0 \mathrm{~mL})$. The organic layers were evaporated until dryness. The crudes were rediluted in 1.0 $\mathrm{mL}$ of acetonitrile, filtered and purified with preparative HPLC.

General procedure for sulfonylation (h): Reactions were performed in parallel in $15 \mathrm{ml}$ reaction tubes in a 24 position Mettler-Toledo Miniblock ${ }^{\circledR}$ equipped with a heat transfer block and inert gas manifold. Each reaction tube was loaded with TEA (5.0 eq) and a previously prepared solution of $30 \mathrm{mg}$ of 6 (or 18) in $1 \mathrm{~mL}$ of dry THF. Then the corresponding sulfonyl chlorides $(1.5 \mathrm{eq})$ were added. The reactions were stirred at room 
temperature overnight. The mixtures were evaporated until dryness. The crudes were rediluted in $1.0 \mathrm{~mL}$ of $\mathrm{ACN}$, filtered and purified with preparative HPLC.

General procedure for urea formation (i): Reactions were performed in parallel in $15 \mathrm{ml}$ reaction tubes in a 24 position Mettler-Toledo Miniblock ${ }^{\circledR}$ equipped with a heat transfer block and inert gas manifold. Each reaction tube was loaded with TEA (5.0 eq) and a previously prepared solution of $30 \mathrm{mg}$ of 6 (or 18) in $1 \mathrm{~mL}$ of DCM. Then the corresponding isocyanates (1.5 eq) were added. The reactions were stirred at room temperature overnight. The mixtures were evaporated until dryness. The crudes were rediluted in $1.0 \mathrm{~mL}$ of ACN, filtered and purified with preparative HPLC.

\section{Examples of final diversification products (bis-spiro-imidazolinone library)}

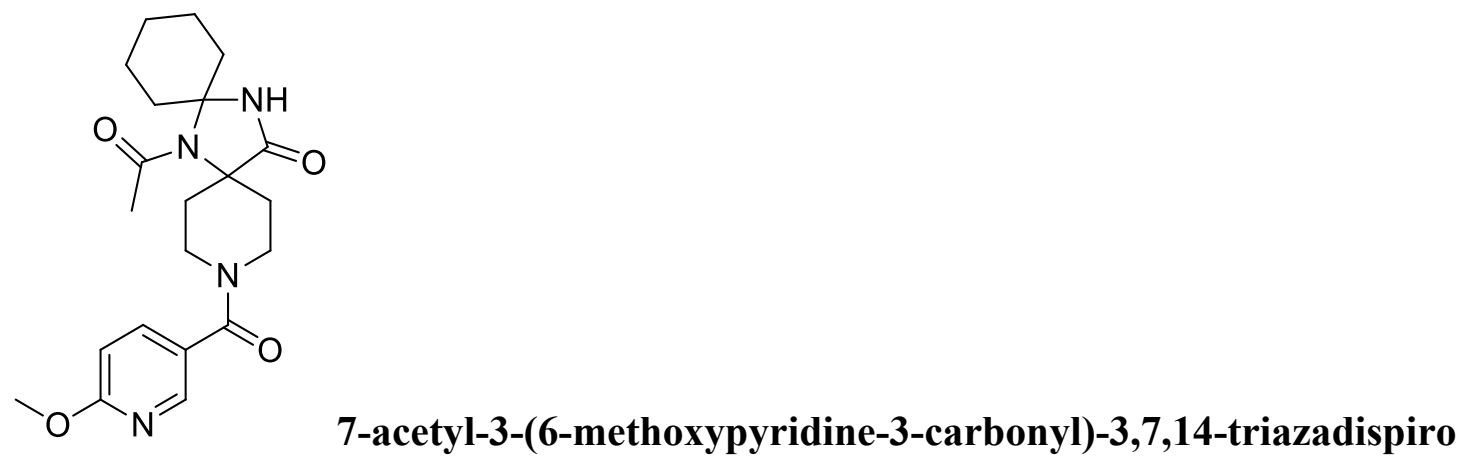

[5.1.5.$^{8}{ }^{6}$ ]pentadecan-15-one 7\{4.1\}: White solid. ${ }^{1} \mathrm{H}$ NMR $\left(300 \mathrm{MHz}, \mathrm{CDCl}_{3}\right) \delta 8.22(\mathrm{~s}$, 1H), $7.64(\mathrm{~d}, 1 \mathrm{H}, J=8.4 \mathrm{~Hz}), 6.71(\mathrm{~d}, 1 \mathrm{H}, J=8.3 \mathrm{~Hz}), 4.54-4.45(\mathrm{~m}, 2 \mathrm{H}), 3.89(\mathrm{~s}, 3 \mathrm{H})$, $3.76-3.65(\mathrm{~m}, 2 \mathrm{H}), 3.16-2.86(\mathrm{~m}, 4 \mathrm{H}), 2.20(\mathrm{~s}, 3 \mathrm{H}), 1.77(\mathrm{~s}, 7 \mathrm{H}), 1.49-1.41(\mathrm{~m}, 3 \mathrm{H})$ ppm; HRMS (ESI-MS) calcd. for $\mathrm{C}_{21} \mathrm{H}_{28} \mathrm{~N}_{4} \mathrm{O}_{4}[\mathrm{M}+\mathrm{H}]^{+}=401.2111$. Found: 401.2108.<smiles>CCC(C)(C)C(=O)N1CCC2(CC1)C(=O)NC1(CCOCC1)N2C(C)=O</smiles>

7-acetyl-3-(2,2-dimethylbutanoyl)-11-oxa-3,7,14-triazadispiro[5.1.5 $\left.{ }^{8} .2^{6}\right]$

pentadecan-15-one 7\{5.1\}: Colorless oil. ${ }^{1} \mathrm{H}$ NMR (300 MHz, $\left.\mathrm{CDCl}_{3}\right) \delta 4.42(\mathrm{~s}, 2 \mathrm{H}), 4.04$ (d, 2H, $J=6.8 \mathrm{~Hz}), 3.62(\mathrm{~s}, 4 \mathrm{H}), 3.43-3.28(\mathrm{~m}, 2 \mathrm{H}), 2.48-2.34(\mathrm{~m}, 2 \mathrm{H}), 2.23(\mathrm{~s}, 3 \mathrm{H}), 1.92$ $-1.81(\mathrm{~m}, 2 \mathrm{H}), 1.65(\mathrm{dd}, 2 \mathrm{H}, J=6.6 \mathrm{~Hz}, J=13.3 \mathrm{~Hz}), 1.52-1.36(\mathrm{~m}, 2 \mathrm{H}), 1.26(\mathrm{~m}, 6 \mathrm{H})$, 
$0.92(\mathrm{t}, 3 \mathrm{H}, J=6.9 \mathrm{~Hz})$ ppm; HPLC-MS (ESI) m/z calcd. For $\mathrm{C}_{19} \mathrm{H}_{31} \mathrm{~N}_{3} \mathrm{O}_{4}[\mathrm{M}+1]=366.23$. Found: 366.40 .<smiles>CCN1CCC2(CC1)C(=O)NC1(CCCC1)N2C(C)=O</smiles>

6-acetyl-10-ethyl-6,10,14-triazadispiro[4.1.5 $\left.5^{7} \cdot 2^{5}\right]$ tetradecan-13-one $8\{3.1\}$ :

White solid. ${ }^{1} \mathrm{H}$ NMR $\left(300 \mathrm{MHz}, \mathrm{CDCl}_{3}\right) \delta 8.48(\mathrm{~s}, 1 \mathrm{H}), 3.60(\mathrm{t}, 2 \mathrm{H}, J=11.1 \mathrm{~Hz}), 3.47-$ $3.44(\mathrm{~m}, 2 \mathrm{H}), 2.92(\mathrm{~s}, 2 \mathrm{H}), 2.65(\mathrm{~s}, 2 \mathrm{H}), 2.29(\mathrm{~s}, 3 \mathrm{H}), 1.97(\mathrm{~s}, 3 \mathrm{H}), 1.71-1.64(\mathrm{~m}, 5 \mathrm{H}), 1.35$ (dd, $2 \mathrm{H}, J=7.2 \mathrm{~Hz}, J=14.5 \mathrm{~Hz}$ ), 0.93 (t, 3H, $J=6.8 \mathrm{~Hz}$ ) ppm; HPLC-MS (ESI) m/z calcd. For $\mathrm{C}_{15} \mathrm{H}_{25} \mathrm{~N}_{3} \mathrm{O}_{2}[\mathrm{M}+1]=280.19$. Found: 280.20 .<smiles>CC(=O)N1C2(CCCC2)NC(=O)C12CCN(CCC(C)(C)C)CC2</smiles>

6-acetyl-10-(3,3-dimethylbutyl)-6,10,14-triazadispiro[4.1.5.$\left.^{7} \cdot 2^{5}\right]$ tetradecan-

13-one 8\{3.2\}: White solid. ${ }^{1} \mathrm{H}$ NMR $\left(300 \mathrm{MHz}, \mathrm{CDCl}_{3}\right) \delta 8.51(\mathrm{~d}, 1 \mathrm{H}, J=3.0 \mathrm{~Hz}), 3.55$ (t, $2 \mathrm{H}, J=9.7 \mathrm{~Hz}), 3.40(\mathrm{~s}, 2 \mathrm{H}), 2.90(\mathrm{~s}, 4 \mathrm{H}), 2.65(\mathrm{~s}, 2 \mathrm{H}), 2.29(3 \mathrm{H}), 1.96(\mathrm{~s}, 3 \mathrm{H}), 1.60-1.55$ (m, 7H), 0.92 (s, 9H) ppm; HRMS (ESI-MS) calcd. for $\mathrm{C}_{19} \mathrm{H}_{33} \mathrm{~N}_{3} \mathrm{O}_{2}[\mathrm{M}+\mathrm{H}]^{+}=336.2573$. Found: 336.2571 .<smiles>CCOc1ccc(CN2CCC3(CC2)C(=O)NC2(CCCC2)N3C(C)=O)cc1OC</smiles>

triazadispiro[4.1.5 $.^{7} .^{5}$ tetradecan-13-one 8\{3.3\}: White solid. ${ }^{1} \mathrm{H} \mathrm{NMR}\left(300 \mathrm{MHz}, \mathrm{CDCl}_{3}\right)$ 
$\delta 8.50(\mathrm{~s}, 1 \mathrm{H}), 7.04(\mathrm{~s}, 1 \mathrm{H}), 6.86-6.78(\mathrm{~m}, 1 \mathrm{H}), 5.53(\mathrm{~s}, 2 \mathrm{H}), 4.07(\mathrm{dd}, 2 \mathrm{H}, J=6.8 \mathrm{~Hz}, J=$ $13.7 \mathrm{~Hz}), 3.84(\mathrm{~s}, 3 \mathrm{H}), 3.51-3.45(\mathrm{~m}, 4 \mathrm{H}), 3.31(\mathrm{~s}, 4 \mathrm{H}), 2.78-2.61(\mathrm{~m}, 2 \mathrm{H}), 2.28(\mathrm{~s}, 2 \mathrm{H})$, $1.92(\mathrm{~s}, 3 \mathrm{H}), 1.62(\mathrm{~s}, 2 \mathrm{H}), 1.44(\mathrm{t}, 3 \mathrm{H}, J=6.7 \mathrm{~Hz}) \mathrm{ppm}$; HRMS (ESI-MS) calcd. for $\mathrm{C}_{23} \mathrm{H}_{33} \mathrm{~N}_{3} \mathrm{O}_{4}[\mathrm{M}+\mathrm{H}]^{+}=416.2471$. Found: 416.2468 .

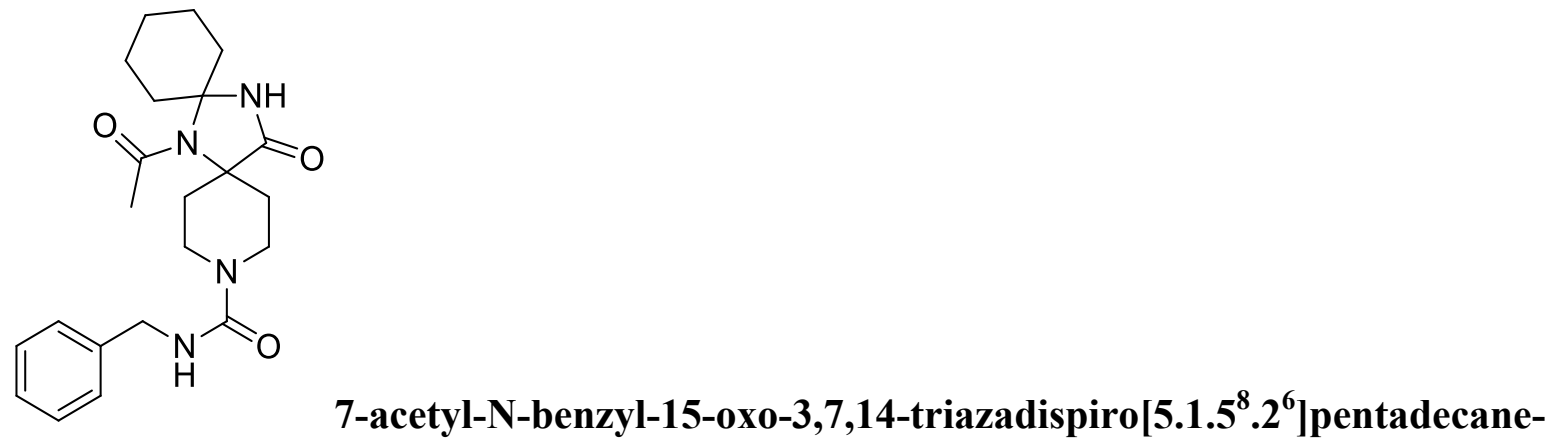

3-carboxamide 10\{4.1\}: White solid. ${ }^{1} \mathrm{H}$ NMR (300 MHz, $\left.\mathrm{CDCl}_{3}\right) \delta 7.31-7.27(\mathrm{~m}, 5 \mathrm{H})$, $4.44-4.37$ (m, 4H), $3.94-3.80(\mathrm{~m}, 2 \mathrm{H}), 3.70-3.63(\mathrm{~m}, 4 \mathrm{H}), 2.16(\mathrm{~s}, 3 \mathrm{H}), 1.89-1.76(\mathrm{~m}$, $7 \mathrm{H}), 1.46(\mathrm{~m}, 3 \mathrm{H})$ ppm. HRMS (ESI-MS) calcd. for $\mathrm{C}_{22} \mathrm{H}_{30} \mathrm{~N}_{4} \mathrm{O}_{3}[\mathrm{M}+\mathrm{H}]^{+}=399.2318$. Found: 399.2312 .<smiles>CC(=O)N1C2(CCOCC2)NC(=O)C12CCN(C(=O)Nc1cccc(C(F)(F)F)c1)CC2</smiles>

7-acetyl-15-oxo-N-[3-(trifluoromethyl)phenyl]-11-oxa-3,7,14-

triazadispiro[5.1.5 ${ }^{8} 2^{6}$ ]pentadecane-3-carboxamide $10\{5.1\}: 7.65(\mathrm{~s}, 1 \mathrm{H}), 7.58(\mathrm{~d}, 1 \mathrm{H}, J=$ $7.5 \mathrm{~Hz}), 7.43-7.38(\mathrm{~m}, 1 \mathrm{H}), 7.27(\mathrm{~s}, 1 \mathrm{H}), 4.09-4.00(\mathrm{~m}, 4 \mathrm{H}), 3.79$ (t, 2H, $J=11.3 \mathrm{~Hz}), 3.61$ - $3.53(\mathrm{~m}, 2 \mathrm{H}), 2.28(\mathrm{~s}, 3 \mathrm{H}), 1.96-1.81(\mathrm{~m}, 2 \mathrm{H}), 1.59$ (s, 6H) ppm. HRMS (ESI-MS) calcd. for $\mathrm{C}_{21} \mathrm{H}_{25} \mathrm{~F}_{3} \mathrm{~N}_{4} \mathrm{O}_{4}[\mathrm{M}+\mathrm{H}]^{+}=455.1828$. Found: 455.1822 . 


\section{Experimental section for the polyspiro-oxazolidine library}

For the conditions of the cyanation step (a) see above

\section{Examples of cyanation products}<smiles>N#CC1(N)CCCC1</smiles>

1-aminocyclopentanecarbonitrile 12\{3\}: Brown oil, 97\% yield. ${ }^{1} \mathrm{H}$ NMR (300 $\left.\mathrm{MHz}, \mathrm{CDCl}_{3}\right) \delta 2.16-2.06(\mathrm{~m}, 2 \mathrm{H}), 1.91-1.77(\mathrm{~m}, 6 \mathrm{H}), 1.70(\mathrm{br} \mathrm{s}, 2 \mathrm{H}) \mathrm{ppm}^{3}$<smiles>N#CC1(N)CCOCC1</smiles>

4-aminooxane-4-carbonitrile 12\{5\}: Yellow oil, 88\% yield. ${ }^{1} \mathrm{H}$ NMR $(300 \mathrm{MHz}$, $\left.\mathrm{CDCl}_{3}\right) \delta 3.97(\mathrm{dt}, 2 \mathrm{H}, J=4.1 \mathrm{~Hz}, J=12.3 \mathrm{~Hz}), 3.64(\mathrm{td}, 2 \mathrm{H}, J=2.4 \mathrm{~Hz}, J=12.3 \mathrm{~Hz}, J=$ $12.6 \mathrm{~Hz}), 1.98(\mathrm{dd}, 2 \mathrm{H}, J=1.7 \mathrm{~Hz}, J=13.4 \mathrm{~Hz}), 1.83($ br s, $2 \mathrm{H}), 1.74(\mathrm{td}, 2 \mathrm{H}, J=4.1 \mathrm{~Hz}, J$ $=10.0 \mathrm{~Hz}, J=10.2 \mathrm{~Hz}) \mathrm{ppm}^{4}$<smiles>CN1CCC(N)(C#N)CC1</smiles>

4-amino-1-methylpiperidine-4-carbonitrile 12\{6\}: Yellow oil, 81\% yield. ${ }^{1} \mathrm{H}$ NMR (300 MHz, CDCl3) $\delta 2.78(\mathrm{~d}, 2 \mathrm{H}, \mathrm{J}=12.4 \mathrm{~Hz}), 2.31$ (s, 3H), $2.00(\mathrm{dd}, 2 \mathrm{H}, \mathrm{J}=2.0 \mathrm{~Hz}$, $\mathrm{J}=11.1 \mathrm{~Hz}), 1.81-1.72(\mathrm{~m}, 4 \mathrm{H}) \mathrm{ppm}^{5}$

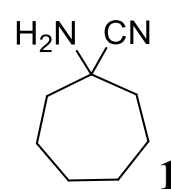

1-aminocycloheptanecarbonitrile 12\{7\}: Yellow oil, 92\% yield. ${ }^{1} \mathrm{H}$ NMR (300 $\mathrm{MHz}, \mathrm{CDCl} 3) \delta 3.46(\mathrm{~s}, 2 \mathrm{H}), 2.72(\mathrm{dd}, 2 \mathrm{H}, \mathrm{J}=3.6 \mathrm{~Hz}, \mathrm{~J}=7.8 \mathrm{~Hz}), 2.22(\mathrm{t}, 2 \mathrm{H}, \mathrm{J}=10.6 \mathrm{~Hz})$, $2.00(\mathrm{td}, 2 \mathrm{H}, \mathrm{J}=3.9 \mathrm{~Hz}, \mathrm{~J}=12.5 \mathrm{~Hz}, \mathrm{~J}=12.8 \mathrm{~Hz}), 1.70(\mathrm{~d}, 4 \mathrm{H}, \mathrm{J}=7.3 \mathrm{~Hz}), 1.43$ (d, 2H, J = $12.8 \mathrm{~Hz}) \mathrm{ppm}^{6}$ 
General procedure for the acidic hydrolysis: To a stirred solution of the cyano compound 12\{2-3, 5-7\} (1.0 eq) in 1,4-dioxane, concentrated hydrochloric acid (37\%, 20 eq) was added dropwise. The mixture was stirred at reflux for 8 hours. The solvent was removed under reduced pressure and the residue re-dissolved in methyl-tert-butylether/acetonitrile (1:3) and stirred at room temperature for 15 minutes. The mixture was filtered and the solid dried in vacuo to yield the desired products $13\{2-3,5-7\}$.

\section{Examples of hydrolysis products}

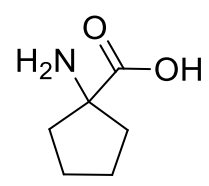

1-aminocyclopentane-1-carboxylic acid 13\{3\}: Whitish solid, 99\% yield. ${ }^{1} \mathrm{H}$ NMR (300 MHz, D $2 \mathrm{O}) \delta 2.27-2.18(\mathrm{~m}, 2 \mathrm{H}), 1.89-1.76(\mathrm{~m}, 6 \mathrm{H}) \mathrm{ppm}^{7}$<smiles>NC1(C(=O)O)CCOCC1</smiles>

4-aminooxane-4-carboxylic acid $13\{5\}$ : White solid, 99\% yield. ${ }^{1} \mathrm{H}$ NMR (300 $\left.\mathrm{MHz}, \mathrm{D}_{2} \mathrm{O}\right) \delta 3.88(\mathrm{t}, 4 \mathrm{H}, J=5.4 \mathrm{~Hz}), 2.25(\mathrm{dt}, 2 \mathrm{H}, J=3.8 \mathrm{~Hz}, J=14.1 \mathrm{~Hz}), 1.95-1.86(\mathrm{~m}$, 2H) $\mathrm{ppm}^{8}$

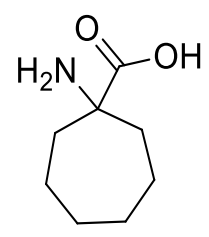

1-aminocycloheptane-1-carboxylic acid 13\{7\}: White solid, 99\% yield. ${ }^{1} \mathrm{H}$ NMR (300 MHz, D $2 \mathrm{O}) \delta 2.34-2.27(\mathrm{~m}, 2 \mathrm{H}), 1.92(\mathrm{dd}, 2 \mathrm{H}, J=8.7 \mathrm{~Hz}, J=15.1 \mathrm{~Hz}), 1.81-$ 1.74 (m, 2H), 1.64 (s, 6H) ppm. ${ }^{7}$ 
General procedure for the methyl ester protection: To a stirred solution of the $\alpha$ aminoacid compound 13\{2-3, 5-7\} (and the commercially available 1-aminocyclohexane-1carboxylic acid) (1.0 eq) in $\mathrm{MeOH}$, was added dropwise at $0{ }^{\circ} \mathrm{C}$ thionyl chloride (6.0 eq). The mixture was allowed to reach room temperature and stirred overnight. The solvent was removed under reduced pressure, the residue re-dissolved in isopropyl alcohol/chloroform (3:7) and extracted twice with a saturated solution of sodium hydrogen carbonate $(\mathrm{pH} \sim 9)$ and once with brine. The combined organic layers were dried over magnesium sulphate, filtered and concentrated in vacuo to yield the desired products $14\{2-7\}$

\section{Examples of methyl ester protected products}

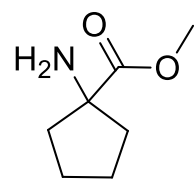

methyl 1-aminocyclopentane-1-carboxylate 14\{3\}: Orange oil, $67 \%$ yield. ${ }^{1} \mathrm{H}$ NMR $\left(300 \mathrm{MHz}, \mathrm{D}_{2} \mathrm{O}\right) \delta 3.70(\mathrm{~s}, 3 \mathrm{H}), 2.19-2.12(\mathrm{~m}, 2 \mathrm{H}), 1.83-1.71(\mathrm{~m}, 6 \mathrm{H}) \mathrm{ppm} ;{ }^{13} \mathrm{C}(100$ MHZ, $\left.\mathrm{D}_{2} \mathrm{O}\right) \delta 174.3,65.0,53.5,36.6,25.0 \mathrm{ppm}$; HPLC-MS (ESI) $\mathrm{m} / \mathrm{z}$ calcd. For $\mathrm{C}_{7} \mathrm{H}_{13} \mathrm{NO}_{2}$ $[\mathrm{M}+1]=144.09$. Found: $144.20 .^{7}$<smiles>COC(=O)C1(N)CCCCC1</smiles>

methyl 1-aminocyclohexane-1-carboxylate 14\{4\}: Colorless oil, $82 \%$ yield. ${ }^{1} \mathrm{H}$ NMR (300 MHz, $\left.\mathrm{CDCl}_{3}\right) \delta 3.65(\mathrm{~s}, 3 \mathrm{H}), 1.86(\mathrm{t}, 2 \mathrm{H}, J=9.5 \mathrm{~Hz}), 1.63-1.55(\mathrm{~m}, 4 \mathrm{H}), 1.40$ $(\mathrm{d}, 6 \mathrm{H}, J=8.9 \mathrm{~Hz}) \mathrm{ppm}$; HPLC-MS (ESI) m/z calcd. For $\mathrm{C}_{8} \mathrm{H}_{15} \mathrm{NO}_{2}[\mathrm{M}+1]=158.11$. Found: $158.30 .^{9}$<smiles>COC(=O)C1(N)CCOCC1</smiles>

methyl-4-aminooxane-4-carboxylate 14\{5\}: Colorless oil, 45\% yield. ${ }^{1} \mathrm{H}$ NMR (300 MHz, $\left.\mathrm{D}_{2} \mathrm{O}\right) \delta 3.89-3.82(\mathrm{~m}, 2 \mathrm{H}), 3.77(\mathrm{~s}, 3 \mathrm{H}), 3.72-3.64(\mathrm{~m}, 2 \mathrm{H}), 2.16-2.08(\mathrm{~m}$, 2H), $1.65-1.58(\mathrm{~m}, 2 \mathrm{H}) \mathrm{ppm} ;{ }^{13} \mathrm{C}\left(100 \mathrm{MHZ}, \mathrm{D}_{2} \mathrm{O}\right) \delta 177.6,63.6,54.6,52.9,34.1 \mathrm{ppm}$; HPLC-MS (ESI) $\mathrm{m} / \mathrm{z}$ calcd. For $\mathrm{C}_{7} \mathrm{H}_{13} \mathrm{NO}_{3}[\mathrm{M}-1]=158.09$. Found: $158.30{ }^{8}$ 
General procedure for the reduction reaction: To a stirred solution of Red-Al sodium bis(2-methoxyethoxy)aluminum hydride solution ( $70 \%$ in toluene, 3.0 eq) cooled to $0{ }^{\circ} \mathrm{C}$ was added dropwise a solution of the $\alpha$-aminoesther $14\{2-7\}$ in toluene (1.0 eq). The mixture was allowed to reach room temperature and then heated at reflux for 2 hours. Once the mixture was cooled to room temperature, it was carefully poured onto a cold mixture of water/ethanol (1:1). The precipitate was filtered off and the mother liquor was extracted 5 times with a mixture of isopropyl alcohol/chloroform (3:7). The combined organic layers were dried over magnesium sulphate, filtered and concentrated in vacuo to yield the desired products $15\{2-7\}$

\section{Examples of reduction products}

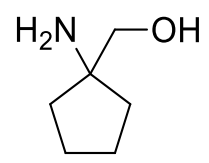

(1-aminocyclopentyl)methanol 15\{3\}: Whitish solid, 74\% yield. ${ }^{1} \mathrm{H}$ NMR (300 $\left.\mathrm{MHz}, \mathrm{CDCl}_{3}\right) \delta 3.68-3.65(\mathrm{~m}, 1 \mathrm{H}), 3.46-3.45(\mathrm{~m}, 1 \mathrm{H}), 3.35(\mathrm{~d}, 2 \mathrm{H}, J=2.4 \mathrm{~Hz}), 2.27(\mathrm{~s}$, $1 \mathrm{H}), 2.19-2.12(\mathrm{~m}, 2 \mathrm{H}), 1.87-1.76(\mathrm{~m}, 1 \mathrm{H}) \mathrm{ppm}^{10}$<smiles>NC1(CO)CCCCC1</smiles>

(1-aminocyclohexyl)methanol 15\{4\}: White solid, 99\% yield. ${ }^{1} \mathrm{H}$ NMR (300 $\left.\mathrm{MHz}, \mathrm{CDCl}_{3}\right) \delta 3.73-3.70(\mathrm{~m}, 1 \mathrm{H}), 3.51-3.48(\mathrm{~m}, 1 \mathrm{H}), 3.39(\mathrm{~s}, 1 \mathrm{H}), 3.32(\mathrm{~s}, 2 \mathrm{H}), 1.99$ (s, $2 \mathrm{H}), 1.47(\mathrm{~d}, 4 \mathrm{H}, J=4.0 \mathrm{~Hz}), 1.42-1.36(\mathrm{~m}, 4 \mathrm{H}) \mathrm{ppm}^{11}$<smiles>NC1(CO)CCOCC1</smiles>

(4-aminooxan-4-yl)methanol 15\{5\}: Orange oil, 76\% yield. ${ }^{1} \mathrm{H}$ NMR (300 $\left.\mathrm{MHz}, \mathrm{CDCl}_{3}\right) \delta 3.68-3.64(\mathrm{~m}, 4 \mathrm{H}), 3.33(\mathrm{~s}, 2 \mathrm{H}), 2.07$ (br s, 3H), $1.65-1.56(\mathrm{~m}, 2 \mathrm{H}), 1.35$ $\left(\mathrm{dt}, 2 \mathrm{H}, J=3.6 \mathrm{~Hz}, J=12.5 \mathrm{~Hz}\right.$ ) ppm. HPLC-MS (ESI) m/z calcd. For $\mathrm{C}_{6} \mathrm{H}_{13} \mathrm{NO}_{2}[\mathrm{M}+1]=$ 132.09. Found: $132.20 .^{10}$ 
General procedure for the condensation reaction: To a stirred solution of the amino alcohol 15\{2-7\} (and the commercially available 2-amino-2-methylpropan-1-ol) (1.0 eq) in dry methanol were added $p$-toluen sulfonic acid monohydrate $(0.2$ eq $)$ and $N$ benzylpiperidone $(1.1 \mathrm{eq})$. The mixture was heated at reflux under nitrogen for 4 hours. Once the mixture was cooled down to room temperature, the solvent was removed under reduced pressure, the residue re-dissolved in ethylacetate, extracted twice with a saturated solution of sodium hydrogen carbonate and once with brine. The combined organic layers were dried over magnesium sulphate, filtered and concentrated in vacuo to yield the desired products $16\{1-7\}$.

\section{Examples of condensation products}<smiles>CC1(C)COC2(CCN(Cc3ccccc3)CC2)N1</smiles>

8-benzyl-3,3-dimethyl-1-oxa-4,8-diazaspiro[4.5]decane 16\{1\}: White solid, $67 \%$ yield. ${ }^{1} \mathrm{H}$ NMR $\left(300 \mathrm{MHz}, \mathrm{CDCl}_{3}\right) \delta 7.26(\mathrm{~m}, 5 \mathrm{H}), 3.51(\mathrm{~s}, 2 \mathrm{H}), 3.46(\mathrm{~s}, 2 \mathrm{H}), 2.53-$ 2.49 (m, 2H), $2.44-2.40(\mathrm{~m}, 2 \mathrm{H}), 1.71(\mathrm{~m}, 4 \mathrm{H}), 1.18$ (s, 6H) ppm; HPLC-MS (ESI) m/z calcd. For $\mathrm{C}_{16} \mathrm{H}_{24} \mathrm{~N}_{2} \mathrm{O}[\mathrm{M}+1]=261.19$. Found: 261.30 .<smiles>c1ccc(CN2CCC3(CC2)NC2(CCCC2)CO3)cc1</smiles>

10-benzyl-13-oxa-6,10-diazaspiro $\left[4.1 .5^{7} \cdot 2^{5}\right]$ tetradecane $16\{3\}$ : Orange oil, $61 \%$ yield. ${ }^{1} \mathrm{H}$ NMR $\left(300 \mathrm{MHz}, \mathrm{CDCl}_{3}\right) \delta 7.32-7.24(\mathrm{~m}, 5 \mathrm{H}), 3.62(\mathrm{~s}, 2 \mathrm{H}), 3.54(\mathrm{~s}, 2 \mathrm{H})$, $2.60-2.57(\mathrm{~m}, 2 \mathrm{H}), 2.51-2.43(\mathrm{~m}, 2 \mathrm{H}), 1.78-1.68(\mathrm{~m}, 10 \mathrm{H}), 1.59-1.53(\mathrm{~m}, 2 \mathrm{H}) \mathrm{ppm}$. HRMS (ESI-MS) calcd. for $\mathrm{C}_{18} \mathrm{H}_{26} \mathrm{~N}_{2} \mathrm{O}[\mathrm{M}+\mathrm{H}]^{+}=287.2045$. Found: 287.2037. 
3-benzyl-15-oxa-3,7-diazadispiro[5.1.5 $\left.{ }^{8} 2^{6}\right]$ pentadecane $16\{4\}$ : Colorless oil, 38\% yield. ${ }^{1} \mathrm{H}$ NMR (300 MHz, $\left.\mathrm{CDCl}_{3}\right) \delta 7.27-7.14(\mathrm{~m}, 5 \mathrm{H}), 3.56(\mathrm{~s}, 2 \mathrm{H}), 3.45(\mathrm{~s}, 2 \mathrm{H})$, $2.54-2.48(\mathrm{~m}, 2 \mathrm{H}), 2.42-2.34(\mathrm{~m}, 2 \mathrm{H}), 1.67(\mathrm{t}, 4 \mathrm{H}, J=5.6 \mathrm{~Hz}), 1.58-1.38(\mathrm{~m}, 7 \mathrm{H}), 1.29-$ 1.19 (m, 3H) ppm; HPLC-MS (ESI) m/z calcd. For $\mathrm{C}_{19} \mathrm{H}_{28} \mathrm{~N}_{2} \mathrm{O}[\mathrm{M}+1]=301.22$. Found: 301.30 .<smiles>c1ccc(CN2CCC3(CC2)NC2(CCOCC2)CO3)cc1</smiles>

11-benzyl-3,14-dioxa-7,11-diazadispiro[5.1.5 $\left.{ }^{8} .2^{6}\right]$ pentadecane $16\{5\}$ : Orange oil, 51\% yield. ${ }^{1} \mathrm{H}$ NMR (300 MHz, $\left.\mathrm{CDCl}_{3}\right) \delta 7.25$ - $7.18(\mathrm{~m}, 5 \mathrm{H}), 3.80-3.73(\mathrm{~m}, 2 \mathrm{H}), 3.62$ $(\mathrm{s}, 2 \mathrm{H}), 3.52-3.46(\mathrm{~m}, 4 \mathrm{H}), 2.52-2.48(\mathrm{~m}, 2 \mathrm{H}), 2.43-2.35(\mathrm{~m}, 2 \mathrm{H}), 1.67(\mathrm{t}, 4 \mathrm{H}, J=5.5$ $\mathrm{Hz}$ ), $1.61(\mathrm{t}, 4 \mathrm{H}, J=5.4 \mathrm{~Hz}) \mathrm{ppm}$; HRMS (ESI-MS) calcd. for $\mathrm{C}_{18} \mathrm{H}_{26} \mathrm{~N}_{2} \mathrm{O}_{2}[\mathrm{M}+\mathrm{H}]^{+}=$ 303.1994. Found: 303.1998.

For the acetylation conditions (step d) see above.

\section{Examples of acylated products}<smiles>CC(=O)N1C(C)(C)COC12CCN(Cc1ccccc1)CC2</smiles> 
2H), $3.47(\mathrm{~s}, 2 \mathrm{H}), 2.91(\mathrm{td}, 2 \mathrm{H}, J=4.6 \mathrm{~Hz}, J=13.1 \mathrm{~Hz}, J=13.2 \mathrm{~Hz}), 2.70-2.66(\mathrm{~m}, 2 \mathrm{H})$, $2.25(\mathrm{t}, 4 \mathrm{H}, J=11.1 \mathrm{~Hz}), 2.07(\mathrm{~s}, 3 \mathrm{H}), 1.37$ (d, 6H, $J=10.1 \mathrm{~Hz})$ ppm; HRMS (ESI-MS) calcd. for $\mathrm{C}_{18} \mathrm{H}_{26} \mathrm{~N}_{2} \mathrm{O}_{2}[\mathrm{M}+\mathrm{H}]^{+}=303.1994$. Found: 303.1991 .<smiles>CC(=O)N1C2(CCCC2)COC12CCN(Cc1ccccc1)CC2</smiles>

1-\{10-benzyl-13-oxa-6,10-diazadispiro[4.1.5.$\left.^{7} \cdot 2^{5}\right]$ tetradecan-6-yl\} ethan-1-one 17\{3\}: Orange oil, 96\% yield. ${ }^{1} \mathrm{H}$ NMR $\left(300 \mathrm{MHz} \mathrm{CDCl}_{3}\right) \delta 7.25-7.20(\mathrm{~m}, 5 \mathrm{H}), 3.59(\mathrm{~d}$, $2 \mathrm{H}, J=16.0 \mathrm{~Hz}), 3.46(\mathrm{~s}, 2 \mathrm{H}), 2.89$ (t, 2H, $J=12.6 \mathrm{~Hz}), 2.70(\mathrm{~d}, 2 \mathrm{H}, J=9.9 \mathrm{~Hz}), 2.12$ (s, 3H), $1.74-1.61(\mathrm{~m}, 10 \mathrm{H}), 1.59-1.49(\mathrm{~m}, 2 \mathrm{H}) \mathrm{ppm}$; HPLC-MS (ESI) m/z calcd. For $\mathrm{C}_{20} \mathrm{H}_{28} \mathrm{~N}_{2} \mathrm{O}_{2}[\mathrm{M}+1]=329.22$. Found: 329.30 .<smiles>CC(=O)N1C2(CCOCC2)COC12CCN(Cc1ccccc1)CC2</smiles>

1-\{11-benzyl-3,14-dioxa-7,11-diazadispiro[5.1.5.$\left.^{8}{ }^{6}\right]$ pentadecan-7-yl\} ethan-1one 17\{5\}: Colorless oil, 65\% yield. ${ }^{1} \mathrm{H}$ NMR (300 MHz, $\left.\mathrm{CDCl}_{3}\right) \delta 7.29-7.17(\mathrm{~m}, 5 \mathrm{H}), 3.93$ $-3.89(\mathrm{~m}, 4 \mathrm{H}), 3.46(\mathrm{~s}, 2 \mathrm{H}), 3.28(\mathrm{t}, 2 \mathrm{H}, J=11.9 \mathrm{~Hz}), 2.72-2.66(\mathrm{~m}, 2 \mathrm{H}), 2.16(\mathrm{~s}, 3 \mathrm{H}), 1.67$ - $1.52(\mathrm{~m}, 2 \mathrm{H}), 1.39-1.26(\mathrm{~m}, 2 \mathrm{H})$ ppm; HPLC-MS (ESI) m/z calcd. For $\mathrm{C}_{20} \mathrm{H}_{28} \mathrm{~N}_{2} \mathrm{O}_{3}[\mathrm{M}+$ 1] $=345.21$ Found: 345.20 .<smiles>CC1(C)COC2(CCN(Cc3ccccc3)CC2)N1C(=O)c1ccccc1</smiles>

4-benzoyl-8-benzyl-3,3-dimethyl-1-oxa-4,8-diazaspiro[4.5]decane $17\{8\}$ : White solid, 76\% yield. ${ }^{1} \mathrm{H}$ NMR (300 MHz, $\left.\mathrm{CDCl}_{3}\right) \delta 7.32-7.20(\mathrm{~m}, 10 \mathrm{H}), 3.64(\mathrm{~s}, 2 \mathrm{H})$, 
$3.45(\mathrm{~s}, 2 \mathrm{H}), 2.77-2.67(\mathrm{~m}, 4 \mathrm{H}), 2.31(\mathrm{t}, 2 \mathrm{H}, J=11.6 \mathrm{~Hz}), 1.51$ (d. $2 \mathrm{H}, J=11.6 \mathrm{~Hz}), 1.13$ (s, $6 \mathrm{H})$ ppm; HRMS (ESI-MS) calcd. for $\mathrm{C}_{23} \mathrm{H}_{28} \mathrm{~N}_{2} \mathrm{O}_{2}[\mathrm{M}+\mathrm{H}]^{+}=365.2151$. Found: 365.2148.

For the de-benzylation conditions (step e) see above.

\section{Examples of de-benzylated products}

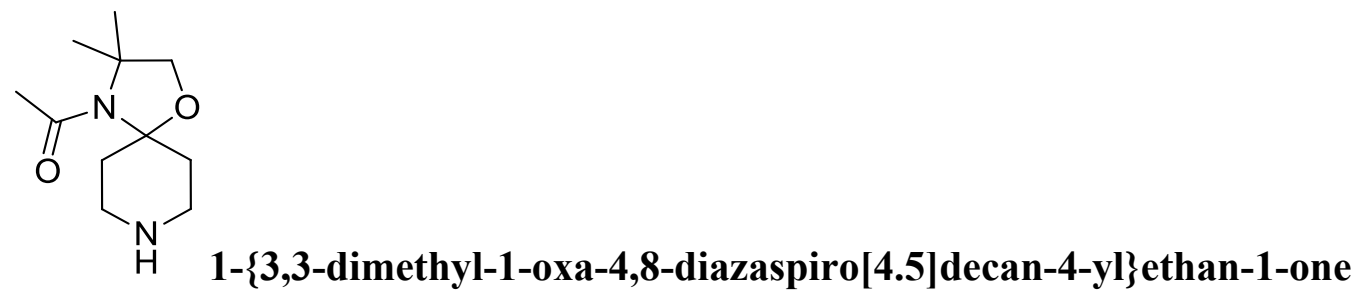

$18\{1\}$ :

Orange oil, 99\% yield. ${ }^{1} \mathrm{H}$ NMR $\left(300 \mathrm{MHz}, \mathrm{CDCl}_{3}\right) \delta 3.76(\mathrm{~s}, 2 \mathrm{H}), 3.06-2.87(\mathrm{~m}, 4 \mathrm{H}), 2.77$ (td, 2H, $J=5.1 \mathrm{~Hz}, J=12.6 \mathrm{~Hz}, J=12.7 \mathrm{~Hz}), 2.34-2.23(\mathrm{~m}, 2 \mathrm{H}), 2.17(\mathrm{~s}, 3 \mathrm{H}), 1.47(\mathrm{~d}, 6 \mathrm{H}$, $J=10.0 \mathrm{~Hz}$ ) ppm; HPLC-MS (ESI) $\mathrm{m} / \mathrm{z}$ calcd. For $\mathrm{C}_{11} \mathrm{H}_{20} \mathrm{~N}_{2} \mathrm{O}_{2}[\mathrm{M}+1]=213.15$. Found: 213.20<smiles>CC(=O)N1C2(CCCC2)CCC12CCNCC2</smiles>

Brown oil, 99\% yield. ${ }^{1} \mathrm{H}$ NMR $\left(300 \mathrm{MHz}, \mathrm{CDCl}_{3}\right) \delta 3.65$ (s, 2H), $3.30-3.19(\mathrm{~m}, 1 \mathrm{H}), 3.03$ $-2.67(\mathrm{~m}, 4 \mathrm{H}), 2.23-2.18(\mathrm{~m}, 1 \mathrm{H}), 2.10(\mathrm{~d}, 3 \mathrm{H}, J=17.3 \mathrm{~Hz}), 1.76-1.72(\mathrm{~m}, 4 \mathrm{H}), 1.51-$ $1.37(\mathrm{~m}, 4 \mathrm{H})$ ppm; HPLC-MS (ESI) m/z calcd. For $\mathrm{C}_{13} \mathrm{H}_{22} \mathrm{~N}_{2} \mathrm{O}_{2}[\mathrm{M}+1]=239.17$. Found: 239.30<smiles>CC(=O)N1C2(CCCCC2)COC12CCNCC2</smiles>

White solid, 99\% yield. ${ }^{1} \mathrm{H}$ NMR (300 MHz, $\left.\mathrm{CDCl}_{3}\right) \delta 3.86(\mathrm{~s}, 2 \mathrm{H}), 2.96-2.73(\mathrm{~m}, 4 \mathrm{H}), 2.73$ (s, 4H), $2.14(\mathrm{~s}, 3 \mathrm{H}), 1.94-1.92(\mathrm{~m}, 2 \mathrm{H}), 1.67(\mathrm{~d}, 6 \mathrm{H}, J=12.3 \mathrm{~Hz}), 1.42-1.10(\mathrm{~m}, 6 \mathrm{H})$ ppm; HRMS (ESI-MS) calcd. for $\mathrm{C}_{14} \mathrm{H}_{24} \mathrm{~N}_{2} \mathrm{O}_{2}[\mathrm{M}+\mathrm{H}]^{+}=253.1834$. Found: 253.1826. 
<smiles>CC1(C)COC2(CCNCC2)N1C(=O)c1ccccc1</smiles>

(3,3-dimethyl-1-oxa-4,8-diazaspiro[4.5]decan-4-yl)(phenyl)methanone

$\mathbf{1 8}\{\mathbf{8}\}$ : White solid, 97\% yield. ${ }^{1} \mathrm{H}$ NMR $\left(300 \mathrm{MHz}, \mathrm{CDCl}_{3}\right) \delta 7.38-7.36(\mathrm{~m}, 2 \mathrm{H}), 7.29-$ $7.26(\mathrm{~m}, 2 \mathrm{H}), 3.72$ (s, 2H), $3.02-2.87(\mathrm{~m}, 4 \mathrm{H}), 2.19$ (s, 2H), 1.64 (d, 2H, J=11.6 Hz), 1.17 $(\mathrm{s}, 6 \mathrm{H})$ ppm. HRMS (ESI-MS) calcd. for $\mathrm{C}_{16} \mathrm{H}_{22} \mathrm{~N}_{2} \mathrm{O}_{2}[\mathrm{M}+\mathrm{H}]^{+}=275.1681$. Found: 275.1677 .

\section{Examples of final diversification products (bis-spiro-oxazolidine library)}

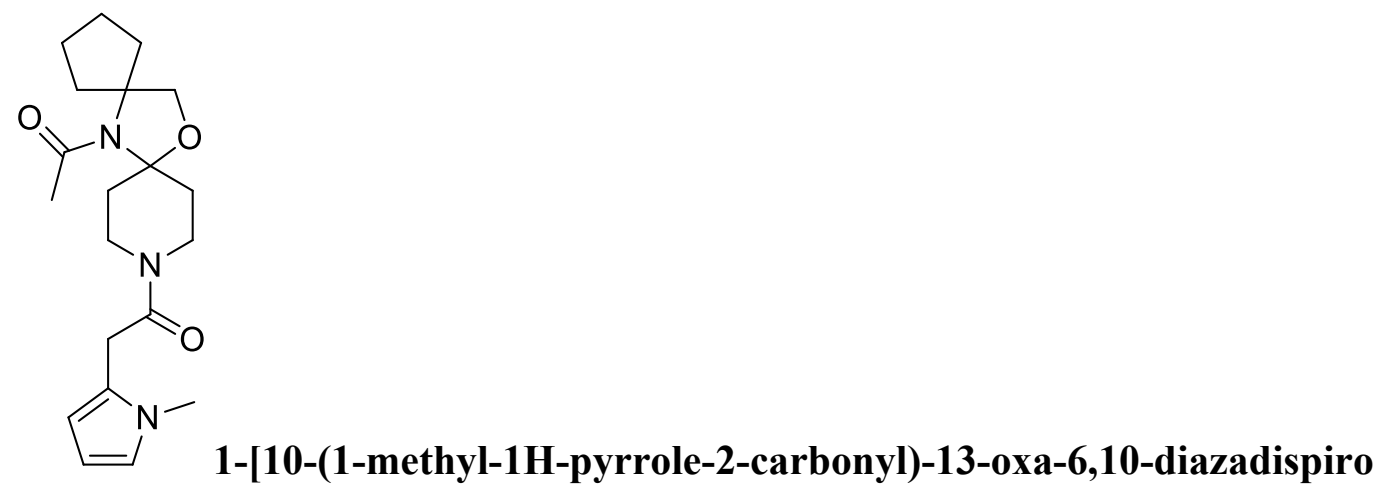

[4.1.5.$^{7}{ }^{5}$ ]tetradecan-6-yl]ethan-1-one 19\{3.1\}: Brown oil. ${ }^{1} \mathrm{H}$ NMR $\left(300 \mathrm{MHz}, \mathrm{CDCl}_{3}\right) \delta$ $6.66(\mathrm{~s}, 1 \mathrm{H}), 6.33(\mathrm{~d}, 1 \mathrm{H}, J=3.7 \mathrm{~Hz}), 6.05(\mathrm{~s}, 1 \mathrm{H}), 4.43(\mathrm{~d}, 2 \mathrm{H}, J=8.2 \mathrm{~Hz}), 3.77(\mathrm{~s}, 4 \mathrm{H})$, $3.25-3.13(\mathrm{~m}, 2 \mathrm{H}), 2.89(\mathrm{t}, 2 \mathrm{H}, J=12.6 \mathrm{~Hz}), 2.12(\mathrm{~s}, 3 \mathrm{H}), 2.00(\mathrm{~s}, 3 \mathrm{H}), 1.83(\mathrm{~s}, 4 \mathrm{H}), 1.59-$ $1.50(\mathrm{~m}, 4 \mathrm{H})$ ppm. HRMS (ESI-MS) calcd. for $\mathrm{C}_{19} \mathrm{H}_{27} \mathrm{~N}_{3} \mathrm{O}_{3}[\mathrm{M}+\mathrm{H}]^{+}=346.2052$. Found: 346.2049<smiles>CC(=O)N1C2(CCCCC2)COC12CCN(C(=O)C(C)C)CC2</smiles>

1-\{7-acetyl-15-oxa-3,7-diazadispiro[5.1.5 $\left.{ }^{8} .2^{6}\right]$ pentadecan-3-yl\}-2-methyl

propan-1-one 19\{4.1\}: Yellowish oil. ${ }^{1} \mathrm{H}$ NMR (300 $\left.\mathrm{MHz}, \mathrm{CDCl}_{3}\right) \delta 4.58(\mathrm{~d}, 1 \mathrm{H}, J=9.3$ Hz), $3.83(\mathrm{~d}, 1 \mathrm{H}, J=12.5 \mathrm{~Hz}), 3.73(\mathrm{~s}, 2 \mathrm{H}), 3.29$ (t, 1H, $J=12.8 \mathrm{~Hz}), 2.84-2.76(\mathrm{~m}, 4 \mathrm{H})$, 
$2.10(\mathrm{~s}, 3 \mathrm{H}), 1.95-1.83(\mathrm{~m}, 7 \mathrm{H}), 1.58-1.49(\mathrm{~m}, 5 \mathrm{H}), 1.15-1.09(\mathrm{~m}, 6 \mathrm{H})$ ppm. HRMS (ESI-MS) calcd. for $\mathrm{C}_{18} \mathrm{H}_{30} \mathrm{~N}_{2} \mathrm{O}_{3}[\mathrm{M}+\mathrm{H}]^{+}=323.2256$. Found: 323.2250.<smiles>CC(=O)N1C2(CCCC2)COC12CCN(Cc1c[nH]cn1)CC2</smiles>

tetradecan-6-yl\} ethan-1-one 20\{3.1\}: Yellow oil. ${ }^{1} \mathrm{H}$ NMR $\left(300 \mathrm{MHz}, \mathrm{CDCl}_{3}\right) \delta 8.55(\mathrm{~s}$, 1H), $7.12(\mathrm{~s}, 1 \mathrm{H}), 4.07(\mathrm{~s}, 2 \mathrm{H}), 3.72(\mathrm{~s}, 2 \mathrm{H}), 3.22-3.10(\mathrm{~m}, 4 \mathrm{H}), 2.97-2.88(\mathrm{~m}, 2 \mathrm{H}), 2.13$ $(\mathrm{s}, 2 \mathrm{H}), 1.83-1.77(\mathrm{~m}, 4 \mathrm{H}), 1.57-1.44(\mathrm{~m}, 4 \mathrm{H})$ ppm. HRMS (ESI-MS) calcd. for $\mathrm{C}_{17} \mathrm{H}_{26} \mathrm{~N}_{4} \mathrm{O}_{2}[\mathrm{M}+\mathrm{H}]^{+}=319.2056$. Found: 319.2052.<smiles>CC(=O)N1C2(CCOCC2)COC12CCN(Cc1c(F)cccc1Cl)CC2</smiles>

\section{1-\{11-[(2-chloro-6-fluorophenyl)methyl]-3,14-dioxa-7,11-diazadispiro}

[5.1.5 ${ }^{8} .2^{6}$ ]pentadecan-7-yl\} ethan-1-one 20\{5.1\}: White solid. ${ }^{1} \mathrm{H}$ NMR $\left(300 \mathrm{MHz}, \mathrm{CDCl}_{3}\right) \delta$ $7.13(\mathrm{~s}, 2 \mathrm{H}), 6.97-6.84(\mathrm{~m}, 1 \mathrm{H}), 3.73(\mathrm{~s}, 2 \mathrm{H}), 3.59(\mathrm{~d}, 2 \mathrm{H}, J=14.9 \mathrm{~Hz}), 2.93-2.83(\mathrm{~m}$, 2H), $2.48(\mathrm{t}, 2 \mathrm{H}, J=11.5 \mathrm{~Hz}), 2.27-2.20(\mathrm{~m}, 2 \mathrm{H}), 2.06(\mathrm{t}, 3 \mathrm{H}, J=11.2 \mathrm{~Hz}), 1.85-1.61(\mathrm{~m}$, $6 \mathrm{H}), 1.49-1.36(\mathrm{~m}, 4 \mathrm{H}) \mathrm{ppm}$. HRMS (ESI-MS) calcd. for $\mathrm{C}_{20} \mathrm{H}_{26} \mathrm{ClFN}_{2} \mathrm{O}_{3}[\mathrm{M}+\mathrm{H}]^{+}=$ 397.1616. Found: 397.1610.<smiles>COc1ccc(OC)c(S(=O)(=O)N2CCC3(CC2)OCC2(CCCC2)N3C(C)=O)c1</smiles> 
[4.1.5 ${ }^{7} \mathbf{2}^{5}$ ]tetradecan-6-yl]ethan-1-one 21\{3.1\}: Orange oil. ${ }^{1} \mathrm{H}$ NMR $\left(300 \mathrm{MHz}, \mathrm{CDCl}_{3}\right) \delta$ 7.37 (s, 1H), 6.98 (d, 1H, $J=6.8 \mathrm{~Hz}), 6.89$ (d, $1 \mathrm{H}, J=8.9 \mathrm{~Hz}), 3.82$ (s, 3H), $3.72(\mathrm{~s}, 3 \mathrm{H})$, 3.57 (d, 2H, $J=13.9 \mathrm{~Hz}), 2.96-2.76(\mathrm{~m}, 4 \mathrm{H}), 2.23-2.19(\mathrm{~m}, 2 \mathrm{H}), 2.03$ (s, 3H), $1.94-1.72$ $(\mathrm{m}, 6 \mathrm{H}), 1.50-1.36(\mathrm{~m}, 4 \mathrm{H}) \mathrm{ppm}$; HPLC-MS (ESI) m/z calcd. For $\mathrm{C}_{21} \mathrm{H}_{30} \mathrm{~N}_{2} \mathrm{O}_{6} \mathrm{~S}[\mathrm{M}+1]=$ 439.18. Found: 439.20 .

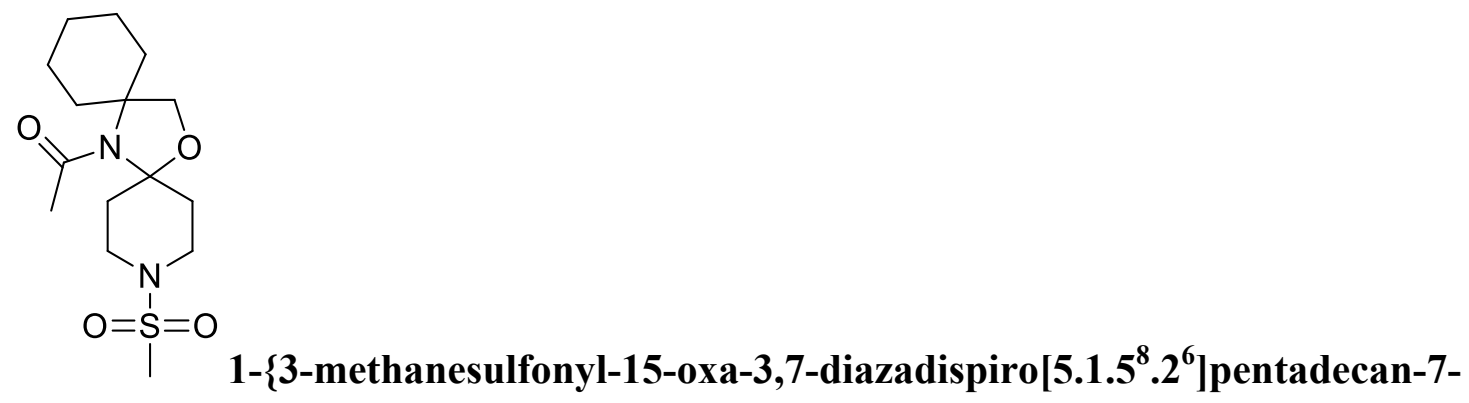

yl\}ethan-1-one 21\{4.1\}: White solid. ${ }^{1} \mathrm{H}$ NMR $\left(300 \mathrm{MHz}, \mathrm{CDCl}_{3}\right) \delta 3.71-3.66(\mathrm{~m}, 4 \mathrm{H})$, 2.97 (d, 3H, $J=7.5 \mathrm{~Hz}), 2.79$ (s, 2H), $2.37-2.16(\mathrm{~m}, 1 \mathrm{H}), 2.06(\mathrm{~s}, 3 \mathrm{H}), 1.94-1.77(\mathrm{~m}, 8 \mathrm{H})$, $1.52-1.44(\mathrm{~m}, 5 \mathrm{H})$ ppm. HRMS (ESI-MS) calcd. for $\mathrm{C}_{15} \mathrm{H}_{26} \mathrm{~N}_{2} \mathrm{O}_{4} \mathrm{~S}[\mathrm{M}+\mathrm{H}]^{+}=331.1613$. Found: 331.1607.<smiles>CC(=O)N1C2(CCCC2)COC12CCN(C(=O)Nc1c(C)cccc1Cl)CC2</smiles>

\section{6-acetyl-N-(2,6-dichlorophenyl)-13-oxa-6,10-diazadispiro[4.1.5 $\left..2^{7}\right]$}

tetradecane-10-carboxamide 22\{3.1\}: Yellow oil. ${ }^{1} \mathrm{H}$ NMR $\left(300 \mathrm{MHz}, \mathrm{CDCl}_{3}\right) \delta 7.31$ (d, $2 \mathrm{H}, J=7.8 \mathrm{~Hz}$ ), $7.12-7.03(\mathrm{~m}, 1 \mathrm{H}), 6.19(\mathrm{br} \mathrm{s}, 1 \mathrm{H}), 4.04$ (d, 2H, $J=12.6 \mathrm{~Hz}), 3.74(\mathrm{~s}, 2 \mathrm{H})$, $3.25(\mathrm{t}, 2 \mathrm{H}, J=12.9 \mathrm{~Hz}), 2.94(\mathrm{t}, 2 \mathrm{H}, J=10.8 \mathrm{~Hz}), 2.29(\mathrm{~d}, 2 \mathrm{H}, J=10.6 \mathrm{~Hz}), 1.89-1.76(\mathrm{~m}$, $4 \mathrm{H}), 1.57-1.45(\mathrm{~m}, 4 \mathrm{H}) \mathrm{ppm}$. HRMS (ESI-MS) calcd. for $\mathrm{C}_{20} \mathrm{H}_{25} \mathrm{Cl}_{2} \mathrm{~N}_{3} \mathrm{O}_{3}[\mathrm{M}+\mathrm{H}]^{+}=$ 426.1273. Found: 426.1270. 
<smiles>CC(=O)N1C2(CCCC2)COC12CCN(C(=O)Nc1ccc(OC(F)(F)F)cc1)CC2</smiles>

6-acetyl-N-[4-(trifluoromethoxy)phenyl]-13-oxa-6,10-diazadispiro[4.1.5 $\left..^{7} .^{5}\right]$ tetradecane-10-carboxamide 22\{3.2\}: White solid. ${ }^{1} \mathrm{H}$ NMR $\left(300 \mathrm{MHz}, \mathrm{CDCl}_{3}\right) \delta 7.35(\mathrm{~d}$, $2 \mathrm{H}, J=8.7 \mathrm{~Hz}), 7.11$ (d, 2H, $J=8.3 \mathrm{~Hz}), 6.61(\mathrm{br} \mathrm{s}, 1 \mathrm{H}), 3.97$ (d, 2H, $J=11.9 \mathrm{~Hz}), 3.74$ (s, 2H), 3.18 (t, $2 \mathrm{H}, J=12.2 \mathrm{~Hz}), 2.86(\mathrm{t}, 2 \mathrm{H}, J=12.4 \mathrm{~Hz}), 2.11(\mathrm{~s}, 3 \mathrm{H}), 2.00-1.95(\mathrm{~m}, 2 \mathrm{H})$, $1.83(\mathrm{~s}, 4 \mathrm{H}), 1.57-1.52(\mathrm{~m}, 4 \mathrm{H})$ ppm; HPLC-MS (ESI) m/z calcd. For $\mathrm{C}_{21} \mathrm{H}_{26} \mathrm{~F}_{3} \mathrm{~N}_{3} \mathrm{O}_{4}[\mathrm{M}+$ $1]=442.19$. Found: 442.20 


\section{Stability tests}

Stability tests were performed on intermediates $\mathbf{4}\{\mathbf{3}\}, \mathbf{5}\{\mathbf{3}\}, \mathbf{1 6}\{\mathbf{4}\}, \mathbf{1 7}\{\mathbf{4}\}$. The samples were analysed at the following time intervals:

$\mathrm{T}_{0}=$ starting point; $\mathrm{T}_{1}=4$ hours; $\mathrm{T}_{2}=16$ hours; $\mathrm{T}_{3}=40$ hours; $\mathrm{T}_{4}=72$ hours

Table 1. Stability of compounds $4\{3\}, 5\{3\}, 16\{4\}, 17\{4\}$

\begin{tabular}{|c|c|c|c|c|c|c|}
\hline Comnound & & & & ility & & \\
\hline Compound & Structure & $\mathbf{T}_{0}$ & $T_{1}$ & $T_{2}$ & $T_{3}$ & $\mathbf{T}_{4}$ \\
\hline $4\{3\}$ & & 100 & 100 & 100 & 100 & 100 \\
\hline $5\{3\}$ & & 100 & 95 & 89 & 83 & 78 \\
\hline $16\{4\}$ & & 100 & 100 & 100 & 100 & 100 \\
\hline $17\{4\}$ & & 100 & 80 & 65 & 52 & 37 \\
\hline
\end{tabular}


Analysis: $30 \mathrm{mg}$ of compound were dissolved in $3 \mathrm{~mL}$ of $\mathrm{THF}$ and $1 \mathrm{~mL}$ of $\mathrm{HCl}(2.5 \%$ in water, $\mathrm{pH}=2$ ). The resulting solutions were stirred at room temperature and monitored by UHPLC (UV absorbance: $250 \mathrm{~nm}$; Ionization method: DAD-TIC)

Figure 1. Sample stability profiles in acidic media $(\mathrm{pH}=2)$ for aminal $4\{3\}$ and hemiaminal ether $16\{4\}$ intermediates, and their acetylated counterparts, $5\{3\}$ and $17\{4\}$, respectively.

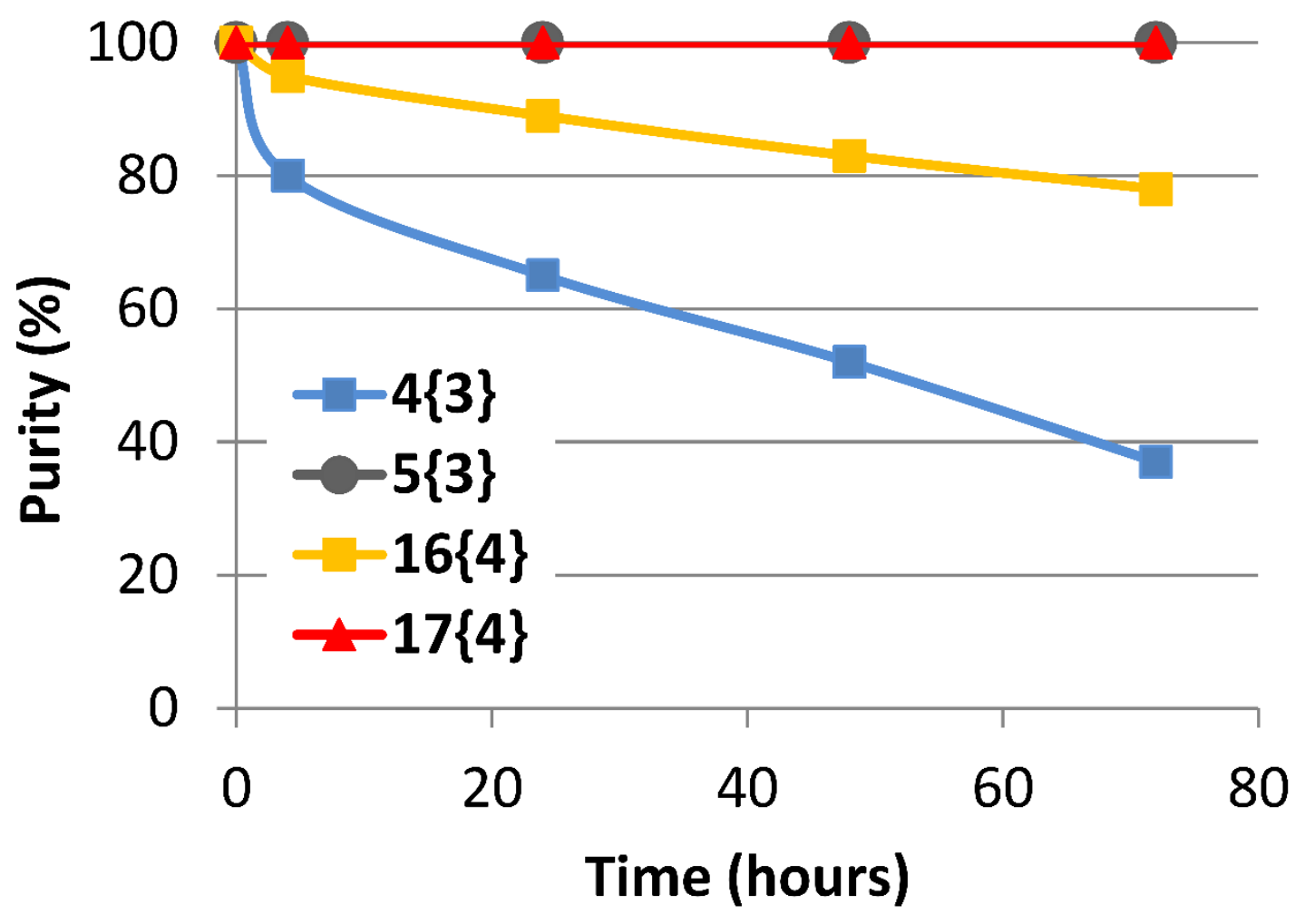


${ }^{1} \mathrm{H},{ }^{13} \mathrm{C}$ NMR, HPLC-MS (ESI) chromatograms and mass spectra for some representative compounds from the two libraries

${ }^{1} \mathrm{H}$ NMR (300 MHz, $\mathrm{CDCl}_{3}$ ) 4-amino-1-benzylpiperidine-4-carbonitrile (2) ${ }^{1}$

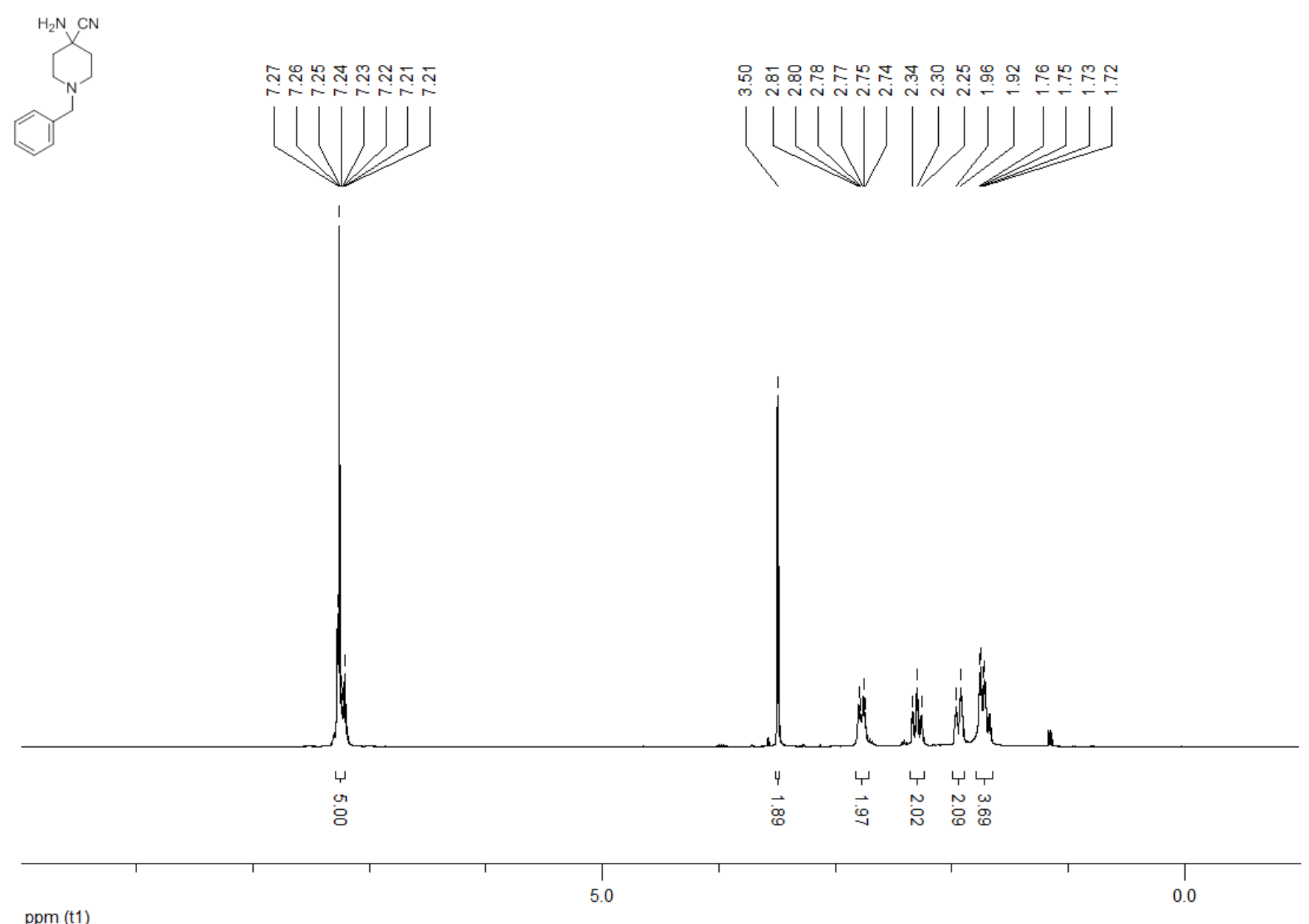


${ }^{1}$ H NMR (300 MHz, DMSO- $d_{6}$ ) 4-amino-1-benzylpiperidine-4-carboxamide (3) ${ }^{2}$
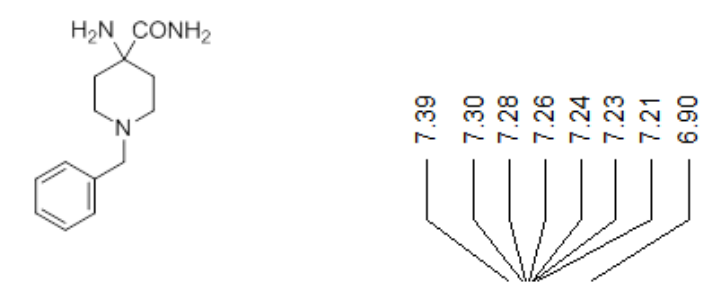

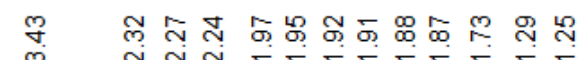
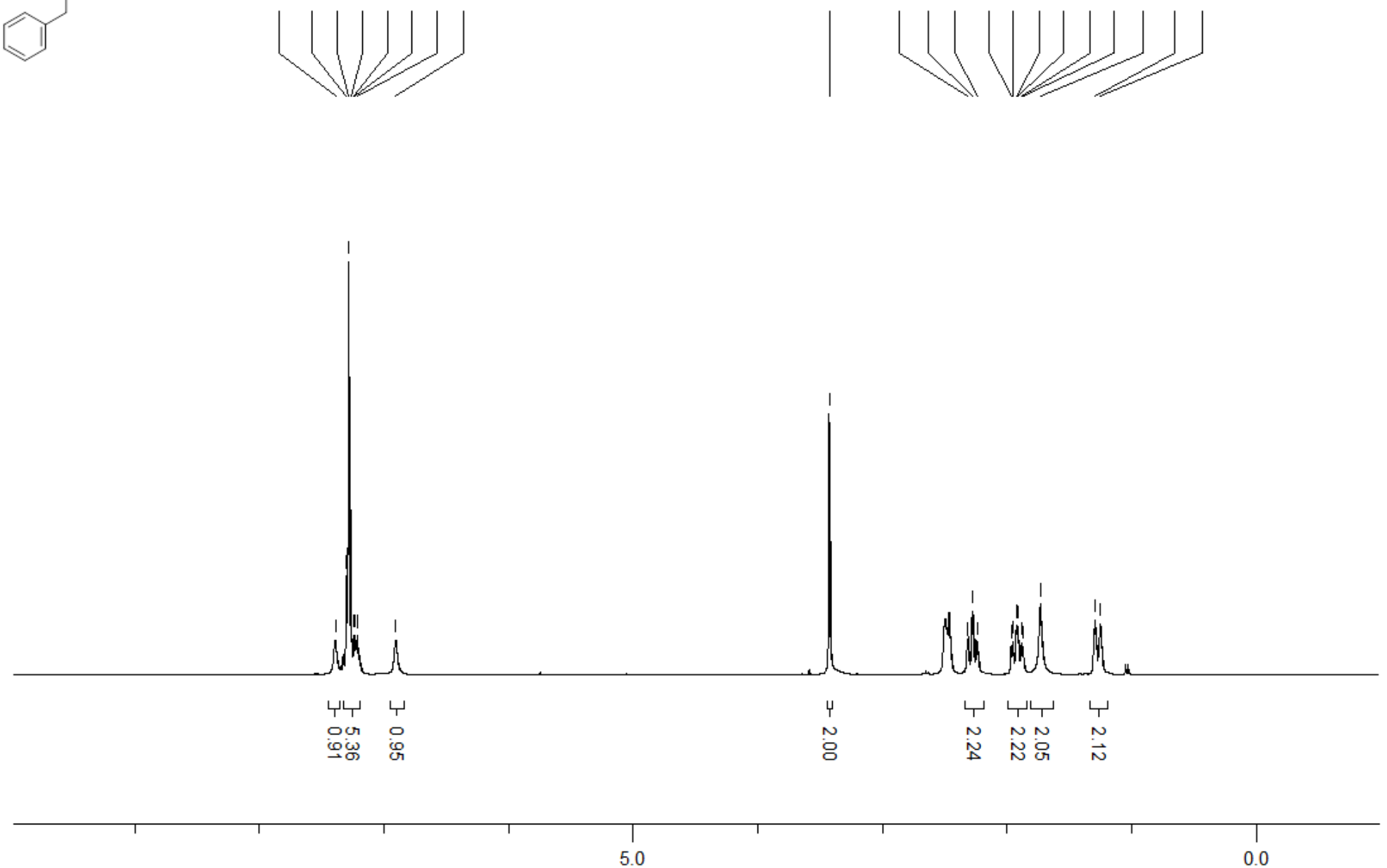

ppm (t1) 
${ }^{13}$ C NMR (100 MHz, DMSO- $d_{6}$ ) 4-amino-1-benzylpiperidine-4-carboxamide (3)
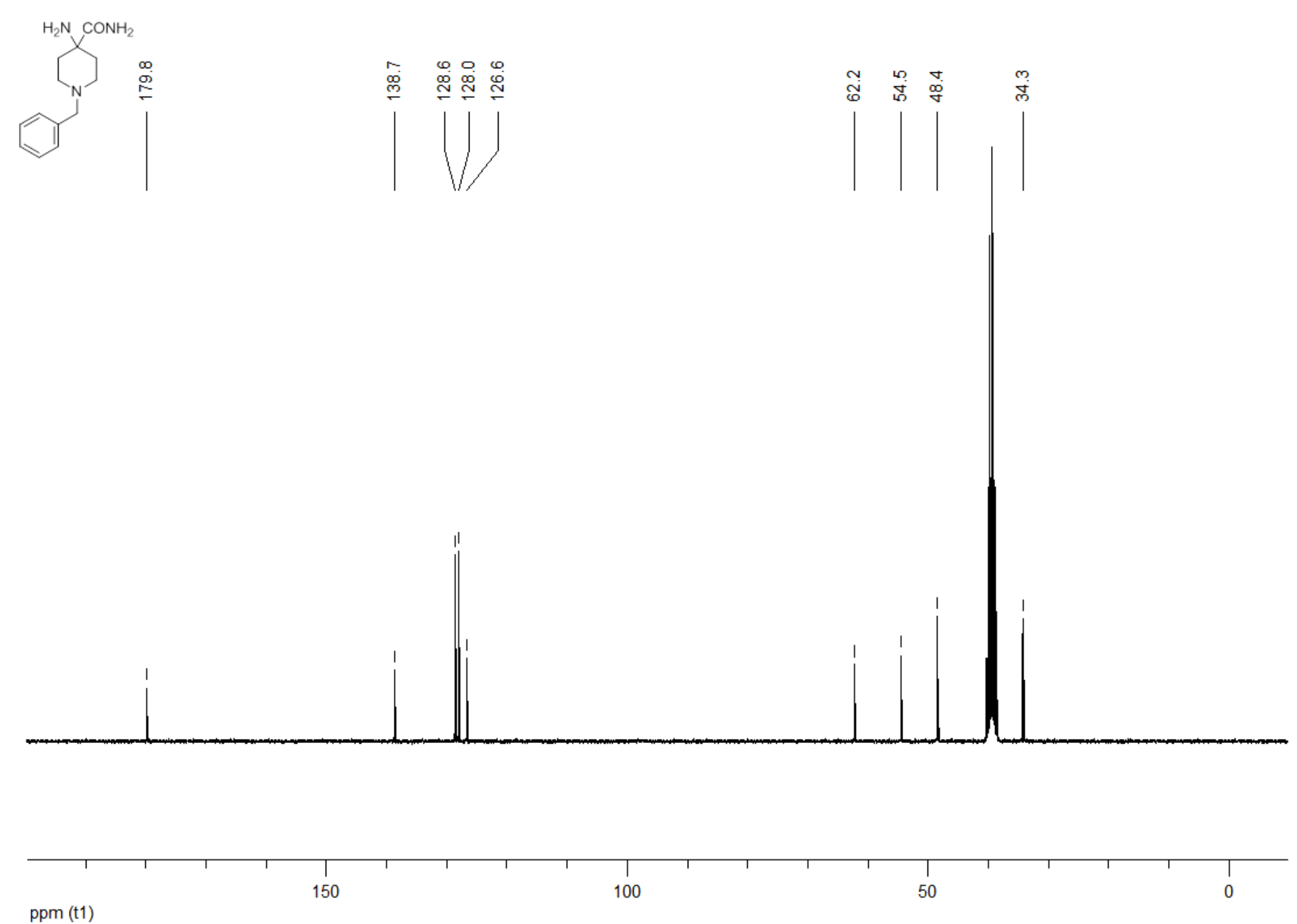
${ }^{1}$ H NMR (300 MHz, DMSO-d $d_{6}$ ) 8-benzyl-2,2-dimethyl-1,3,8-triazaspiro[4.5]decan-4-one 4\{1\}
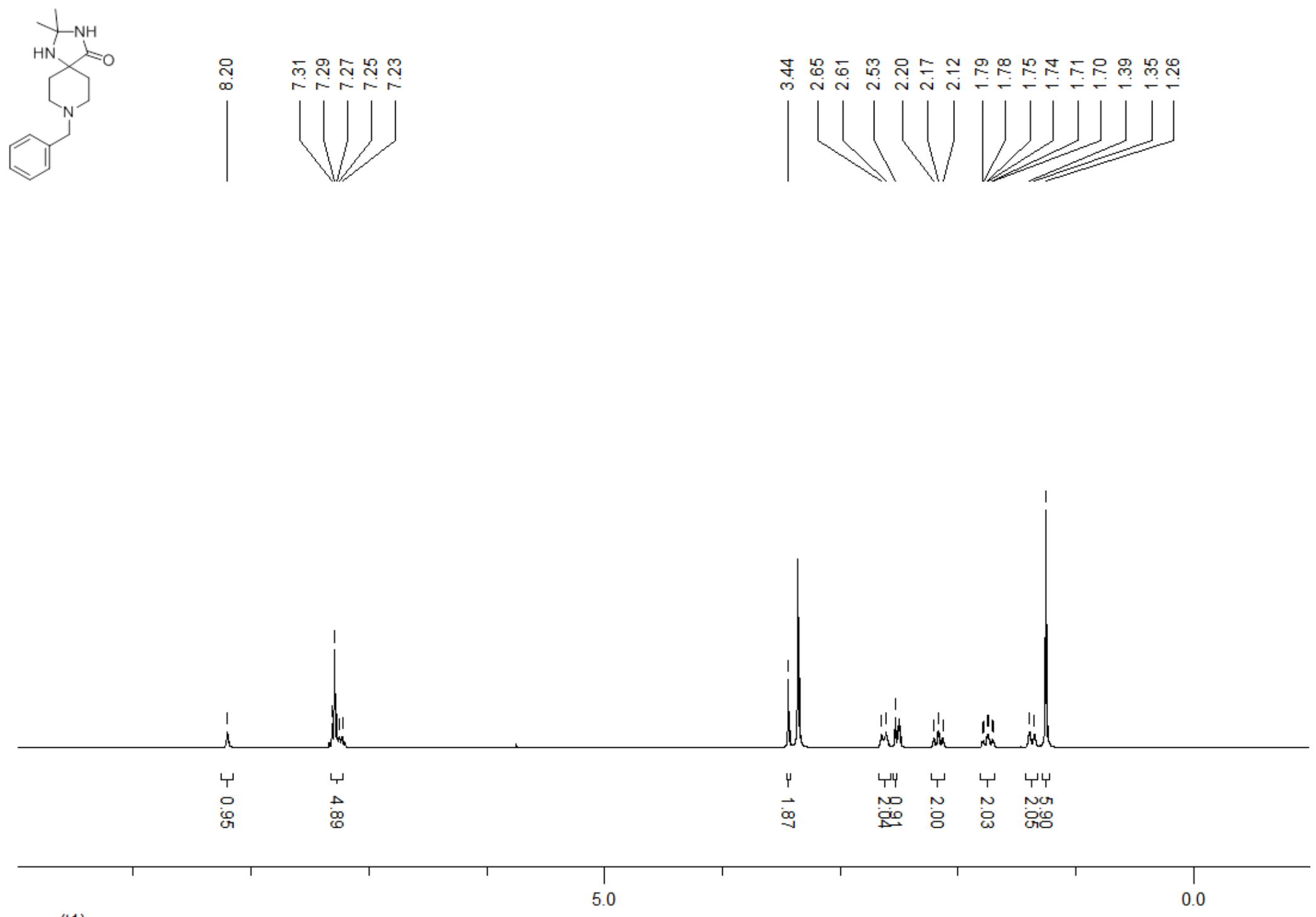

ppm (t1) 
${ }^{13}$ C NMR (100 MHz, DMSO- $\left.d_{6}\right)$ 8-benzyl-2,2-dimethyl-1,3,8-triazaspiro[4.5]decan-4-one 4\{1\}

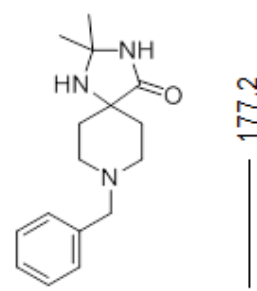

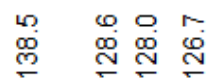
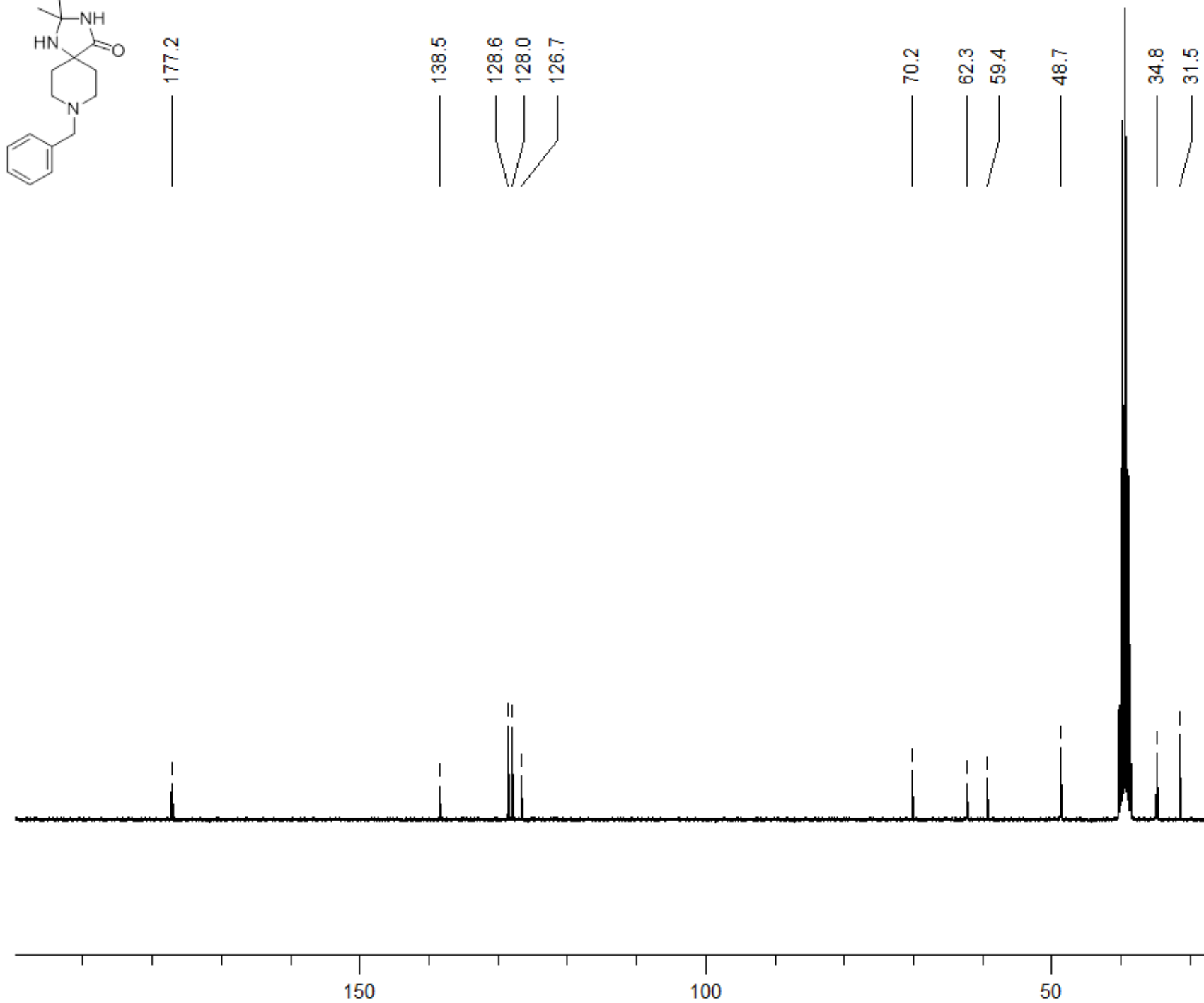

150

100

ppm (t1) 
${ }^{1} \mathrm{H}$ NMR (300 MHz, $\mathrm{CDCl}_{3}$ ) 9-benzyl-5,9,13-triazadispiro[3.1.5 $\left..^{6} .^{4}\right]$ tridecan-12-one $4\{2\}$
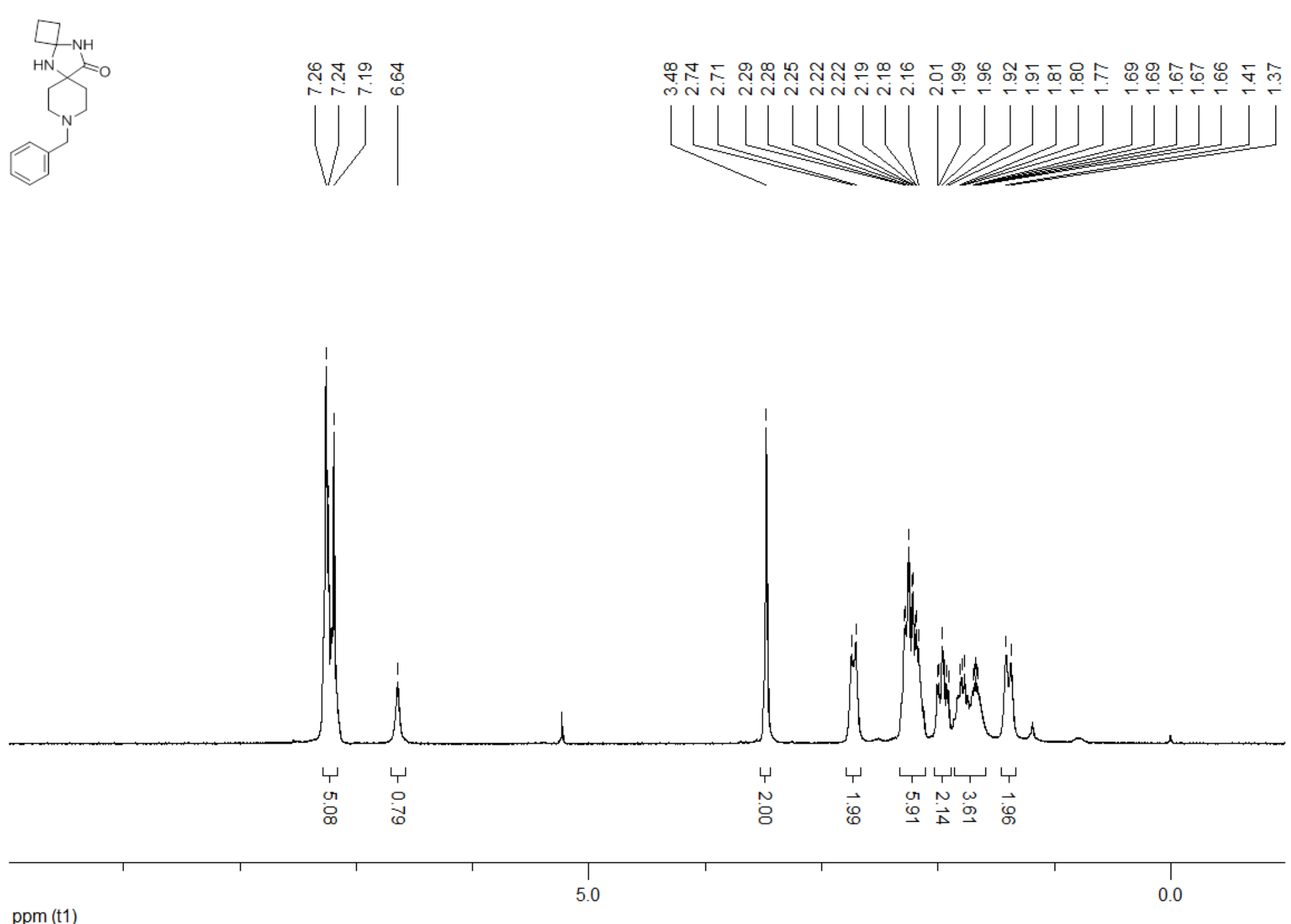
HPLC-MS (ESI) 9-benzyl-5,9,13-triazadispiro[3.1.5 $\left.{ }^{6} .^{4}\right]$ tridecan-12-one $4\{2\}$

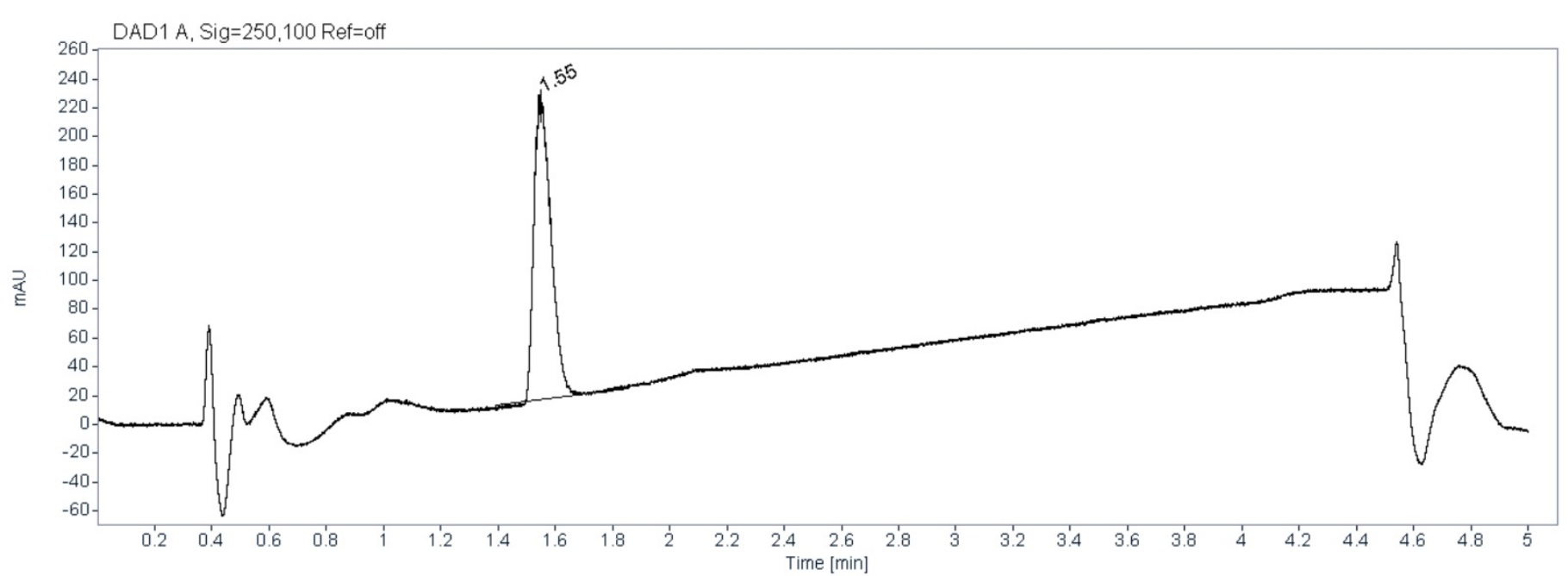

MSD1 SPC, MM-ES+APCI

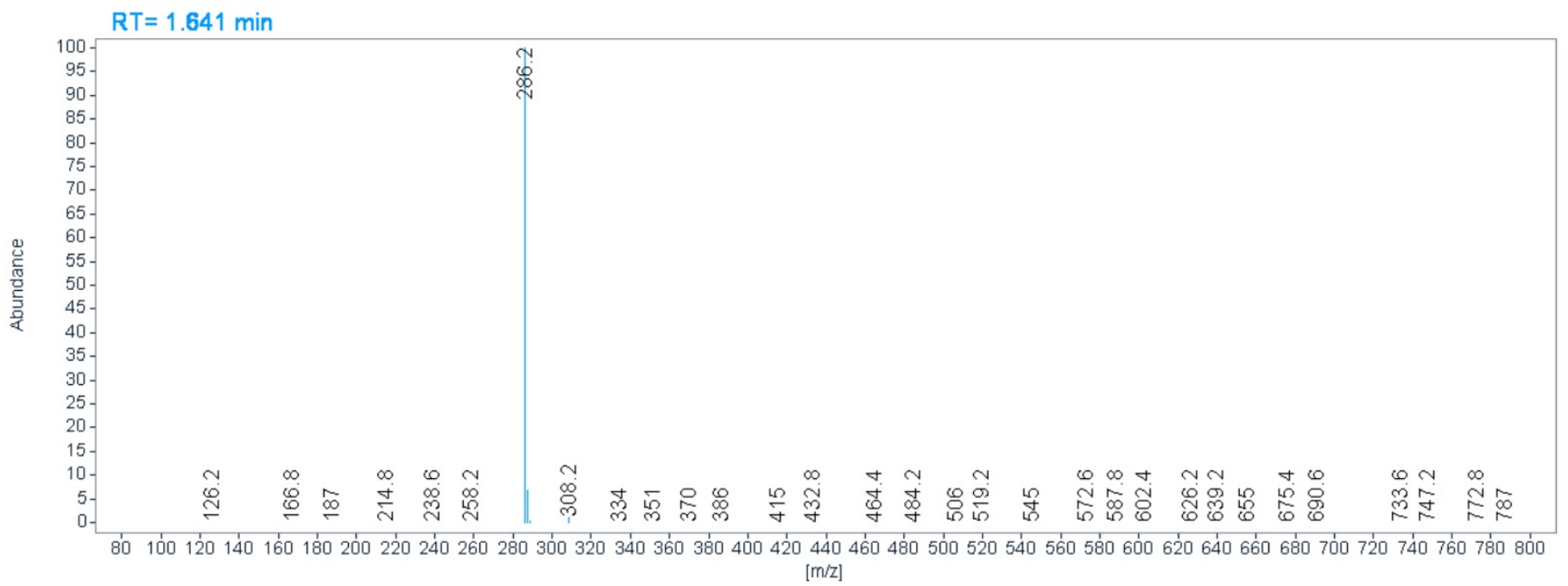


${ }^{1}$ H NMR (100 MHz, CDCl 3 ) 10-benzyl-6,10,14-triazadispiro[4.1.5.$\left.^{7} 2^{5}\right]$ tetradecan-13-one 4\{3\}

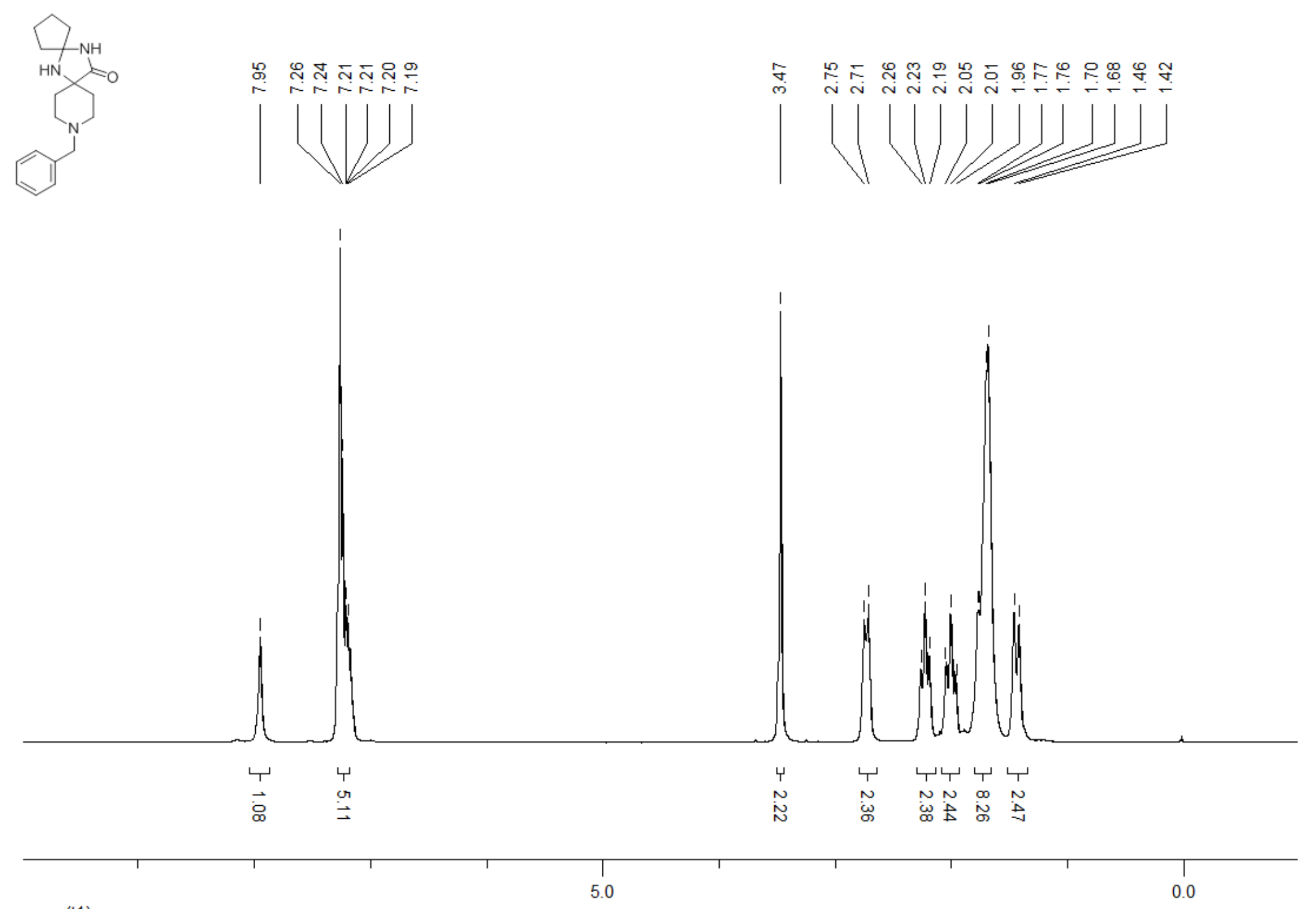

ppm (t1) 
${ }^{13} \mathrm{C}$ NMR (100 MHz, $\left.\mathrm{CDCl}_{3}\right)$ 10-benzyl-6,10,14-triazadispiro[4.1.5.$\left.^{7} \cdot 2^{5}\right]$ tetradecan-13-one $4\{3\}$
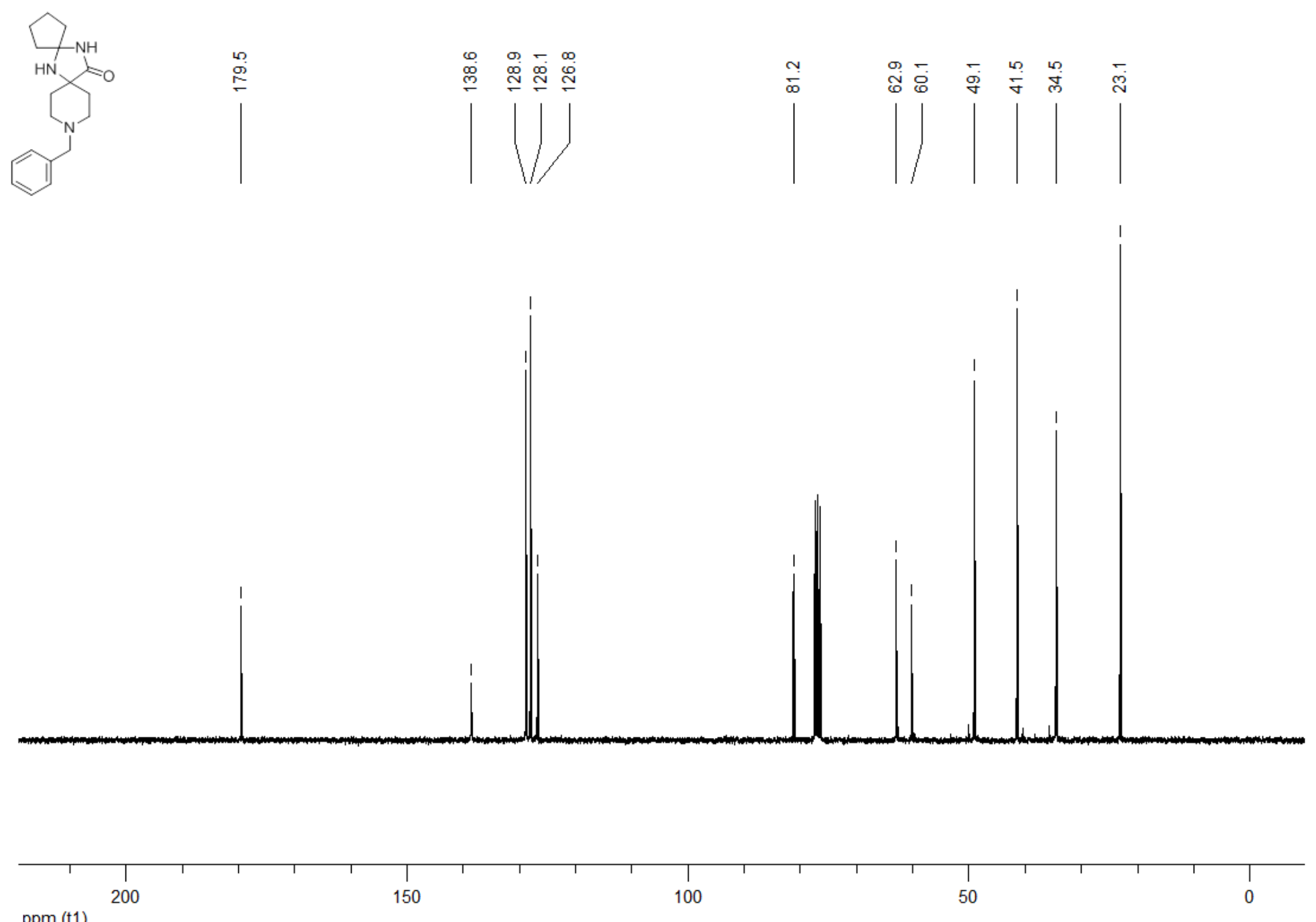
HPLC-MS (ESI) 10-benzyl-6,10,14-triazadispiro[4.1.5 $\left..^{7} .^{5}\right]$ tetradecan-13-one $4\{3\}$

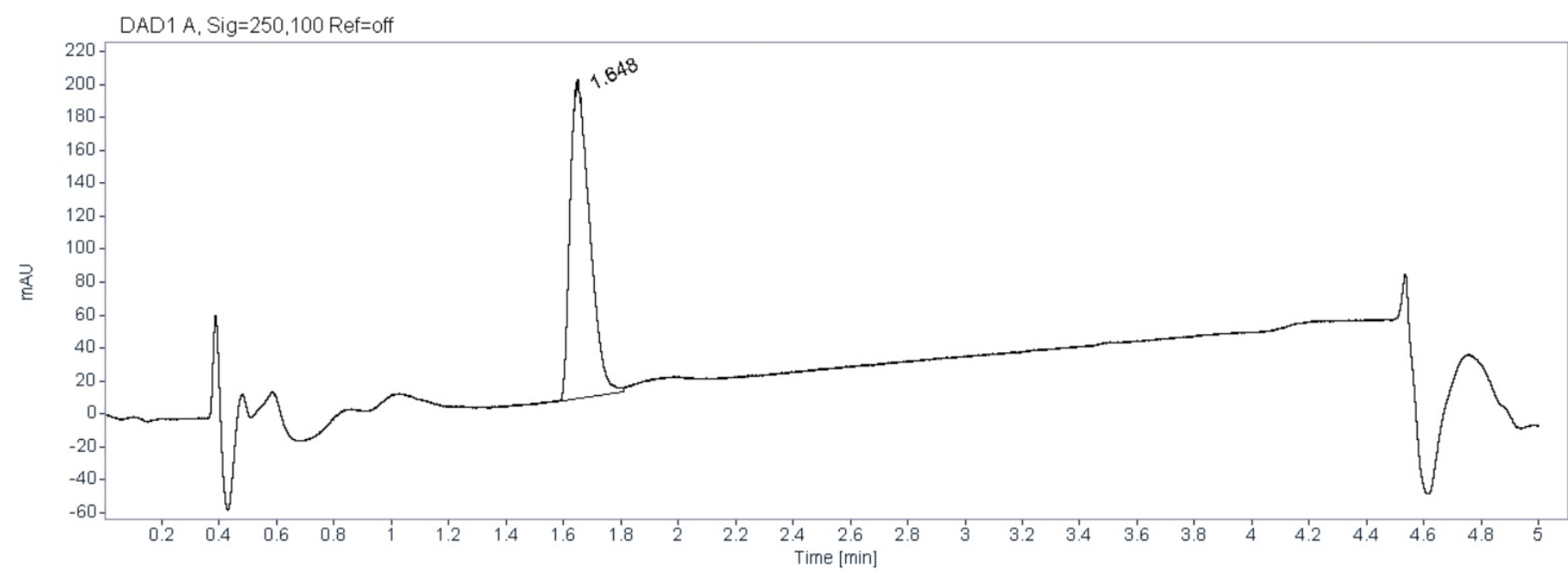

MSD1 SPC, MM-ES+APCI

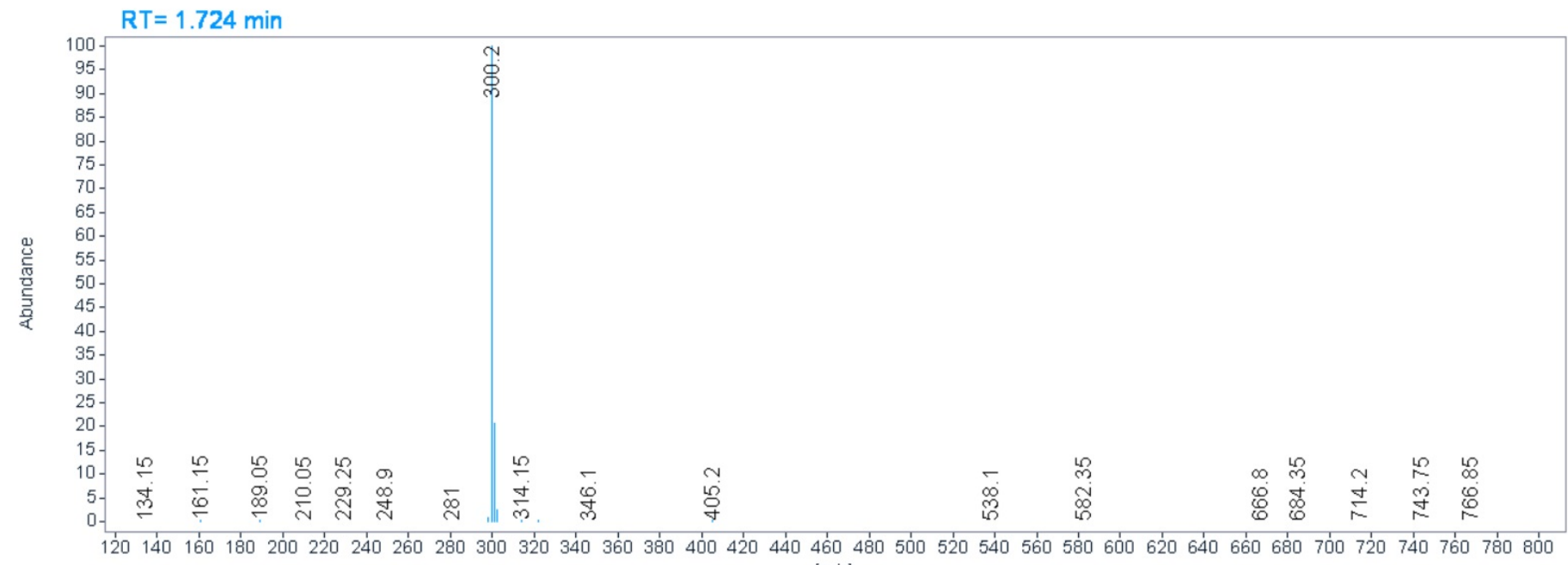


${ }^{1} \mathrm{H}$ NMR (300 MHz, CDCl 3 ) 3-benzyl-3,7,14-triazadispiro[5.1.5 $\left.5^{8} .^{6}\right]$ pentadecan-15-one $4\{4\}$
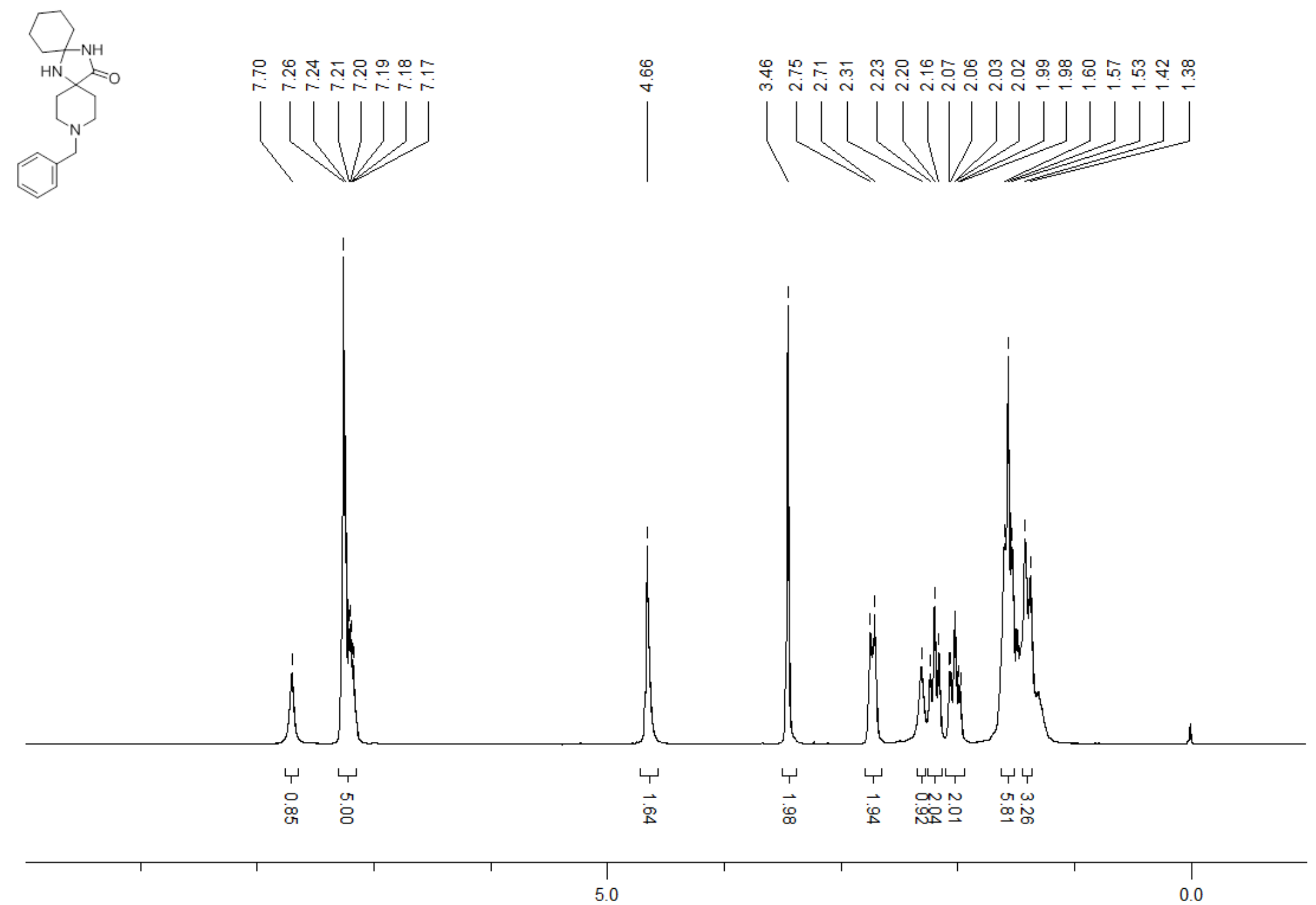

$\mathrm{ppm}(\mathrm{t} 1)$ 
${ }^{13} \mathrm{C}$ NMR (100 MHz, CDCl $)$ 3-benzyl-3,7,14-triazadispiro[5.1.5 $\left.{ }^{8} .2^{6}\right]$ pentadecan-15-one $4\{4\}$
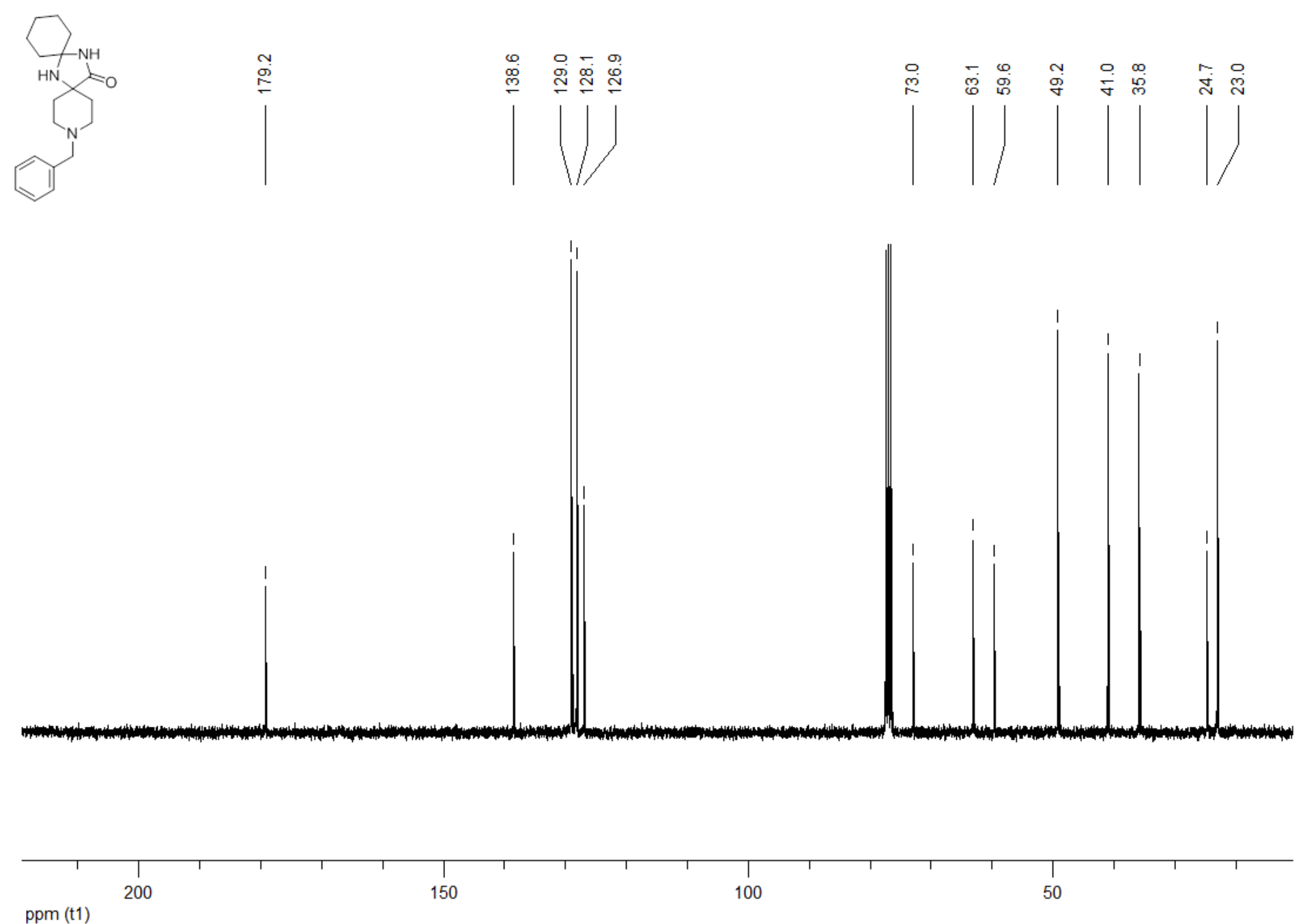
${ }^{1}$ H NMR (300 MHz, DMSO- $d_{6}$ ) 3-benzyl-11-oxa-3,7,14-triazadispiro[5.1.5 ${ }^{8} .^{6}$ ]pentadecan-15-one $4\{5\}$
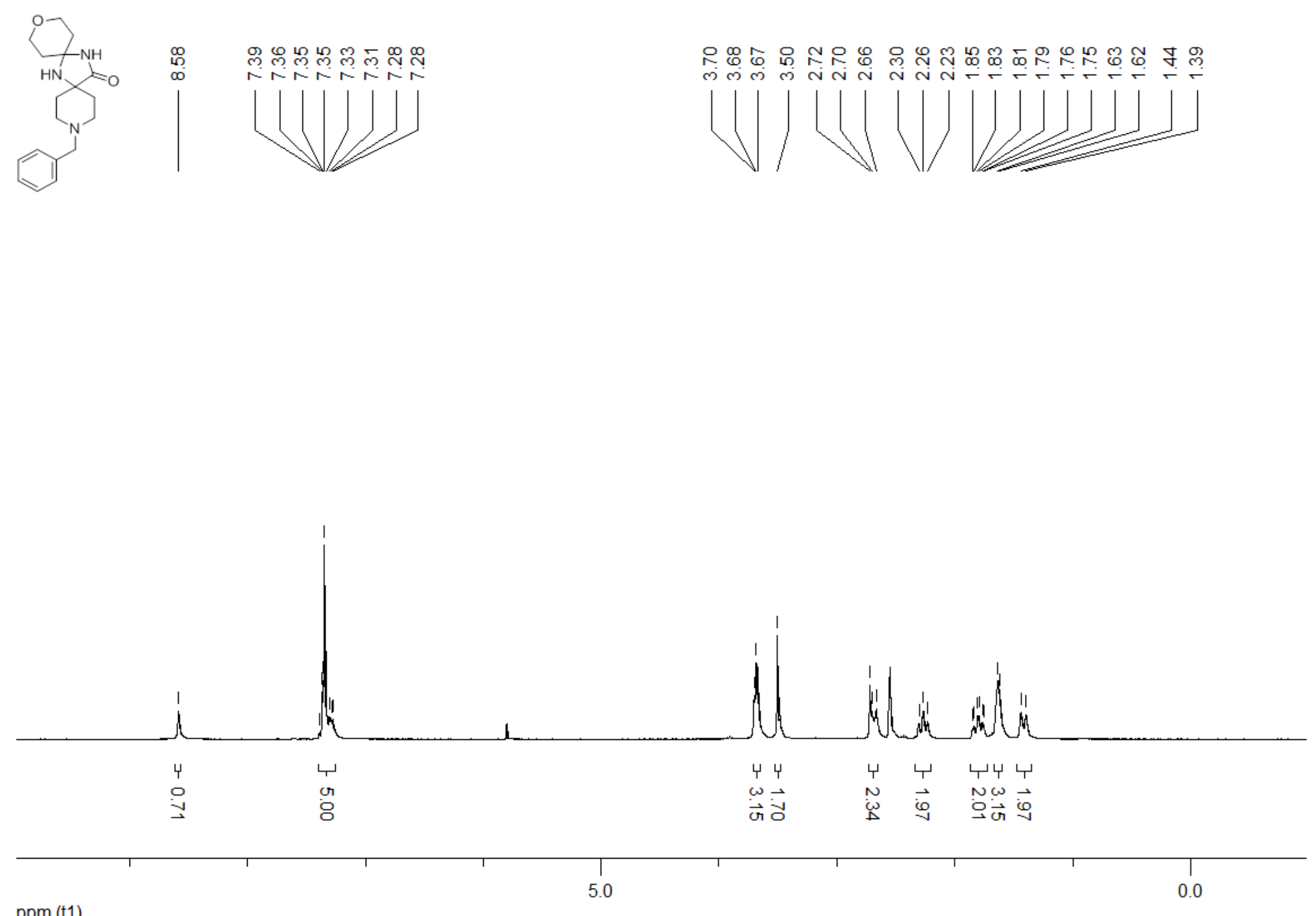

ppm (t1) 
${ }^{1}$ H NMR (300 MHz, DMSO-d $d_{6}$ 3-benzyl-11-methyl-3,7,11,14-tetraazadispiro[5.1.5 ${ }^{8} .2^{6}$ ]pentadecan-15-one $4\{6\}$
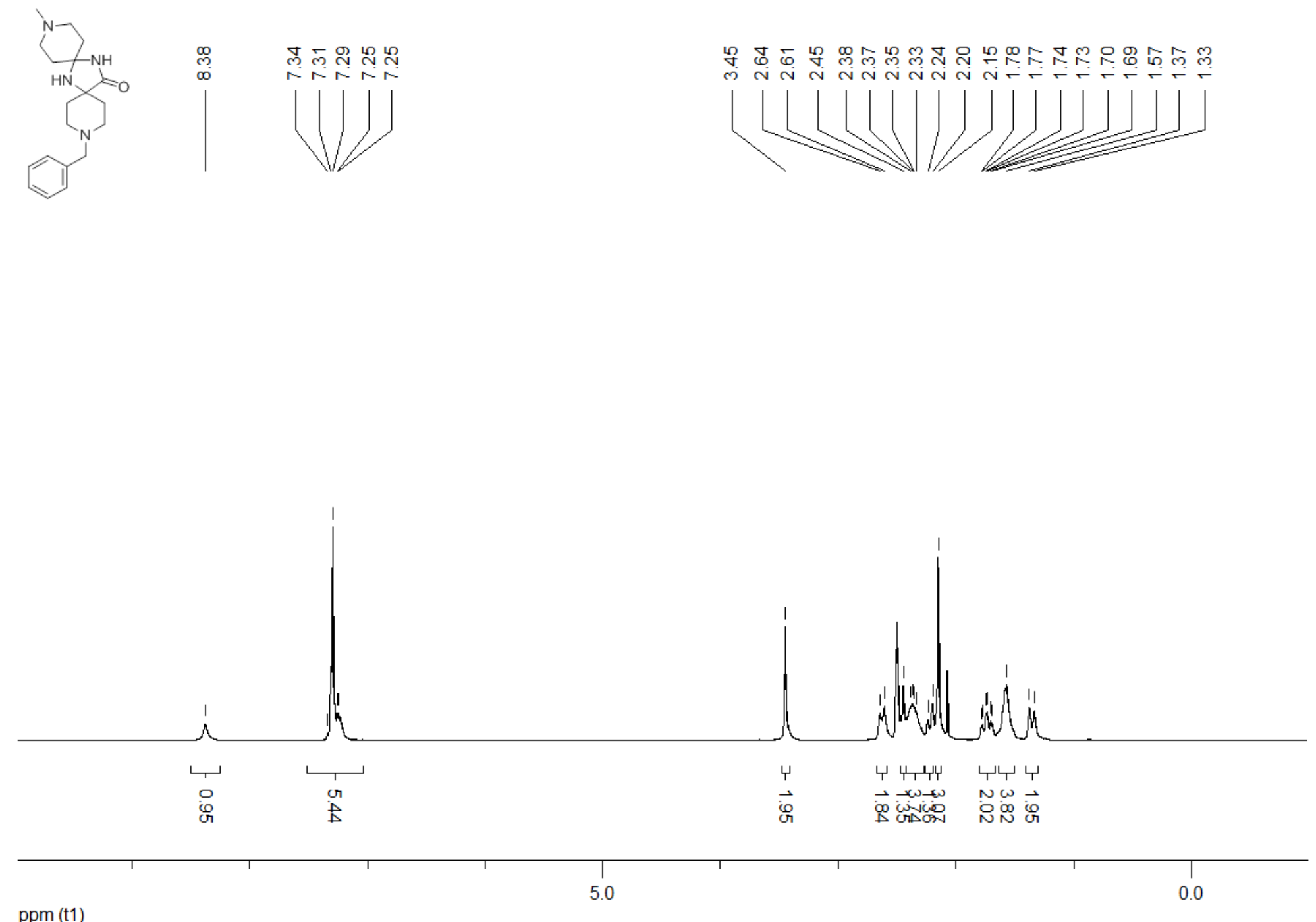
${ }^{13}$ C NMR (100 MHz, DMSO- $\left.d_{6}\right)$ 3-benzyl-11-methyl-3,7,11,14-tetraazadispiro[5.1.5 $\left.{ }^{8} .2^{6}\right]$ pentadecan-15-one $4\{6\}$
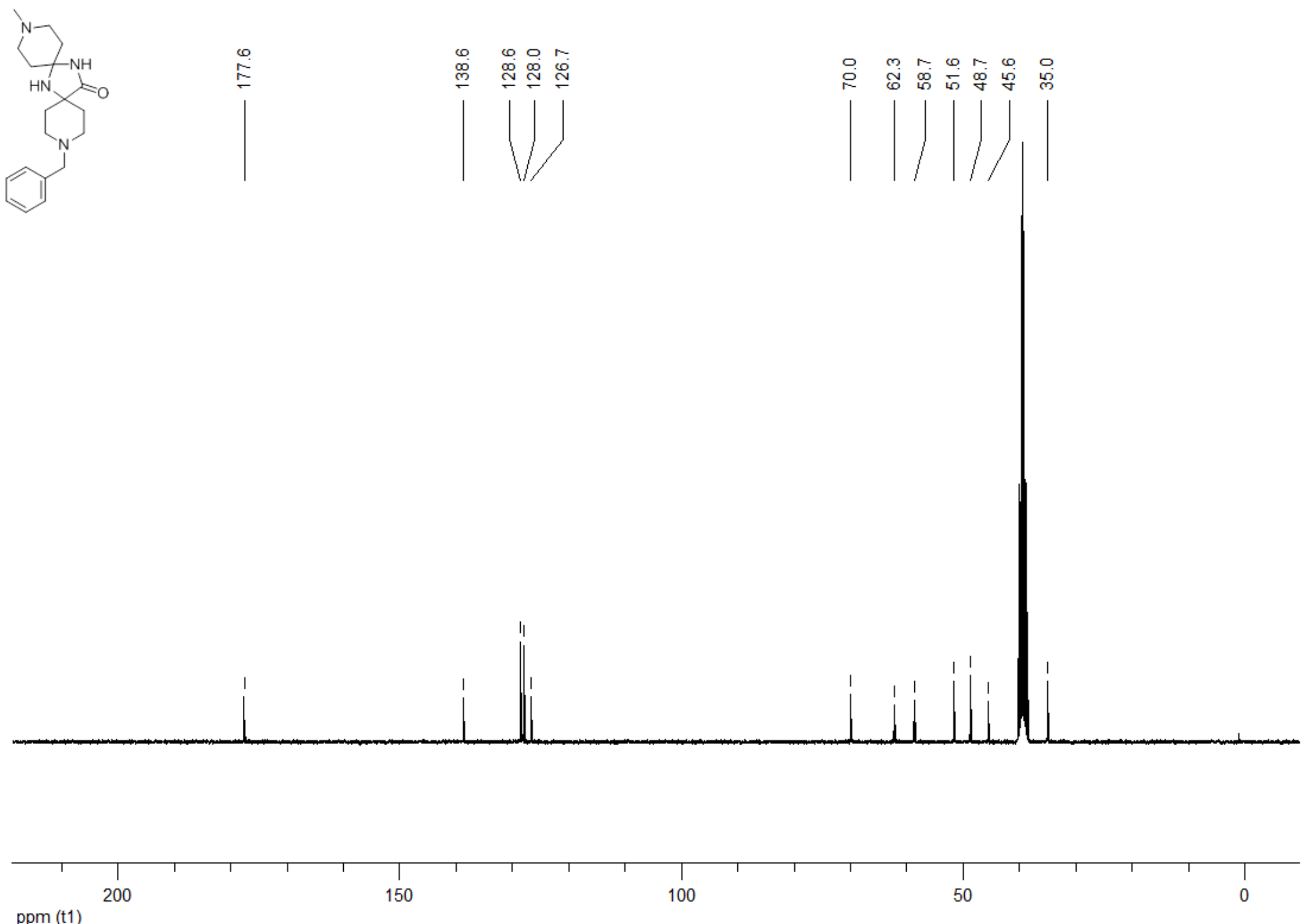
${ }^{1}$ H NMR (300 MHz, CDCl 3 ) 3-benzyl-3,7,15-triazadispiro[5.1.6 $\left.{ }^{8} .2^{6}\right]$ hexadecane-16-one 4\{7\}
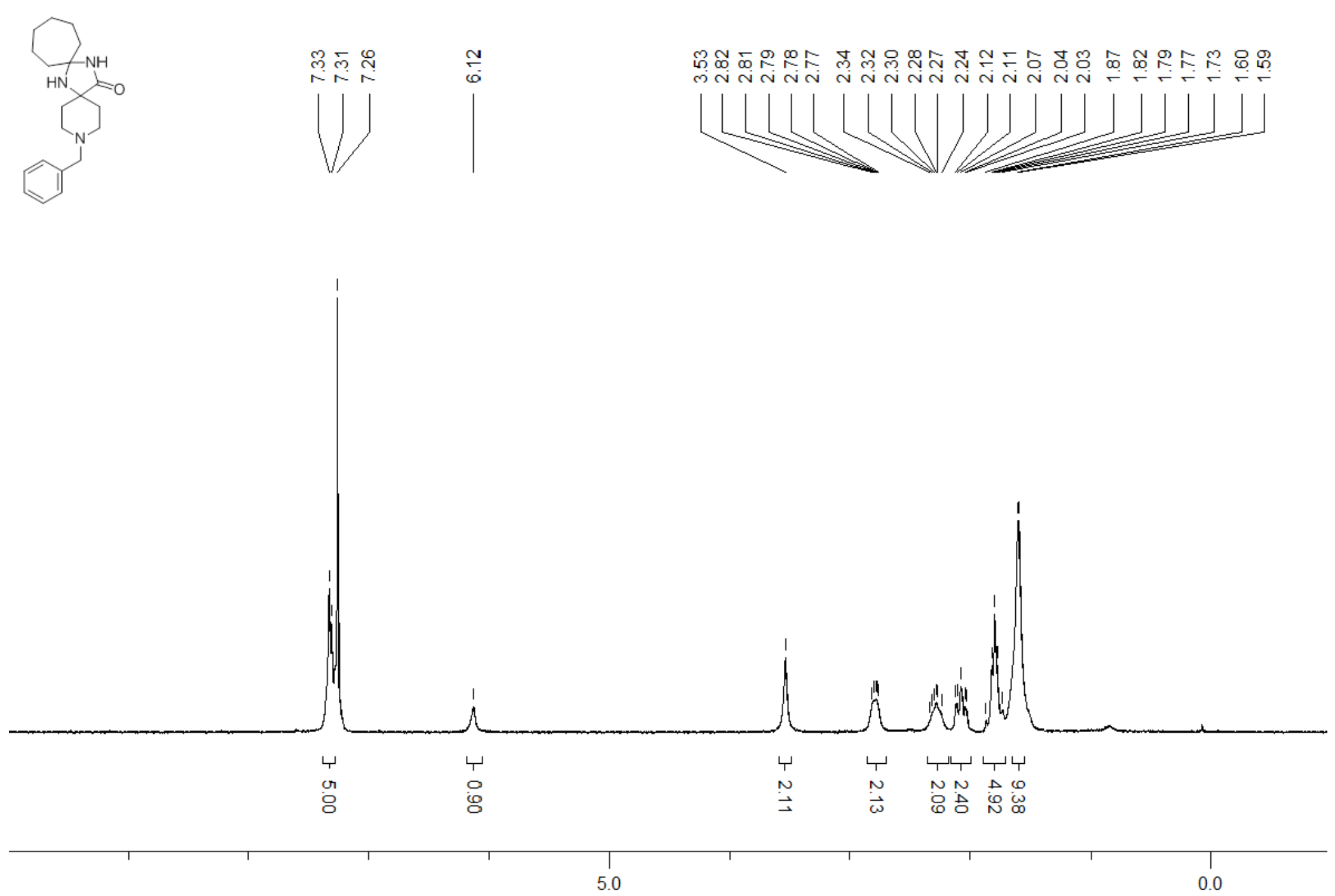

ppm (t1) 
${ }^{1}$ H NMR (300 MHz, DMSO-d $d_{6}$ 1-acetyl-8-benzyl-2,2-dimethyl-1,3,8-triazaspiro[4.5]decan-4-one 5\{1\}

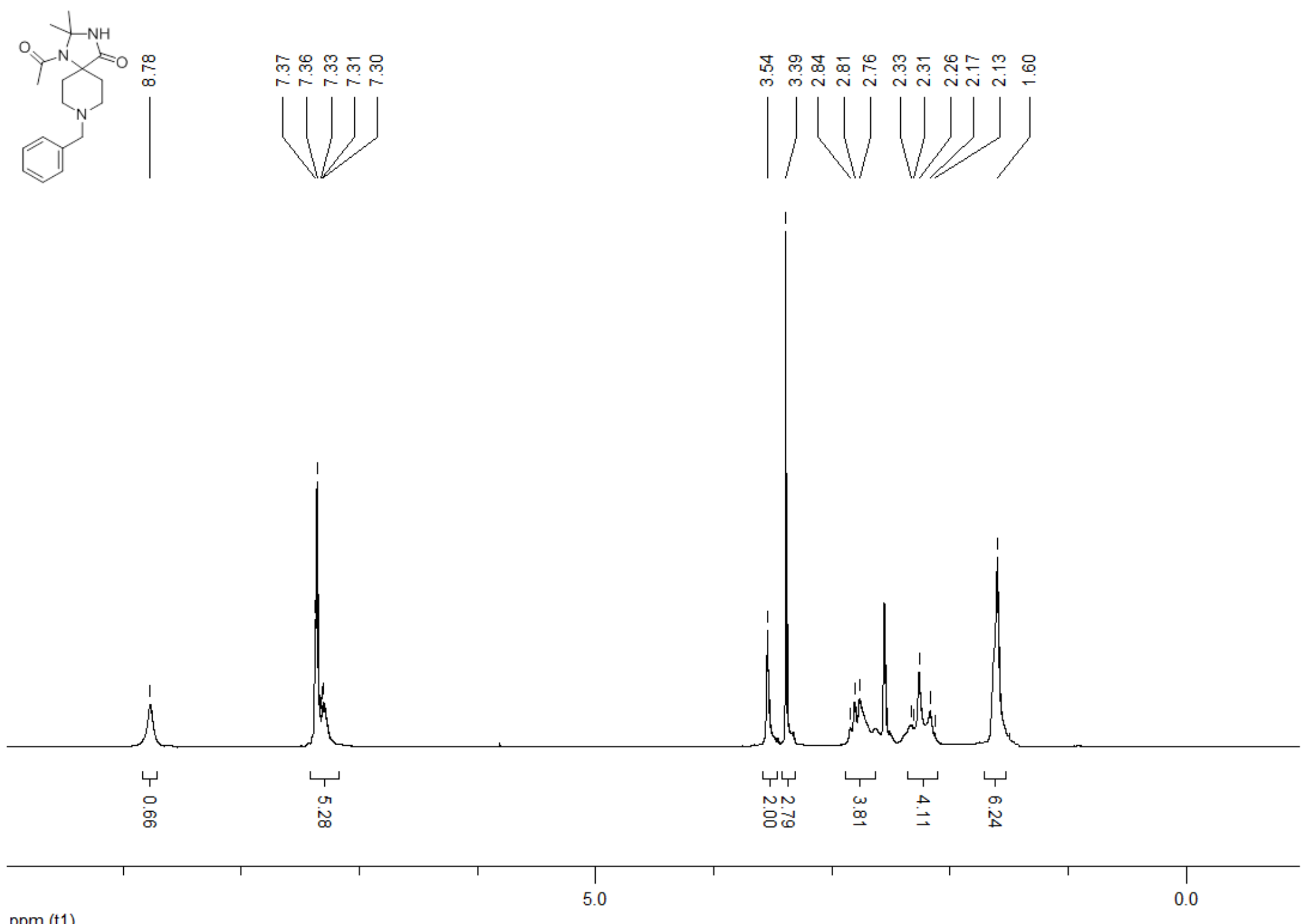

ppm (t1) 
HPLC-MS (ESI) 1-acetyl-8-benzyl-2,2-dimethyl-1,3,8-triazaspiro[4.5]decan-4-one 5\{1\}

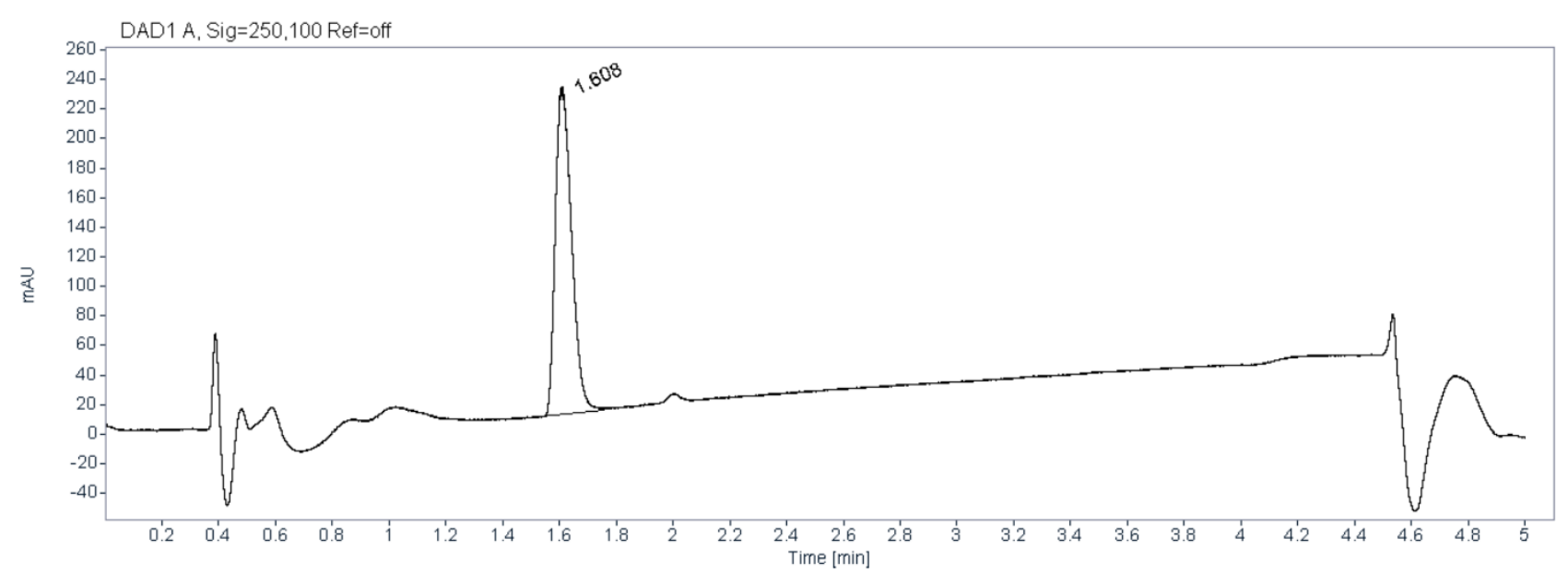

MSD1 SPC, MM-ES+APCI

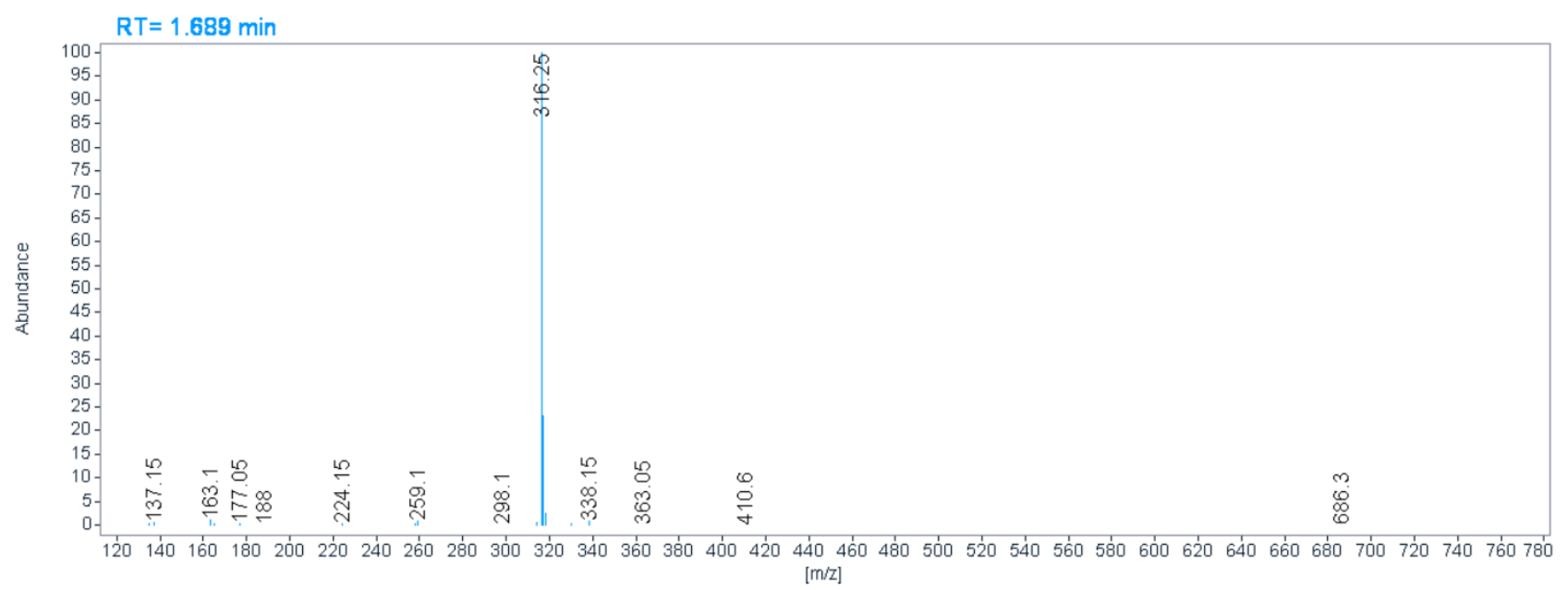


${ }^{1} \mathrm{H}$ NMR (300 MHz, $\mathrm{CDCl}_{3}$ ) 6-acetyl-10-benzyl-6,10,14-triazadispiro[4.1.5 $\left.{ }^{7} .2^{5}\right]$ tetradecan-13-one 5\{3\}

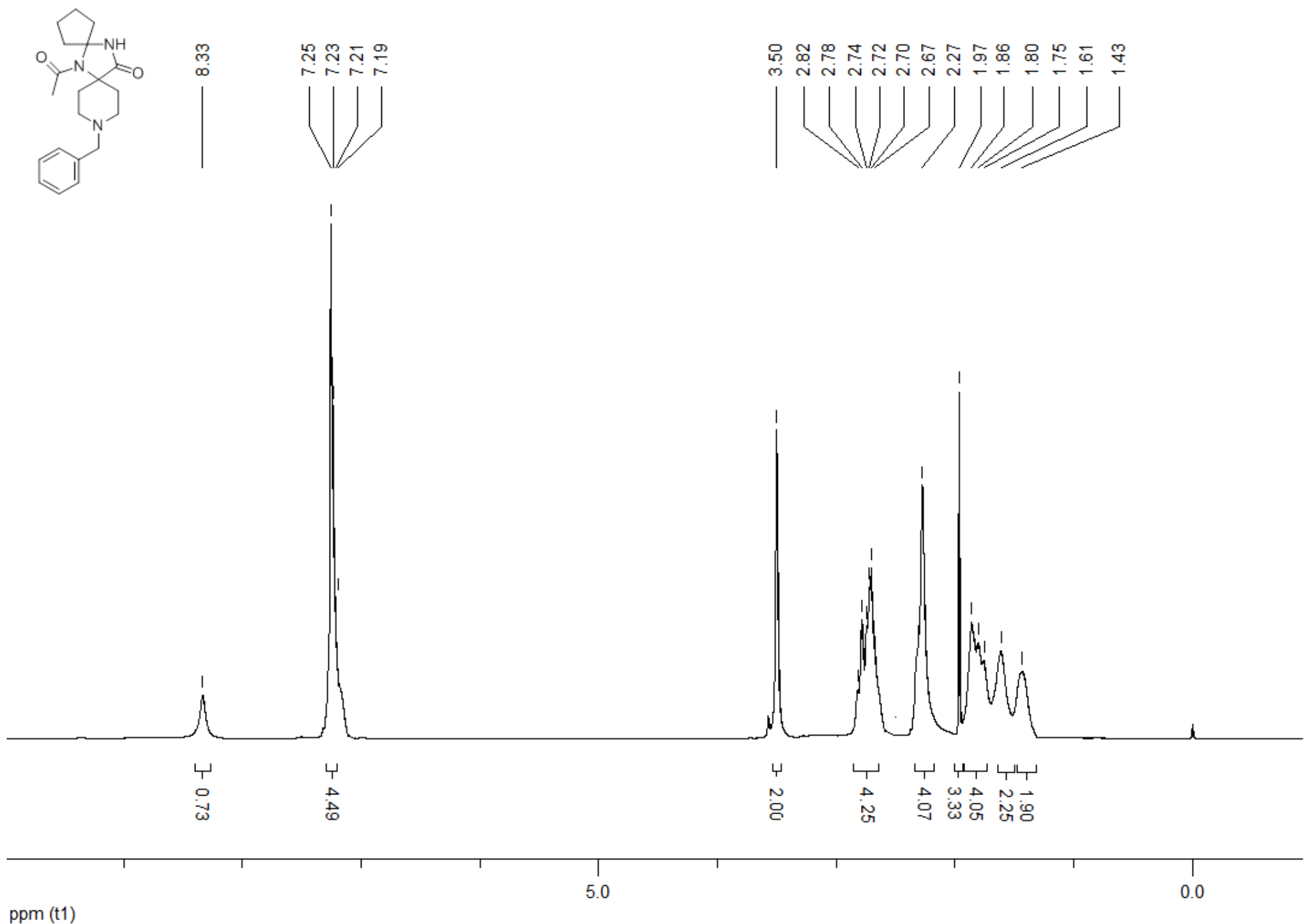


${ }^{1} \mathrm{H}$ NMR (300 MHz, CDCl ${ }_{3}$ ) 7-acetyl-3-benzyl-3,7,14-triazadispiro[5.1.5 ${ }^{8} .2^{6}$ ]pentadecan-15-one 5\{4\}
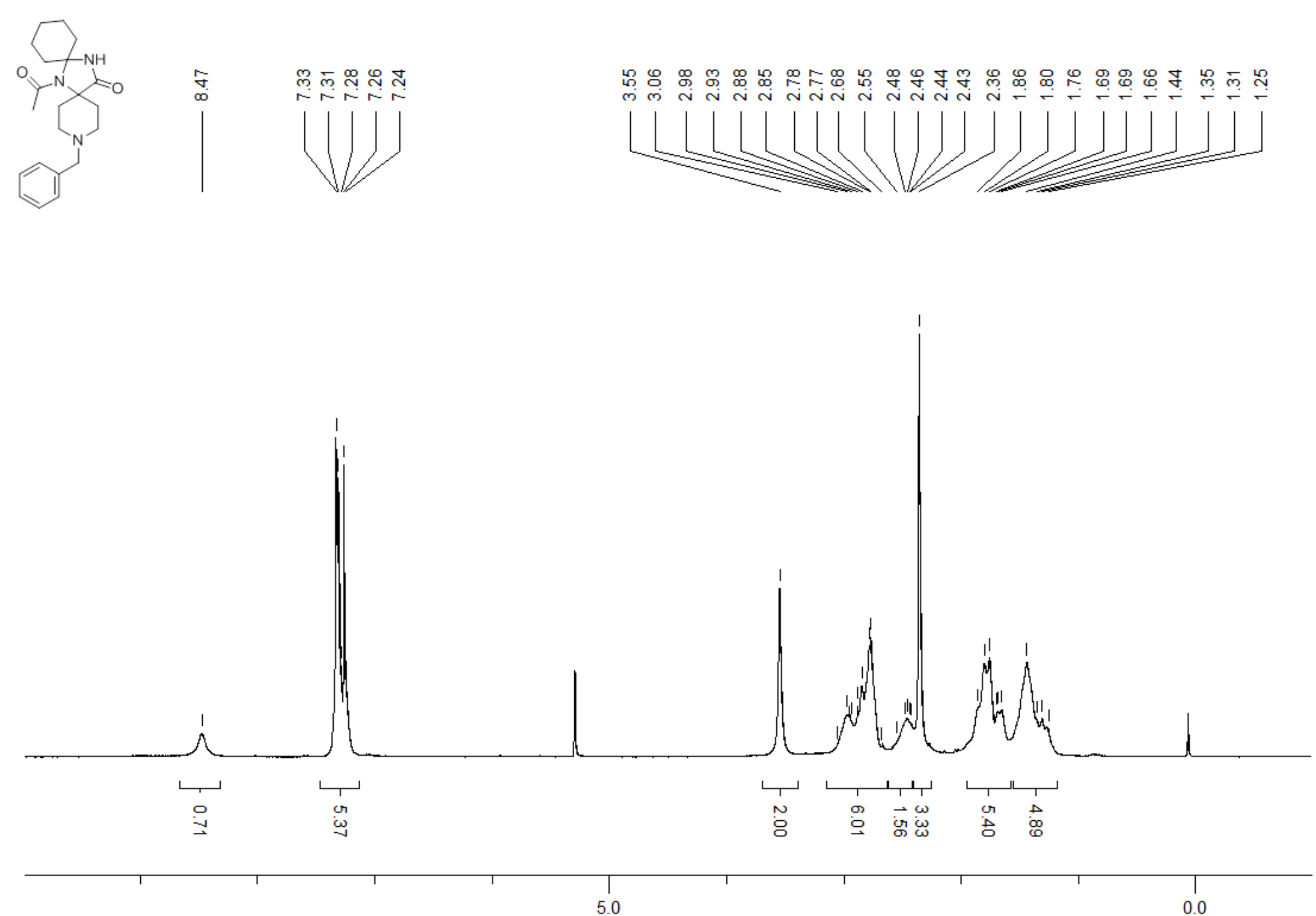

ppm (t1) 
HPLC-MS (ESI) 7-acetyl-3-benzyl-3,7,14-triazadispiro[5.1.5 $\left.{ }^{8} .2^{6}\right]$ pentadecan-15-one $5\{4\}$

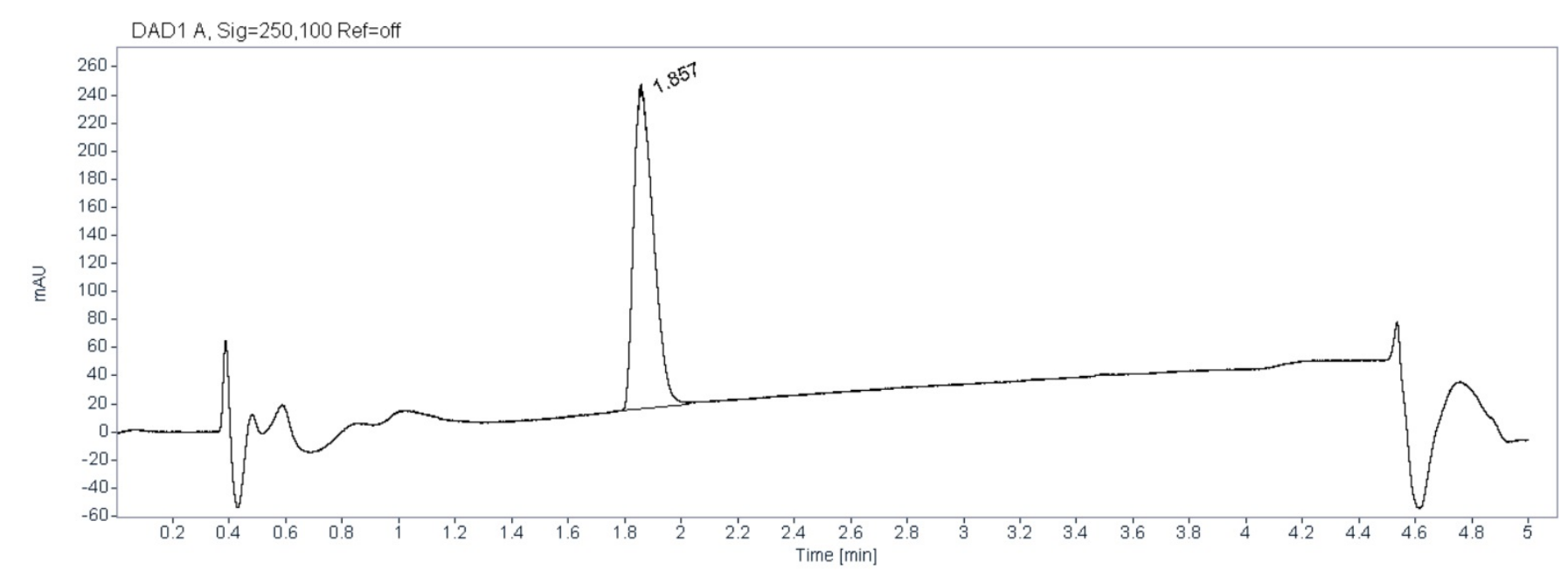

MSD1 SPC, MM-ES+APCI

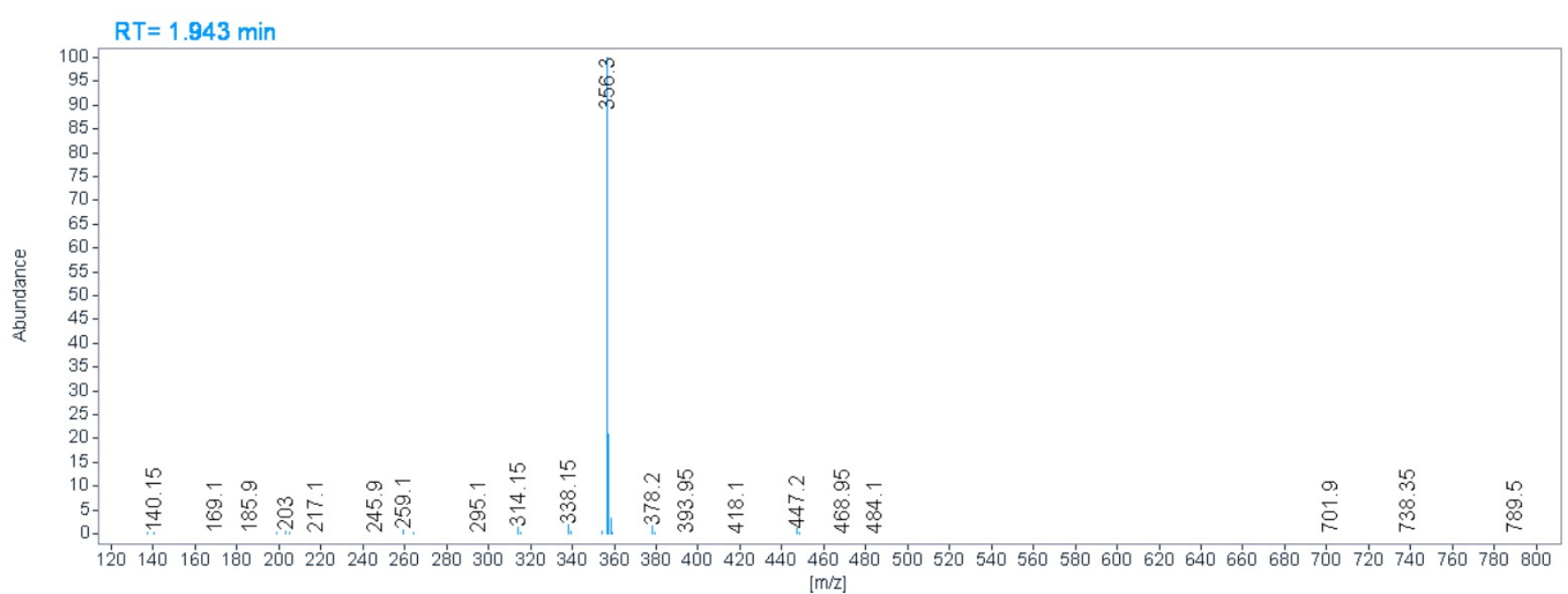


${ }^{1}$ H NMR (300 MHz, DMSO- $d_{6}$ ) 7-acetyl-3-benzyl-11-oxa-3,7,14-triazadispiro[5.1.5 ${ }^{8} .{ }^{6}$ ]pentadecan-15-one 5\{5\}

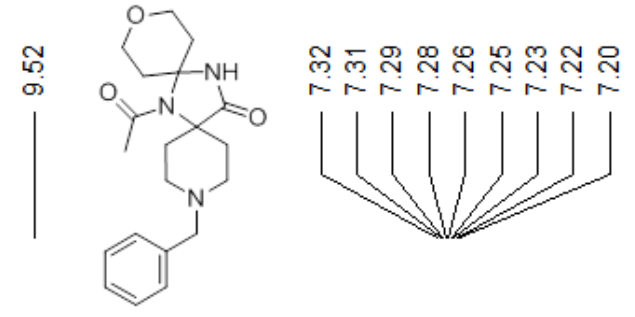

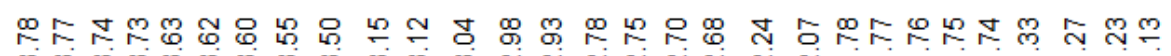
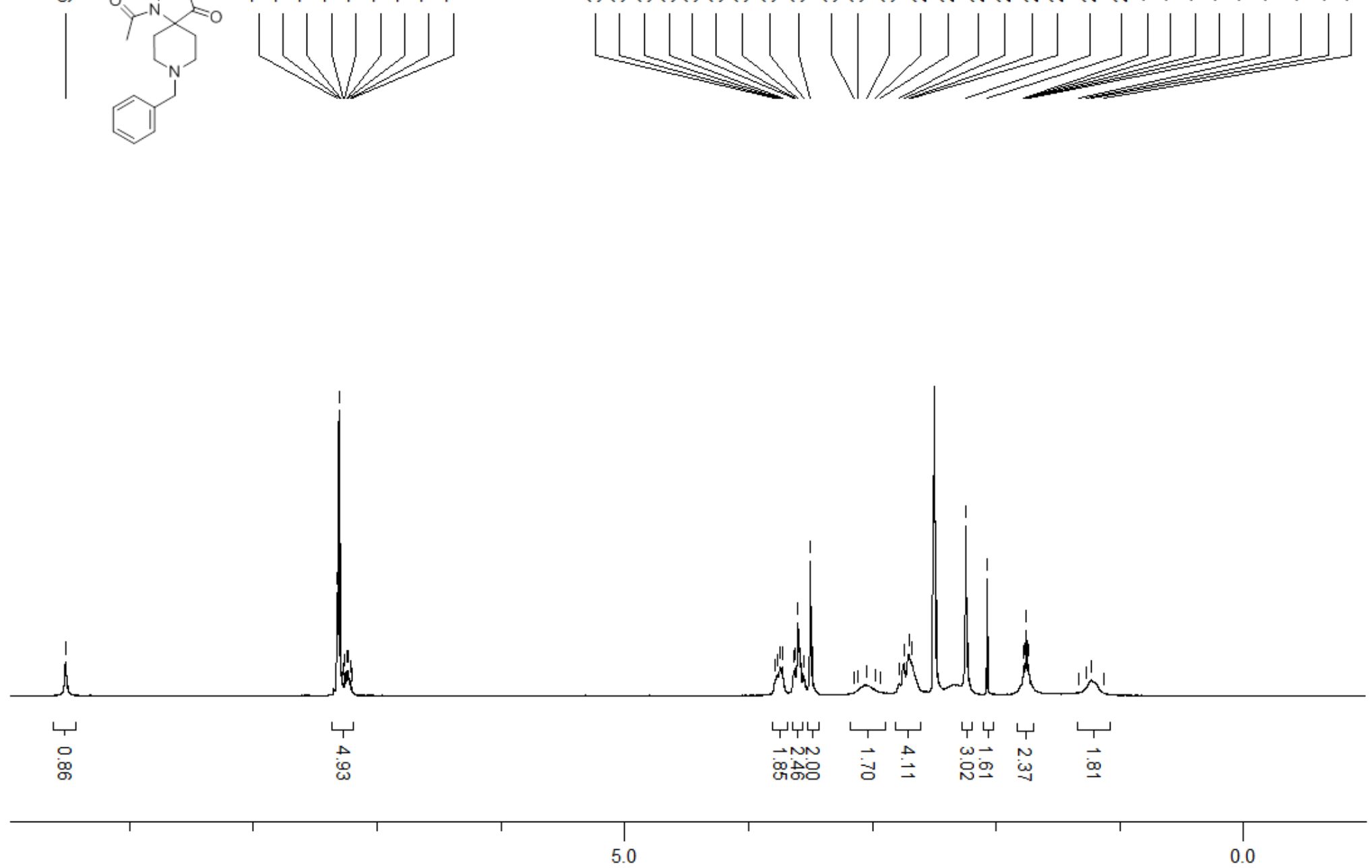

ppm (t1) 
HPLC-MS (ESI) 7-acetyl-3-benzyl-11-oxa-3,7,14-triazadispiro[5.1.5 $\left.{ }^{8} .2^{6}\right]$ pentadecan-15-one 5\{5\}

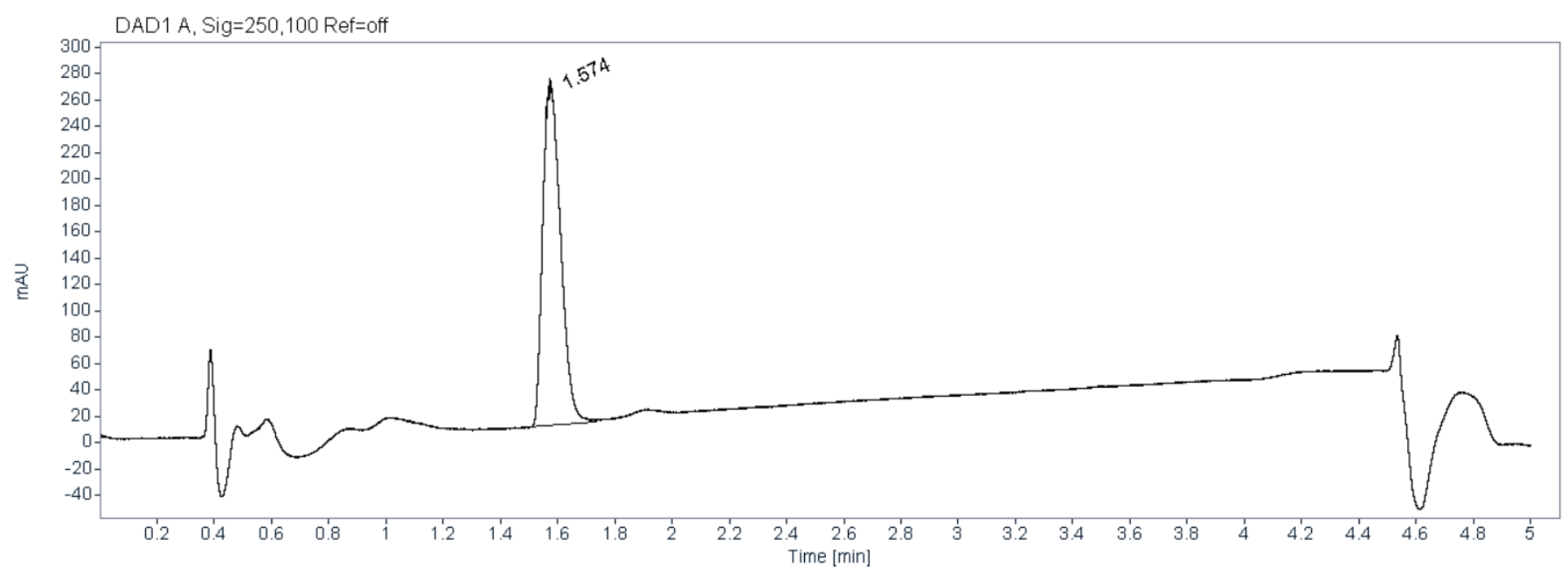

MSD1 SPC, MM-ES+APCI

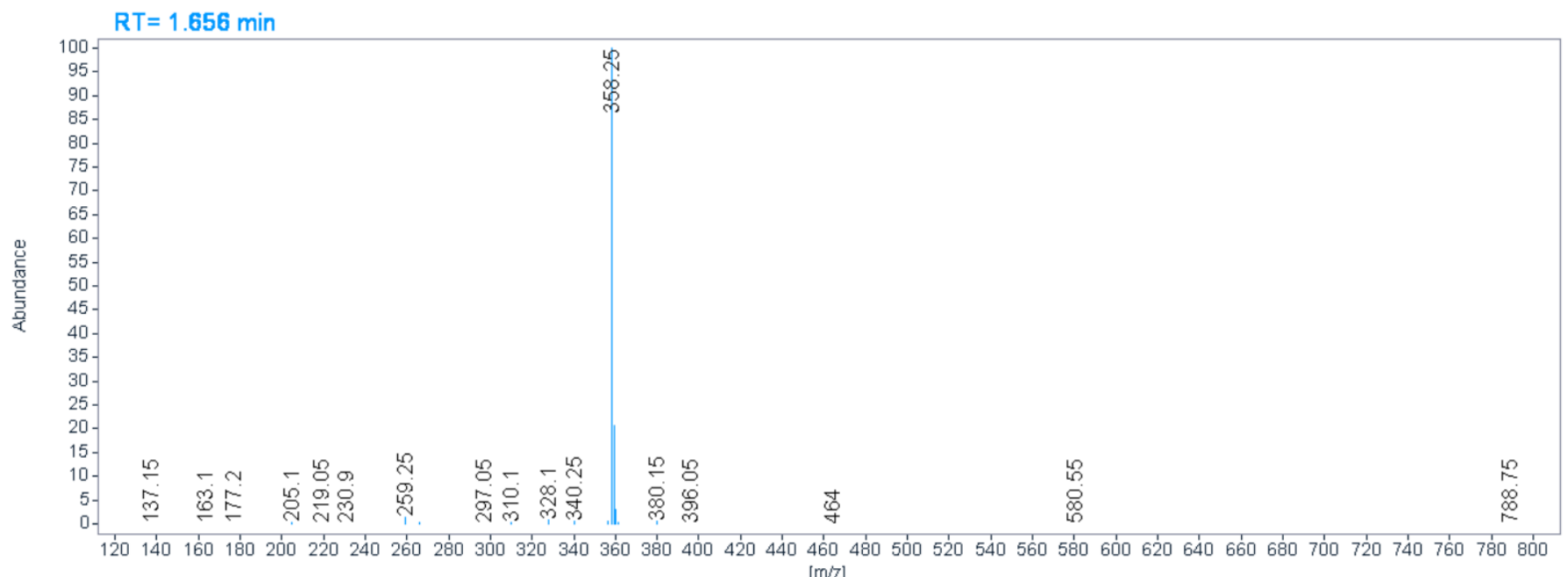


${ }^{1}$ H NMR (300 MHz, DMSO- $d_{6}$ ) 7-acetyl-3-benzyl-11-methyl-3,7,11,14-tetraazadispiro[5.1.5.$^{8} .2^{6}$ ]pentadecan-15-one 5\{6\}

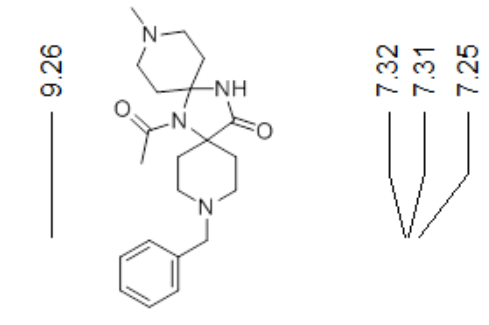

员
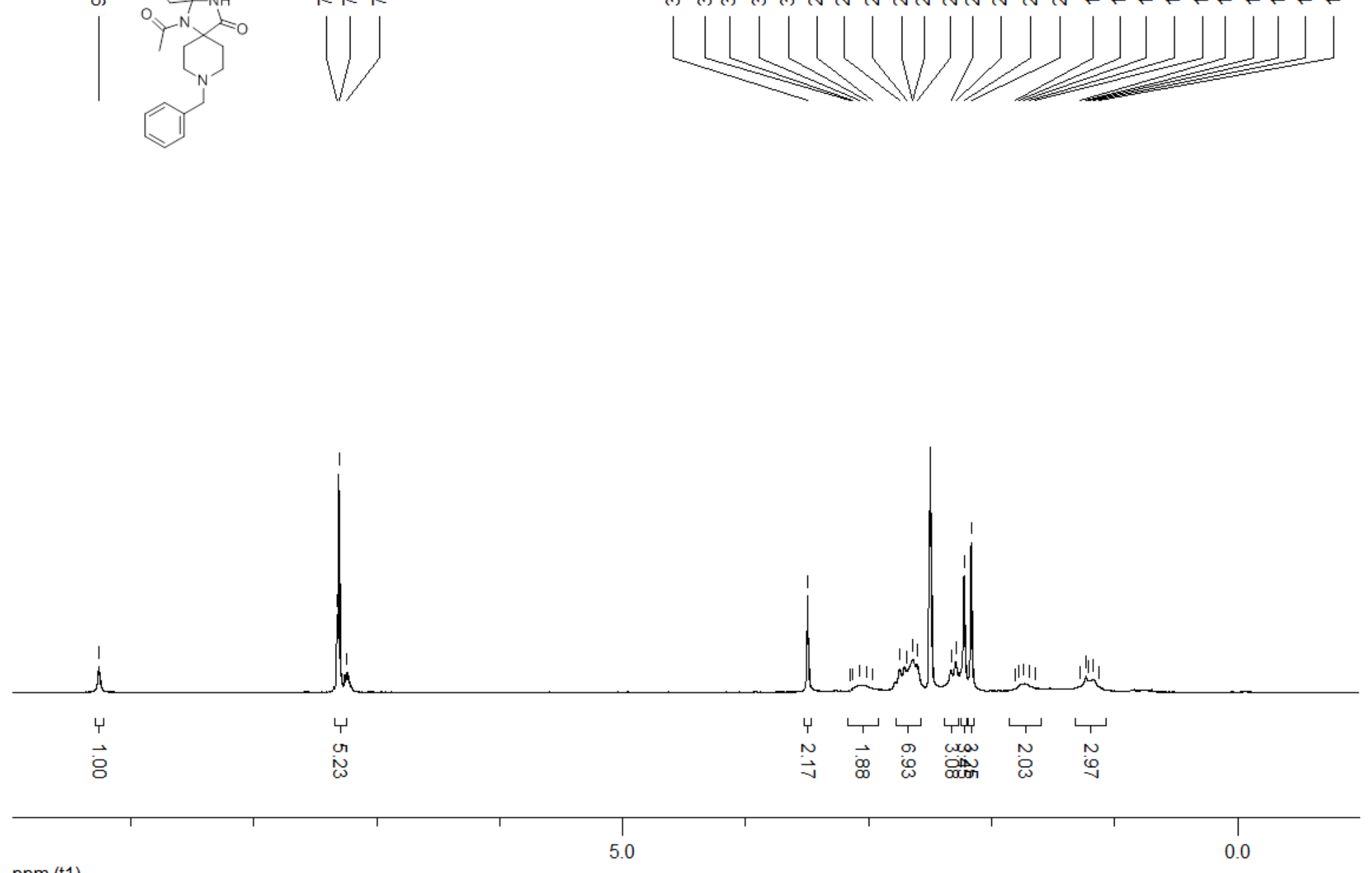

ppm (t1) 
${ }^{1} \mathrm{H}$ NMR (300 MHz, $\mathrm{CDCl}_{3}$ ) 1-benzoyl-8-benzyl-2,2-dimethyl-1,3,8-triazaspiro[4.5]decan-4-one \{5\}9
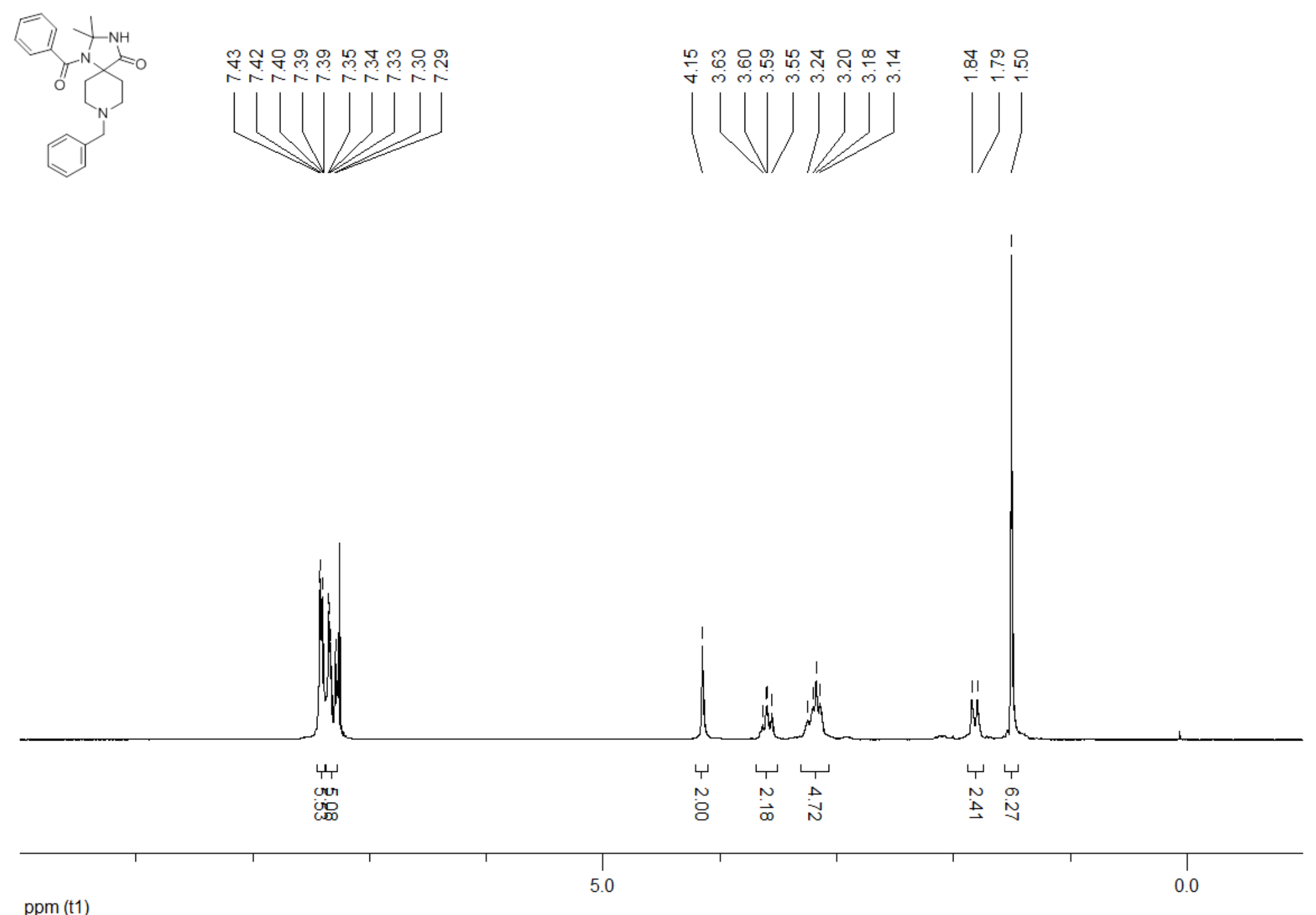
${ }^{1}$ H NMR (300 MHz, $\mathrm{CDCl}_{3}$ ) 3-benzyl-7-propanoyl-3,7,14-triazadispiro[5.1.5 $\left.{ }^{8} 2^{6}\right]$ pentadecan-15-one 5\{10\}
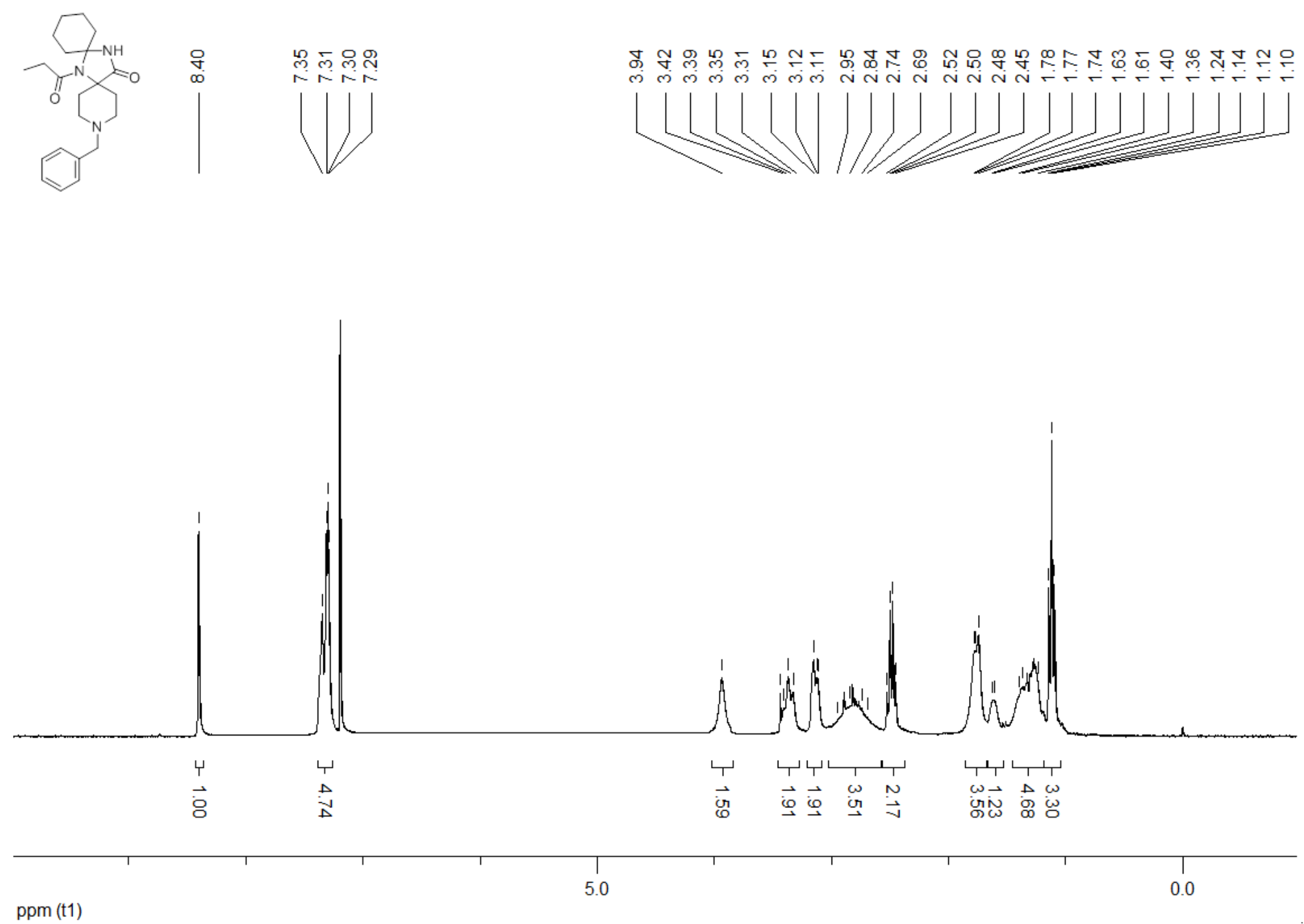
${ }^{1}$ H NMR (300 MHz, DMSO- $d_{6}$ ) 6-acetyl-6,10,14-triazadispiro $\left[4.1 .5^{7} .2^{5}\right]$ tetradecan-13-one $6\{3\}$
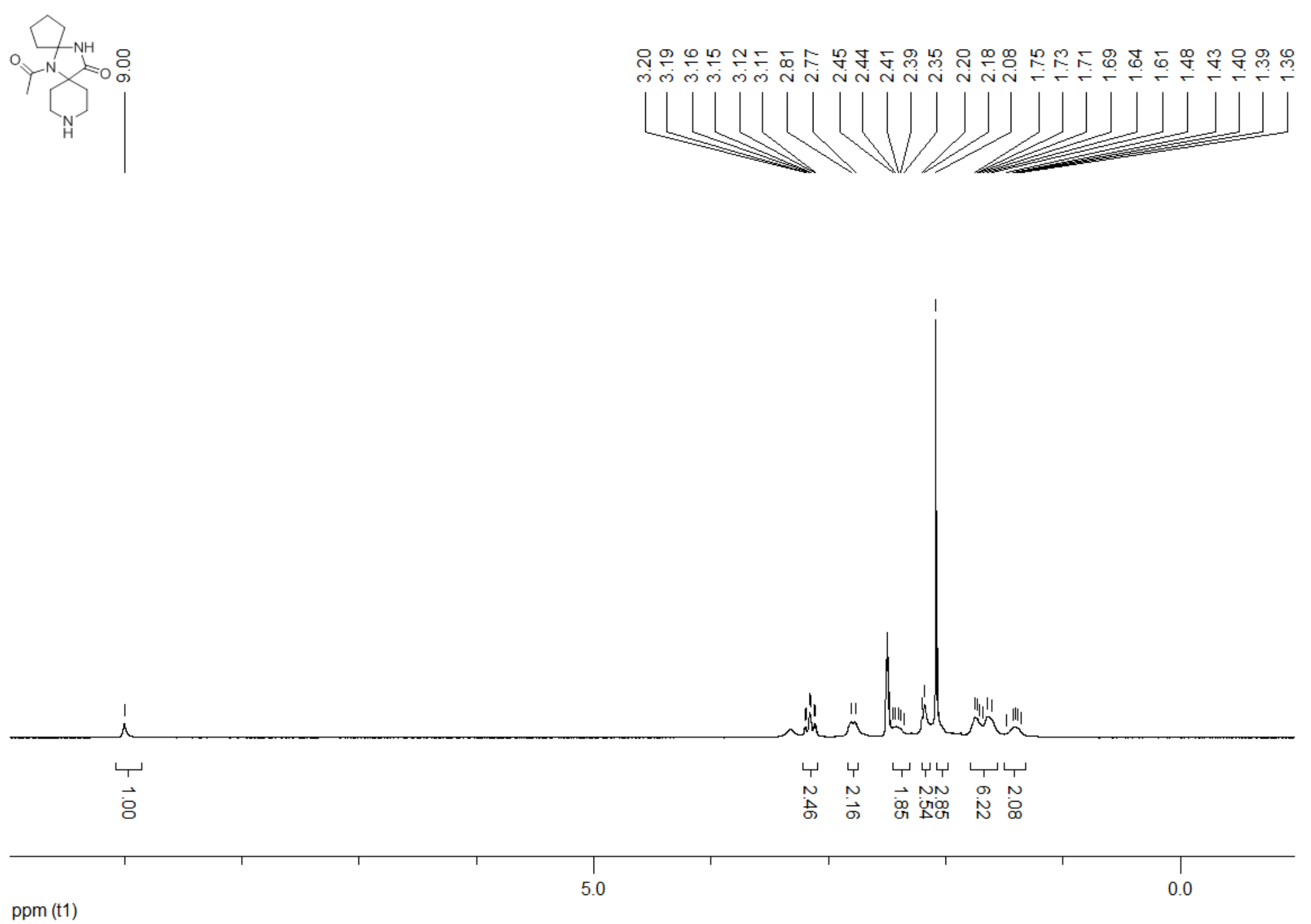
${ }^{1}$ H NMR (300 MHz, DMSO- $d_{6}$ ) 7-acetyl-3,7,14-triazadispiro[5.1.5.$\left.^{8} .2^{6}\right]$ pentadecan-15-one $6\{4\}$

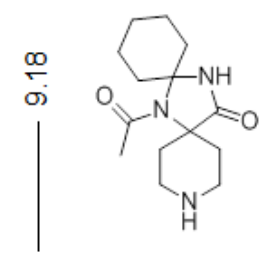

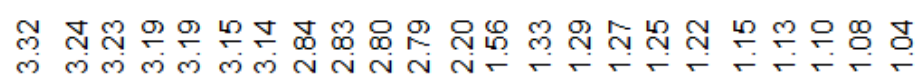
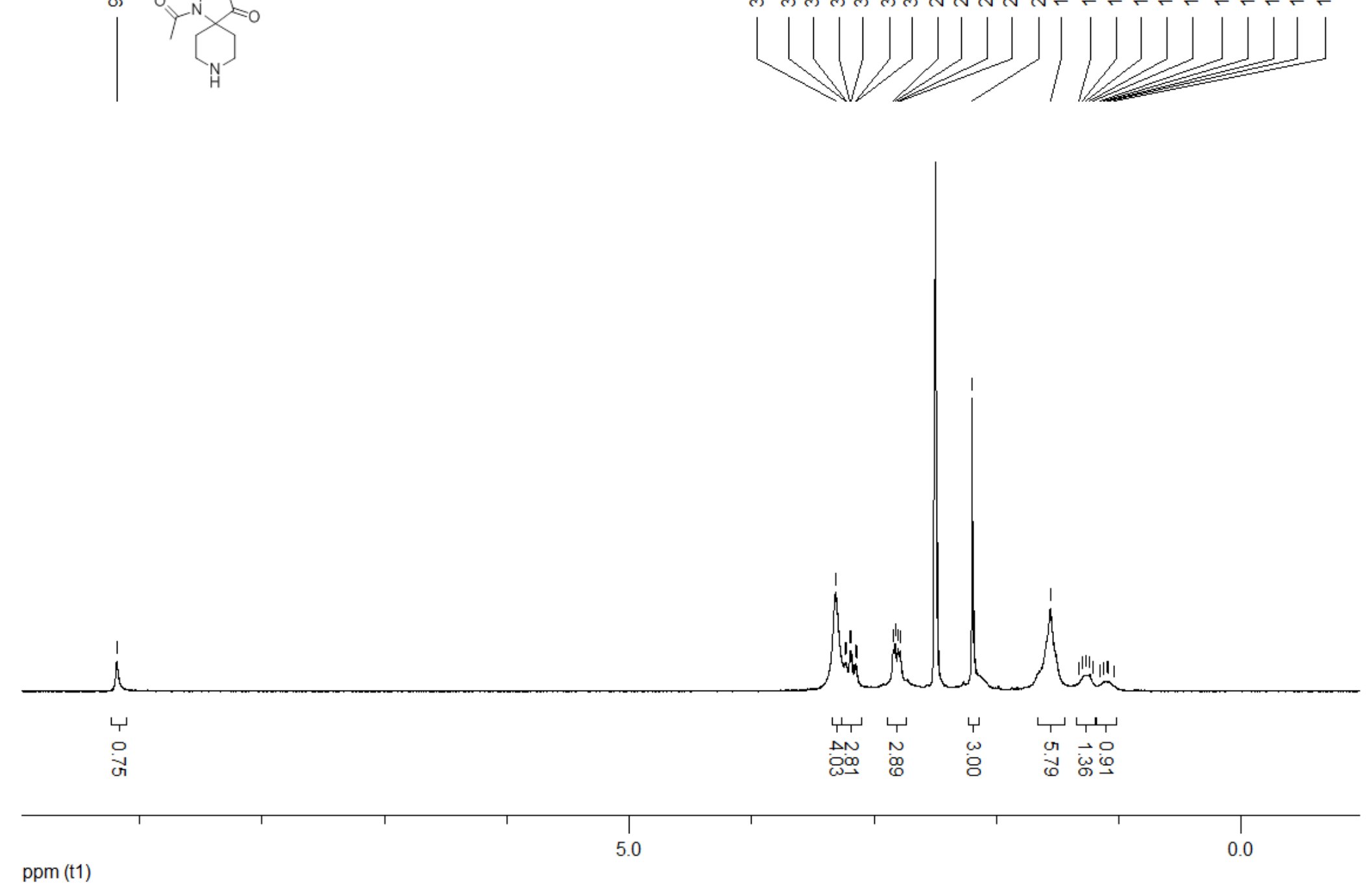
HPLC-MS (ESI) 7-acetyl-3,7,14-triazadispiro[5.1.5 $\left.{ }^{8} .2^{6}\right]$ pentadecan-15-one $6\{4\}$

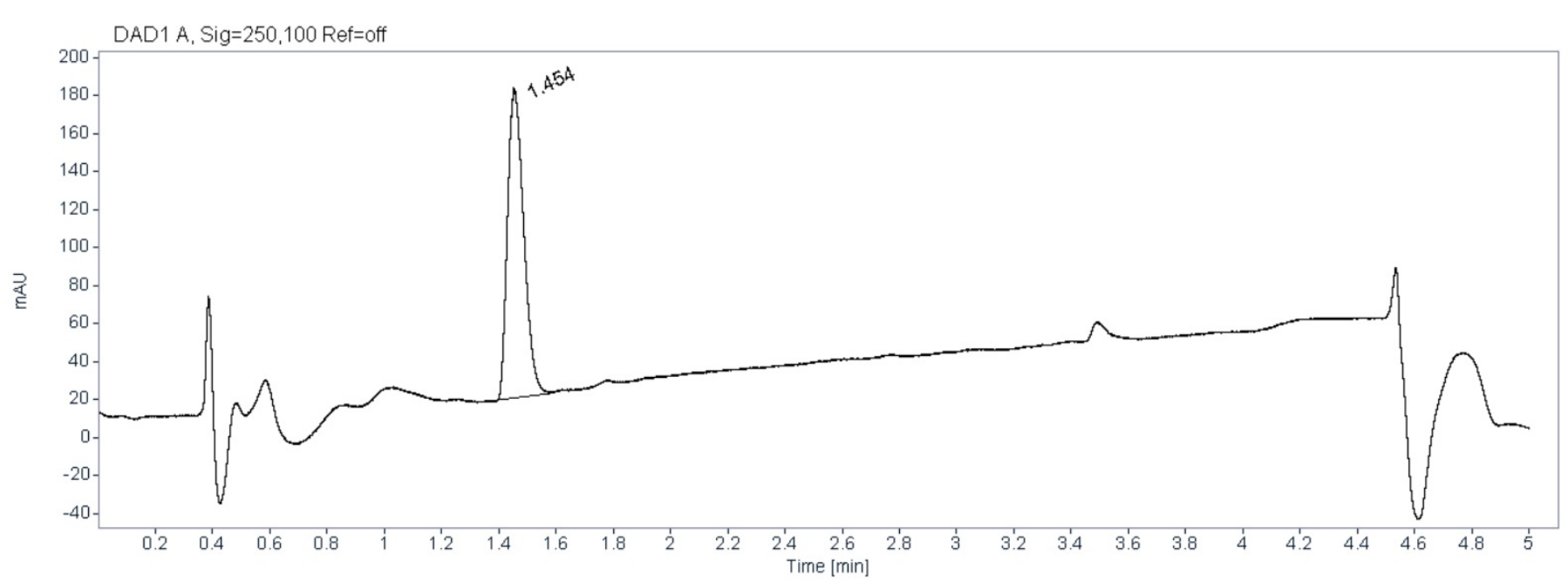

MSD1 SPC, MM-ES+APCI

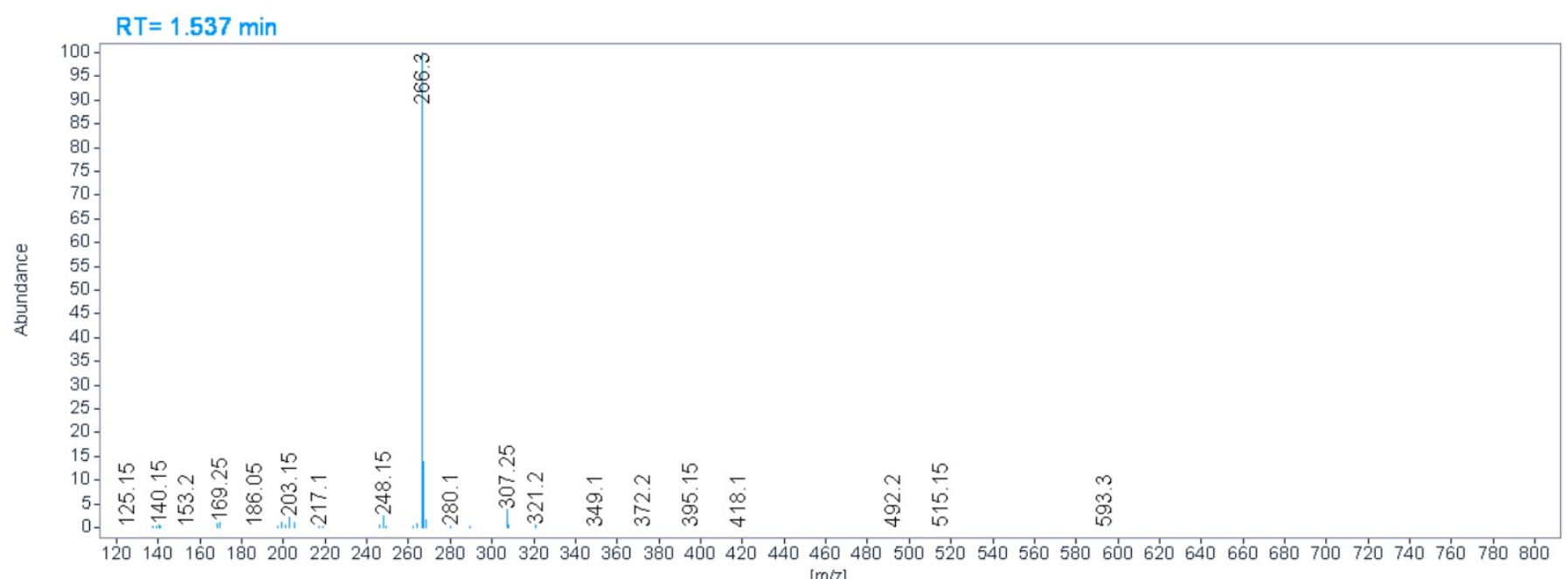


${ }^{1}$ H NMR (300 MHz, DMSO-d $d_{6}$ ) 7-acetyl-11-methyl-3,7,11,14-tetraazadispiro[5.1.5.$^{8}{ }^{6}$ ]pentadecan-15-one $6\{6\}$

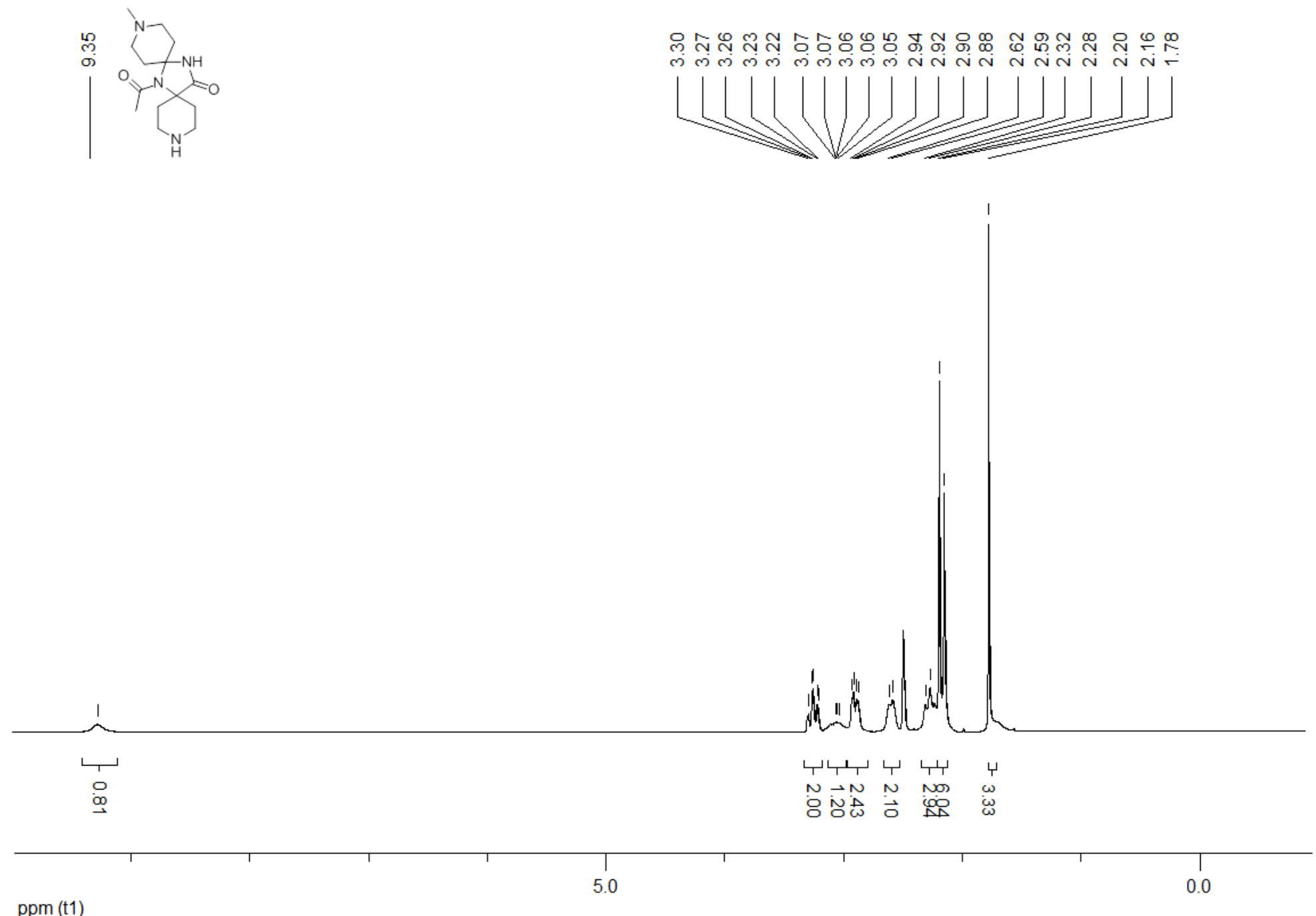


${ }^{1}$ H NMR (300 MHz, DMSO-d $d_{6}$ 1-benzoyl-2,2-dimethyl-1,3,8-triazaspiro[4.5]decan-4-one 6\{9\}
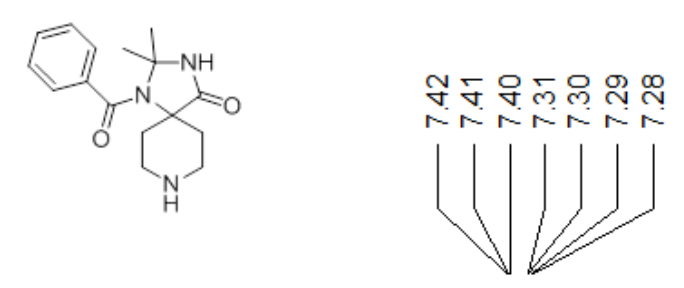

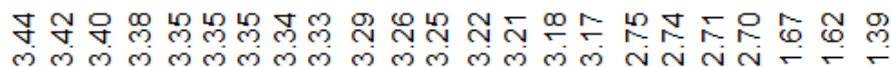
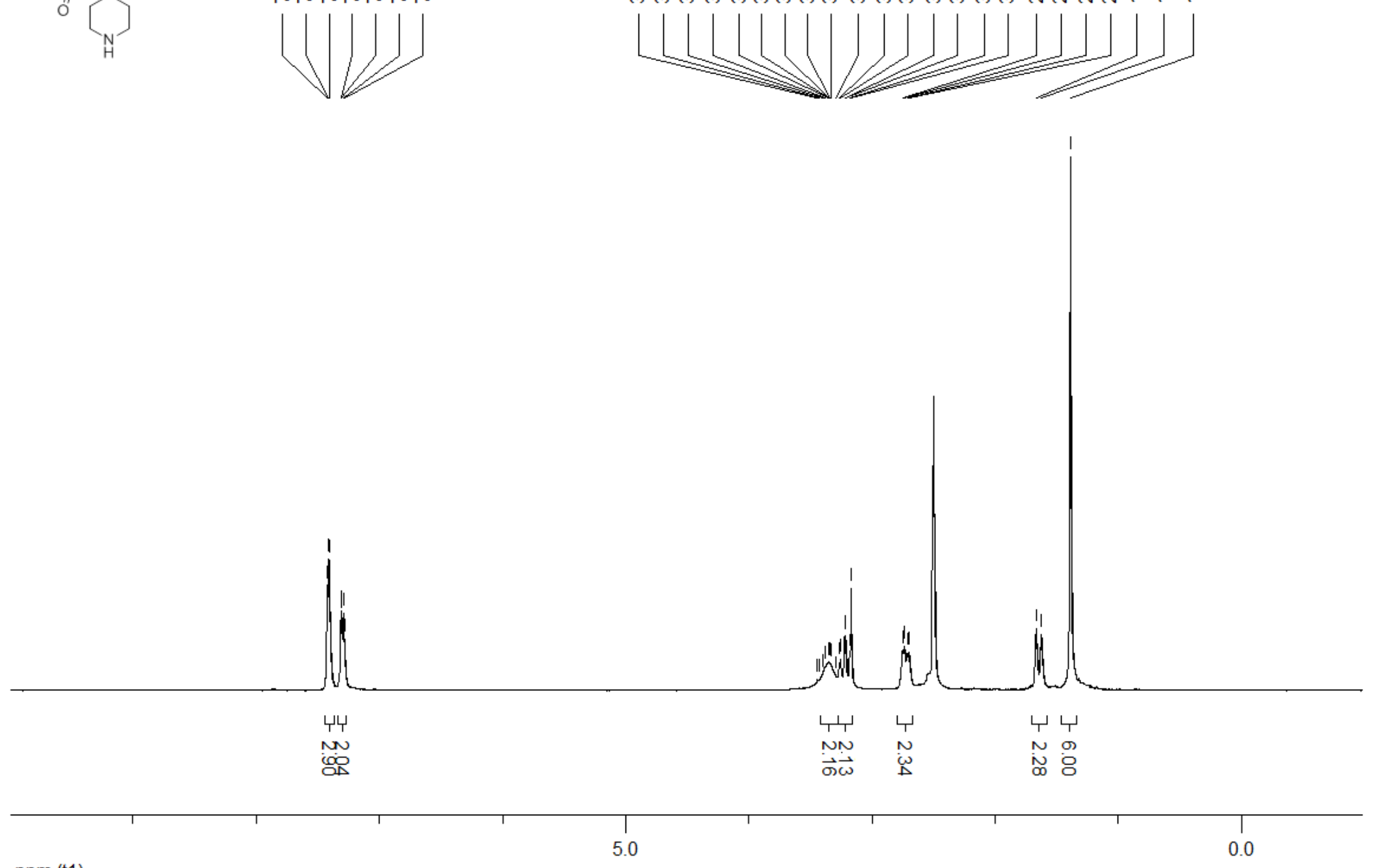

ppm (t1) 


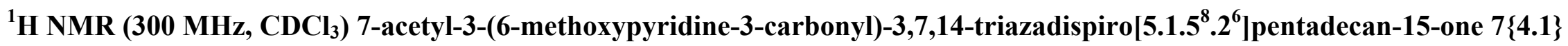
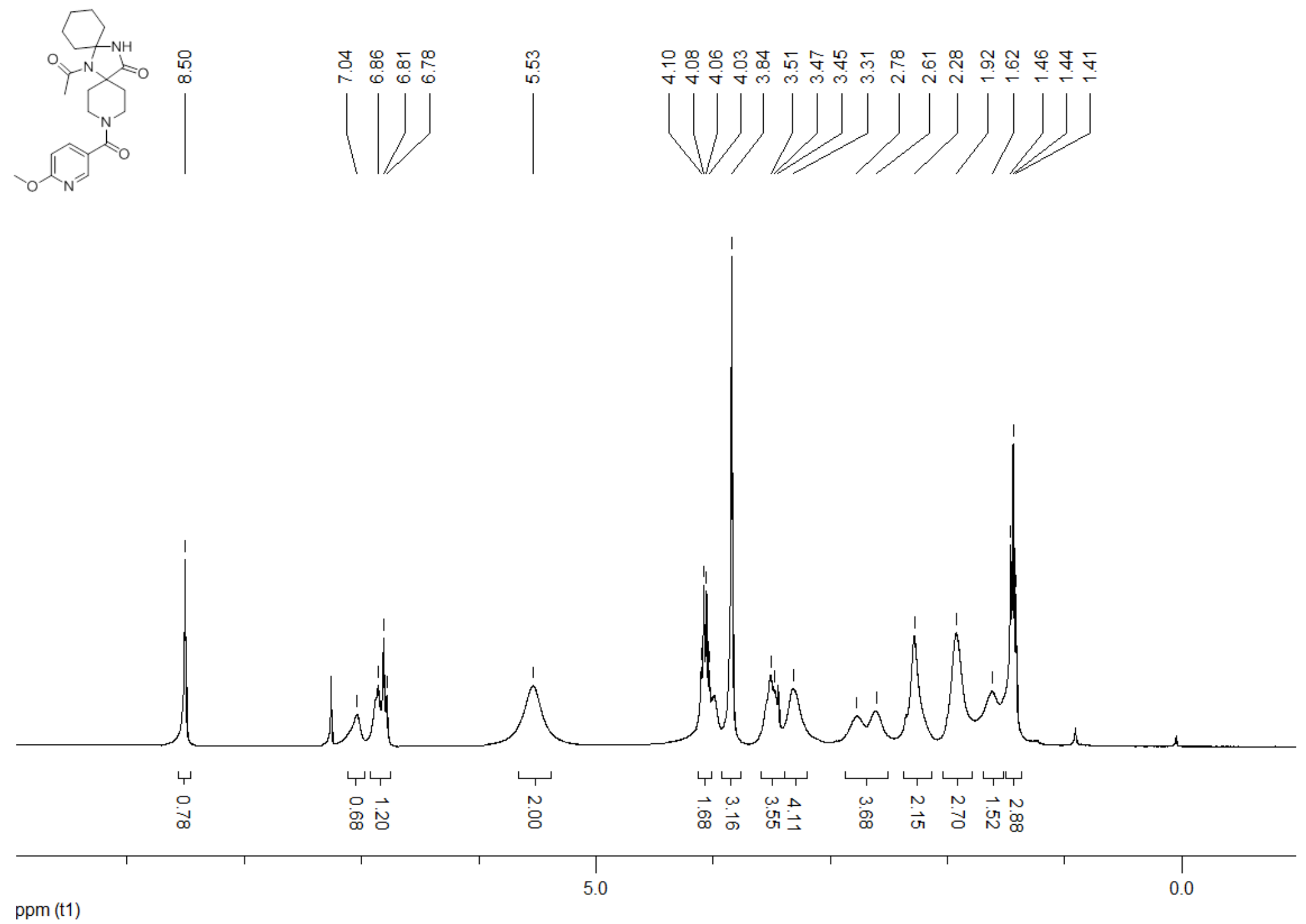
${ }^{1} \mathrm{H}$ NMR (300 MHz, CDCl 3$)$ 7-acetyl-3-(2,2-dimethylbutanoyl)-11-oxa-3,7,14-triazadispiro[5.1.5 ${ }^{8} .^{6}{ }^{6}$ pentadecan-15-one 7\{5.1\}
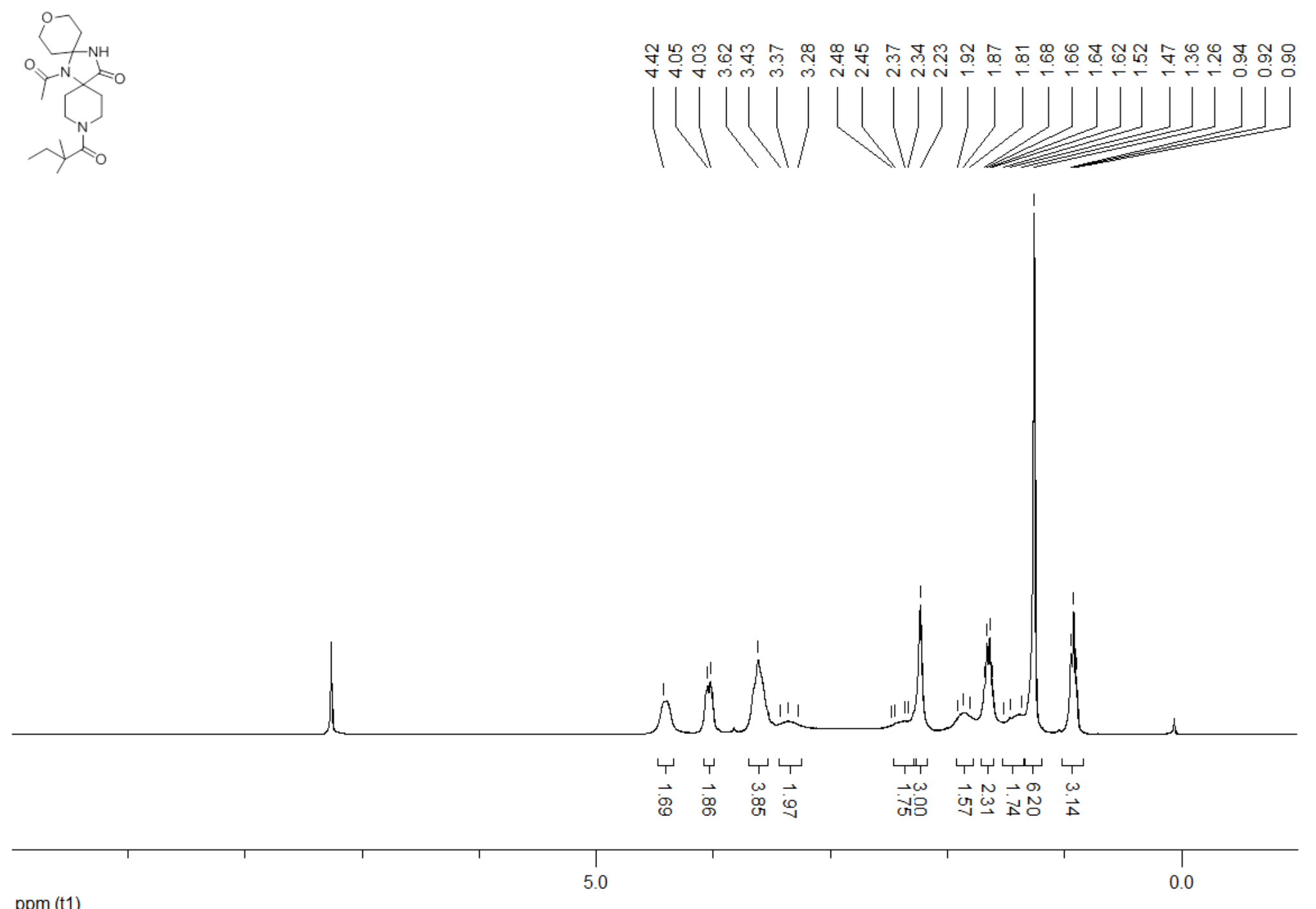

ppm (t1) 
HPLC-MS (ESI) 7-acetyl-3-(2,2-dimethylbutanoyl)-11-oxa-3,7,14-triazadispiro[5.1.5 $\left.{ }^{8} 2^{6}\right]$ pentadecan-15-one $7\{5.1\}$

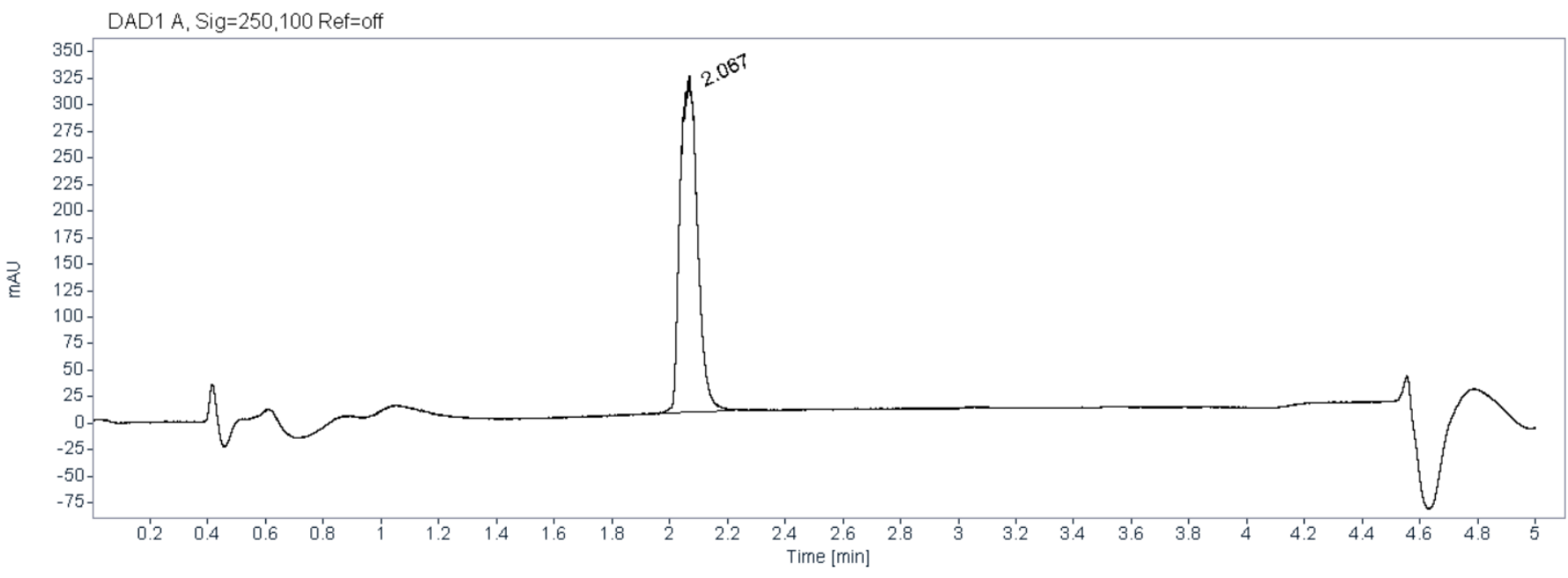

MSD1 SPC, MM-ES+APCI

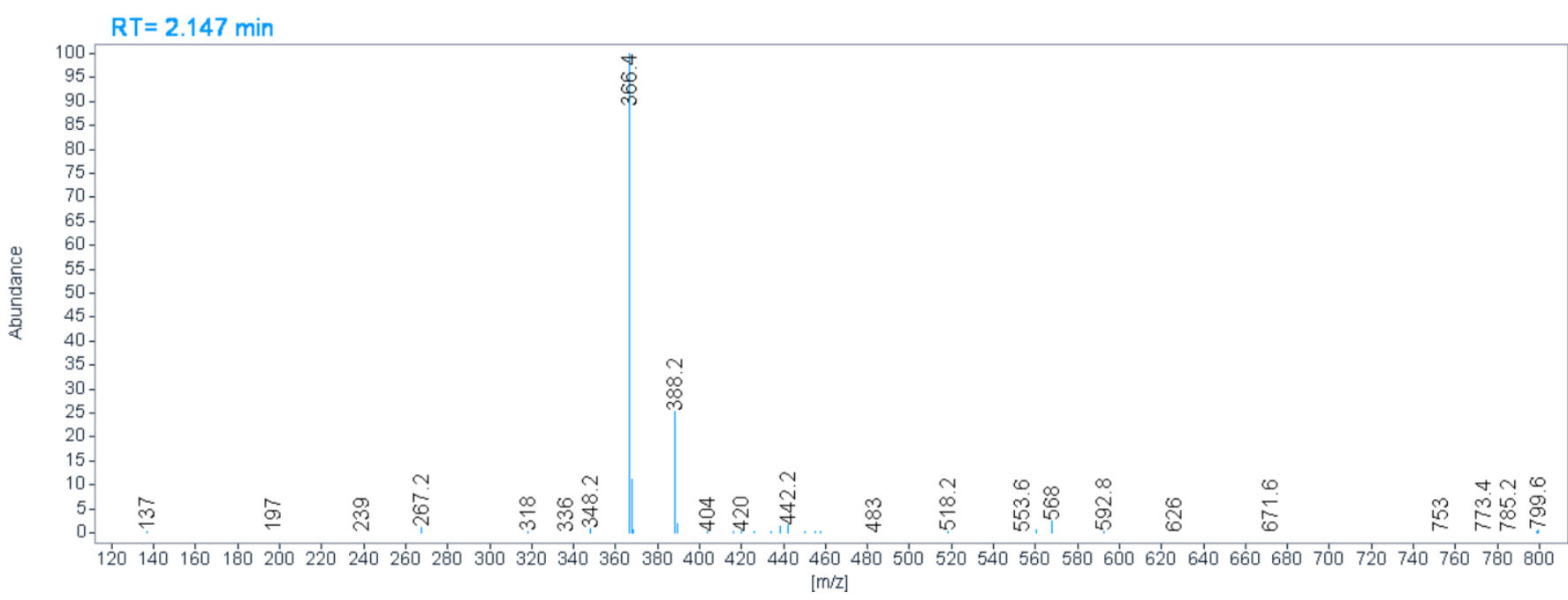


${ }^{1} \mathrm{H}$ NMR (300 MHz, $\mathrm{CDCl}_{3}$ ) 6-acetyl-10-ethyl-6,10,14-triazadispiro[4.1.5 $\left.{ }^{7} .^{5}\right]$ tetradecan-13-one 8 $\{3.1\}$
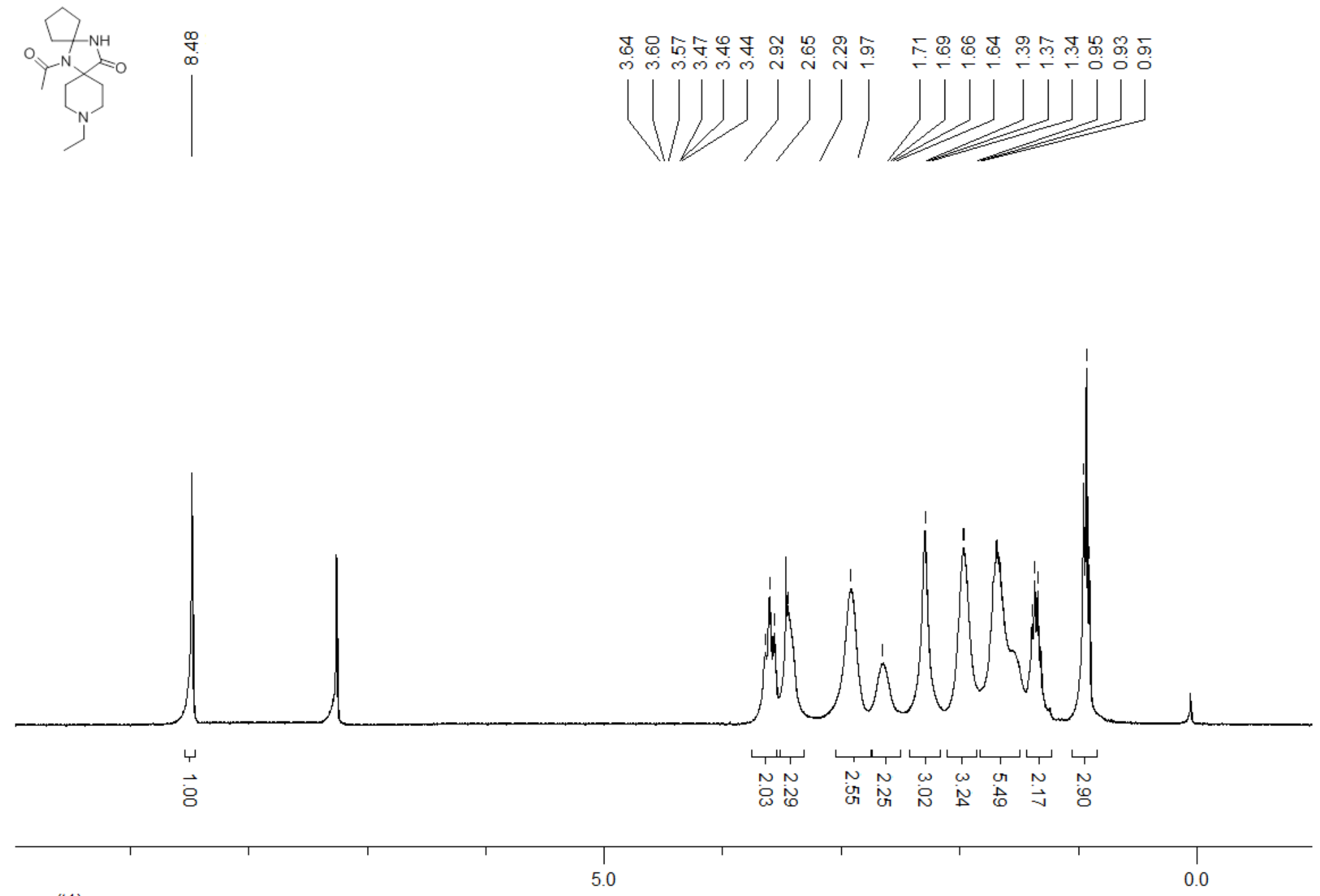

ppm (t1) 
HPLC-MS (ESI) 6-acetyl-10-ethyl-6,10,14-triazadispiro[4.1.5 $\left..2^{5}\right]$ tetradecan-13-one 8\{3.1\}

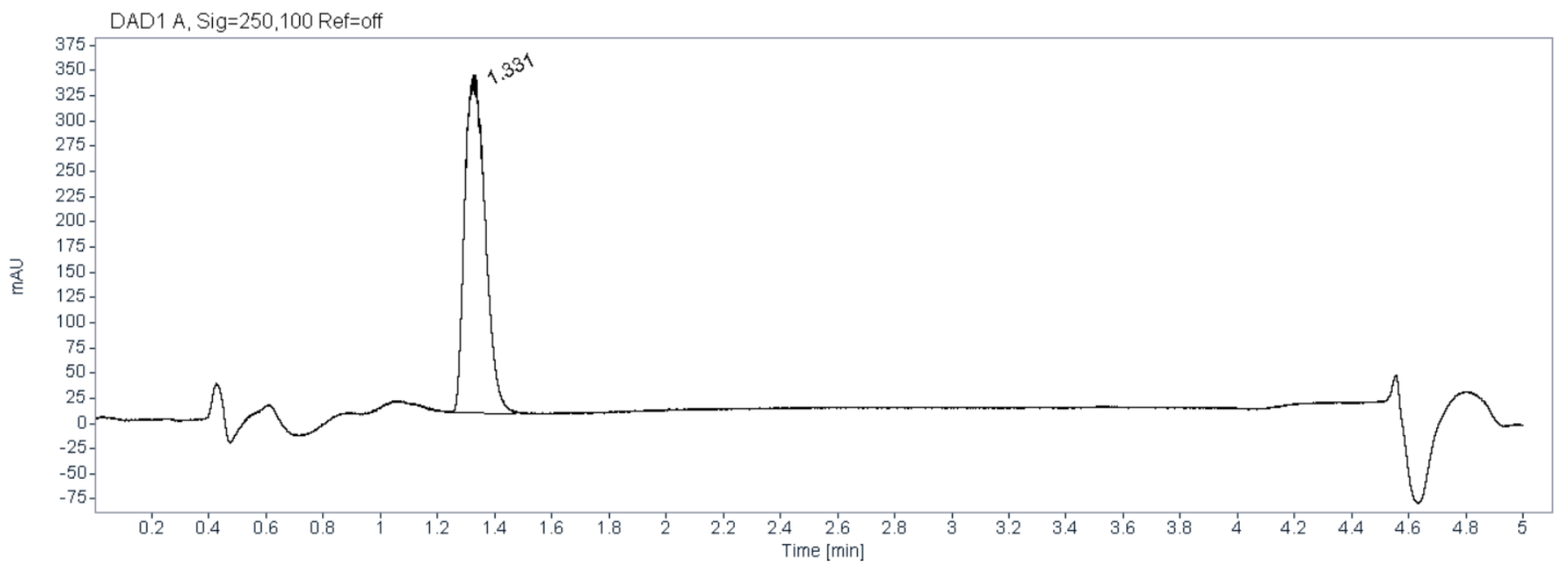

MSD1 SPC, MM-ES+APCI

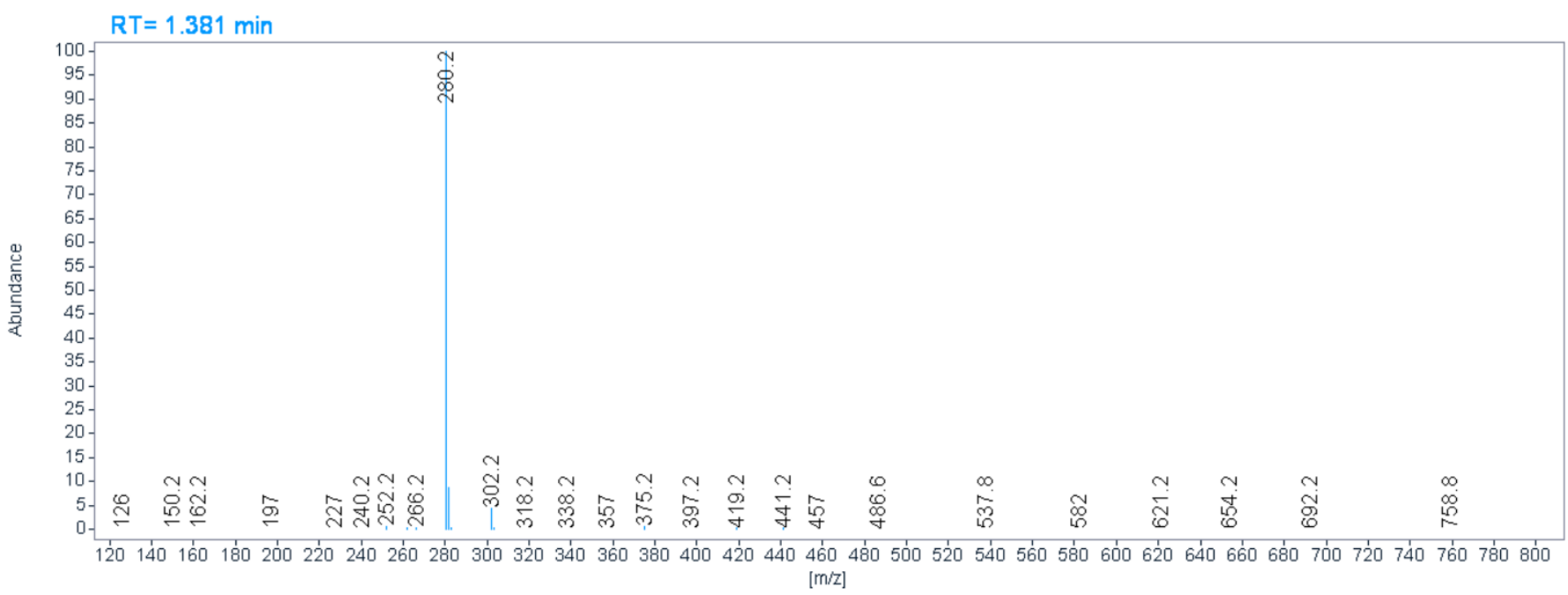


${ }^{1} \mathrm{H}$ NMR (300 MHz, CDCl 3 ) 6-acetyl-10-(3,3-dimethylbutyl)-6,10,14-triazadispiro[4.1.5 $\left.5^{7} 2^{5}\right]$ tetradecan-13-one 8\{3.2\}

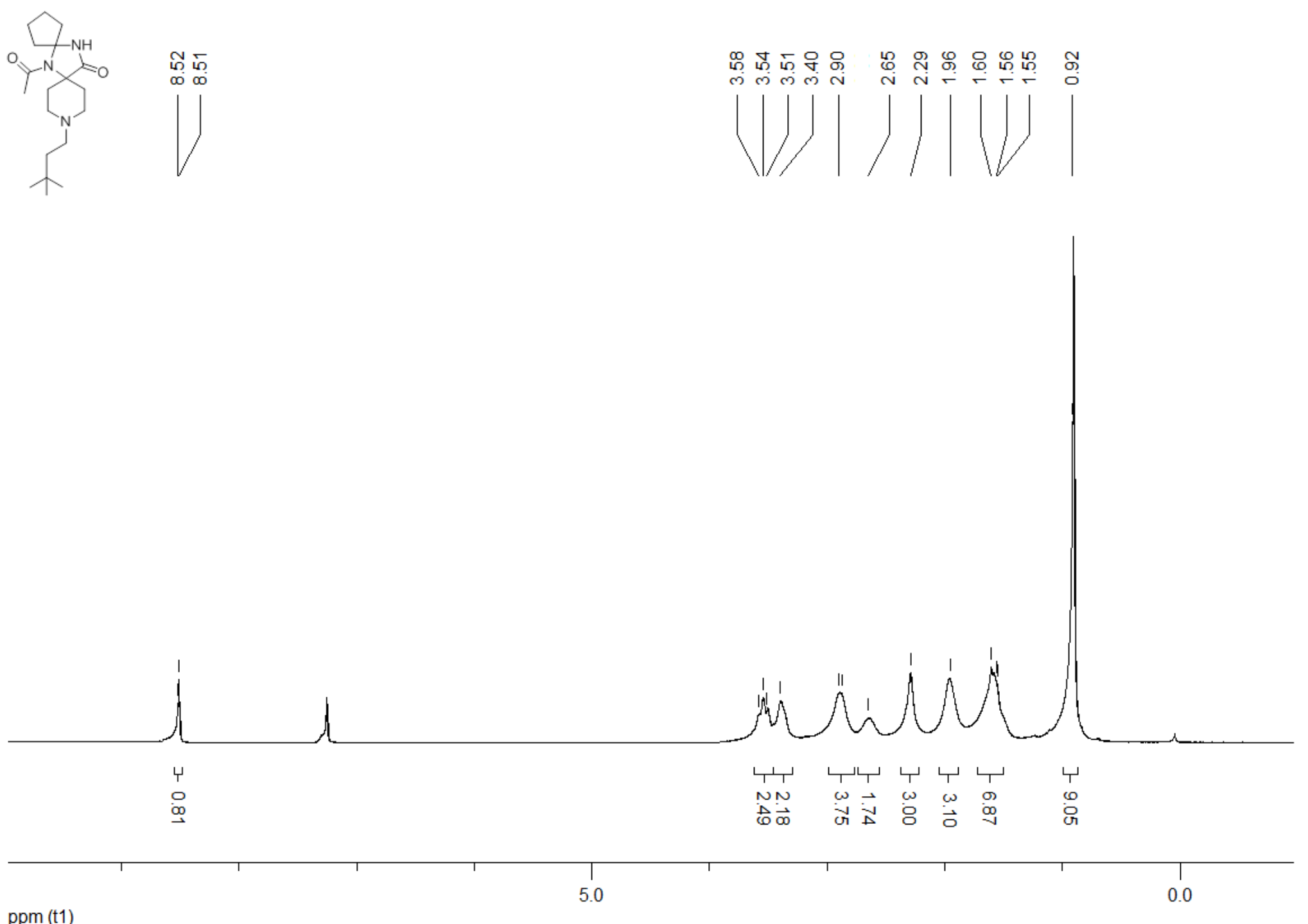


${ }^{1} \mathrm{H}$ NMR (300 MHz, $\mathrm{CDCl}_{3}$ ) 6-acetyl-10-[(4-ethoxy-3-methoxyphenyl)methyl]-6,10,14-triazadispiro[4.1.5 $\left..^{7} .^{5}\right]$ tetradecan-13-one 8 $\{3.3\}$

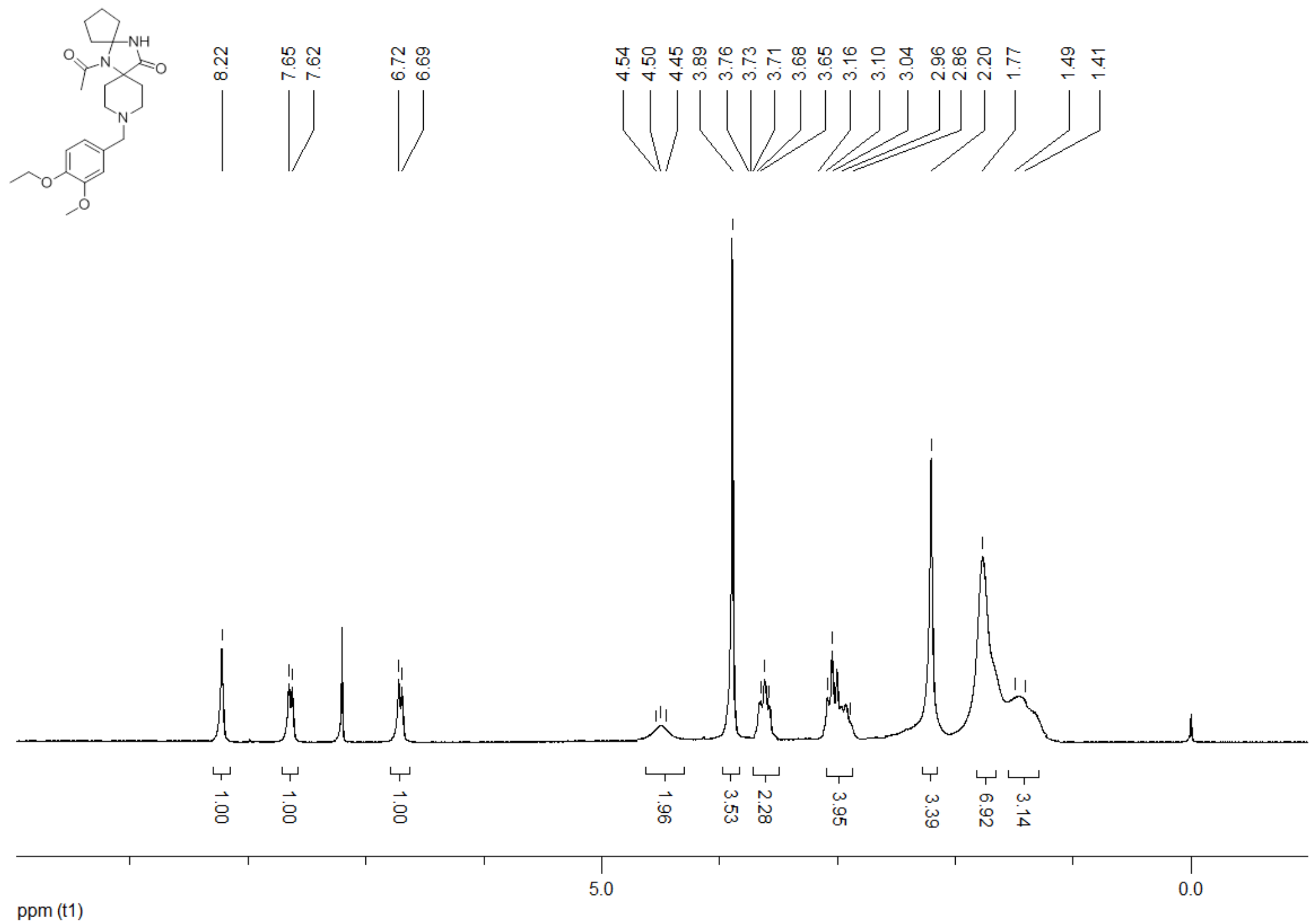


${ }^{1} \mathrm{H}$ NMR (300 MHz, $\mathrm{CDCl}_{3}$ ) 7-acetyl-N-benzyl-15-oxo-3,7,14-triazadispiro[5.1.5 ${ }^{8} .2^{6}$ ]pentadecane-3-carboxamide $10\{4.1\}$
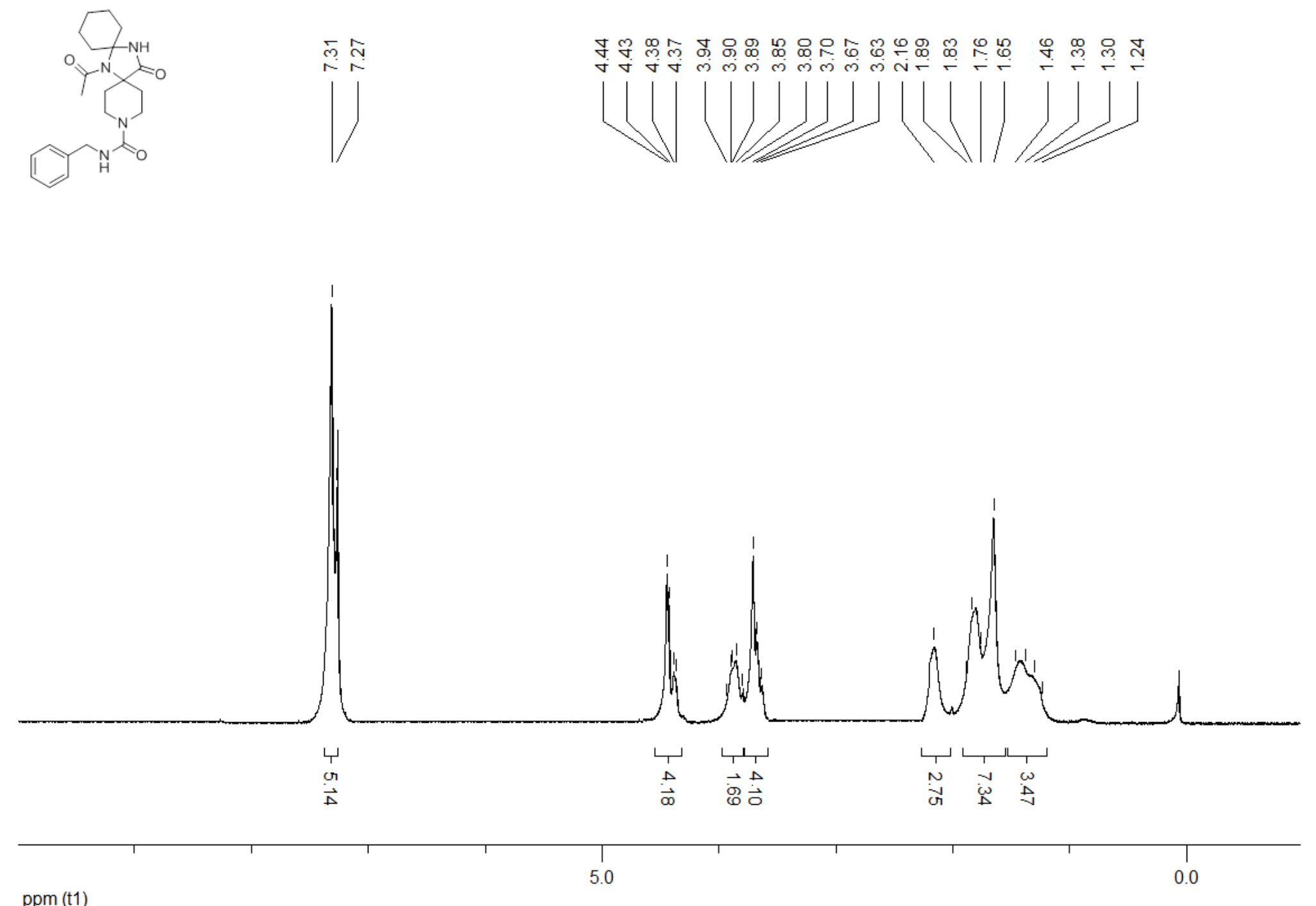
${ }^{1} \mathrm{H}$ NMR (300 MHz, $\mathrm{CDCl}_{3}$ ) 7-acetyl-15-oxo-N-[3-(trifluoromethyl)phenyl]-11-oxa-3,7,14-triazadispiro[5.1.5 $\left.{ }^{8} .^{6}\right]_{\text {pentadecane-3- }}$ carboxamide $10\{5.1\}$
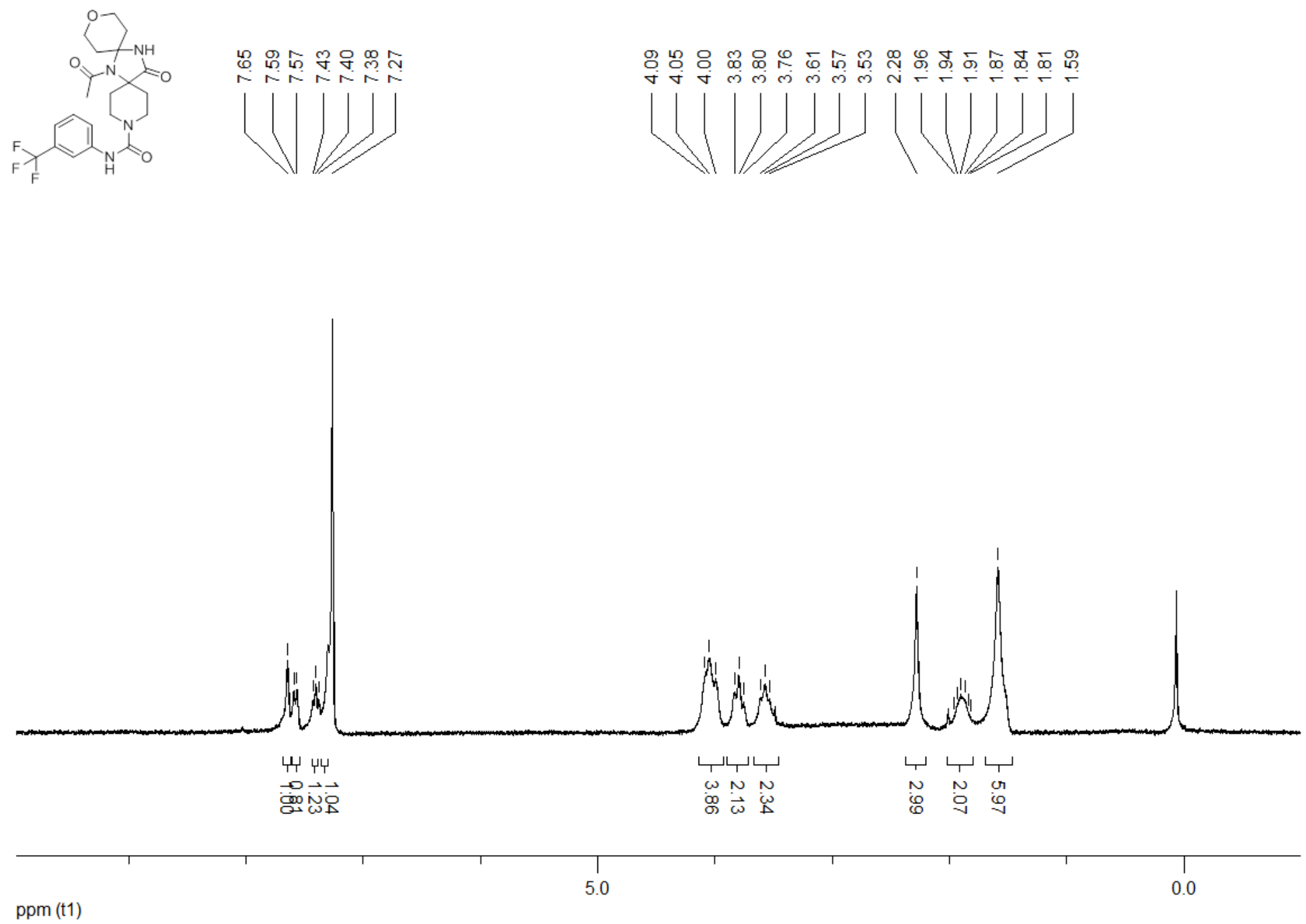
${ }^{1}$ H NMR (300 MHz, CDCl 3 ) 1-aminocyclopentanecarbonitrile $12\{3\}^{3}$

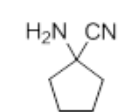

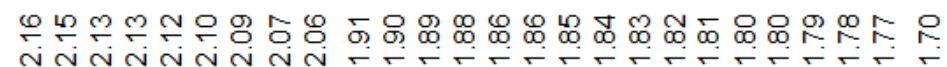
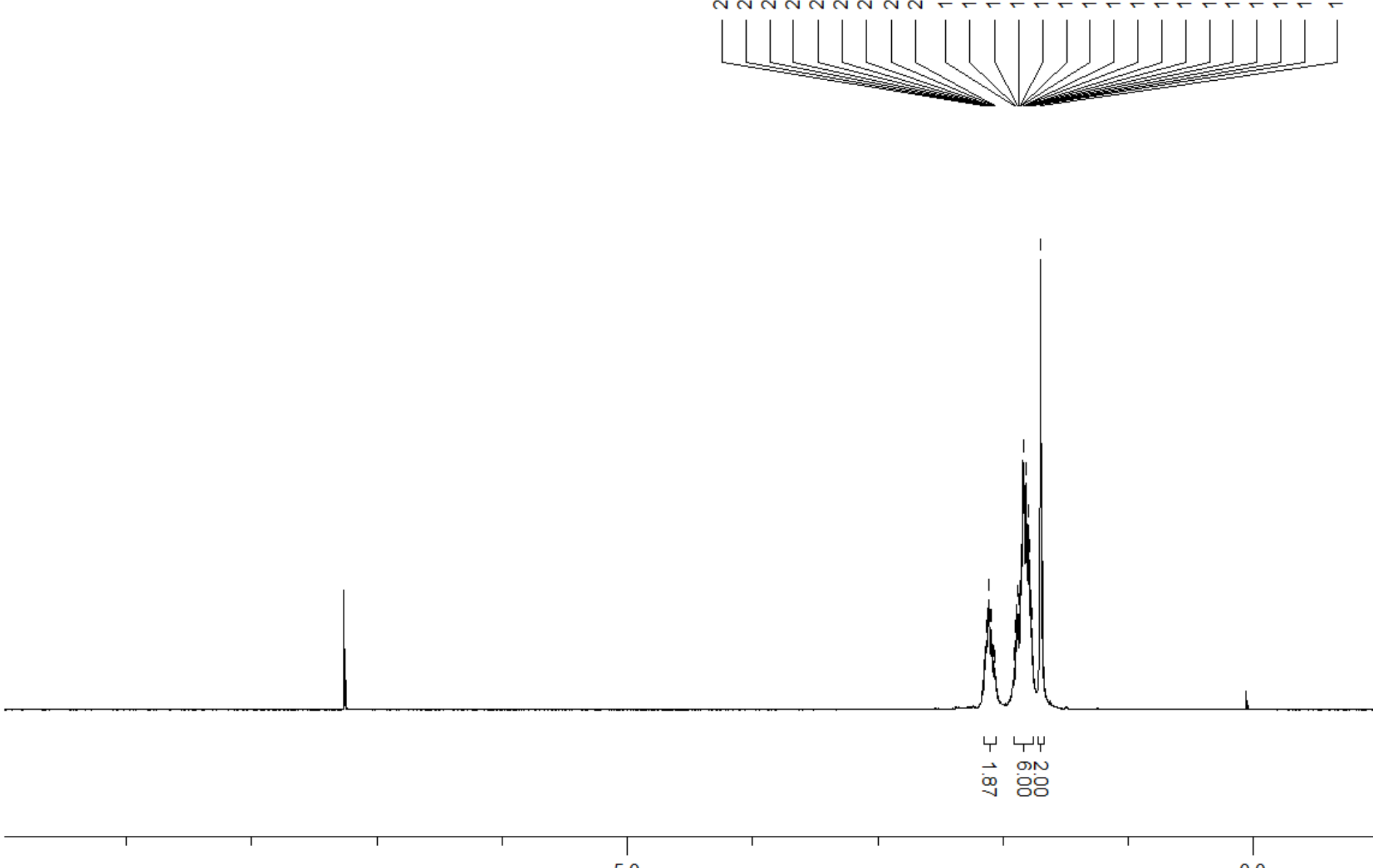

ppm (t1) 
${ }^{1}$ H NMR (300 MHz, CDCl 3 ) 4-aminooxane-4-carbonitrile $12\{5\}^{4}$

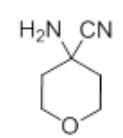

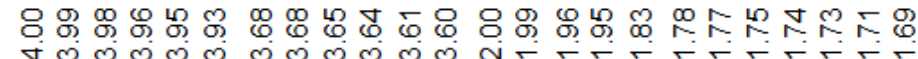

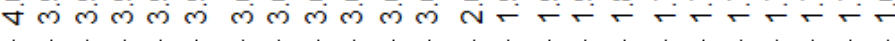
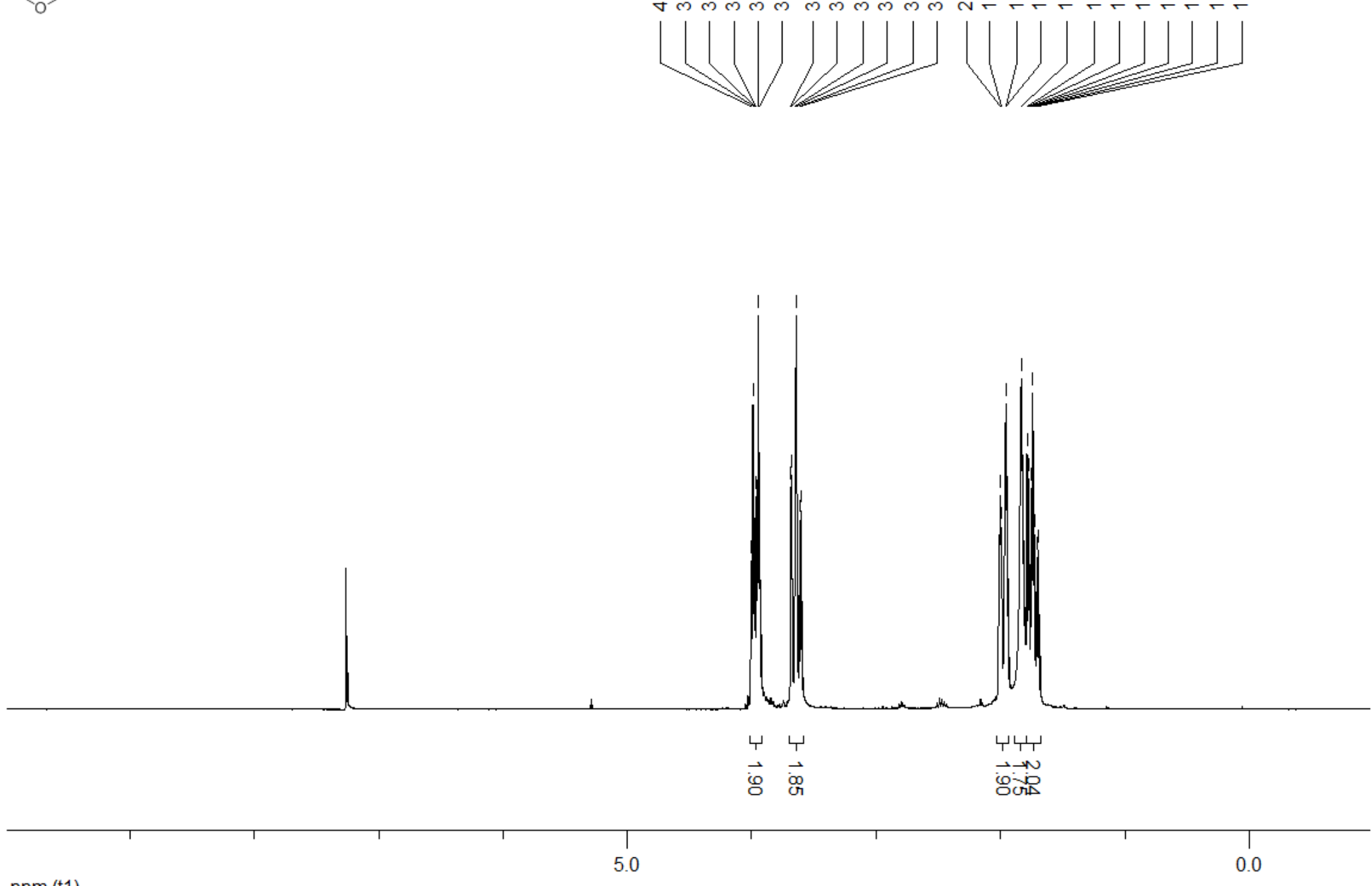

ppm (t1) 
${ }^{1}$ H NMR (300 MHz, CDCI3) 4-amino-1-methylpiperidine-4-carbonitrile $12\{6\}^{5}$
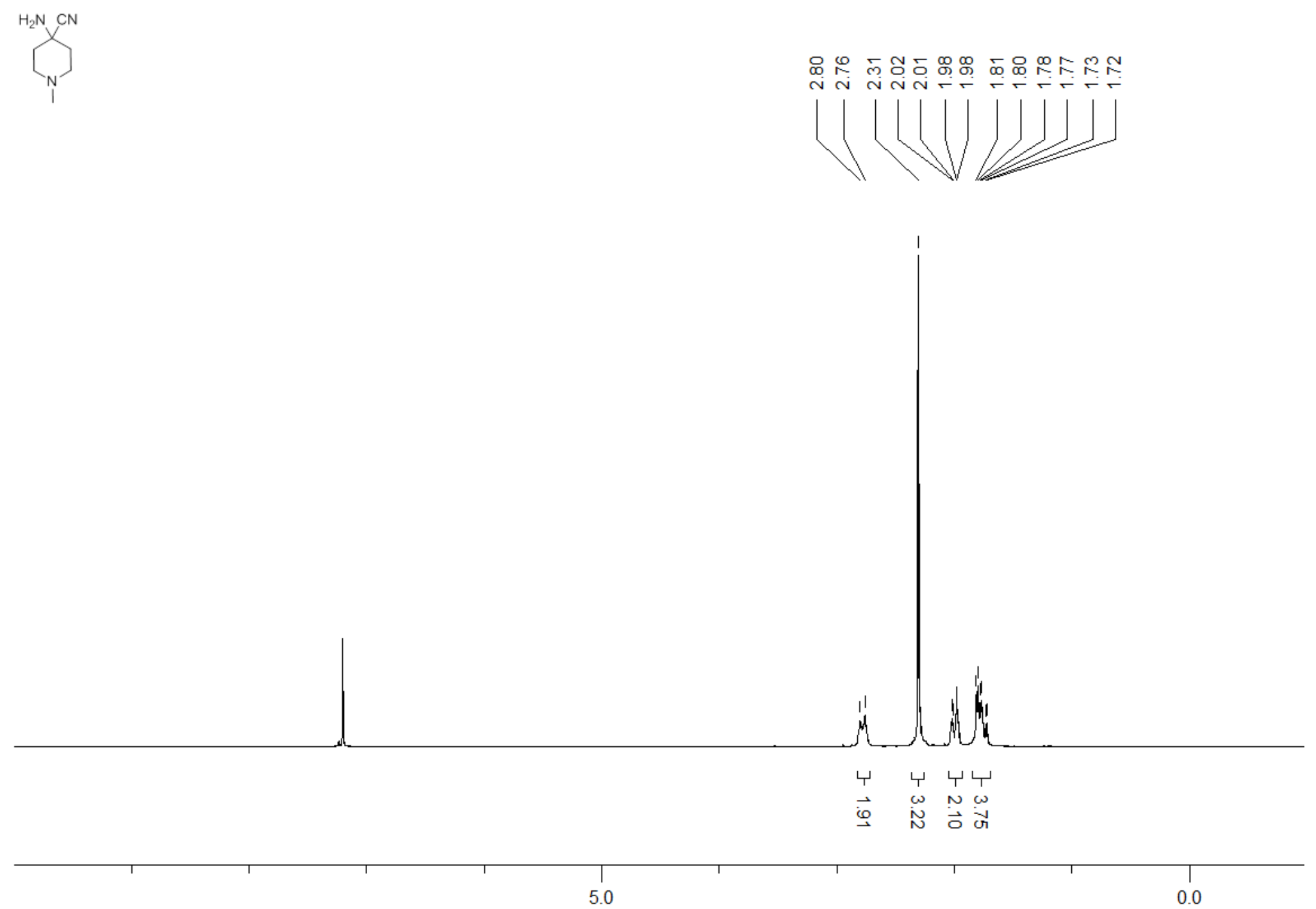

ppm (t1) 
${ }^{1}$ H NMR (300 MHz, CDCl3) 1-aminocycloheptanecarbonitrile $12\{7\}^{6}$

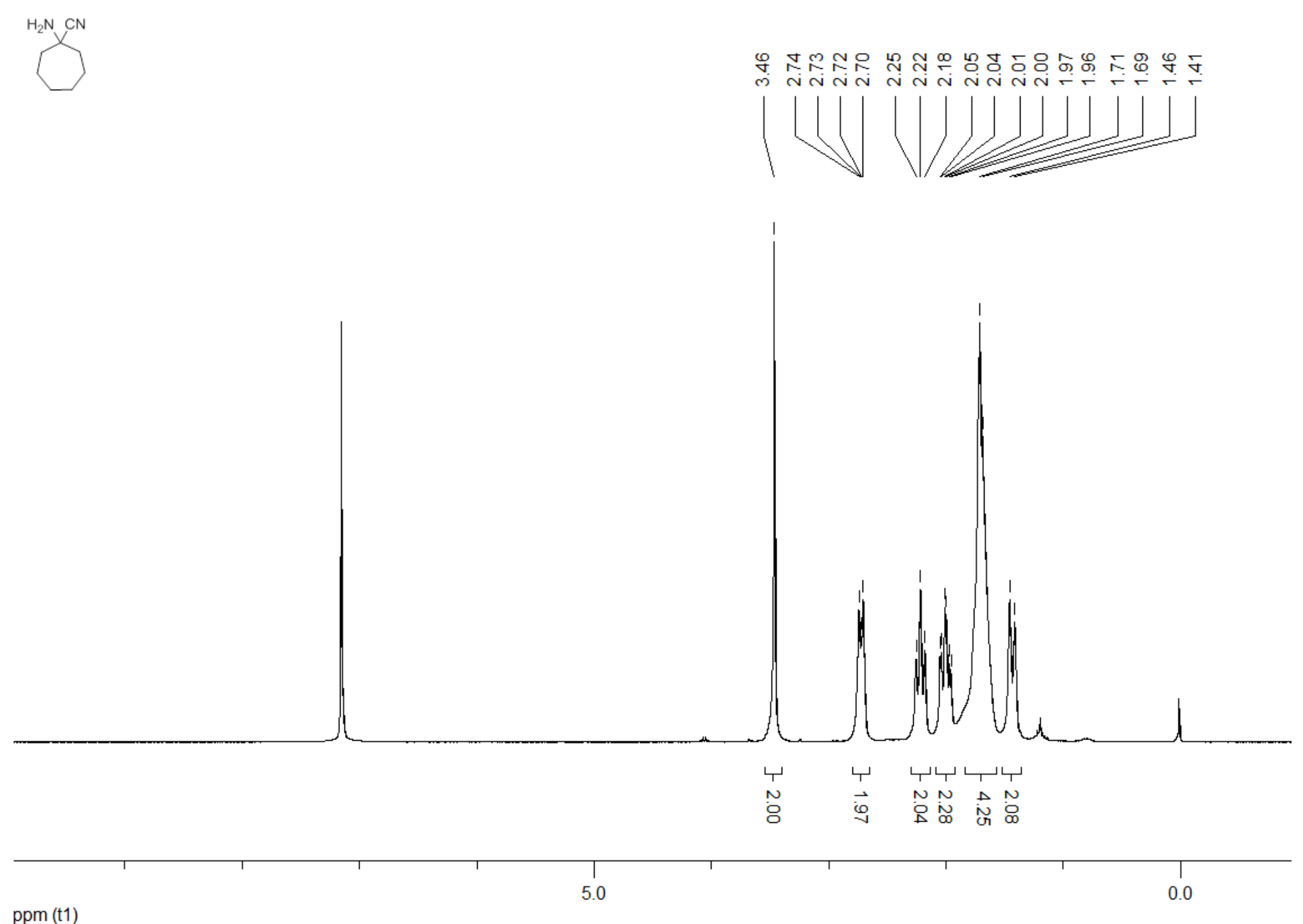


${ }^{1} \mathrm{H}$ NMR (300 MHz, D 20 ) 1-aminocyclopentane-1-carboxylic acid $13\{3\}^{7}$

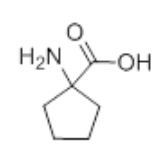

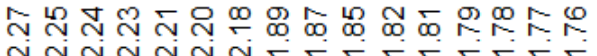
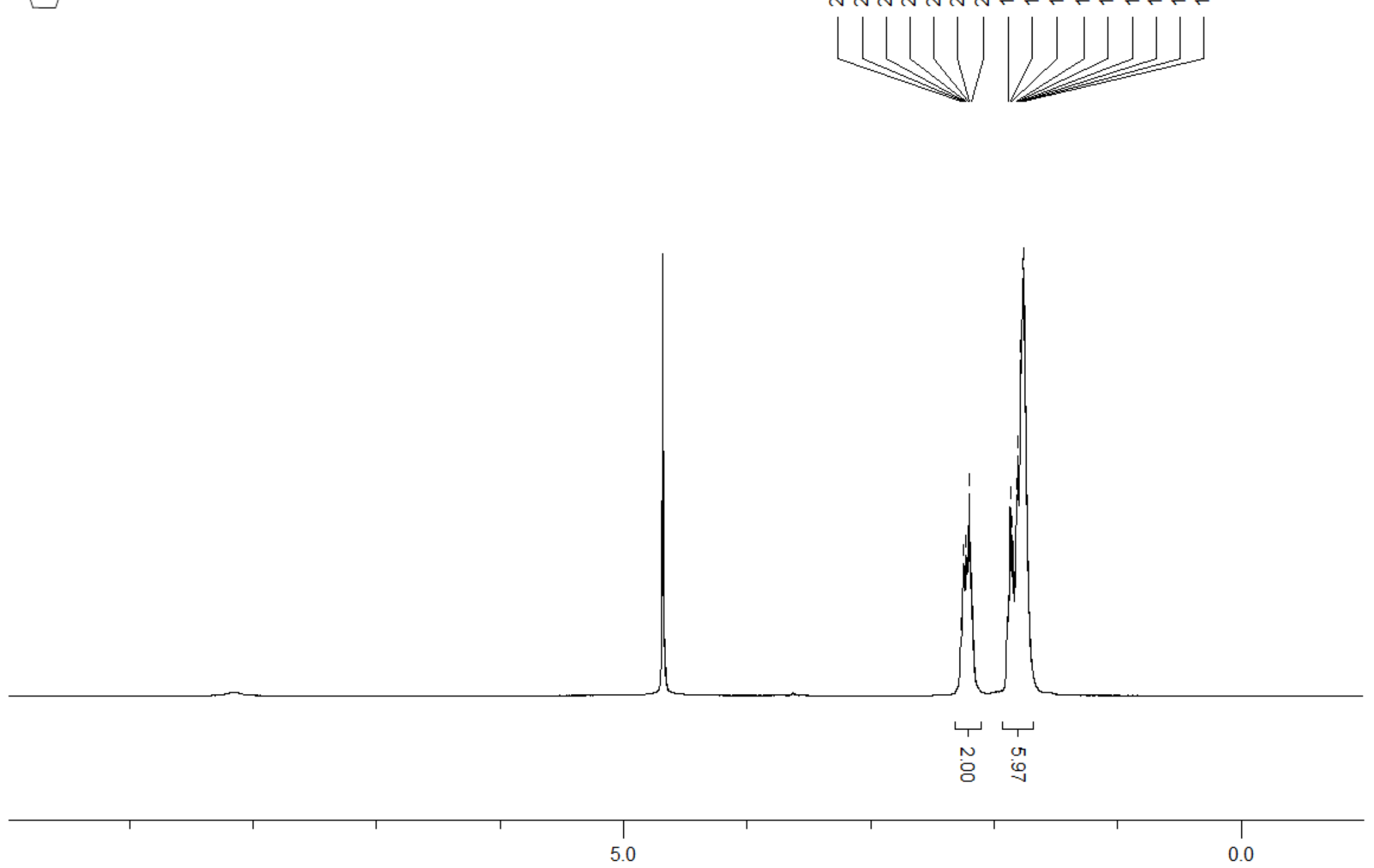

ppm (t1) 
${ }^{1} \mathrm{H}$ NMR (300 MHz, D 20 ) 4-aminooxane-4-carboxylic acid $13\{5\}^{8}$

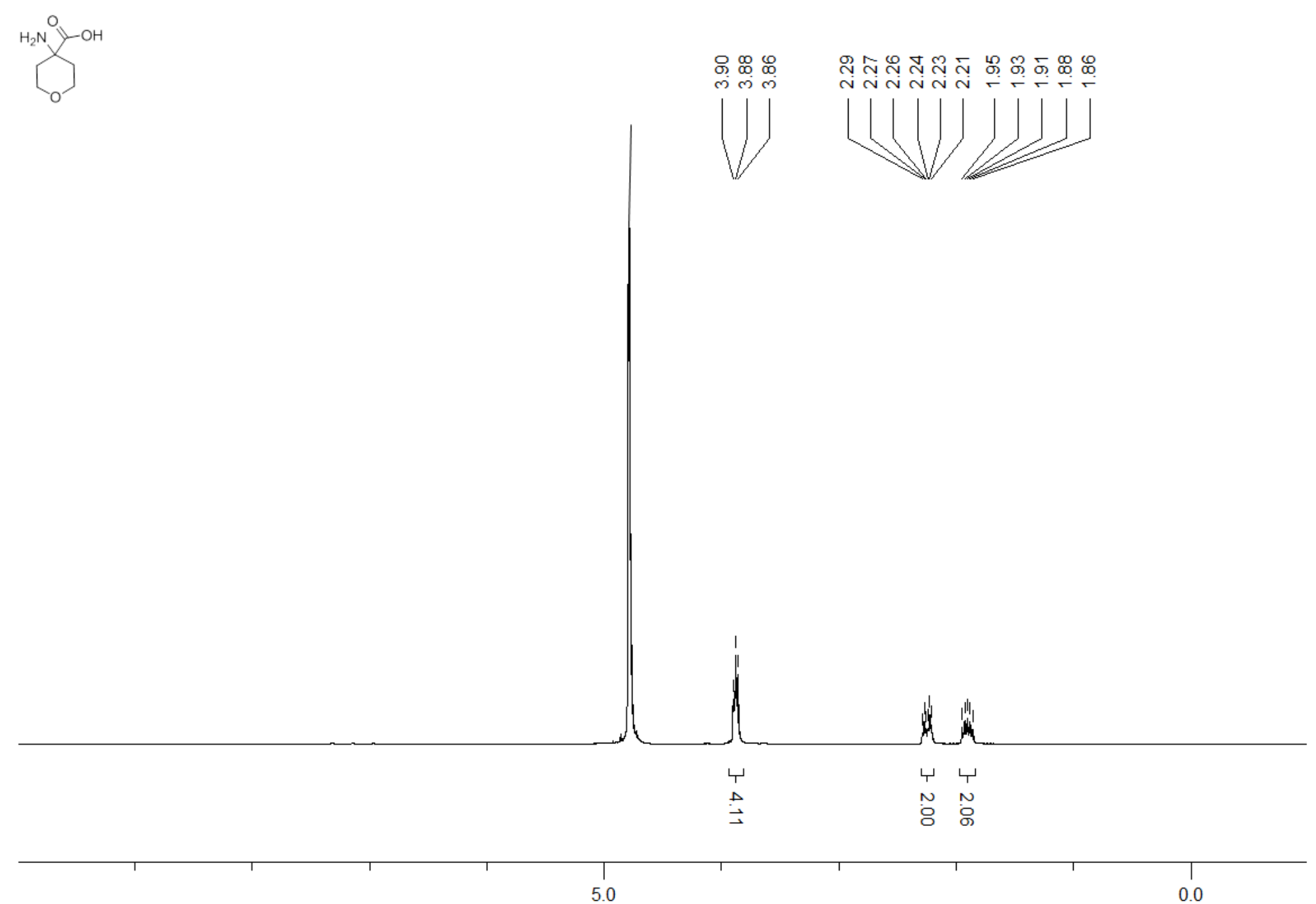

ppm (t1) 
${ }^{1}$ H NMR (300 MHz, D 2 O) 1-aminocycloheptane-1-carboxylic acid $13\{7\}^{7}$

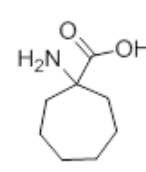

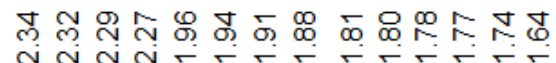
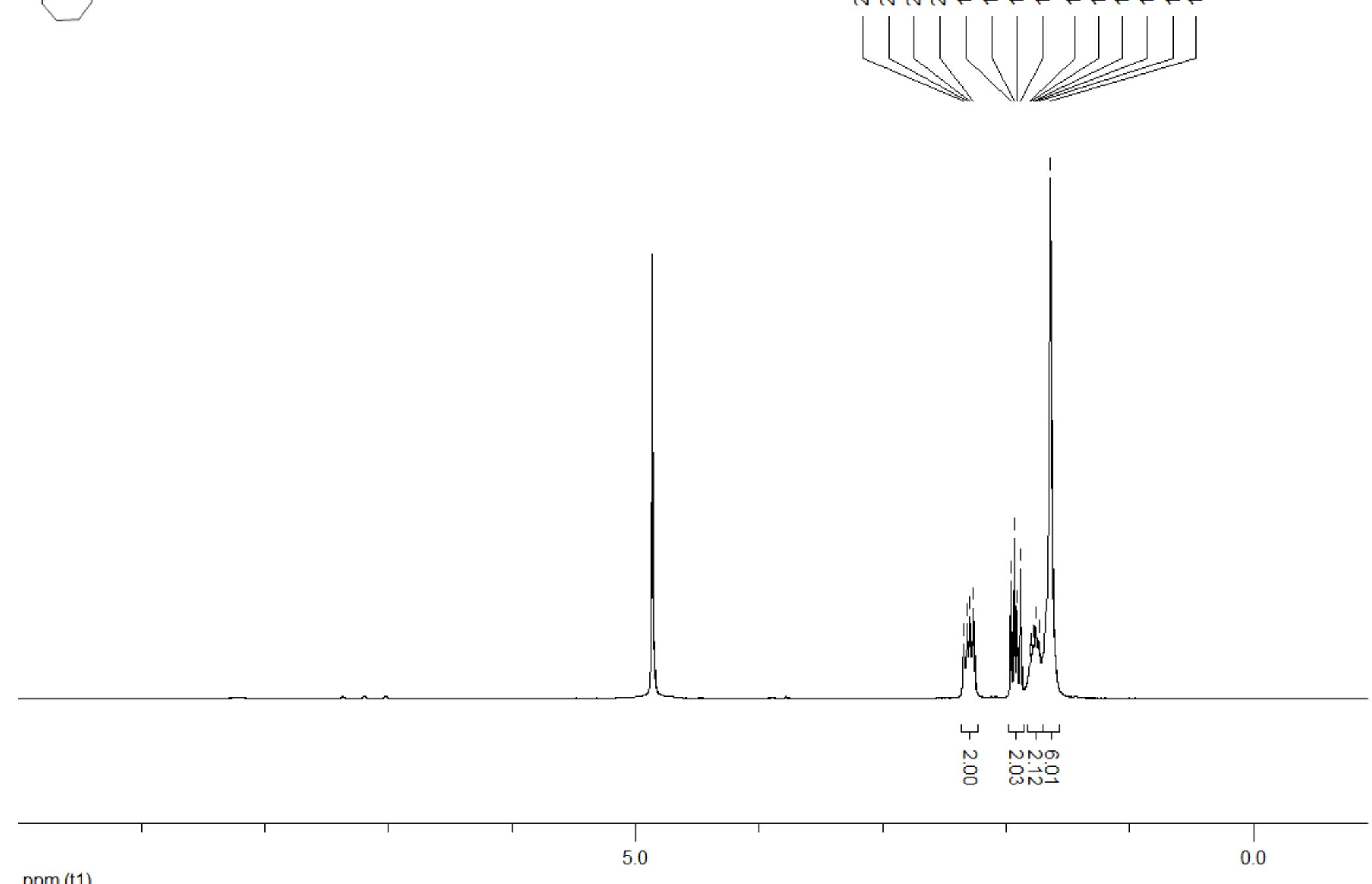
${ }^{1} \mathrm{H}$ NMR (300 MHz, D 20 ) methyl 1-aminocyclopentane-1-carboxylate $14\{3\}^{7}$

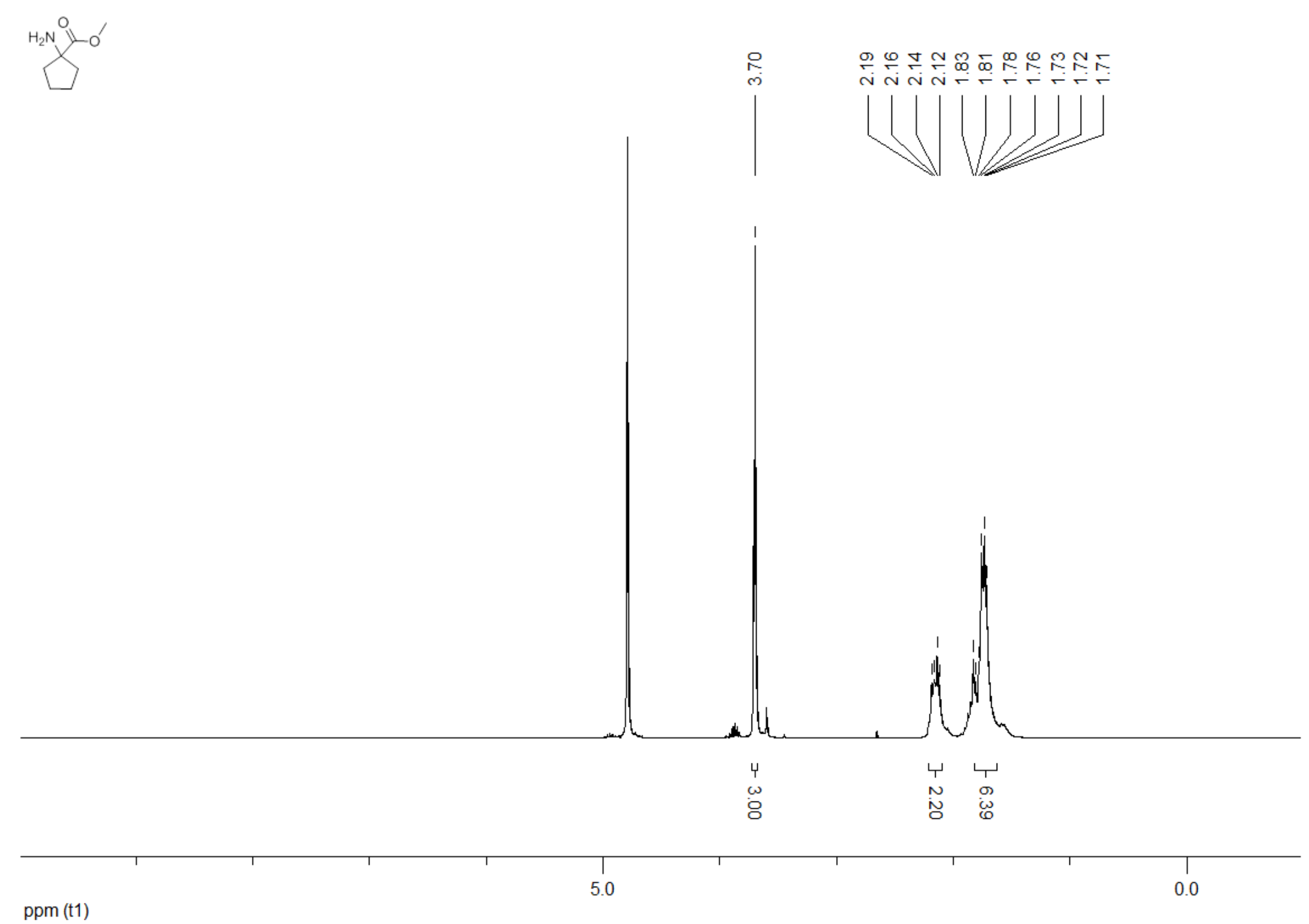


HPLC-MS (ESI) methyl 1-aminocyclopentane-1-carboxylate $14\{3\}^{7}$

\section{Retention Time : 1.228 minutes}

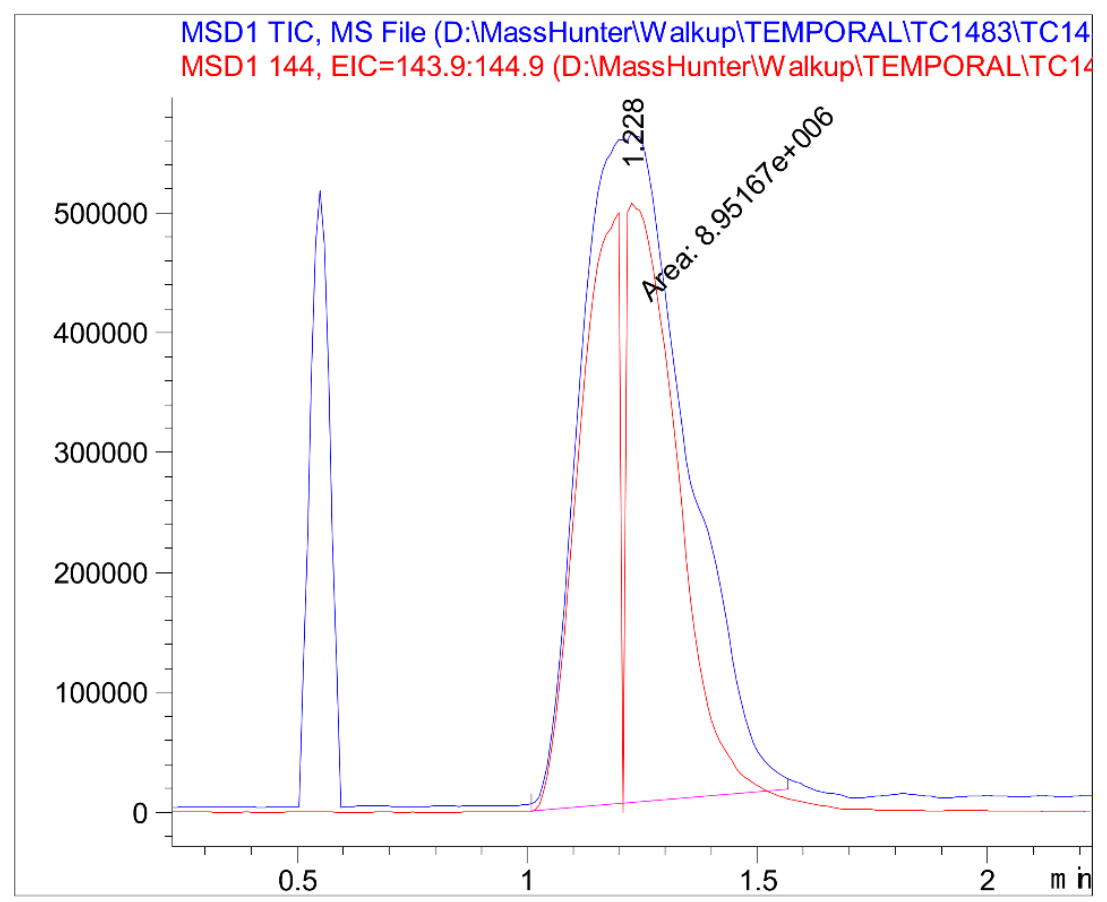

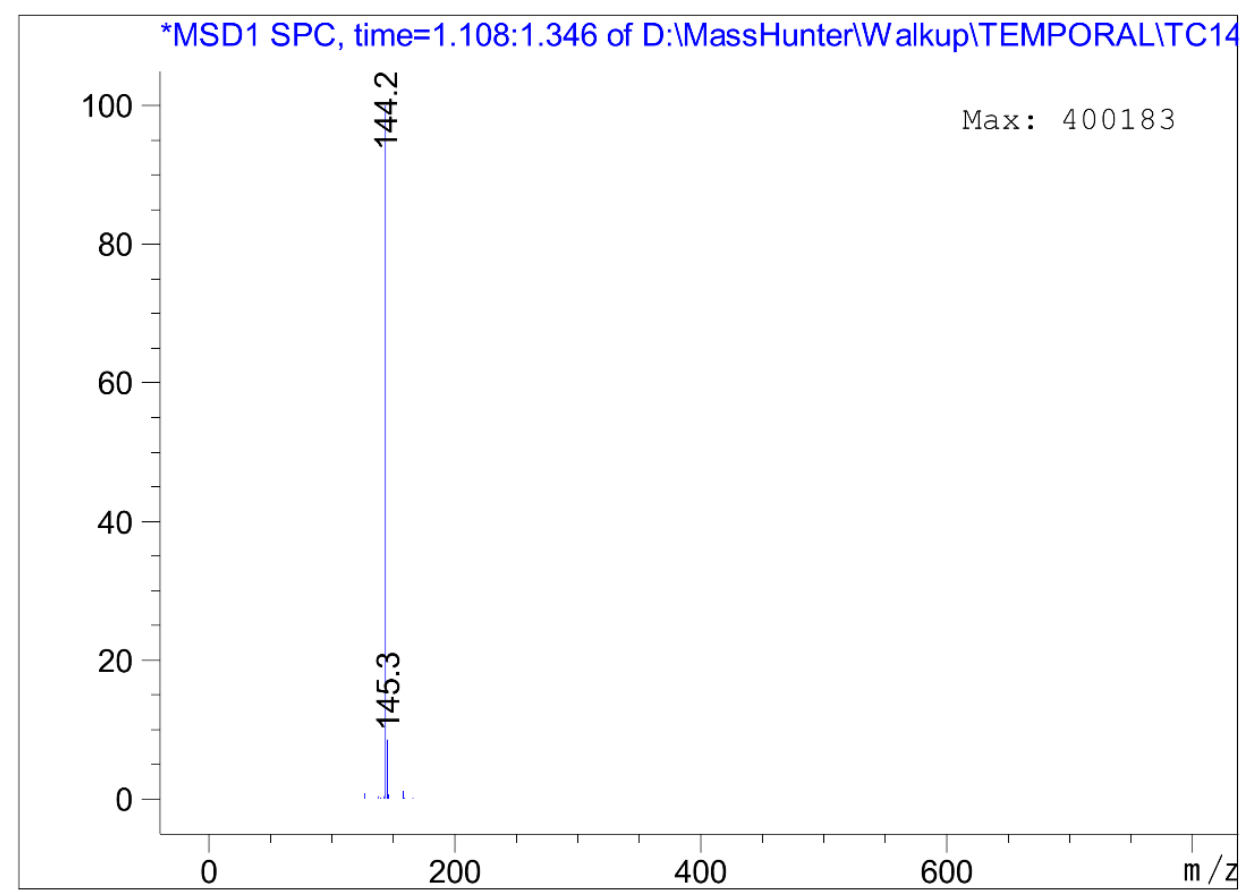


${ }^{1}$ H NMR (300 MHz, CDCl 3 ) methyl 1-aminocyclohexane-1-carboxylate $14\{4\}^{9}$
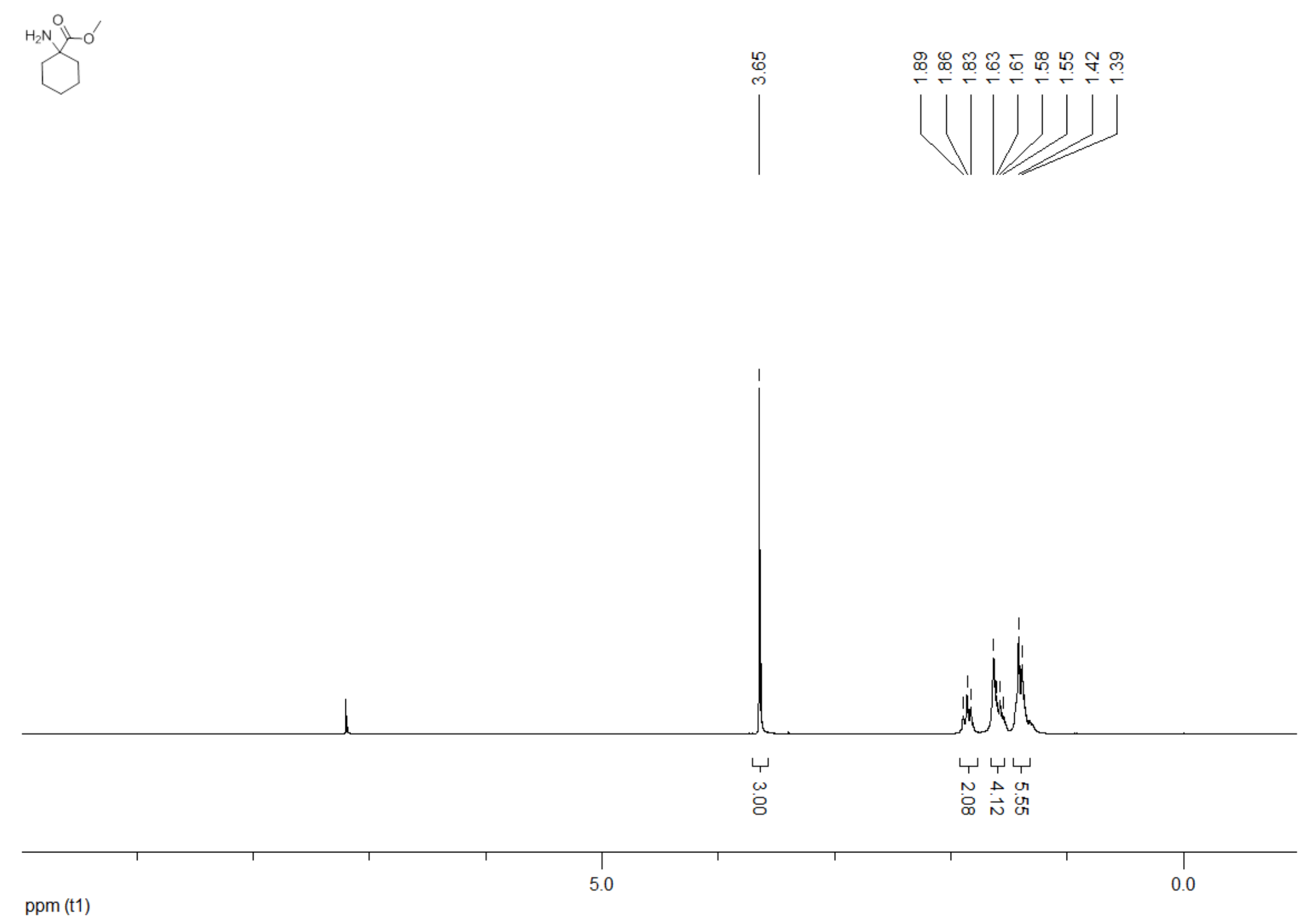
HPLC-MS (ESI) methyl 1-aminocyclohexane-1-carboxylate $14\{4\}^{9}$

Retention Time : 1.366 minutes

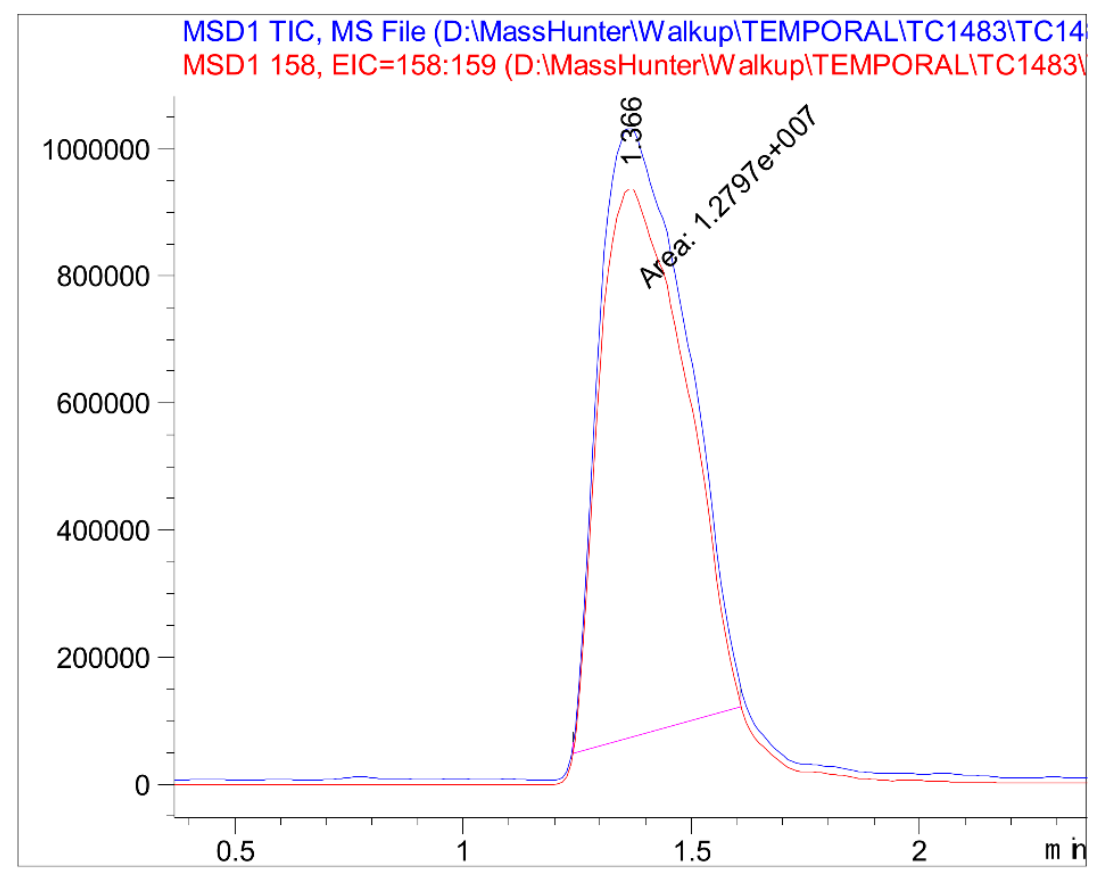

${ }^{*}$ MSD1 SPC, time=1.291:1.520 of D:IMassHunterlWalkupITEMPORALITC14

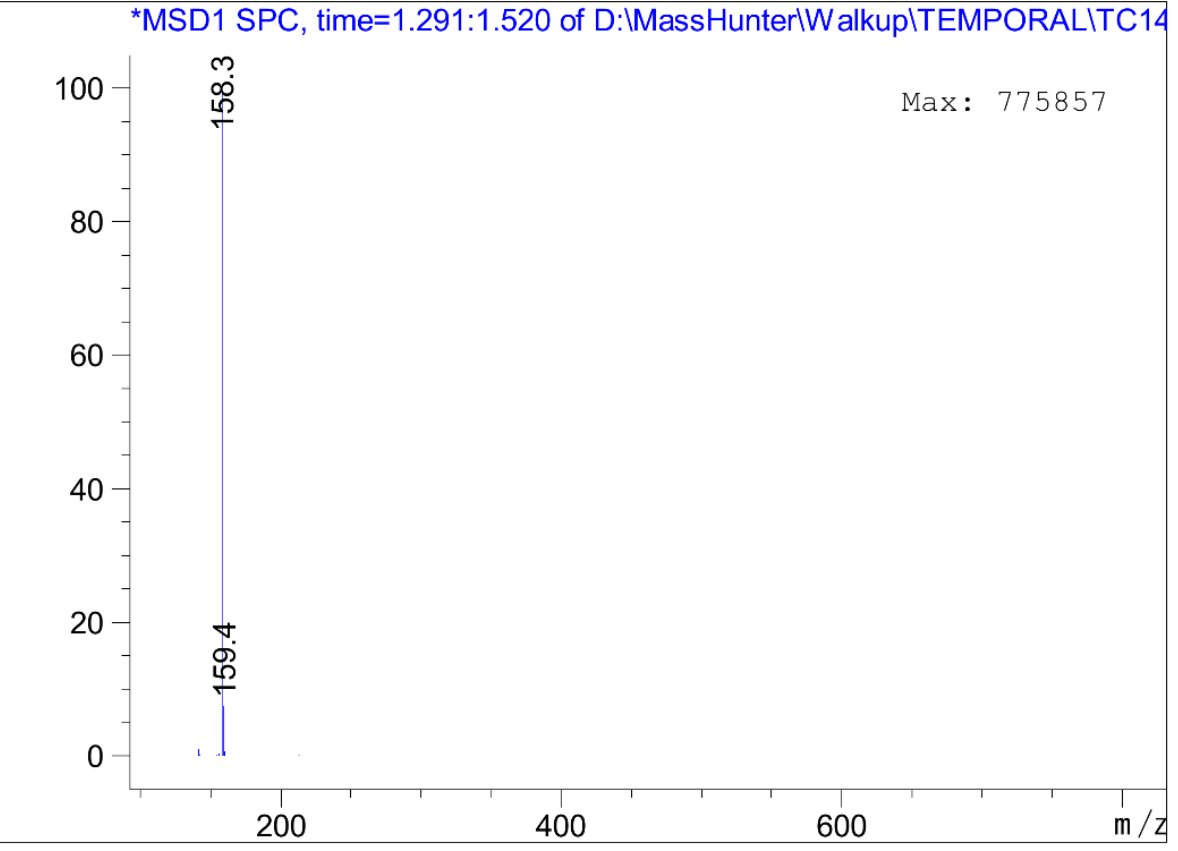


${ }^{1} \mathrm{H}$ NMR (300 MHz, D 20 ) methyl-4-aminooxane-4-carboxylate $14\{5\}^{8}$

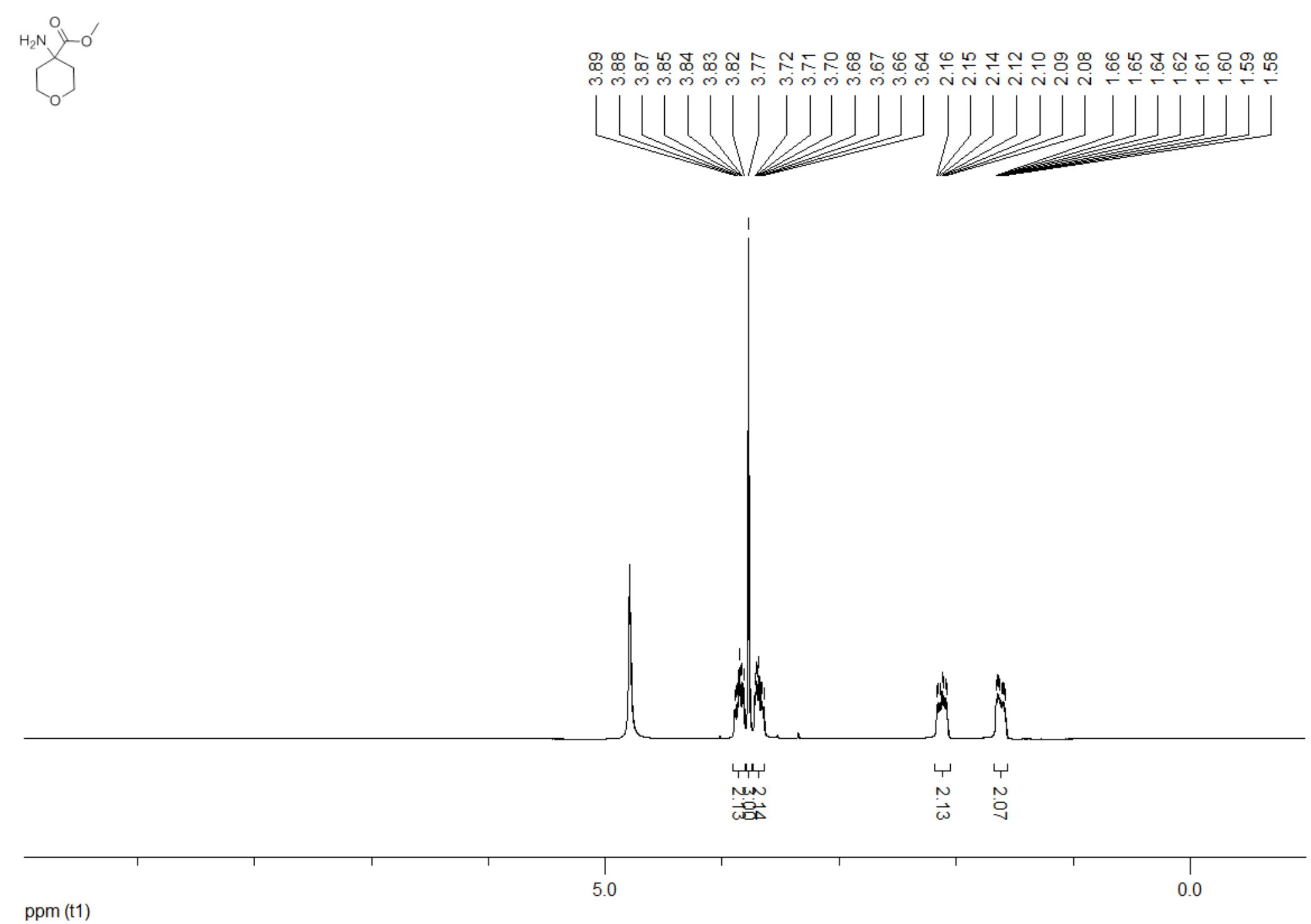


HPLC-MS (ESI) methyl-4-aminooxane-4-carboxylate $14\{5\}^{8}$

\section{Retention Time : 1.366 minutes}
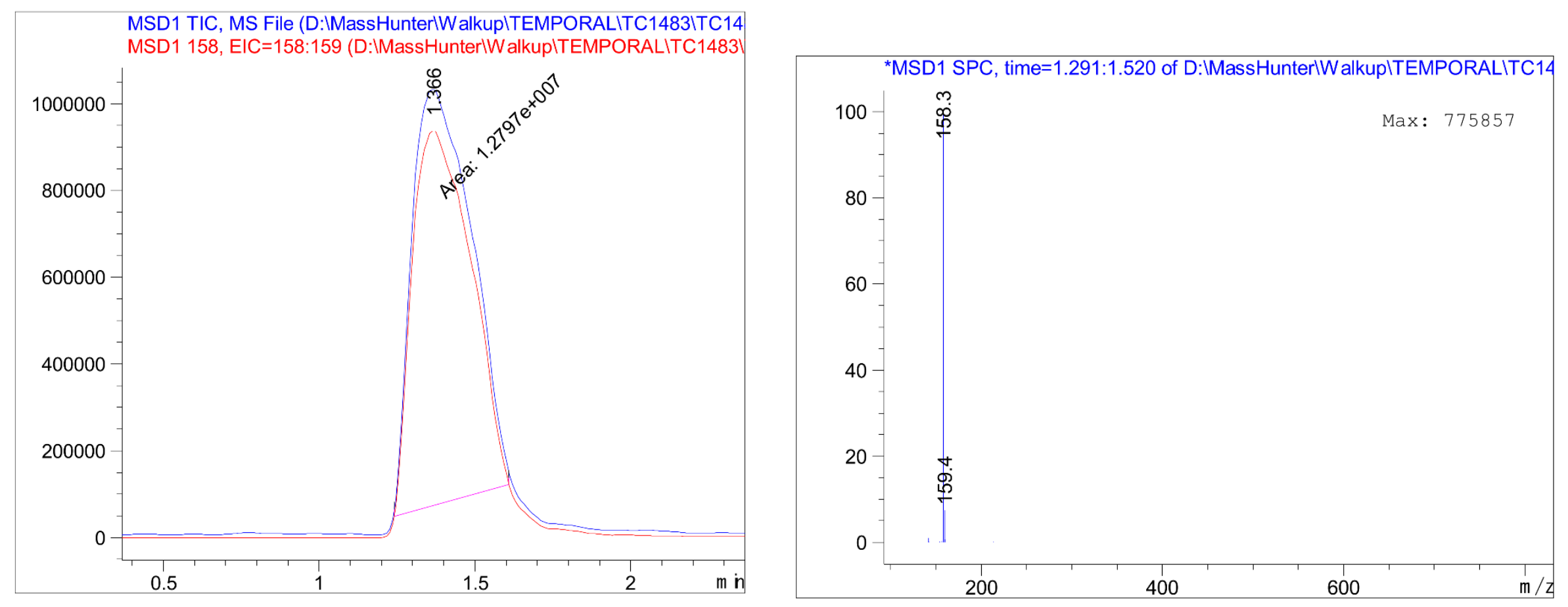
${ }^{1}$ H NMR (300 MHz, $\left.\mathrm{CDCl}_{3}\right)$ (1-aminocyclopentyl)methanol $15\{3\}^{10}$

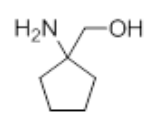

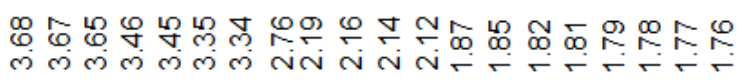
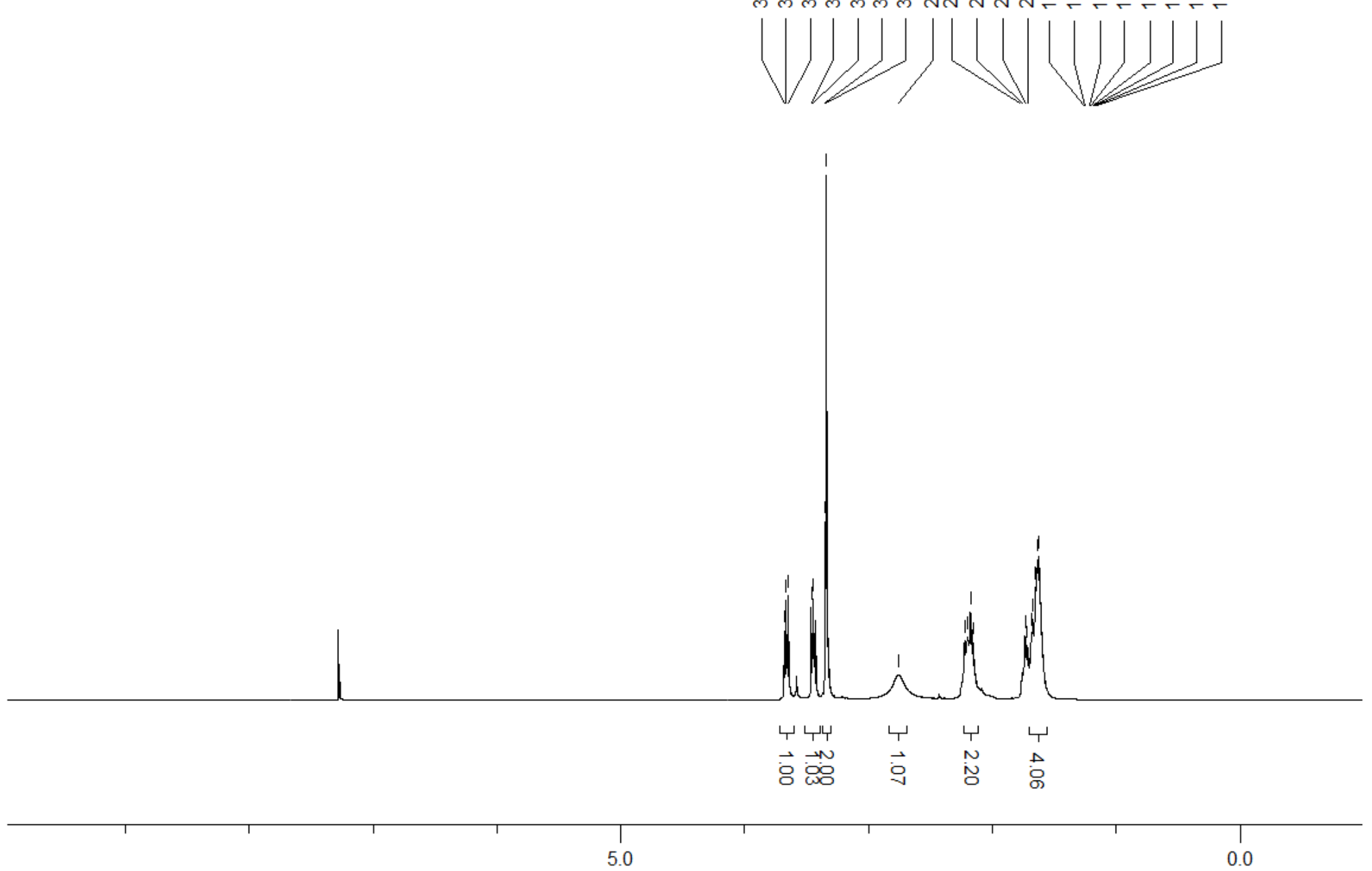

ppm (t1) 
${ }^{1}$ H NMR (300 MHz, CDCl $)$ (1-aminocyclohexyl)methanol $15\{4\}^{11}$

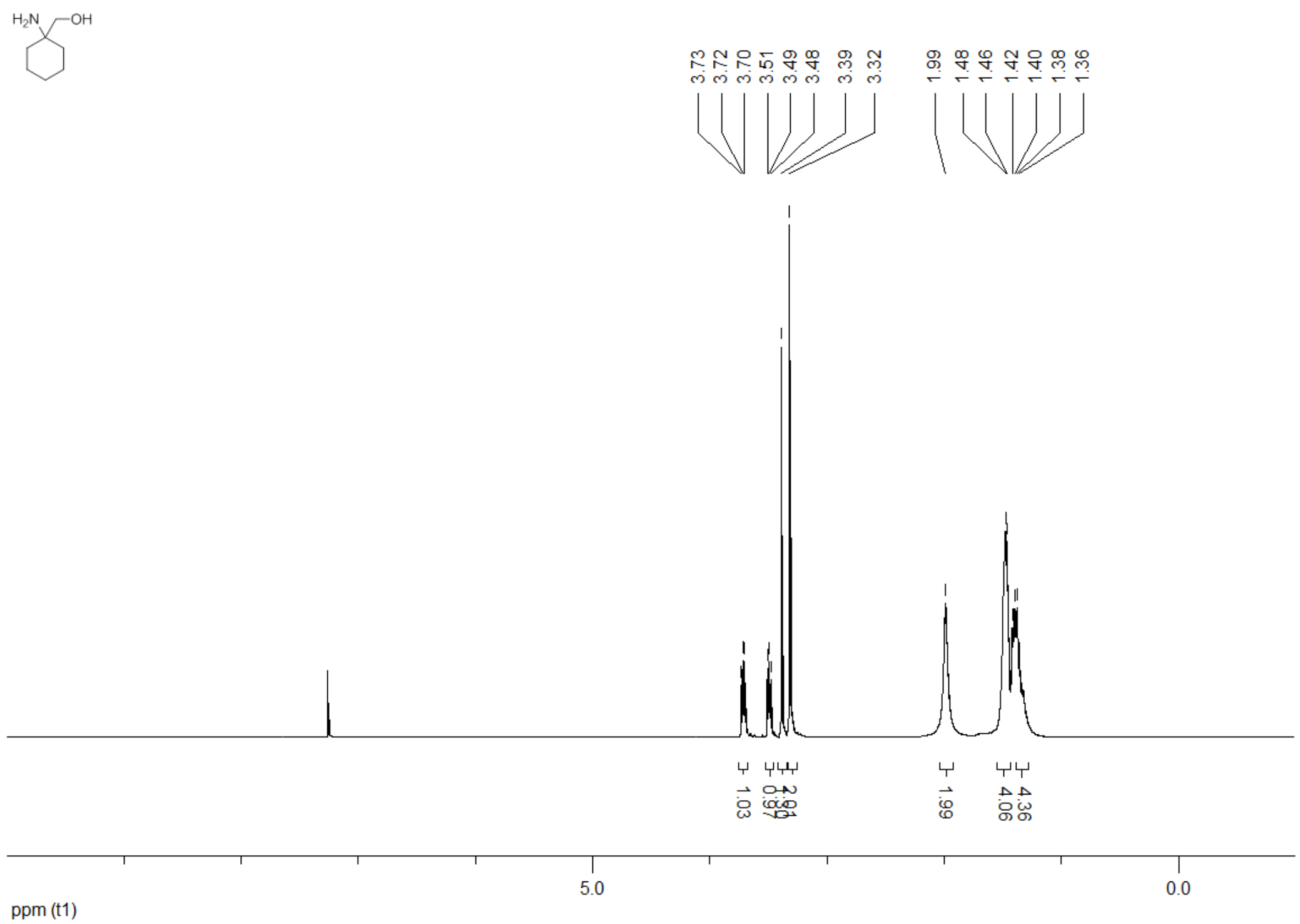


${ }^{1}$ H NMR (300 MHz, CDCl $)$ (4-aminooxan-4-yl)methanol $15\{5\}^{10}$

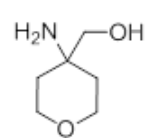

ஜ
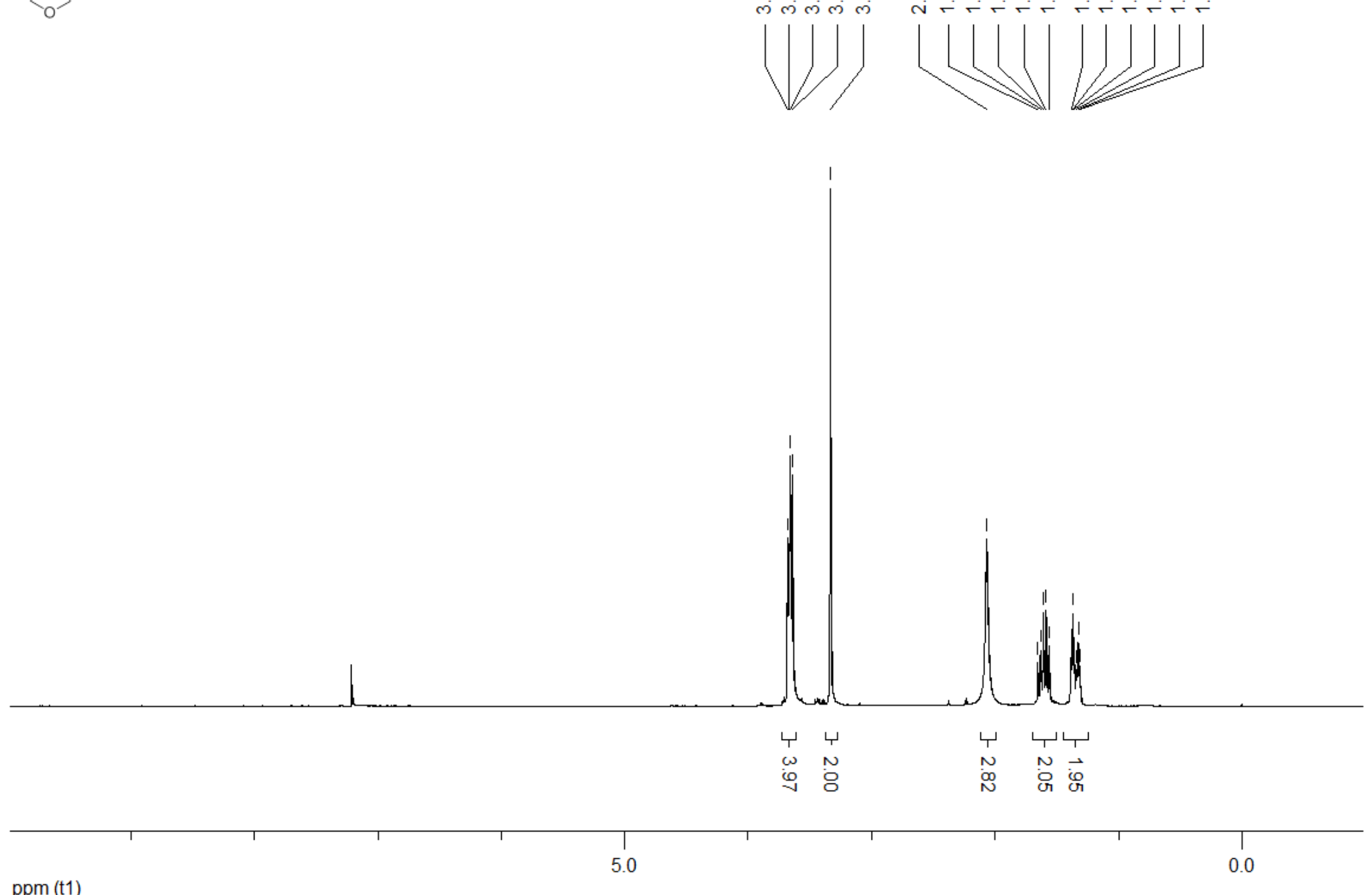
HPLC-MS (ESI) (4-aminooxan-4-yl)methanol 15\{5 $\}^{10}$

Retention Time : 0.488 minutes
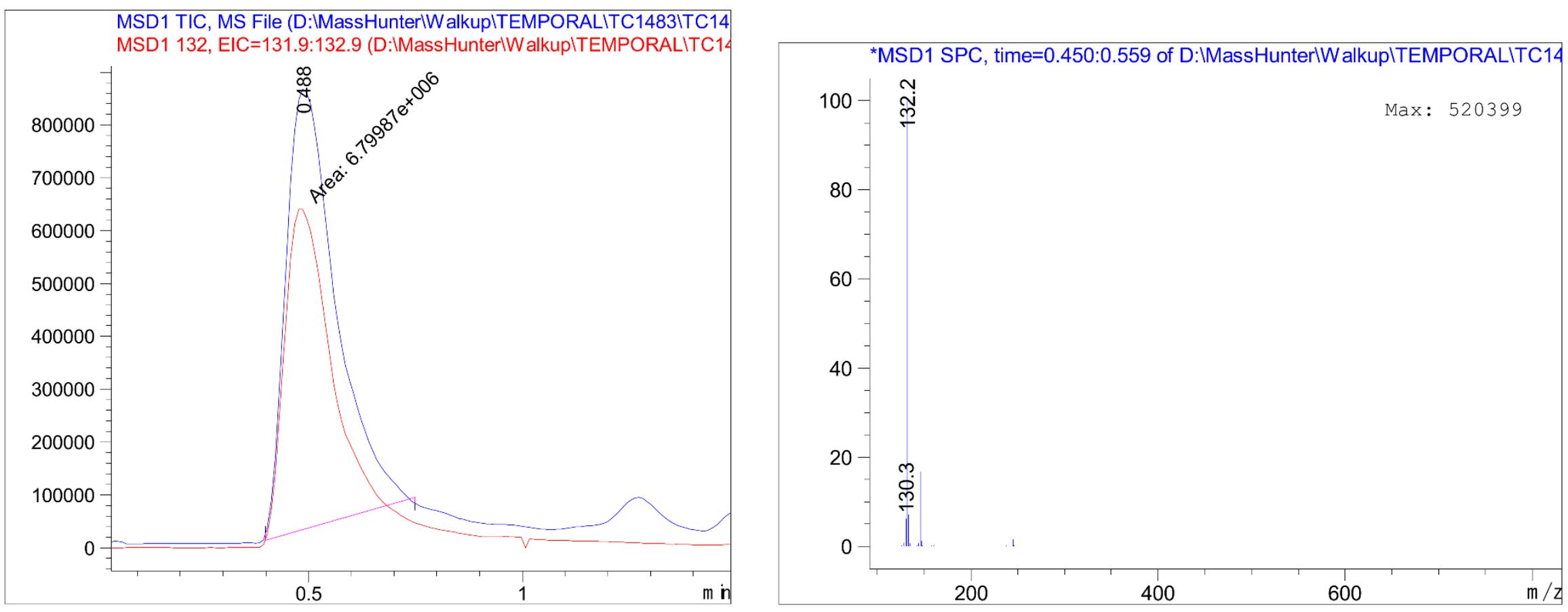
${ }^{1}$ H NMR (300 MHz, CDCl 3 ) 8-benzyl-3,3-dimethyl-1-oxa-4,8-diazaspiro[4.5]decane 16\{1\}
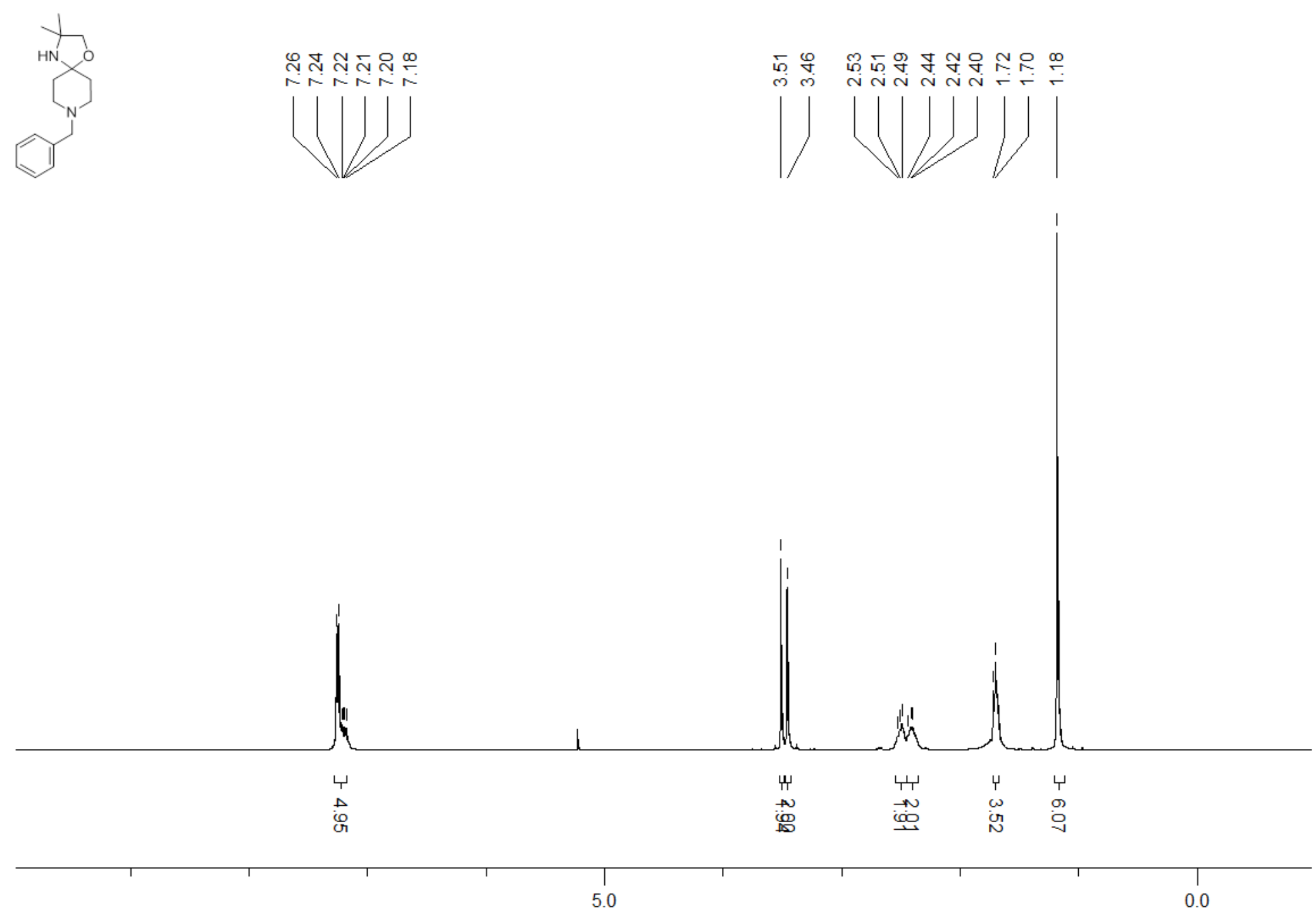

ppm (t1) 
HPLC-MS (ESI) 8-benzyl-3,3-dimethyl-1-oxa-4,8-diazaspiro[4.5]decane 16\{1\}

\section{Retention Time : 1.394 minutes}

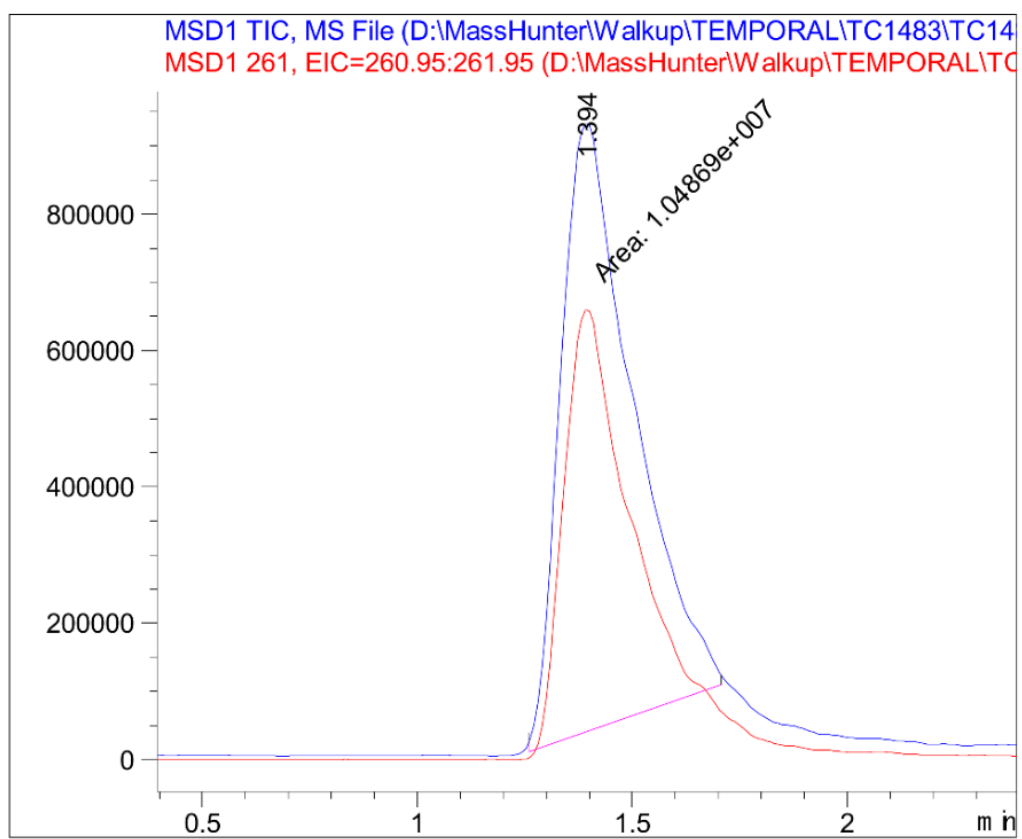

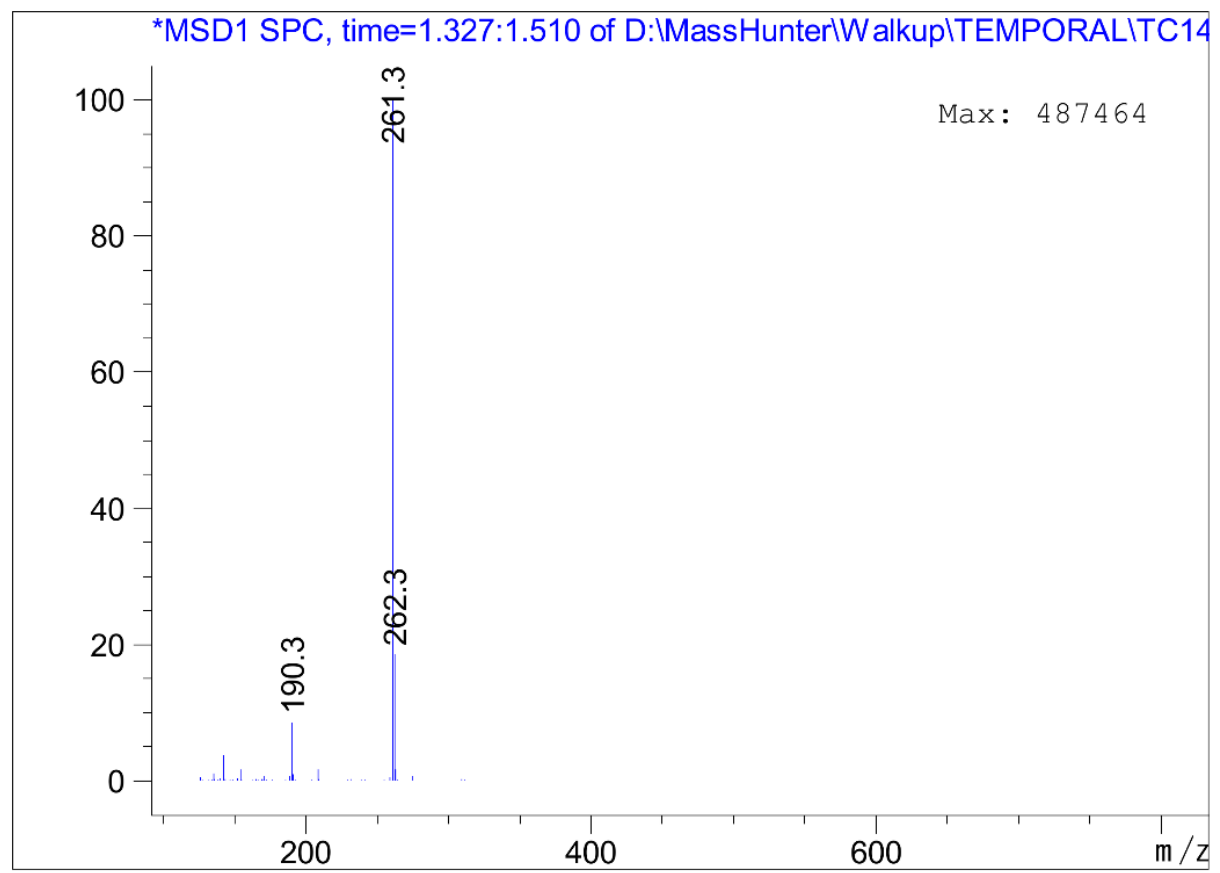


${ }^{1}$ H NMR (300 MHz, CDCl $)$ 10-benzyl-13-oxa-6,10-diazaspiro[4.1.5.$\left.^{7} 2^{5}\right]$ tetradecane $16\{3\}$
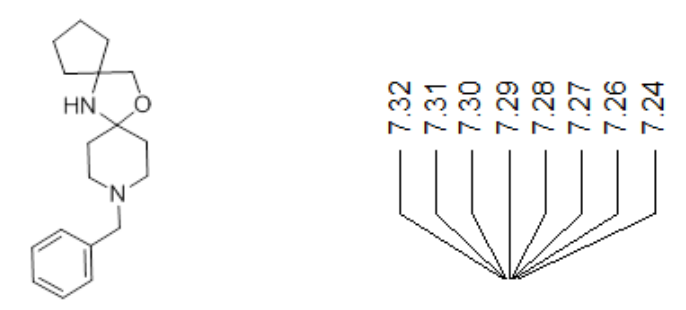

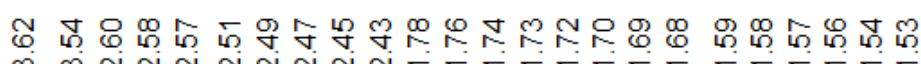
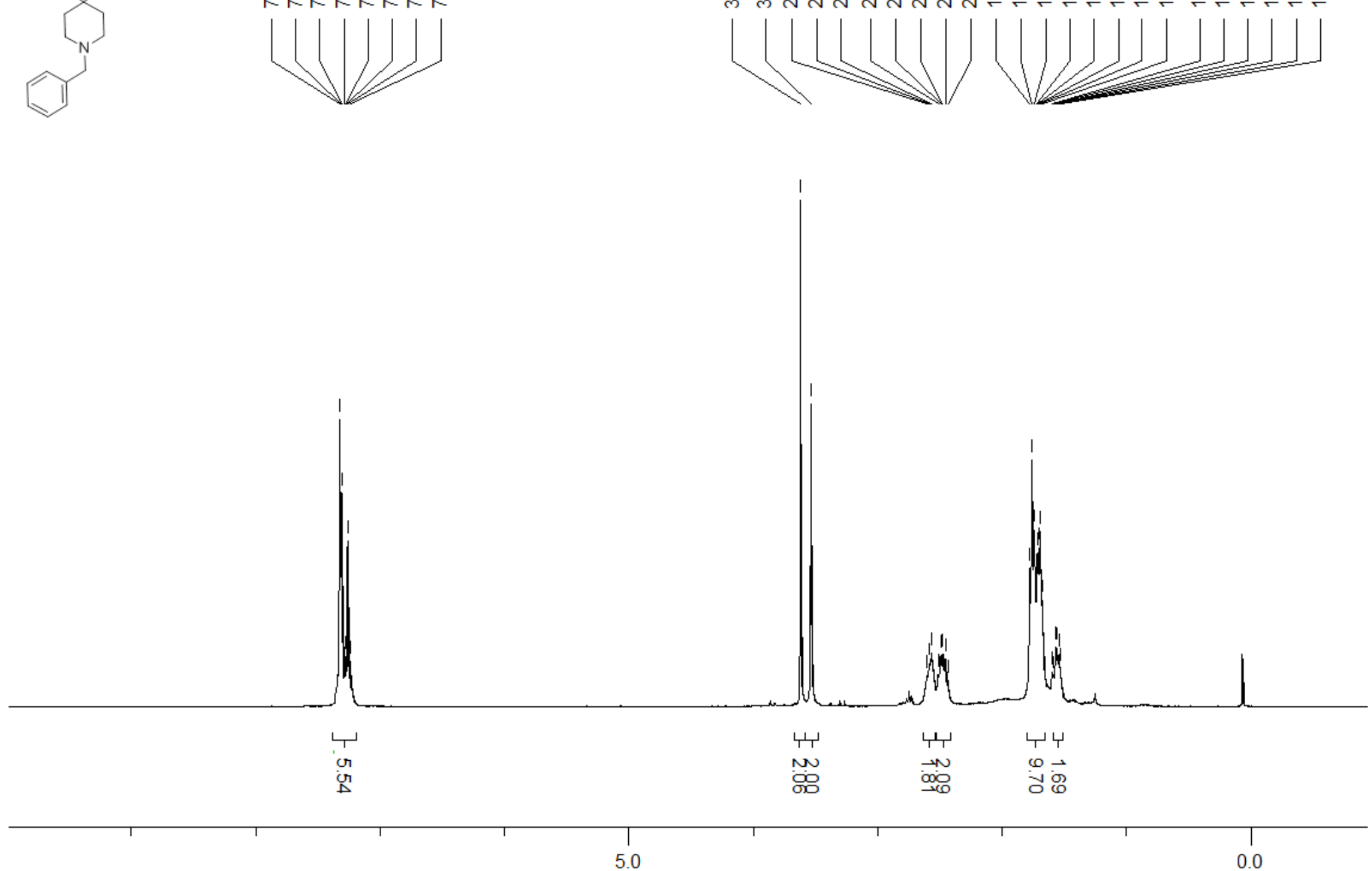

ppm (t1) 
${ }^{1} \mathrm{H}$ NMR (300 MHz, $\mathrm{CDCl}_{3}$ ) 3-benzyl-15-oxa-3,7-diazadispiro[5.1.5 ${ }^{8} \cdot 2^{6}$ pentadecane $16\{4\}$

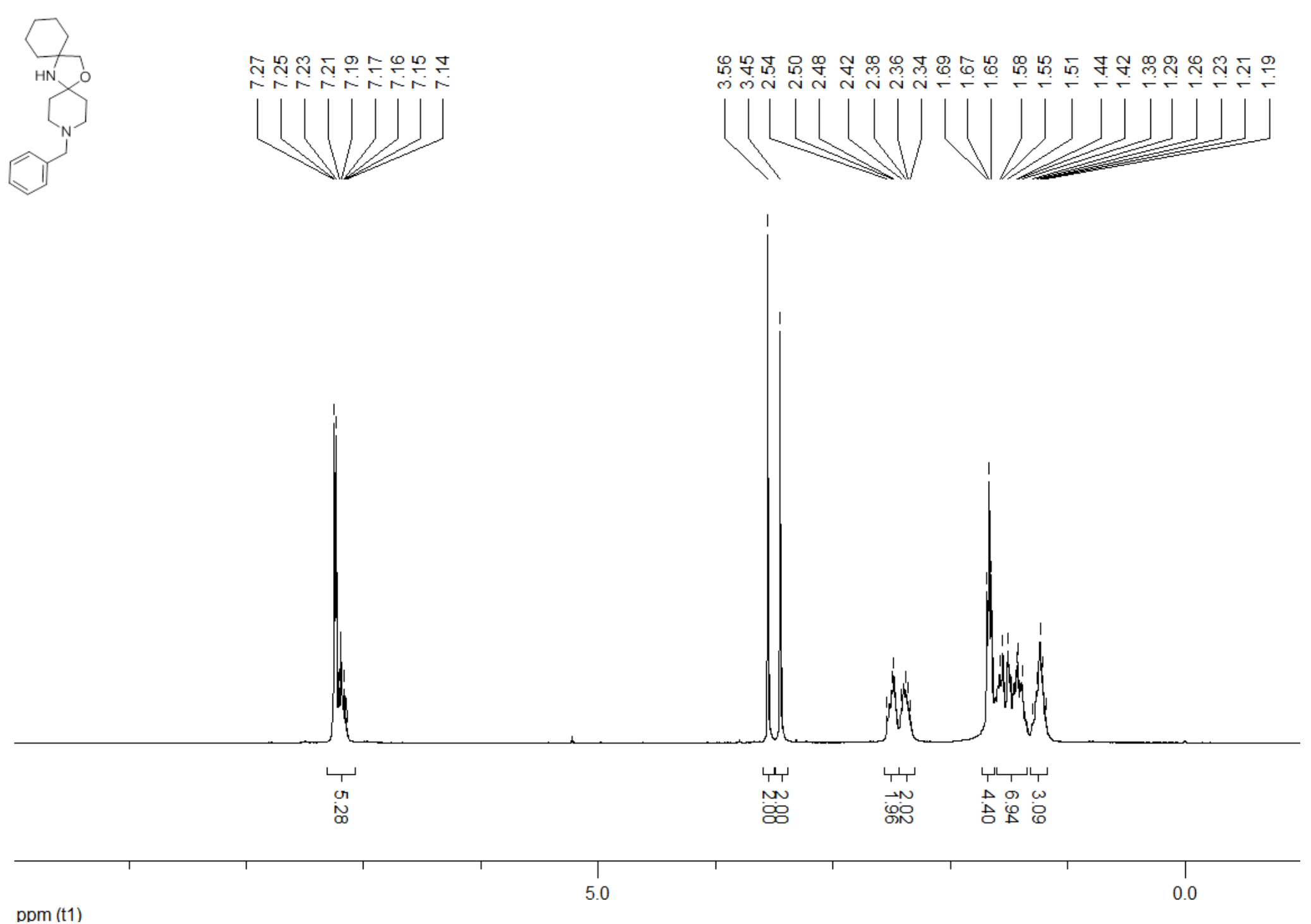


HPLC-MS (ESI) 3-benzyl-15-oxa-3,7-diazadispiro[5.1.5 $\left.{ }^{8} 2^{6}\right]$ pentadecane $16\{4\}$

\section{Retention Time : 1.837 minutes}
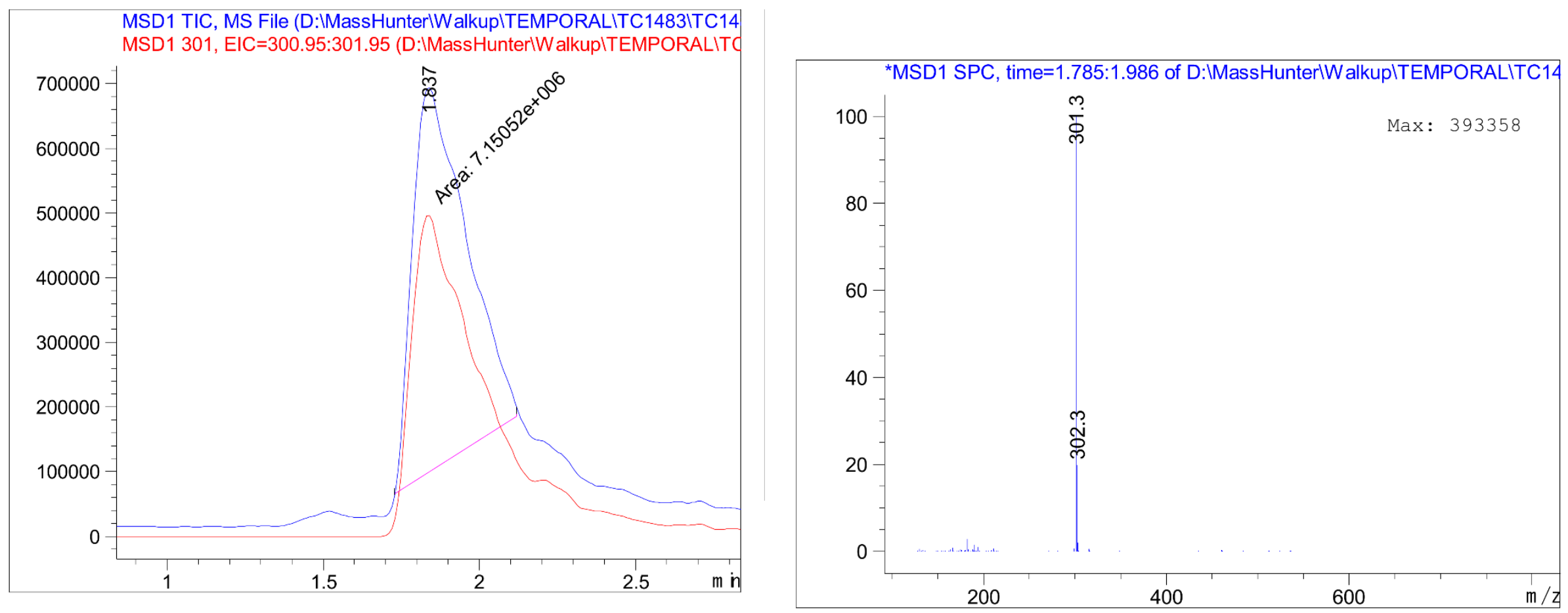
${ }^{1} \mathrm{H}$ NMR (300 MHz, CDCl 3 ) 11-benzyl-3,14-dioxa-7,11-diazadispiro[5.1.5 ${ }^{8} .2^{6}$ ]pentadecane $16\{5\}$

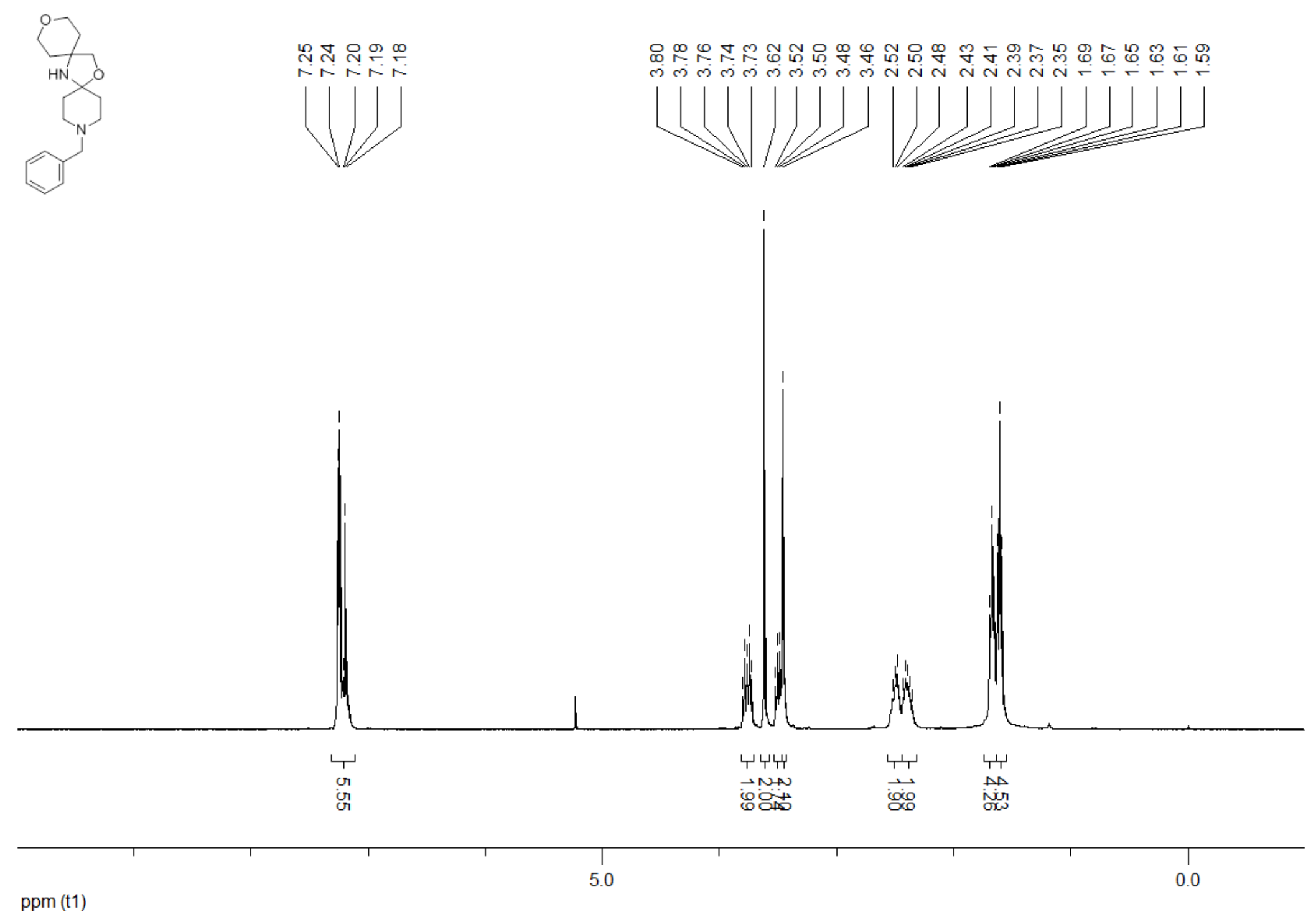


${ }^{1}$ H NMR (300 MHz, CDCl $)$ 1-\{8-benzyl-3,3-dimethyl-1-oxa-4,8-diazaspiro[4.5]decan-4-yl\}ethan-1-one 17\{1\}

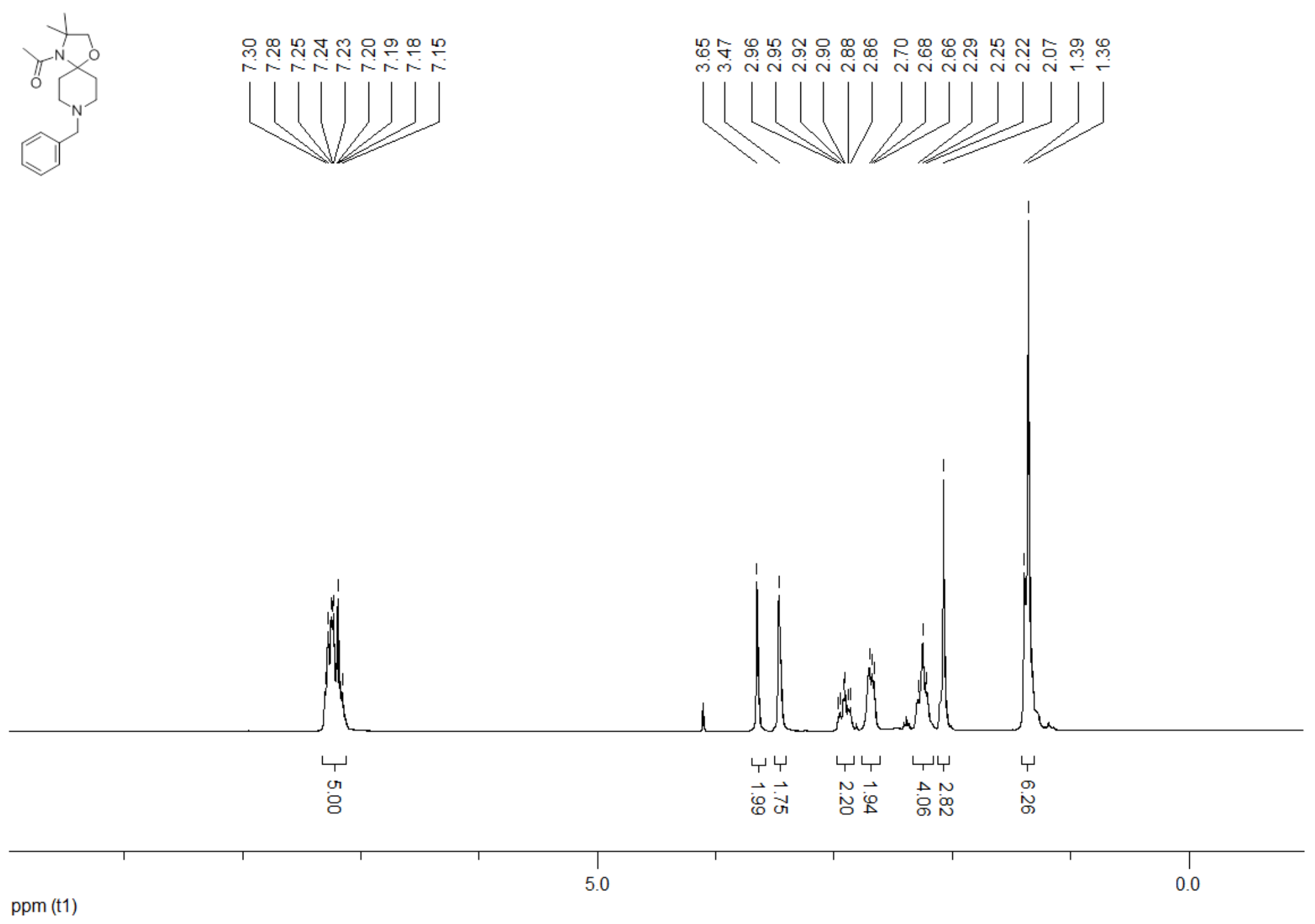


${ }^{1} \mathrm{H}$ NMR (300 MHz, CDCl $)$ 1-\{10-benzyl-13-oxa-6,10-diazadispiro[4.1.5 $\left.{ }^{7} .2^{5}\right]$ tetradecan-6-yl $\}$ ethan-1-one 17\{3\}
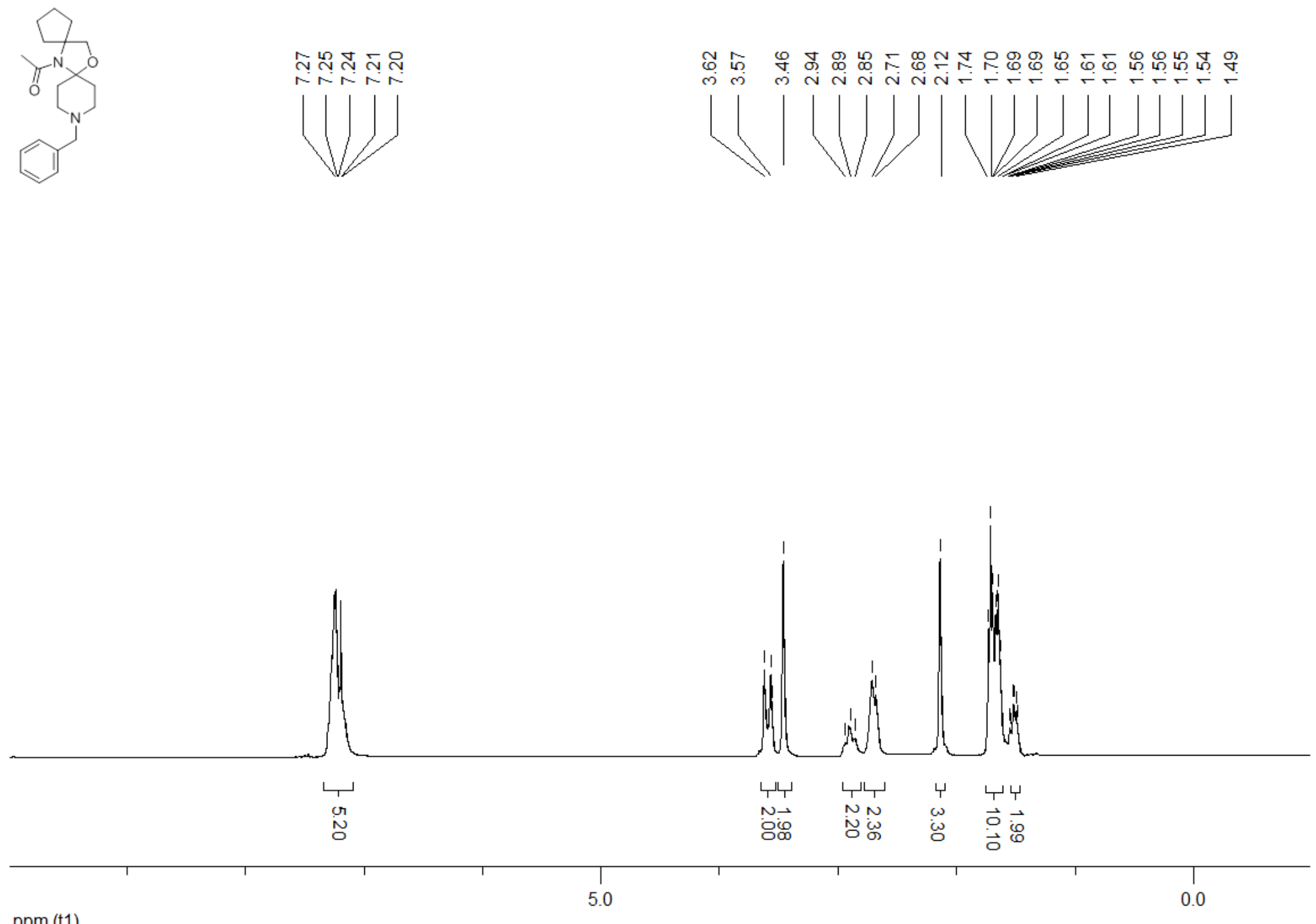

ppm (t1) 
HPLC-MS (ESI) 1-\{10-benzyl-13-oxa-6,10-diazadispiro[4.1.5 $\left.5^{7}\right]$ tetradecan-6-yl\} ethan-1-one 17\{3\}

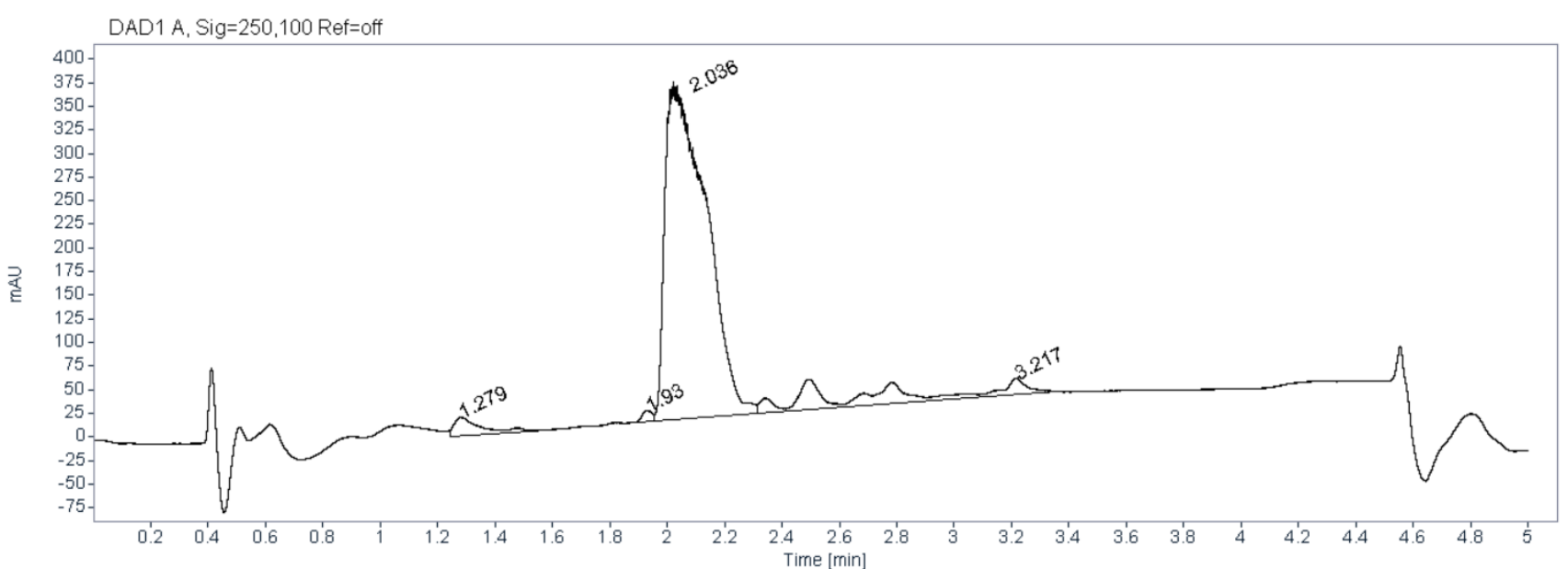

MSD1 SPC, MM-ES+APCI

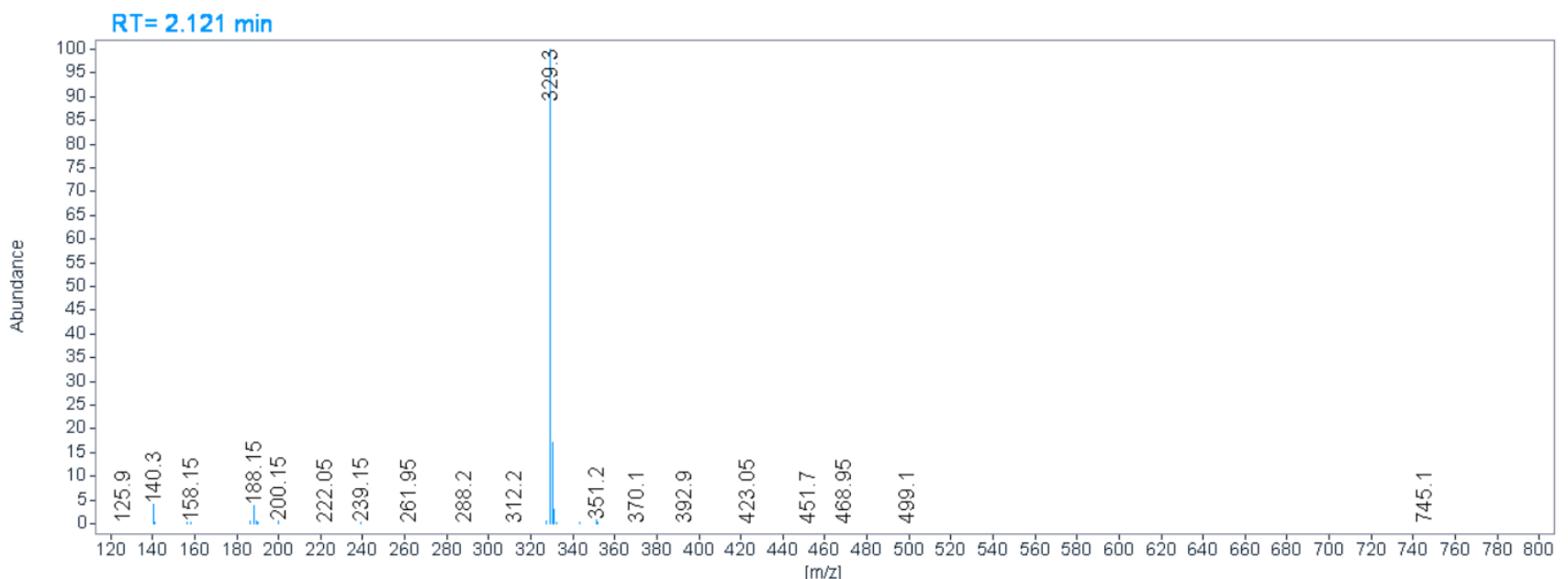


${ }^{1} \mathrm{H}$ NMR (300 MHz, $\mathrm{CDCl}_{3}$ ) 1-\{11-benzyl-3,14-dioxa-7,11-diazadispiro[5.1.5 $\left.{ }^{8} 2^{6}\right]$ pentadecan-7-yl\}ethan-1-one 17\{5\}

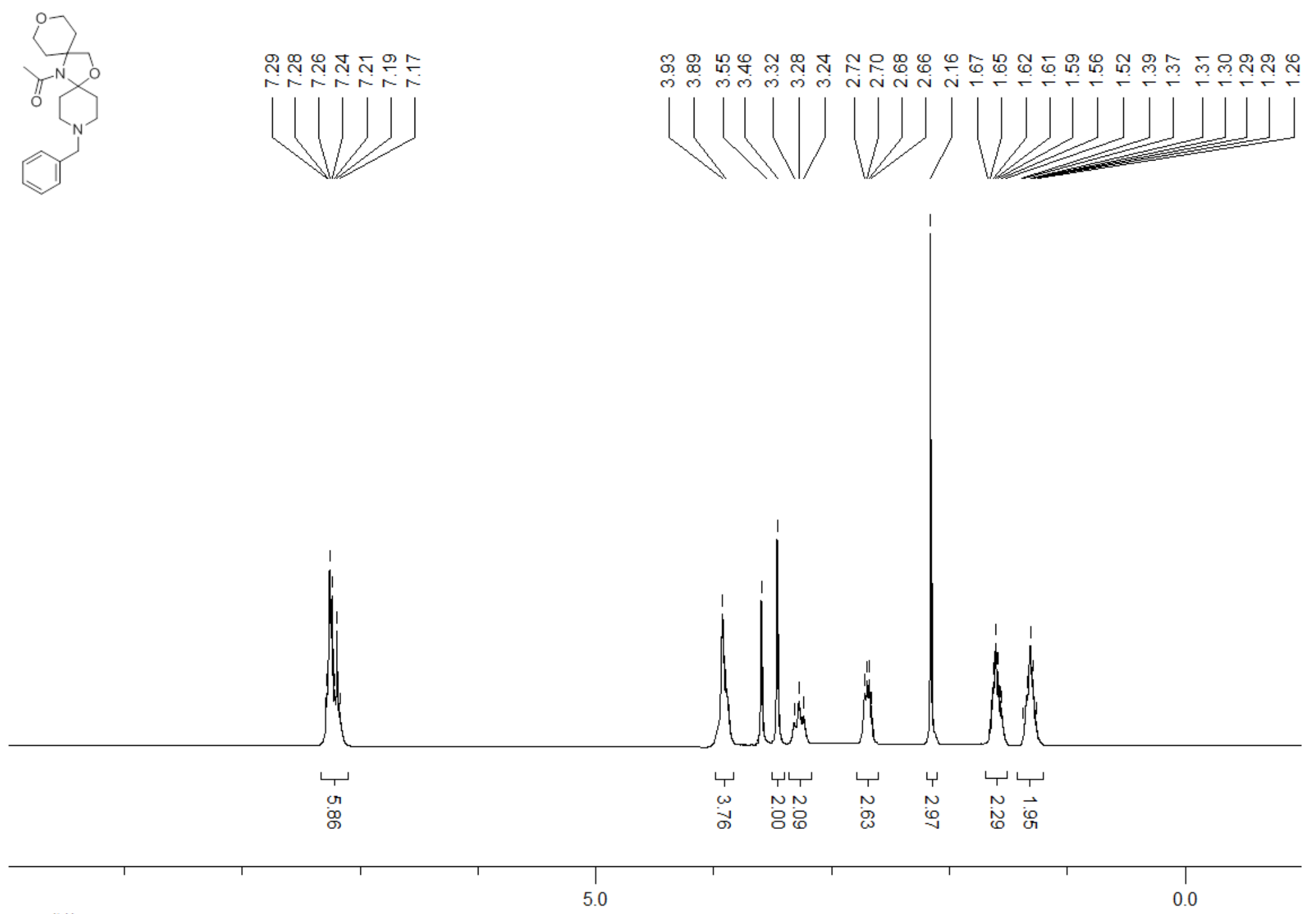

$\mathrm{ppm}(\mathrm{t} 1)$ 
HPLC-MS (ESI) 1-\{11-benzyl-3,14-dioxa-7,11-diazadispiro[5.1.5.$\left.^{8} 2^{6}\right]$ entadecan-7-yl\} ethan-1-one $17\{5\}$

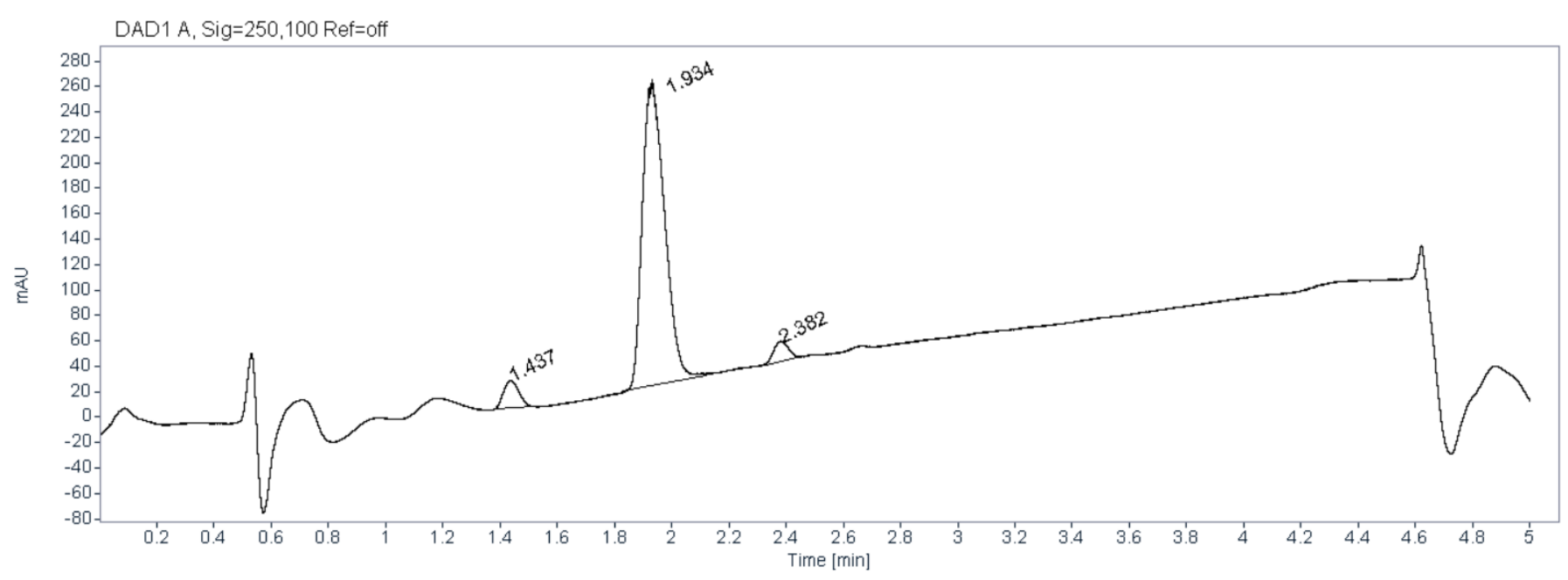

MSD1 SPC, MM-ES+APCI

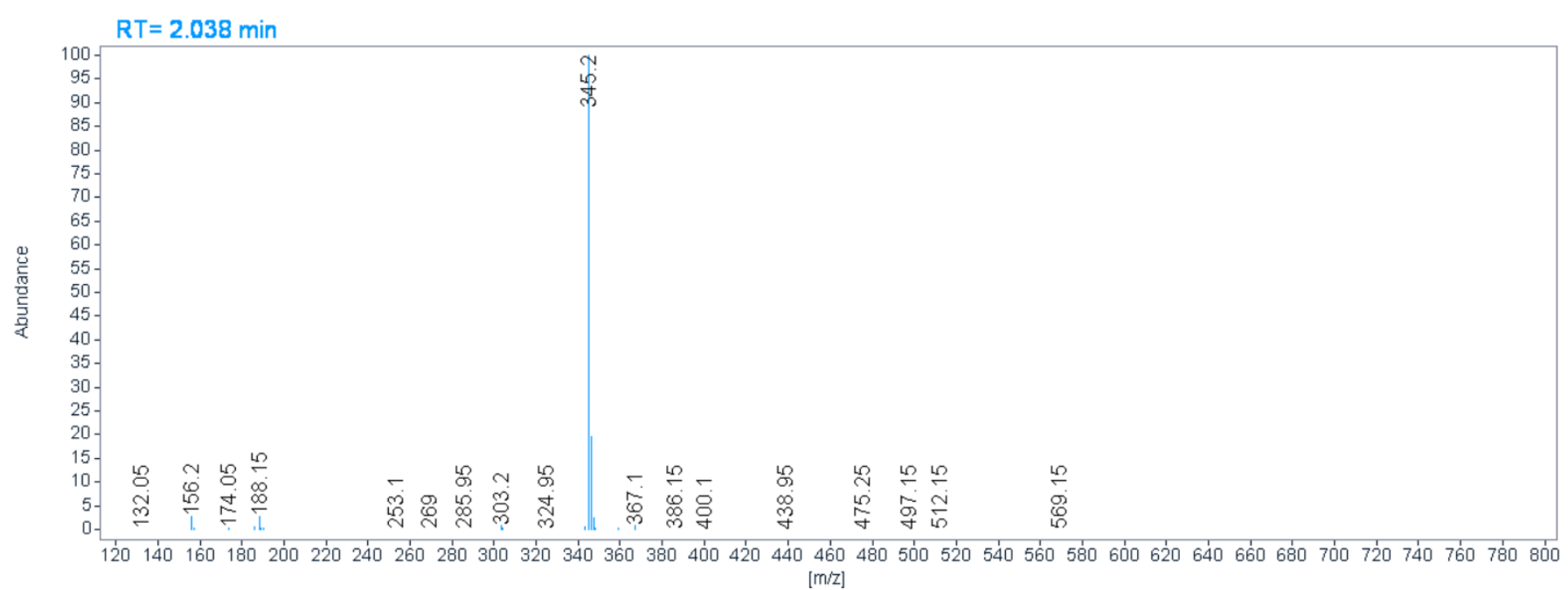


${ }^{1}$ H NMR (300 MHz, CDCl ${ }_{3}$ ) 4-benzoyl-8-benzyl-3,3-dimethyl-1-oxa-4,8-diazaspiro[4.5]decane $17\{8\}$

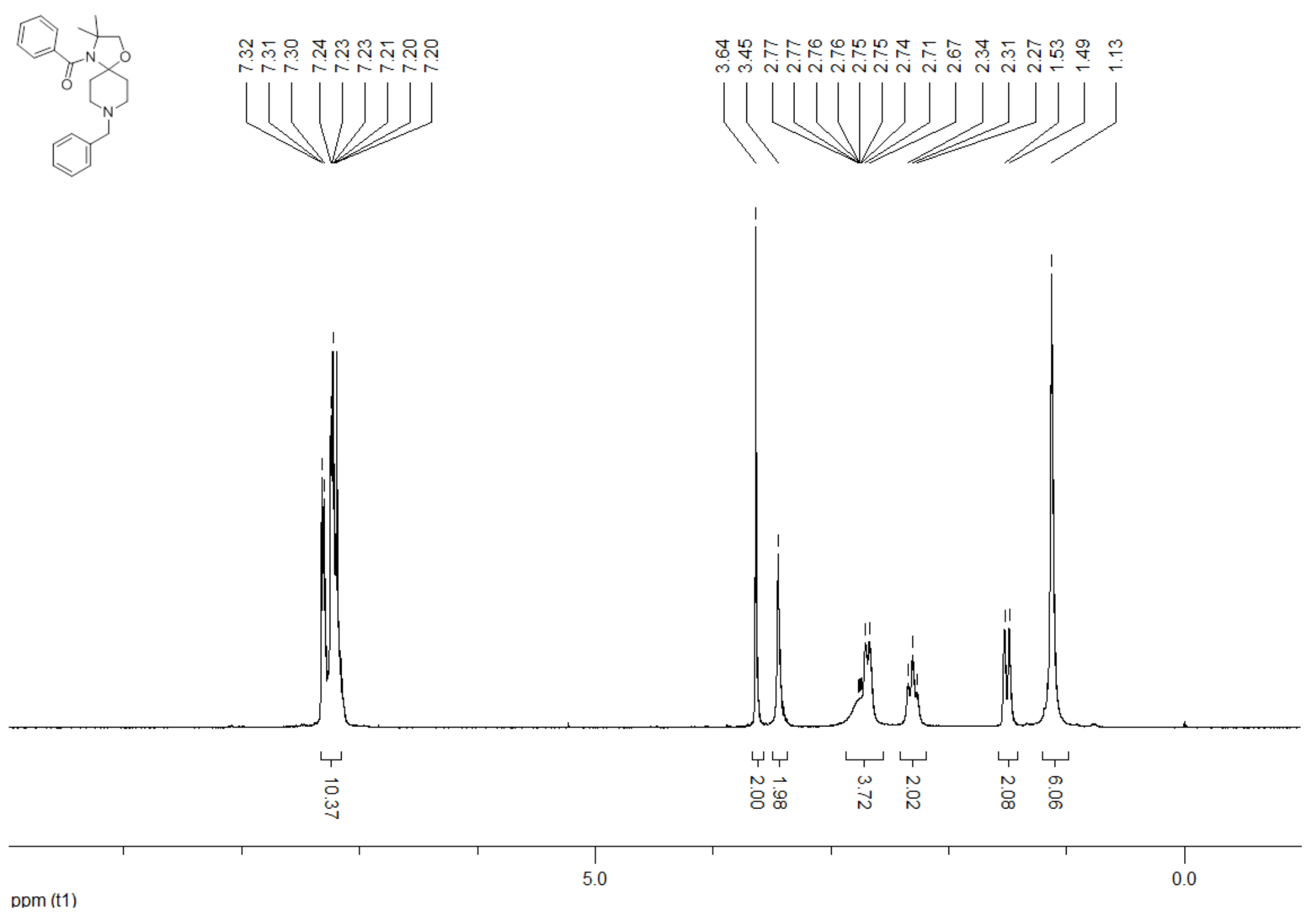


${ }^{1}$ H NMR (300 MHz, CDCl $)$ ) 1-\{3,3-dimethyl-1-oxa-4,8-diazaspiro[4.5]decan-4-yl\}ethan-1-one 18\{1\}
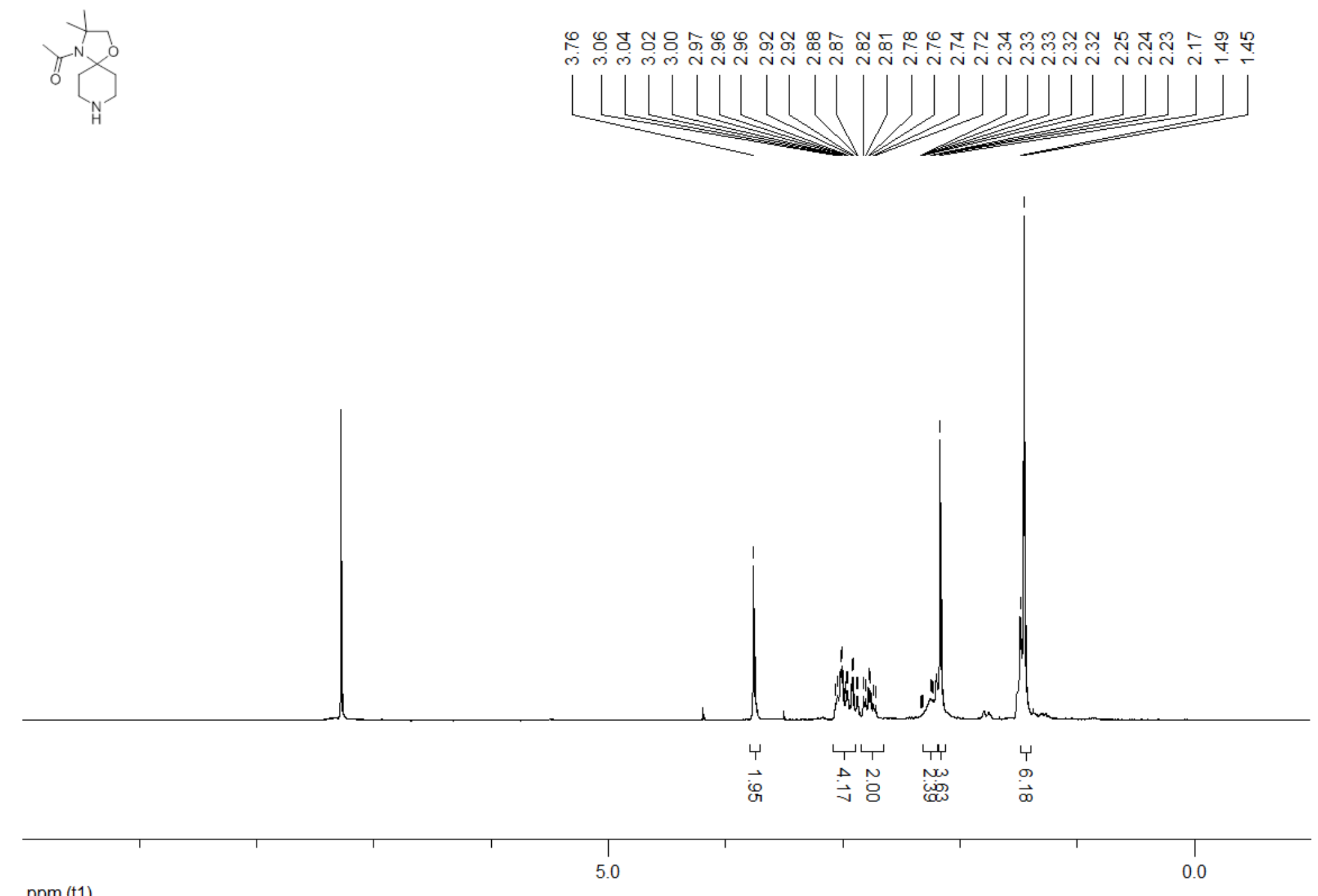

ppm (t1) 
HPLC-MS (ESI) 1-\{3,3-dimethyl-1-oxa-4,8-diazaspiro[4.5]decan-4-yl\}ethan-1-one $18\{1\}$

\section{Retention Time : 1.455 minutes}

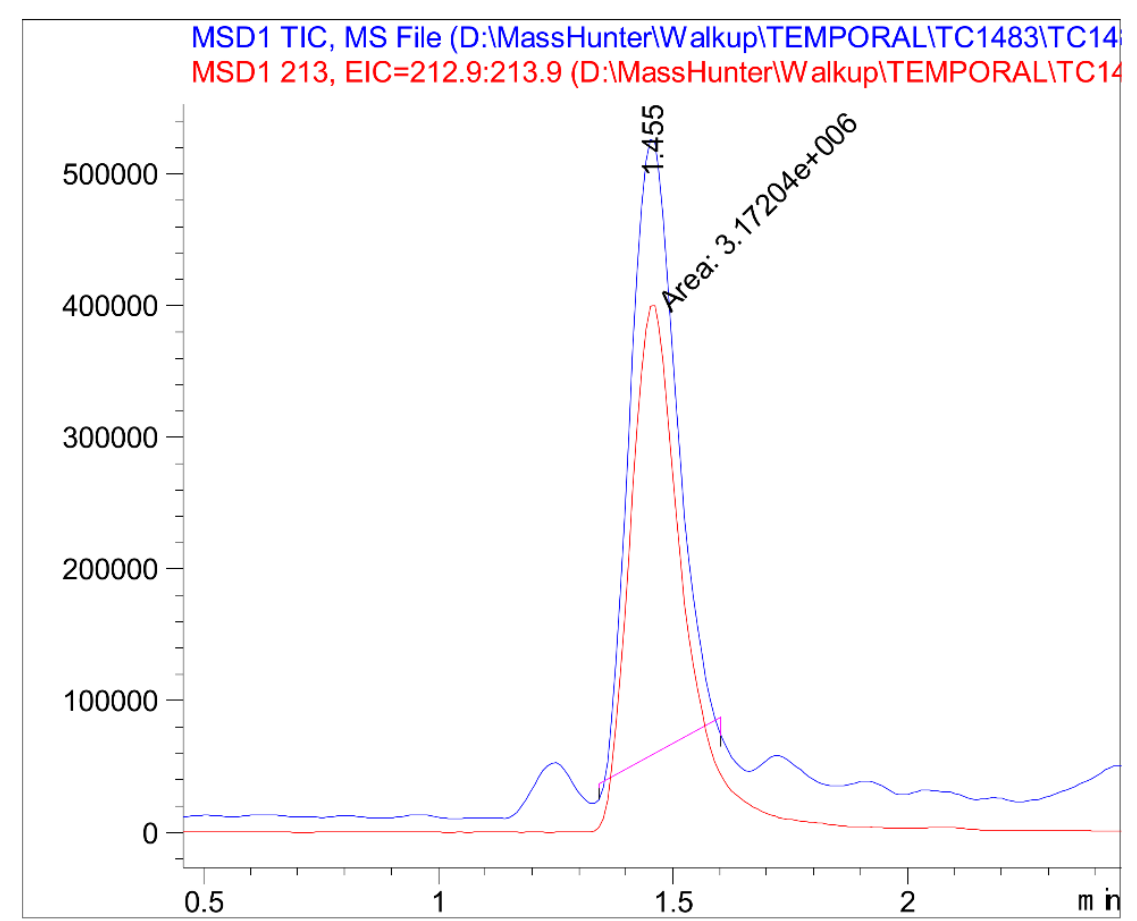

*MSD1 SPC, time=1.406:1.507 of D:IMassHunterlWalkupITEMPORALITC14

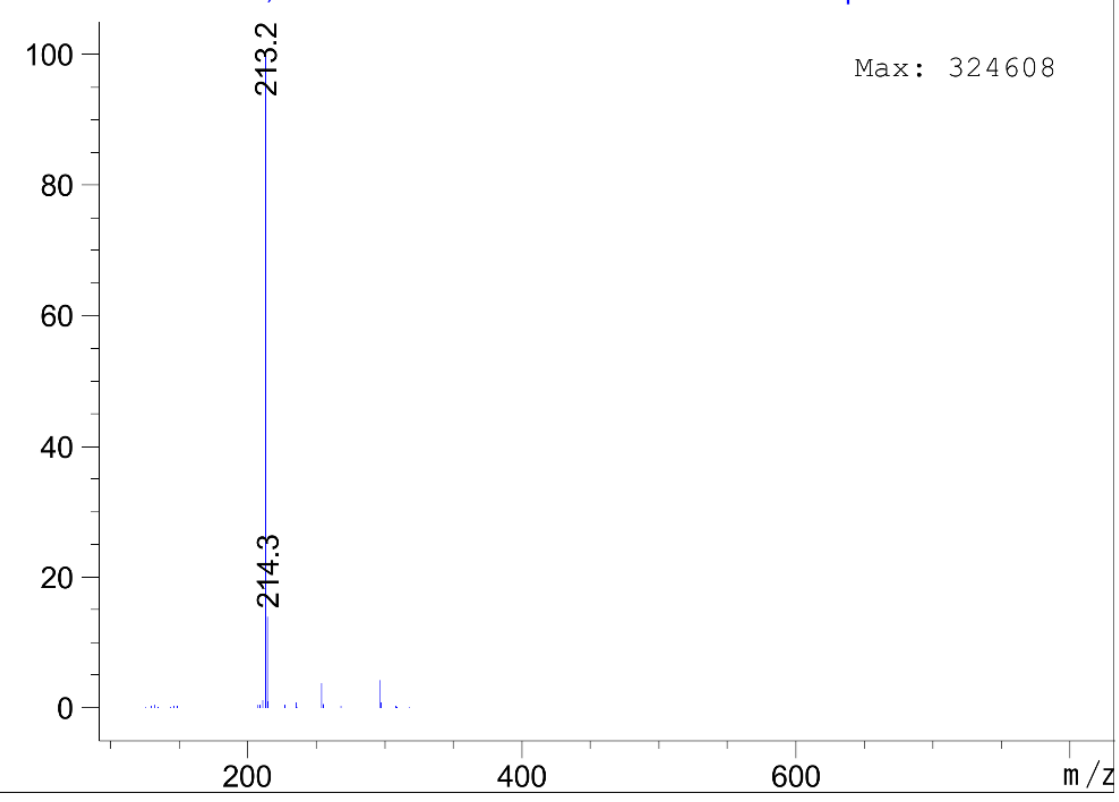


${ }^{1} \mathrm{H}$ NMR (300 MHz, CDCl $)$ ) 1-\{13-oxa-6,10-diazadispiro[4.1.5 $\left..^{7} .^{5}\right]$ tetradecan-6-yl\}ethan-1-one 18 $\{3\}$

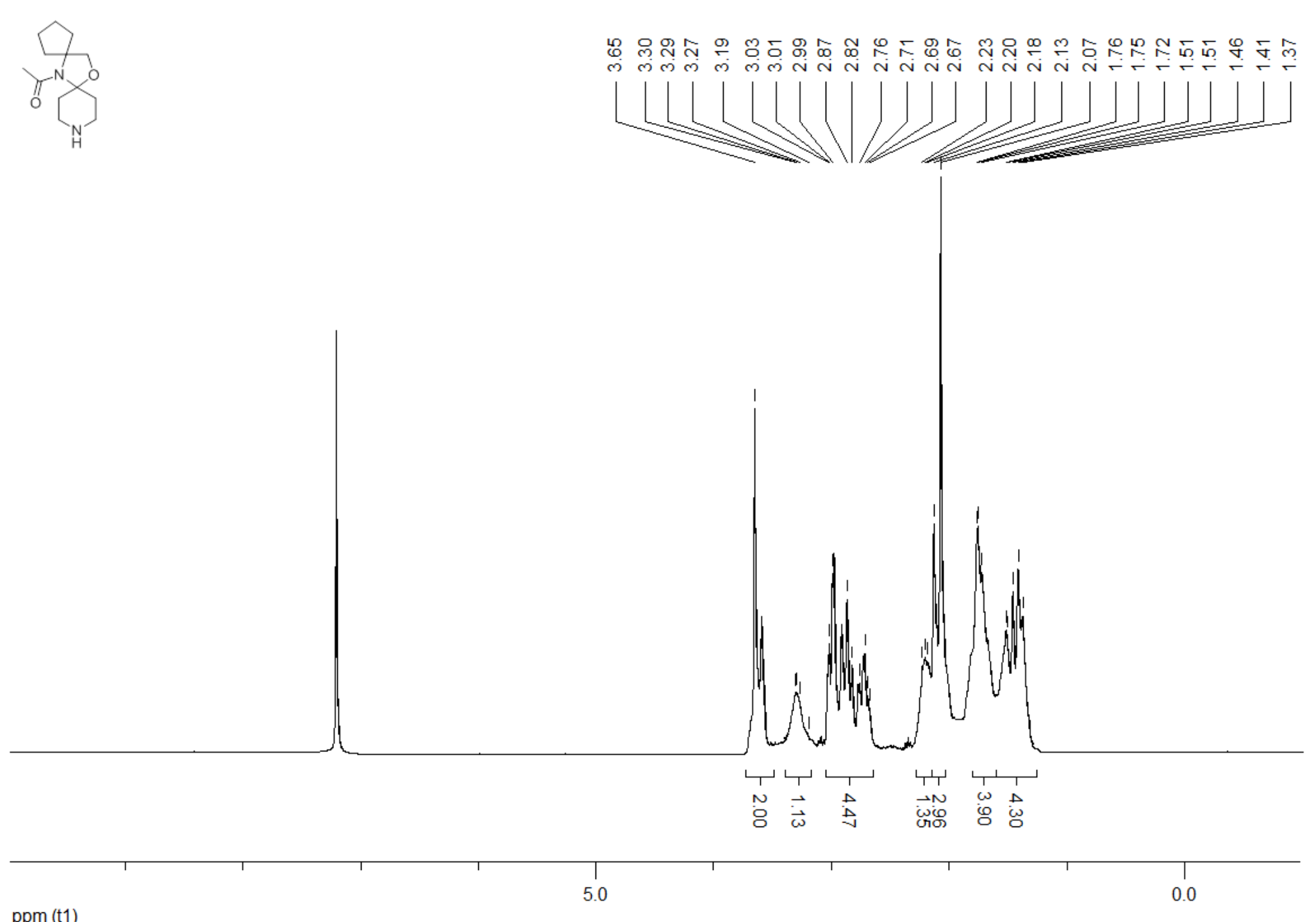

ppm (t1) 
HPLC-MS (ESI) 1-\{13-oxa-6,10-diazadispiro[4.1.5 $\left..^{7} .^{5}\right]$ tetradecan-6-yl\}ethan-1-one 18 $\{3\}$

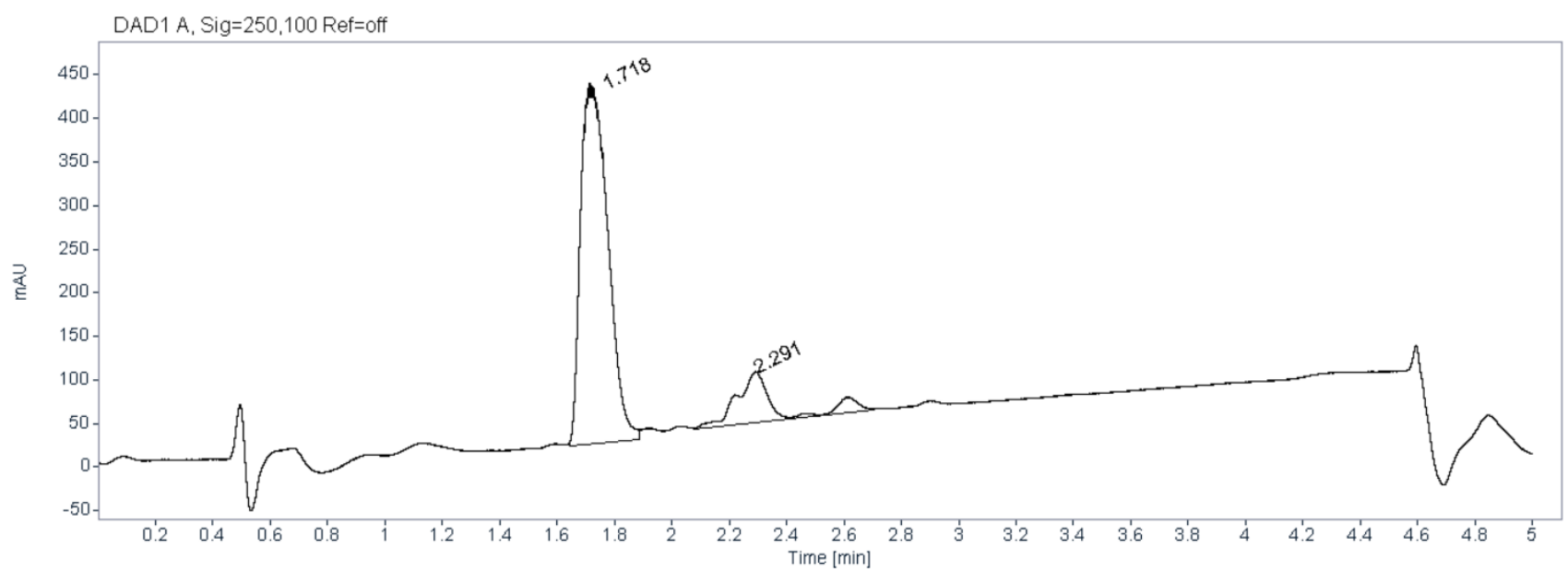

MSD1 SPC, MM-ES+APCI

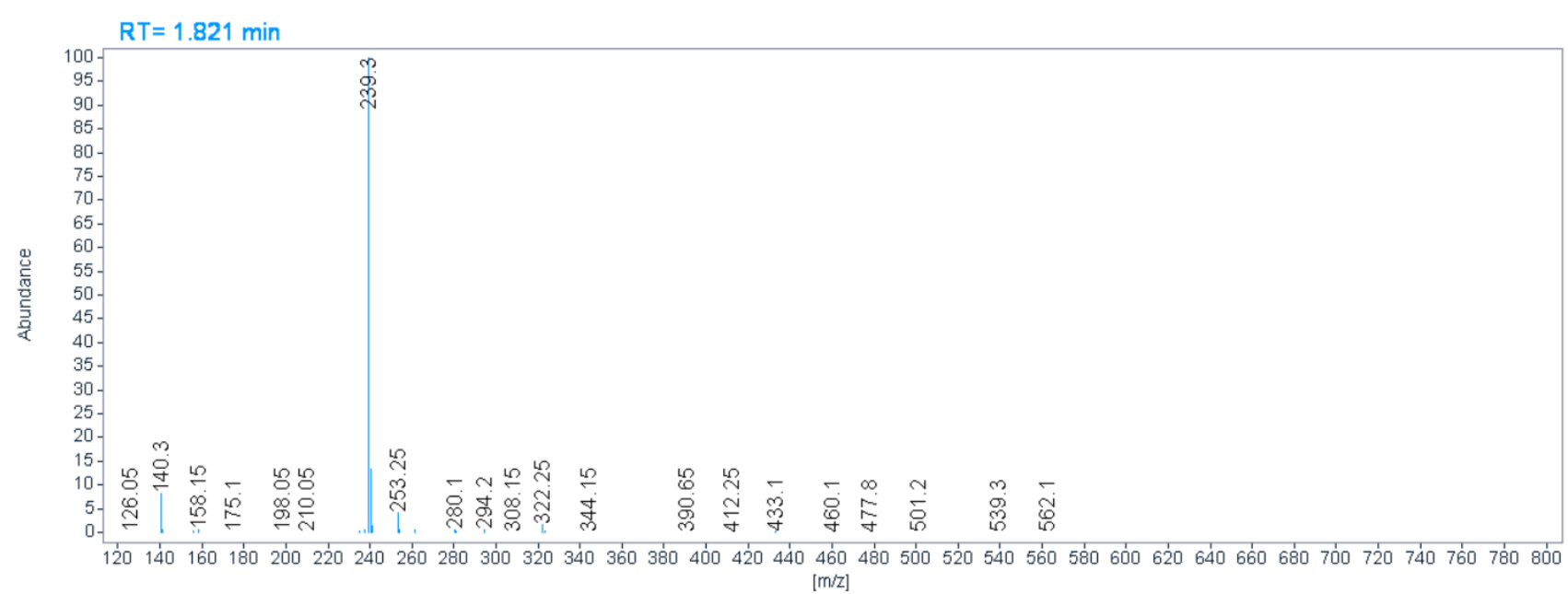

Signal: $\quad$ DAD1 A, Sig=250,100 Ref $=$ off 
${ }^{1} \mathrm{H}$ NMR (300 MHz, CDCl $)$ ) 1-\{15-oxa-3,7-diazadispiro[5.1. $\left.5^{8} .2^{6}\right]$ pentadecan-7-yl\}ethan-1-one 18 $\{4\}$
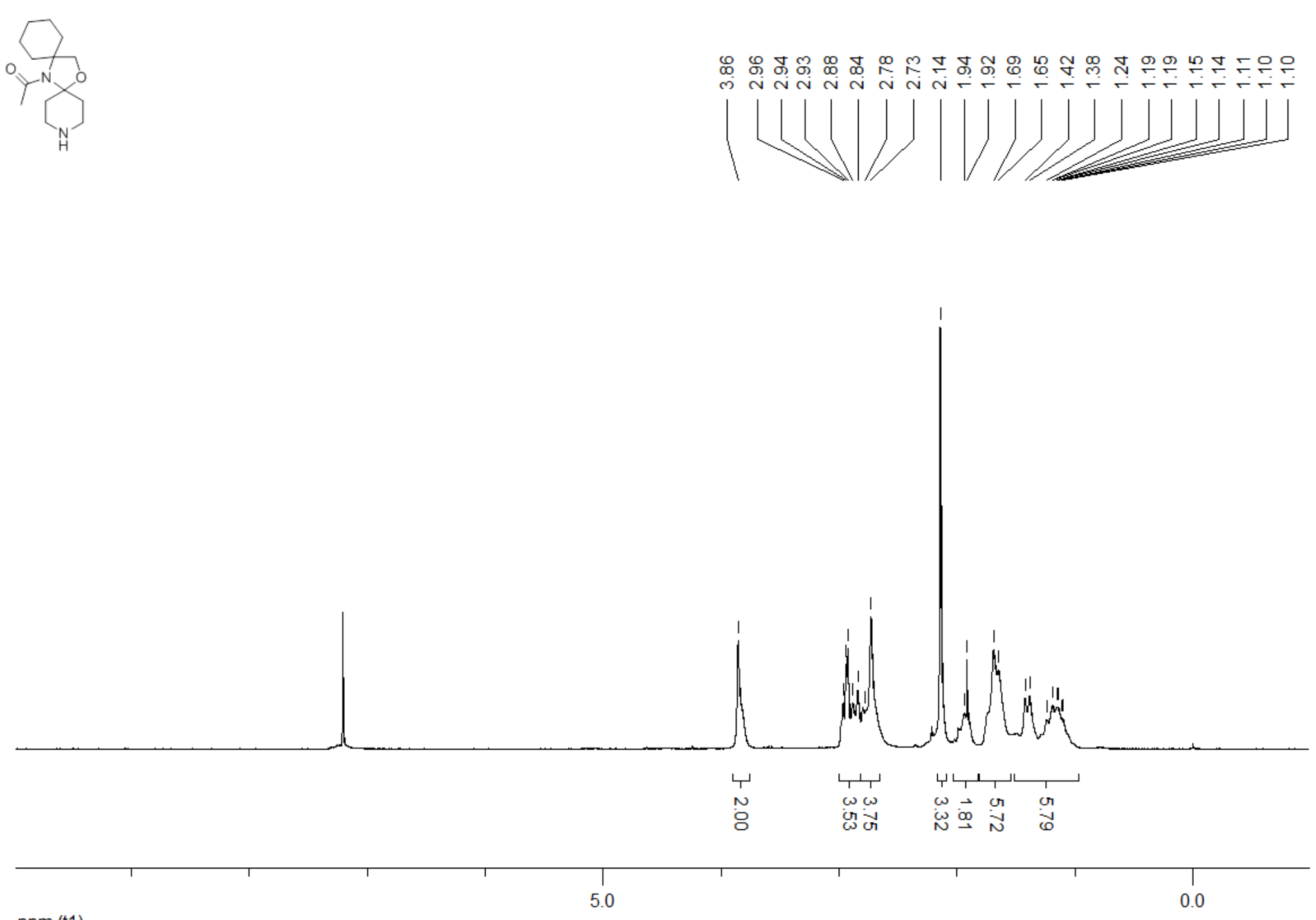

ppm (t1) 
${ }^{1} \mathrm{H}$ NMR (300 MHz, $\mathrm{CDCl}_{3}$ ) 4-benzoyl-3,3-dimethyl-1-oxa-4,8-diazaspiro[4.5]decane 18 $\{8\}$
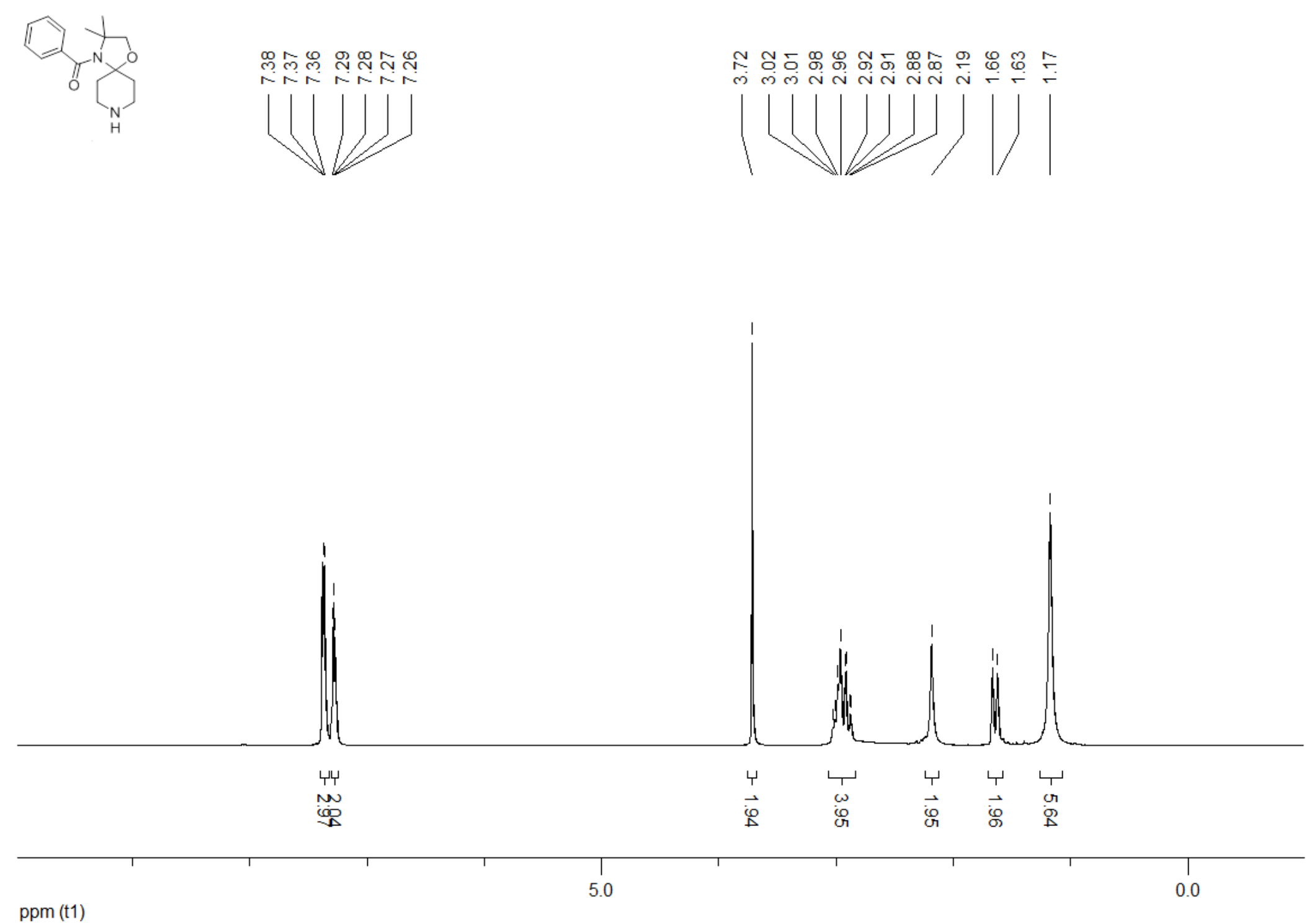
${ }^{1} \mathrm{H}$ NMR (300 MHz, CDCl $)$ 1-[10-(1-methyl-1H-pyrrole-2-carbonyl)-13-oxa-6,10-diazadispiro[4.1.5.$^{7}{ }^{5}$ ]tetradecan-6-yl]ethan-1-one 19\{3.1\}

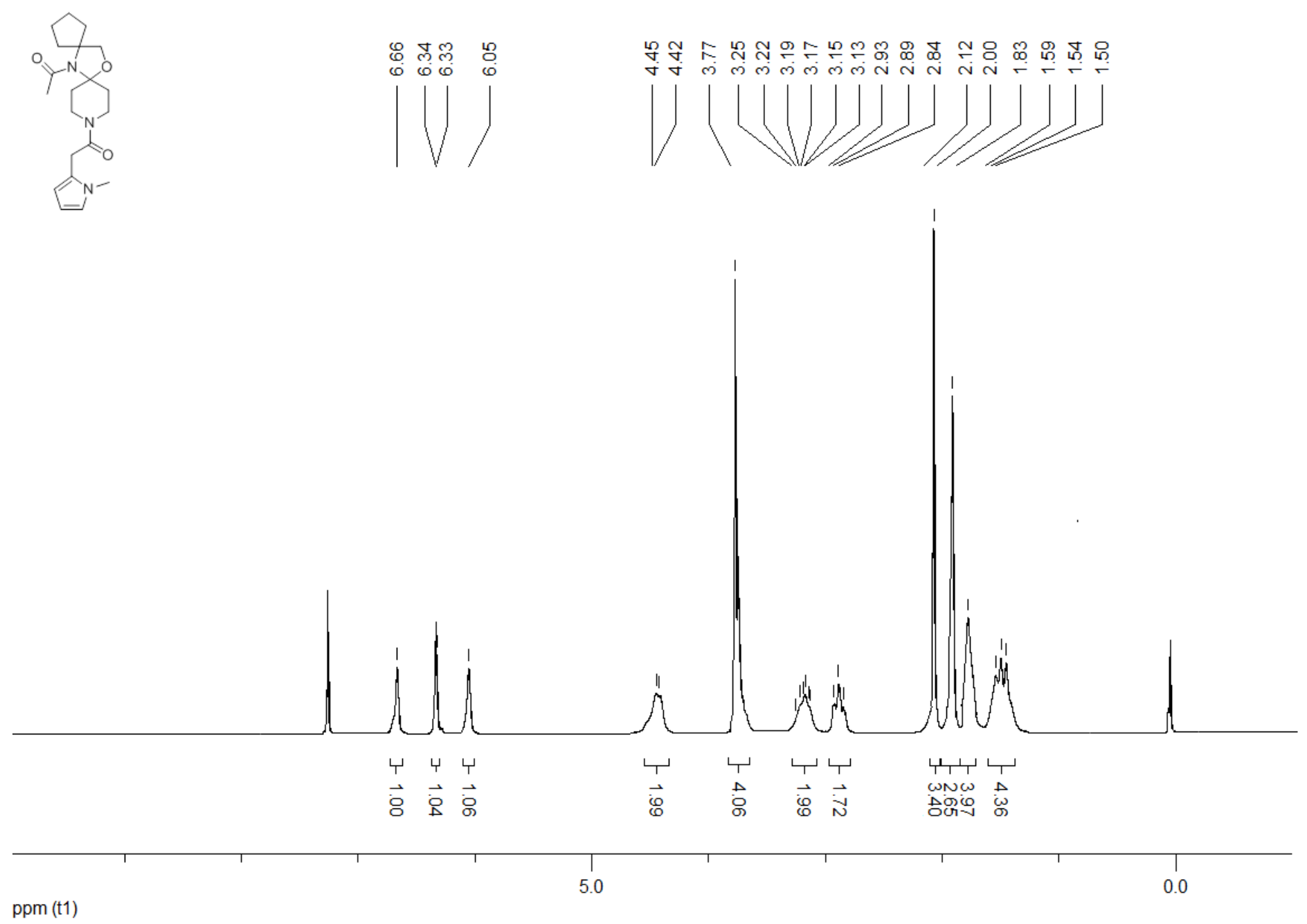


${ }^{1} \mathrm{H}$ NMR (300 MHz, $\mathrm{CDCl}_{3}$ ) 1-\{7-acetyl-15-oxa-3,7-diazadispiro[5.1.5 $\left.5^{8} .^{6}\right]$ pentadecan-3-yl\}-2-methylpropan-1-one 19\{4.1\}

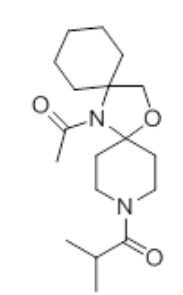

郘荌
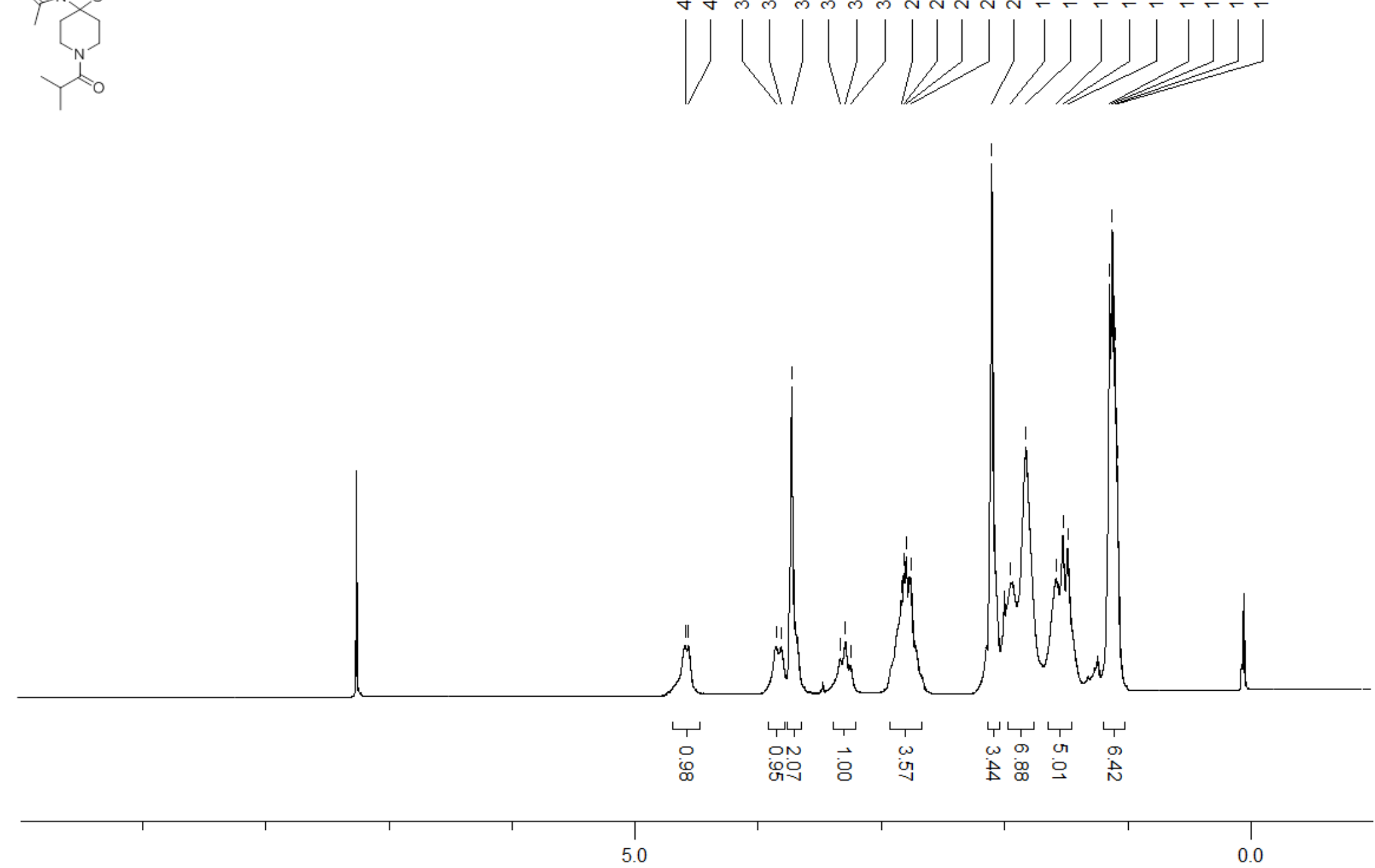

ppm (t1) 
${ }^{1}$ H NMR (300 MHz, CDCl $)$ 1-\{10-[(1H-imidazol-4-yl)methyl]-13-oxa-6,10-diazadispiro[4.1.5 $\left.{ }^{7} .2^{5}\right]$ tetradecan-6-yl\} ethan-1-one 20\{3.1\}
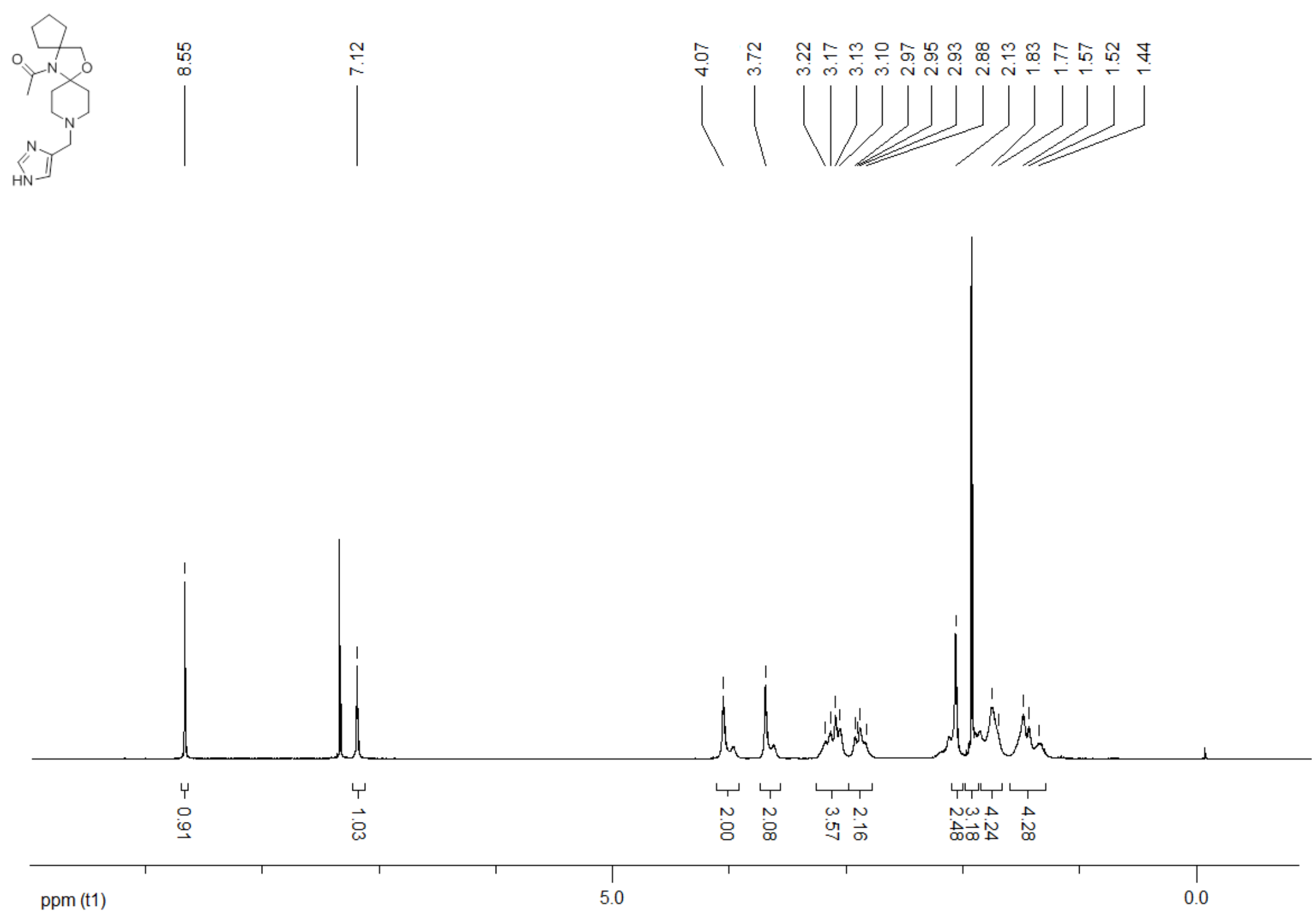
${ }^{1}$ H NMR (300 MHz, CDCl $)$ 1-\{11-[(2-chloro-6-fluorophenyl)methyl]-3,14-dioxa-7,11-diazadispiro[5.1. $\left.5^{8} .2^{6}\right]$ pentadecan-7-yl\}ethan-1one $20\{5.1\}$
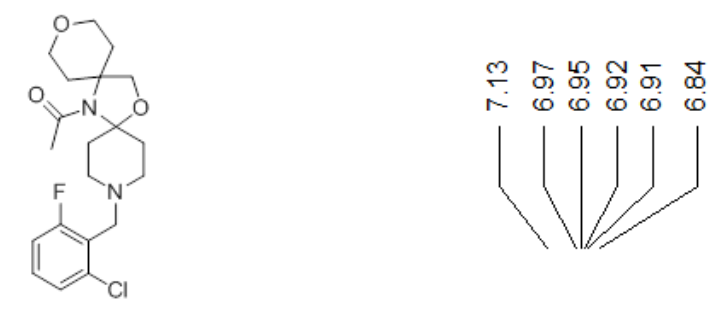

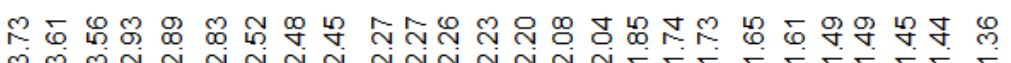
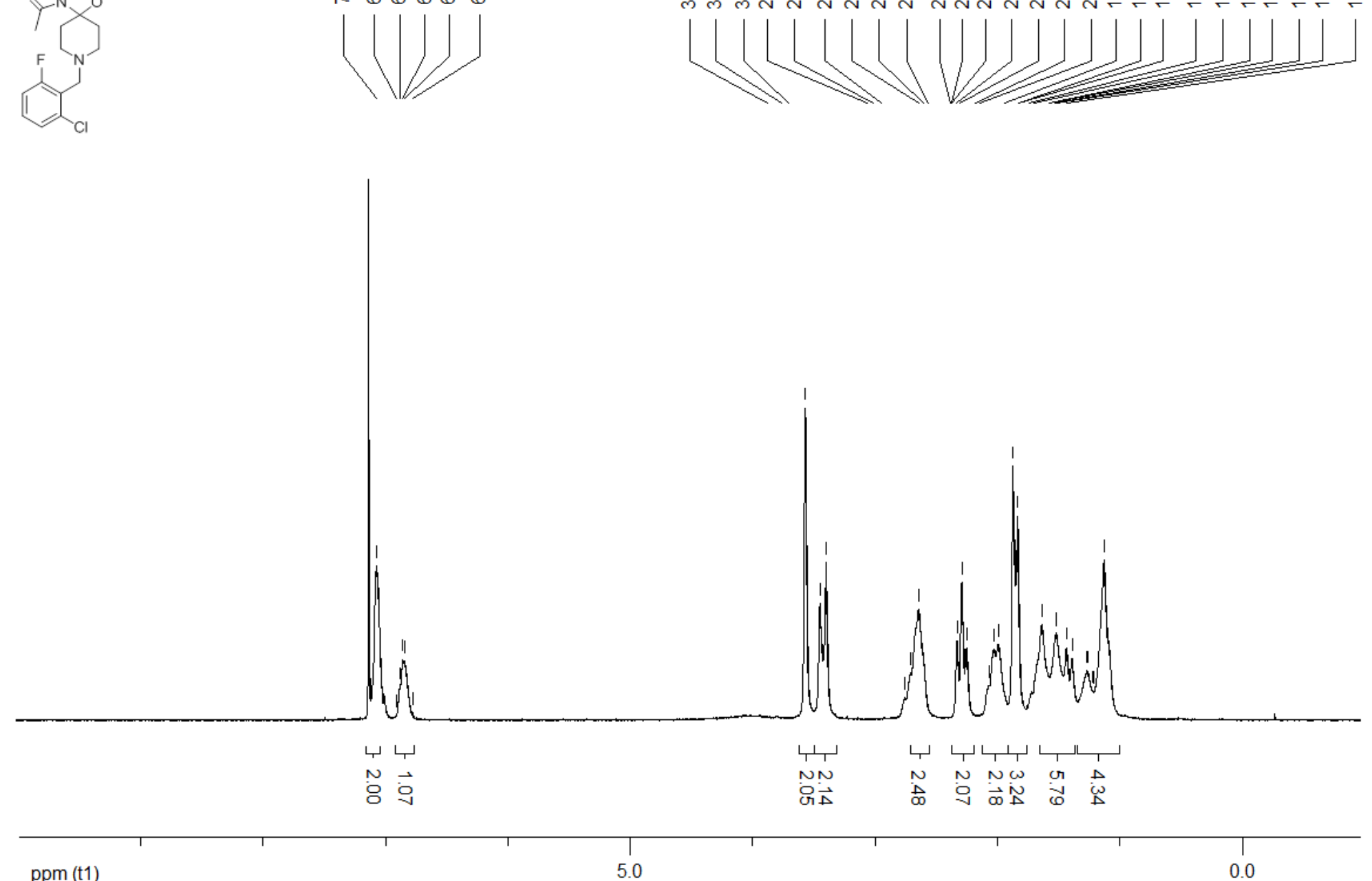
${ }^{1}$ H NMR (300 MHz, CDCl $)$ 1-[10-(2,5-dimethoxybenzenesulfonyl)-13-oxa-6,10-diazadispiro[4.1.5 $\left.{ }^{7} .2^{5}\right]$ tetradecan-6-yl]ethan-1-one 21\{3.1\}

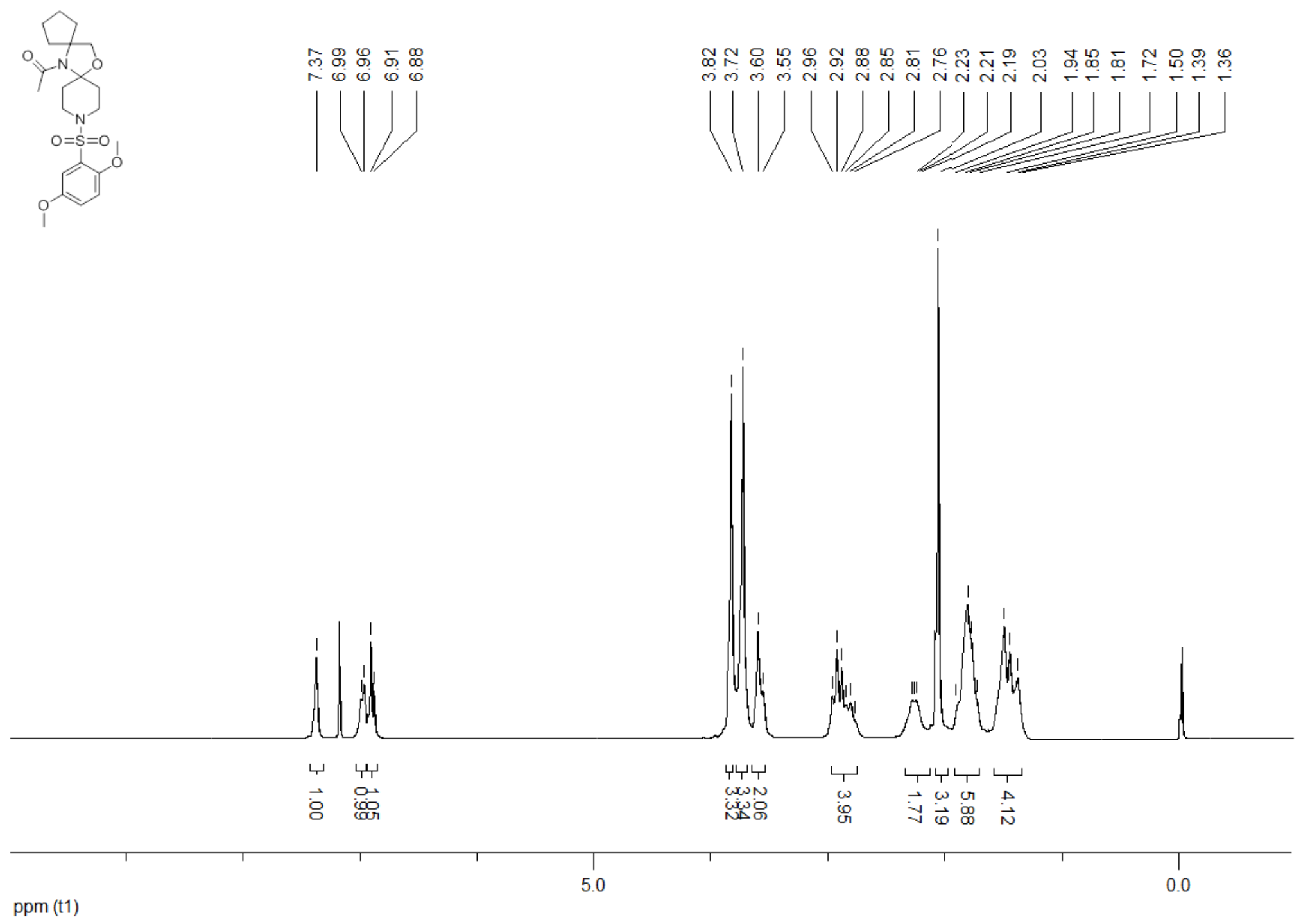


HPLC-MS (ESI) 1-[10-(2,5-dimethoxybenzenesulfonyl)-13-oxa-6,10-diazadispiro[4.1.5 $\left.{ }^{7} 2^{5}\right]$ tetradecan-6-yl]ethan-1-one 21\{3.1\}

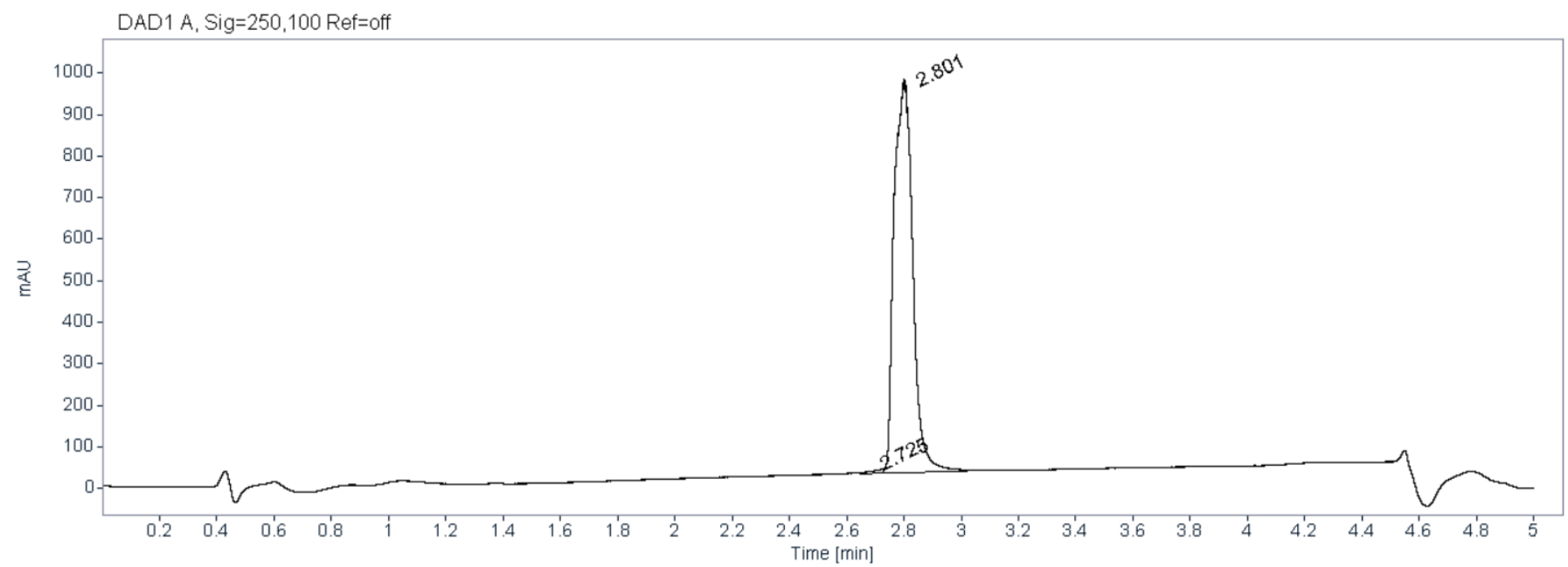

MSD1 SPC, MM-ES+APCI

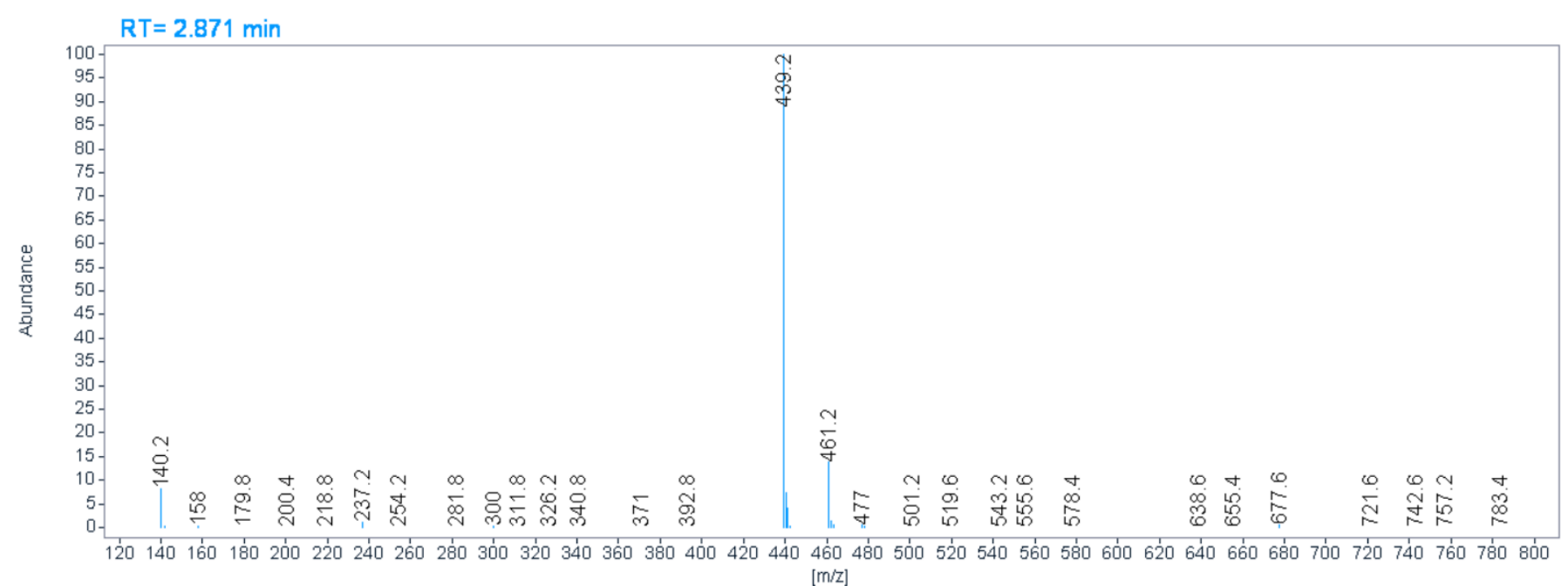


${ }^{1} \mathrm{H}$ NMR (300 MHz, $\mathrm{CDCl}_{3}$ ) 1-\{3-methanesulfonyl-15-oxa-3,7-diazadispiro[5.1.5 ${ }^{8} .2^{6}$ ]pentadecan-7-yl\}ethan-1-one 21 $\{4.1\}$
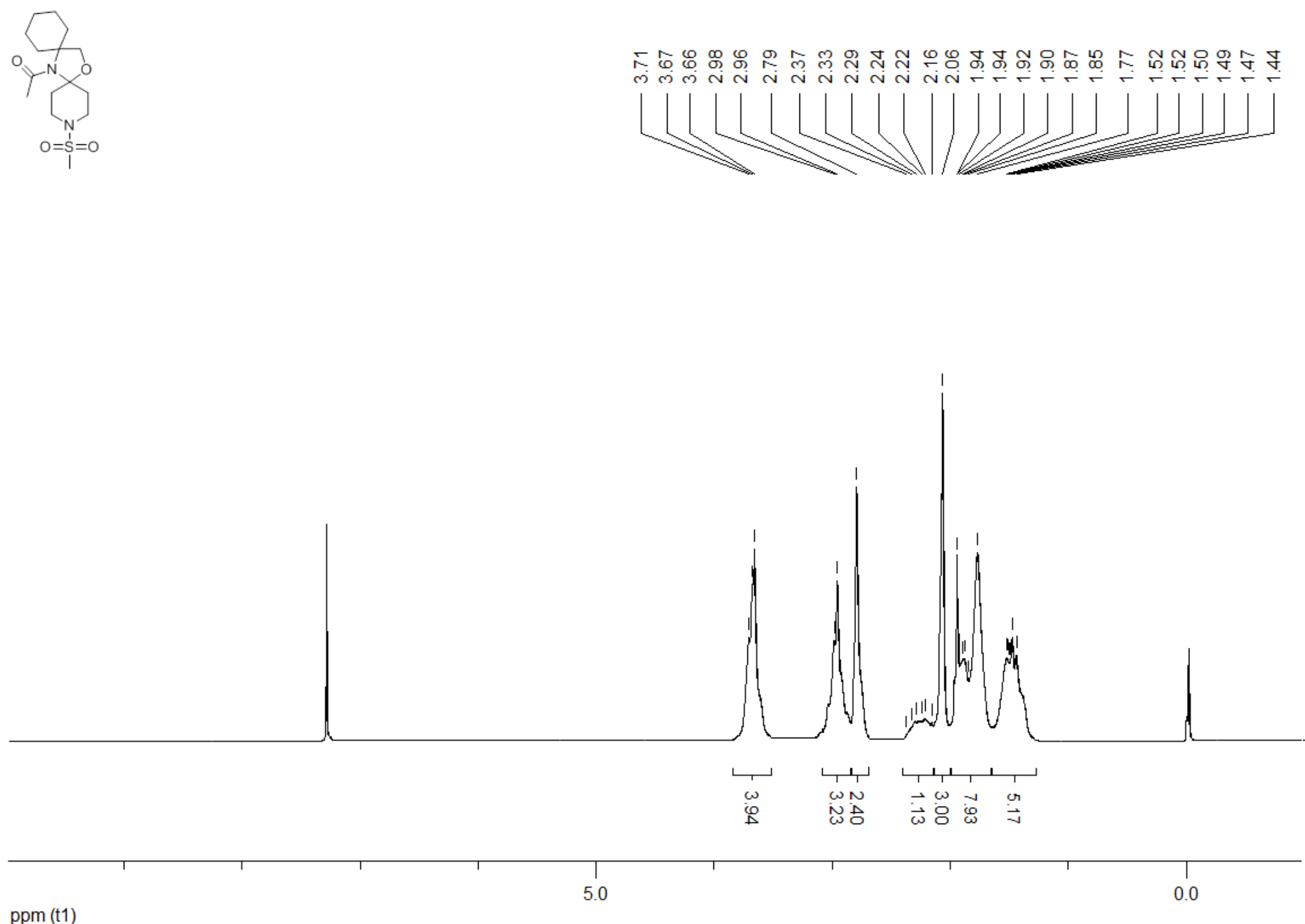

ppm (t1) 
${ }^{1} \mathrm{H}$ NMR (300 MHz, CDCl 3 ) 6-acetyl-N-(2,6-dichlorophenyl)-13-oxa-6,10-diazadispiro[4.1.5.$\left.^{7}{ }^{5}\right]$ tetradecane-10-carboxamide $22\{3.1\}$
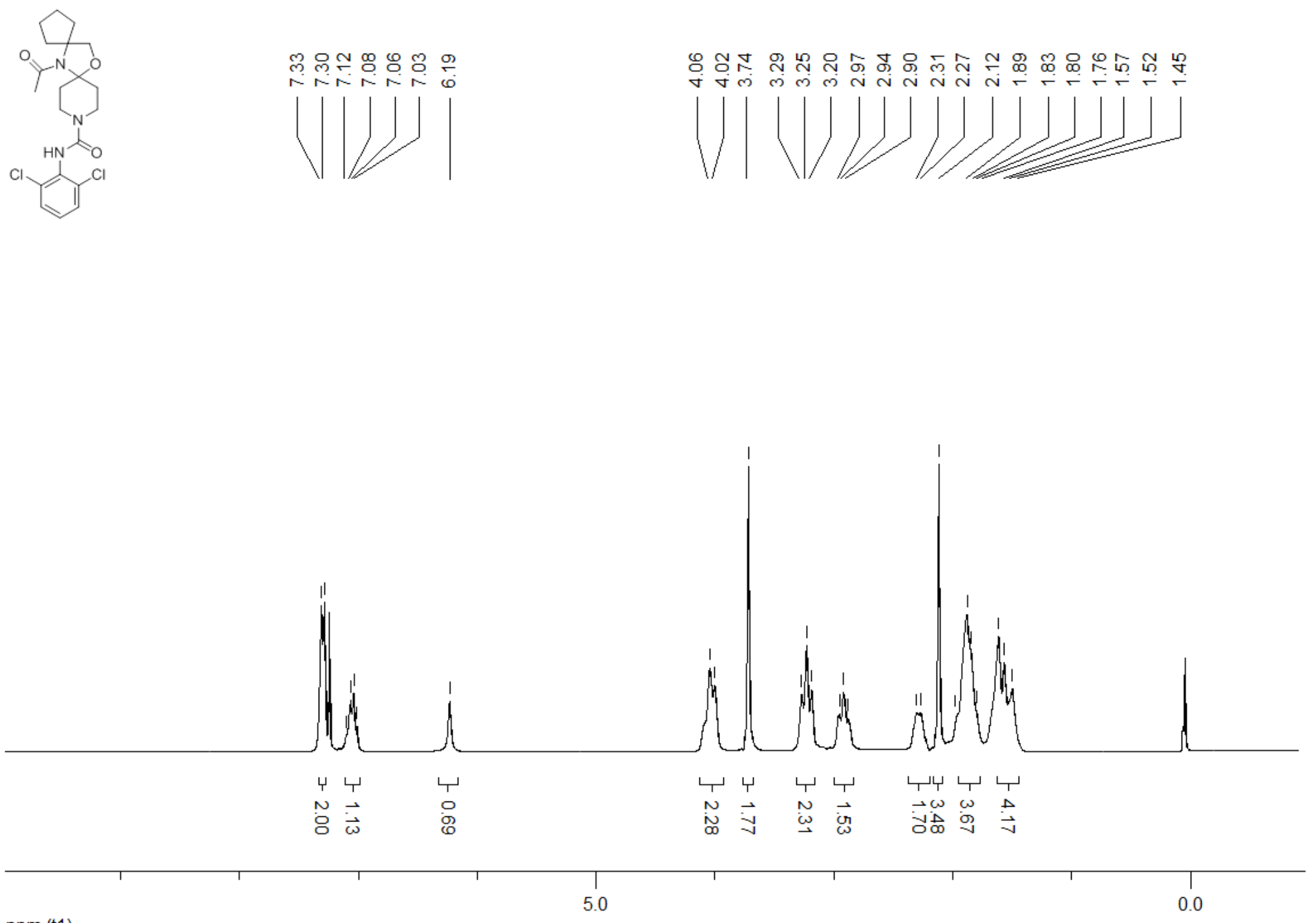

ppm (t1) 
${ }^{1}$ H NMR (300 MHz, CDCl $)$ 6-acetyl-N-[4-(trifluoromethoxy)phenyl]-13-oxa-6,10-diazadispiro[4.1.5 $5^{7} .^{5}$ ]tetradecane-10-carboxamide $22\{3.2\}$
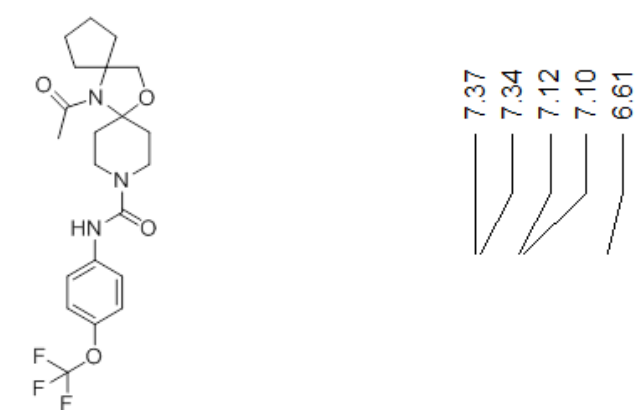

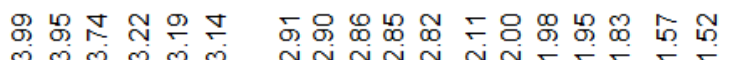
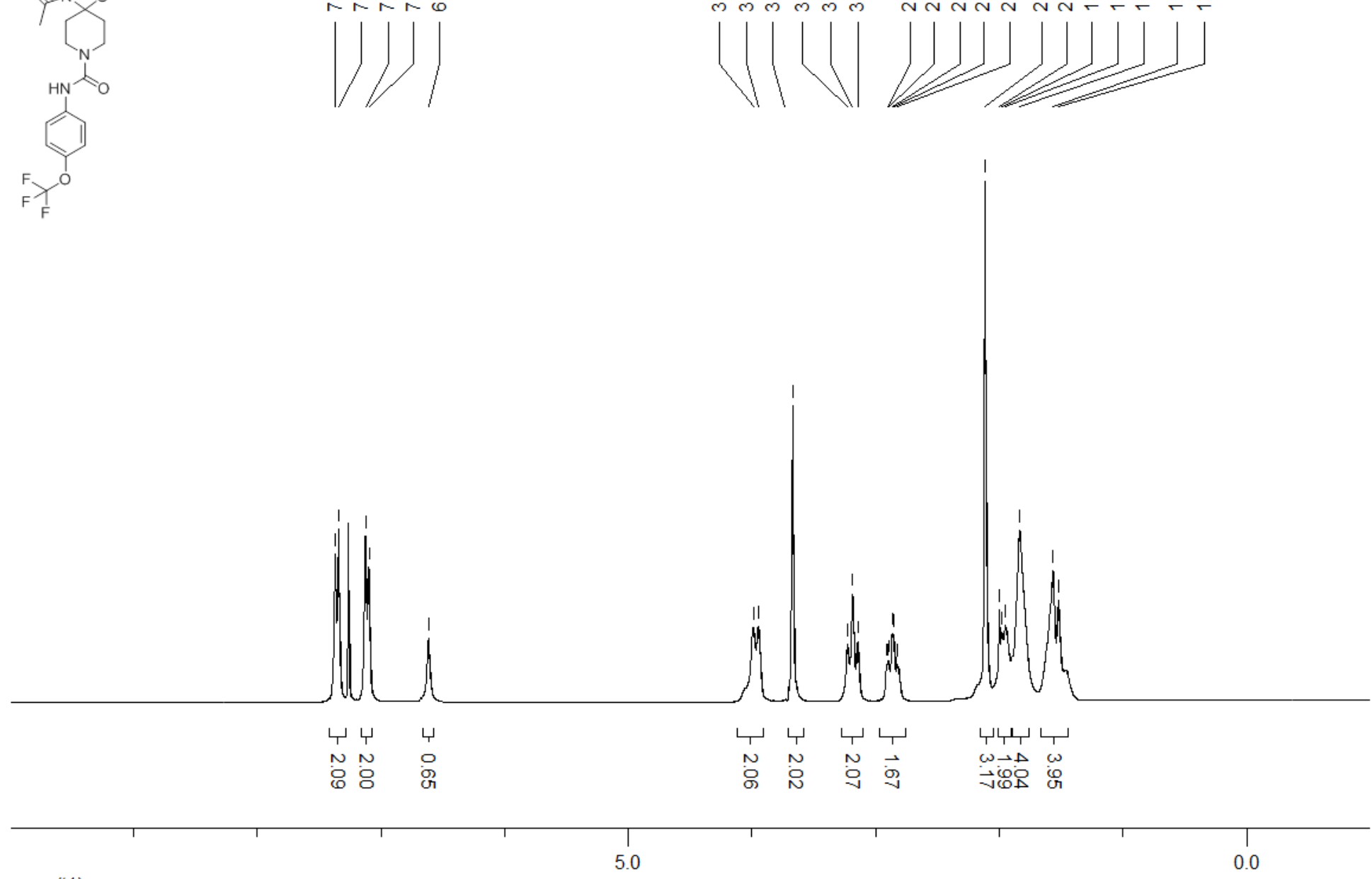

ppm (t1) 
HPLC-MS (ESI) ) 6-acetyl-N-[4-(trifluoromethoxy)phenyl]-13-oxa-6,10-diazadispiro[4.1.5.$\left.^{7} 2^{5}\right]$ tetradecane-10-carboxamide 22 $\{3.2\}$

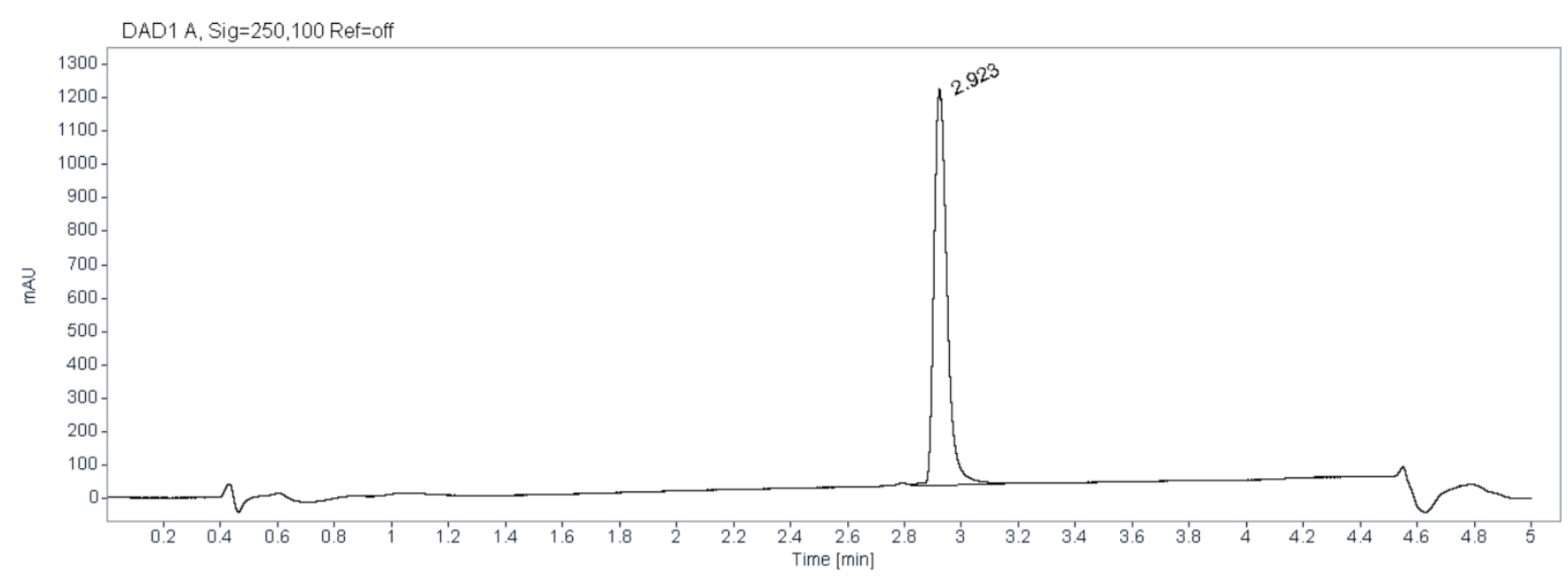

MSD1 SPC, MM-ES+APCI

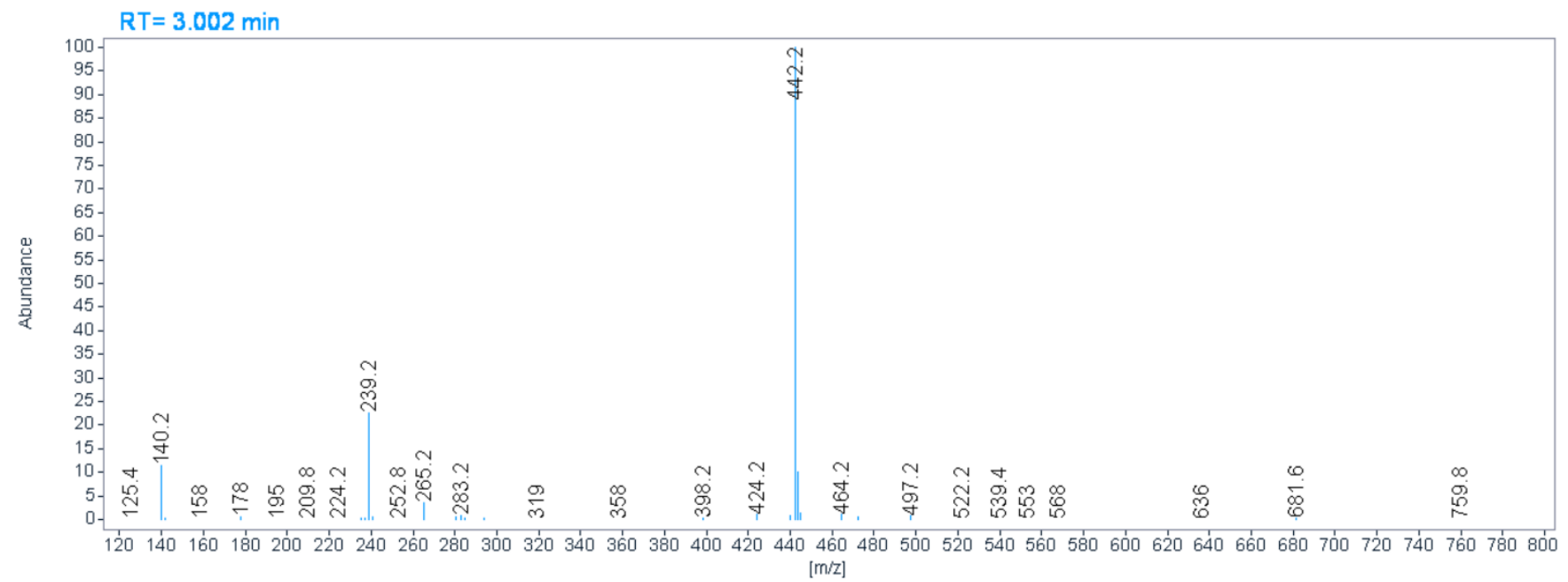




\section{References}

(1) Chaturvedula, P. V.; Pin, S.; Tholady, G.; Conway, C. M.; Macor, J. E.; Dubowchik, G. M. Design and Synthesis of Potent Antagonists Containing Rigid Spirocyclic Privileged Structures for the CGRP Receptor. Bioorg. Med. Chem. Lett. 2012, 22 (14), 4719-4722.

(2) Albert, J. S.; Aharony, D.; Andisik, D.; Barthlow, H.; Bernstein, P. R.; Bialecki, R. A.; Dedinas, R.; Dembofsky, B. T.; Hill, D.; Kirkland, K.; Koether, G. M.; Kosmider, B. J.; Ohnmacht, C.; Palmer, W.; Potts, W.; Rumsey, W.; Shen, L.; Shenvi, A.; Sherwood, S.; Warwick, P. J.; Russell, K. Design, Synthesis, and SAR of Tachykinin Antagonists: Modulation of Balance in NK1/NK2 Receptor Antagonist Activity. J. Med. Chem. 2002, 45 (18), 3972-3983.

(3) Ushakov, D. B.; Gilmore, K.; Kopetzki, D.; McQuade, D. T.; Seeberger, P. H. Continuous-Flow Oxidative Cyanation of Primary and Secondary Amines Using Singlet Oxygen. Angew. Chem. Int. Ed. 2014, 53 (2), 557-561.

(4) Altman, M.; Childers, K. K.; Donofrio, A.; Ellis, J. M.; Knowles, S. L.; Northrup, A. 3PYRIDYL CARBOXAMIDE-CONTAINING SPLEEN TYROSINE KINASE (Syk) INHIBITORS. WO2013052393 (A1), April 11, 2013.

(5) Bekkali, Y.; Betageri, R.; Emmanuel, M. J.; Hickey, E. R.; Liu, W.; Patel, U. R.; Spero, D. M.; Thomson, D. S.; Ward, Y. D.; Young, E. R. R.; Sun, S. Succinate Derivative Compounds Useful as Cysteine Protease Inhibitors. US6313117 (B1), November 6, 2001 .

(6) Kong, X.; Wu, X.; Bouzide, A.; Valade, I.; Migneault, D.; Gervais, F.; Delorme, D.; Bachand, B.; Atfani, M.; Levesque, S.; Samim, B. Methods and Compositions for Treating Amyloid-Related Diseases. US2006223855 (A1), October 5, 2006.

(7) Tsang, J. W.; Schmied, B.; Nyfeler, R.; Goodman, M. Peptide Sweeteners. 6. Structural Studies on the C-Terminal Amino Acid of L-Aspartyl Dipeptide Sweeteners. J. Med. Chem. 1984, 27 (12), 1663-1668.

(8) Palacin, S.; Chin, D. N.; Simanek, E. E.; MacDonald, J. C.; Whitesides, G. M.; McBride, M. T.; Palmore, G. T. R. Hydrogen-Bonded Tapes Based on Symmetrically Substituted Diketopiperazines: A Robust Structural Motif for the Engineering of Molecular Solids. J. Am. Chem. Soc. 1997, 119 (49), 11807-11816.

(9) Hayashi, S.; Nakata, E.; Morita, A.; Mizuno, K.; Yamamura, K.; Kato, A.; Ohashi, K. Discovery of $\{1-[4-(2-\{$ hexahydropyrrolo[3,4-C]pyrrol-2(1H)-Yl\}-1H-Benzimidazol-1Y1)piperidin-1-Y1]cyclooctyl\}methanol, Systemically Potent Novel Non-Peptide 
Agonist of Nociceptin/orphanin FQ Receptor as Analgesic for the Treatment of Neuropathic Pain: Design, Synthesis, and Structure-activity Relationships. Bioorg. Med. Chem. 2010, 18 (21), 7675-7699.

(10) Dixon, B. R.; Bagi, C. M.; Brennan, C. R.; Brittelli, D. R.; Bullock, W. H.; Chen, J.; Collibee, W. L.; Dally, R.; Johnson, J. S.; Kluender, H. C. E.; Lathrop, W. F.; Liu, P.; Mase, C. A.; Redman, A. M.; Scott, W. J.; Urbahns, K.; Wolanin, D. J. Substituted 2Arylimino Heterocycles and Compositions Containing Them, for Use as Progesterone Receptor Binding Agents. US6353006 (B1), March 5, 2002.

(11) Player, M.; Parks, D.; Parsons, W.; Meegalla, S.; Illig, C.; Ballentine, S. Heterocyclic Benzimidazoles as Trpm8 Modulators. WO2010045166 (A1), April 22, 2010. 